GEOLOGICAL SURVEY CIRCULAR 301

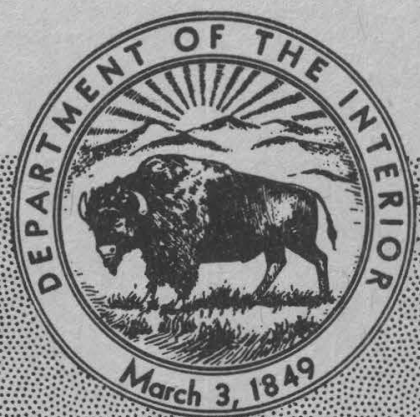

STRATIGRAPHIC SECTIONS OF THE PHOSPHORIA FORMATION IN

IDAHO, 1947-48, PART 2 



\author{
UNITED STATES DEPARTMENT OF THE INTERIOR \\ Douglas McKay, Secretary \\ GEOLOGICAL SURVEY \\ W. E. Wrather, Director
}

GEOLOGICAL SURVEY CIRCULAR 301

\title{
STRATIGRAPHIC SECTIONS OF THE PHOSPHORIA FORMATION IN IDAHO, 1947-48, PART 2
}

By V. E. McKelvey, F. C. Armstrong, R. A. Gulbrandsen, and R. M. Campbell

This report concerns work done partly on behalf.of the

U. S. Atomic Energy Commission

and is published with the permission of the Commission. 


\title{
STRATIGRAPHIC SECTIONS OF THE PHOSPHORIA FORMATION IN IDAHO, 1947-48, PART 2
}

\author{
By V. E. McKelvey, F. C. Armstrong; R. A. Gulbrandsen, and R. M. Campbell
}

CONTENTS

Lot no. Page

Lot no. Page

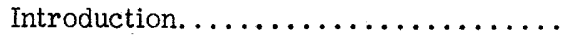

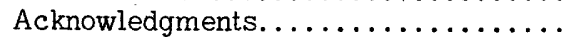
Stratigraphy of the Phosphoria

formation in southeastern Idaho..... . Stratigraphic sections............

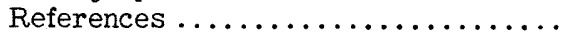
Tables of stratigraphic sections and chemical analyses

North Wooley Range ........... Spectrographic analyses....

North Rasmussen Valley ........ Spectrographic analyses....
1

1

1

4

4

$1230 \quad 5$

8
$1233 \quad 10$

14
Tables of stratigraphic sections and chemical analyses-Continued South Rasmussen Valley..... . . . $1232 \quad 16$ Spectrographic analyses..... Trail Canyon............... 1206 Spectrographic analyses..... Johnson Creek ................ 1209 Spectrographic analyses..... South Dry Valley ............. 1211 West Dairy . . . . . . . . . . . . 1208 Montpelier Canyon...... 1207 and 1236 Spectrographic analyses.....

\section{ILLUSTRATIONS}

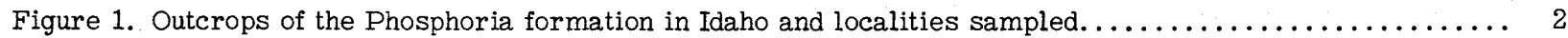

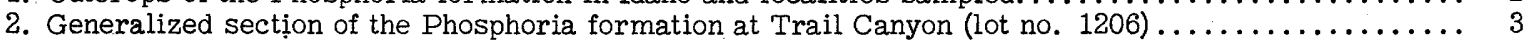

\section{INTRODUCTION}

The U. S. Geological Survey has measured and sampled the Phosphoria formation at many localities in Idaho and other western states. These data will not be fully synthesized and analyzed for several years, but segments of the data, accompanied by little or no interpretation, are published as preliminary reports as they are assembled. This report, which contains abstracts of many of the sections in southeastern Idaho (fig. 1), is one of this series and is the second report of data gathered in Idaho during 1947 and 1948 . The field and laboratory procedures adopted in these investigations are described rather fully in a companion report (McKelvey and others, 1953).

Many people have taken part in this investigation. In addition to the authors, D. F. Davidson, J. A. Noel, F. W. O'Malley, R. L. Parker, O. A. Payne, R. S. Sears, R. P. Sheldon, R. A. Smart, R. G. Waring, C. E. Weaver, $\mathrm{H}$. Wedow, and R. A. Weeks participated in the description of the strata and the collection of the samples referred to in this report. D. B. Dimick, JackGeorge, W. S. Hunziker, J. E. Jones, H. A. Larsen, and T. K. Rigby assisted in the preparation of trenches and the collection, crushing, and splitting of samples in the field. The laboratory preparation of samples for chemical analysis was done in Denver, Colo., under the direction of W. P. Huleatt.

All the $\mathrm{P}_{2} \mathrm{O}_{5}, \mathrm{~F}$, and acid-insoluble analyses were made for the Survey by the U. S. Bureau of Mines at the Northwest Electrodevelopment Laboratory, Albany, Oreg., under the direction of S. M. Shelton and M. L. Wright, and some of the spectrographic analyses were made there by D. M. Mortimer. The $\mathrm{Al}_{2} \mathrm{O}_{3}, \mathrm{Fe}_{2} \mathrm{O}_{3}$, and loss-on-ignition analyses were made in the Trace Elements Section laboratory of the Survey in Washington, D. C. , under the direc- tion of J. C. Rabbitt, by chemists I. Barlow, J. L. Greene, H. Levine, H. Mela, Jr., and A. L. White, and most of the spectrographic reports were prepared in this laboratory by C. Annell, C. L. Waring, and $\mathrm{H}$. Worthington.

Compilation of the data has been largely by R. P. Sheldon and F. D. Frieske under the supervision of R. W. Swanson. Organization of the tabular data has been largely by Anita Cozzetto.

\section{ACKNOWLEDGMENTS}

Special thanks are due W. W. Rubey, J. Steele Williams, and A. E. Weissenborn who have given much advice in planning and organizing the field program.

The cost of both the field and laboratory investigations has been partly borne by the Division of Raw Materials of the Atomic Energy Commission.

It is a pleasure to acknowledge the fine cooperation extended to the field party by the local residents, property owners, and operating phosphate companies, who furnished information and services and gave access to property. A. J. Winter, Superintendent of the Montpelier schools; E. M. Norris, C. T. Russell, and L. E. Traeger of the Anaconda Copper Mining Co.; D. L. King of the San Francisco Chemical Co. ; and G. A. McHugh and H. B. Fowler of the Simplot Fertilizer Co. have been especially helpful in this connection.

\section{STRATIGRAPHY OF THE PHOSPHORIA FORMATION IN SOUTHEASTERN IDAHO}

At its type locality in southeastern Idaho (Richards and Mansfield, 1912), the Phosphoria formation consists 


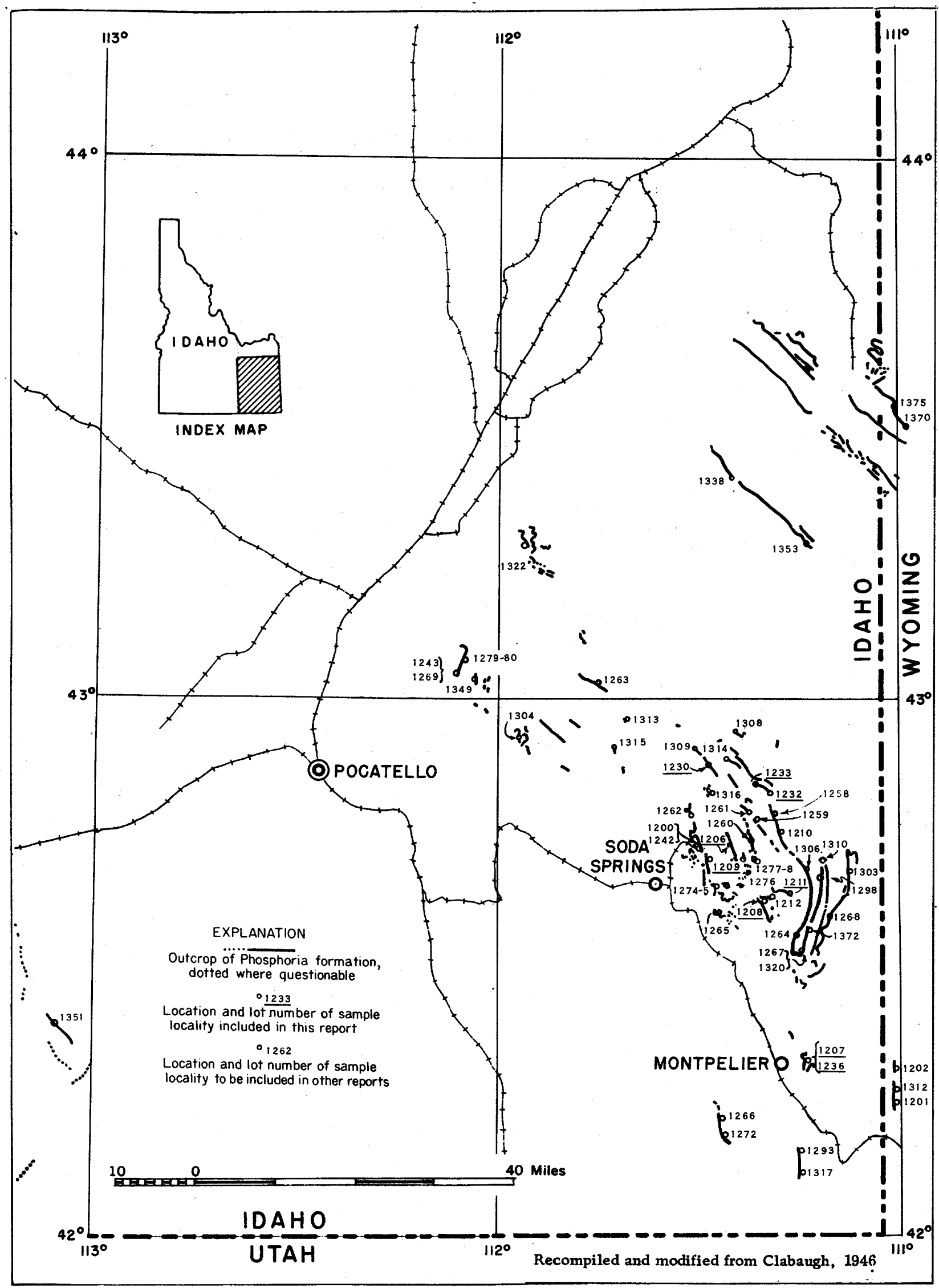

Figure 1. - Outcrops of the Phosphoria formation in Idaho and localities sampled. 


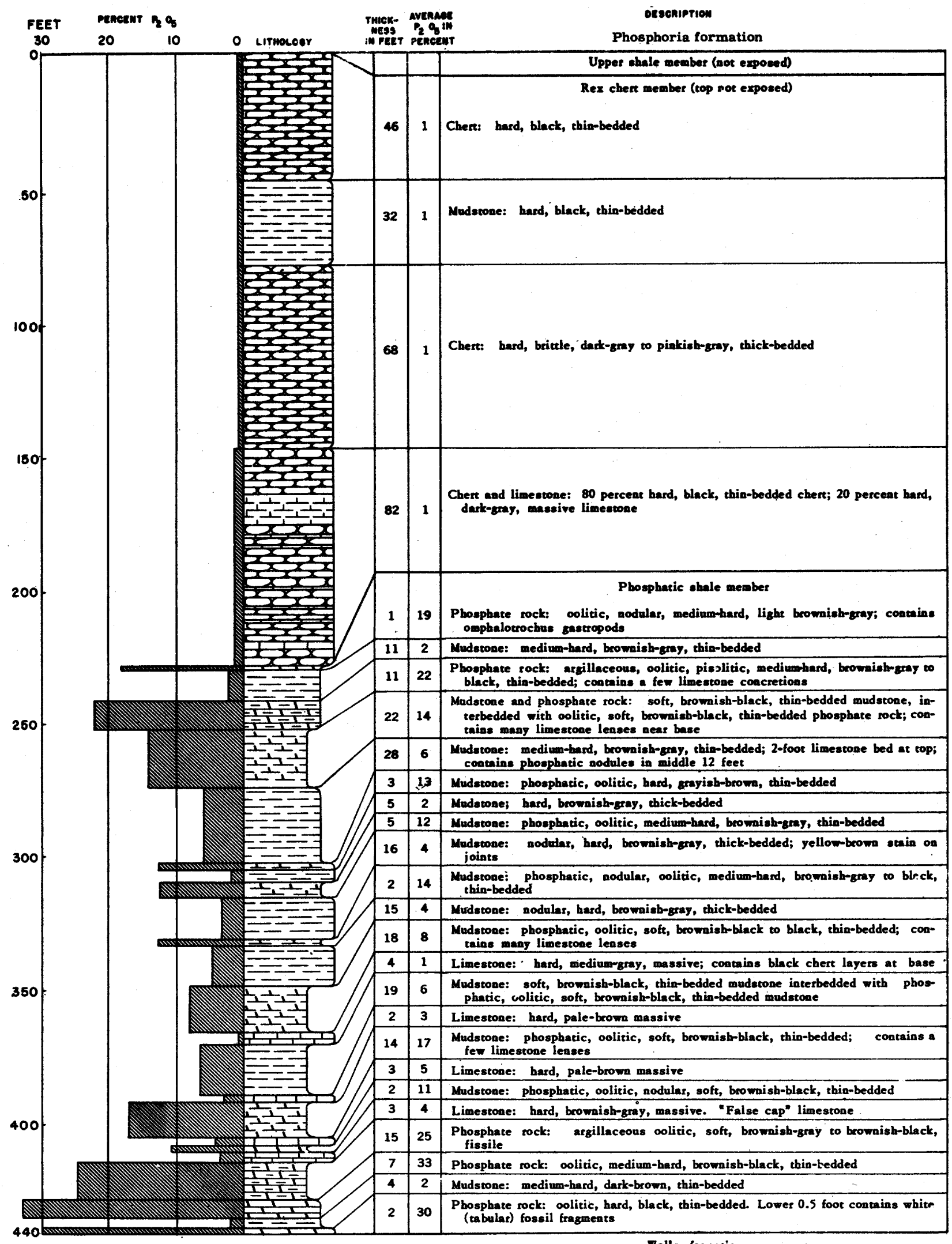


of a lower member, the phosphatic shale, about 180 feet thick, and an upper member, the Rex chert, about 240 feet thick; in most of southeastern Idaho and western Wyoming, however, another member, a thinbedded cherty mudstone 15 to 75 feet thick, overlies the Rex chert member, though it is not well defined at the type locality.

The Phosphoria formation overlies the Wells formation of Pennsylvanian age, and underlies the Dinwoody formation of Triassic age. The upper 50 to 75 feet of the Wells formation consists of gray fossiliferous cherty limestone that contains some thin phosphatic layers. It may be the correlative of the lowermost member (A member) of the Phosphoria formation in Montana and the lower limestone member of the Park City formation in Utah (McKelvey, 1949).

In southeastern Idaho most of the phosphatic beds are in the phosphatic shale member, and it is on this member that most of our studies have been focused. It. consists of many thin layers, some of which persist over the whole area. They may be grouped into several broad units, as yet unnamed, as shown on figure 2 .

\section{STRATIGRAPHIC SECTIONS}

Abstracts of stratigraphic sections measured at 8 localities, and the available analytical data, are presented in the following pages. Their locations, as well as the locations of the sections reported previously (McKelvey and others, 1953) and of others to be reported later, are shown on figure 1.

The semiquantitative analyses are based upon comparisons with a standard plate representing known quantities of the elements tested for and made at the same exposure. Greater sensitivities for many elements can be obtained by additional exposures. The standard sensitivities for the elements noted in this report are as follows:

\begin{tabular}{|c|c|c|c|c|c|c|c|c|c|}
\hline \multirow{2}{*}{ Element } & \multicolumn{4}{|c|}{$\begin{array}{l}\text { Sensitivity (percent) for } \\
\text { indicated lot }\end{array}$} & \multirow{2}{*}{ Element } & \multicolumn{4}{|c|}{$\begin{array}{l}\text { Sensitivity (percent) for } \\
\text { indicated lot }\end{array}$} \\
\hline & 1230 & $\begin{array}{c}1232 \text { and } \\
1233\end{array}$ & $\begin{array}{c}1206 \text { and } \\
1209\end{array}$ & $\begin{array}{c}1207 \text { and } \\
1236\end{array}$ & & 1230 & $\begin{array}{l}1232 \text { and } \\
1233\end{array}$ & $\begin{array}{c}1206 \text { and } \\
1209\end{array}$ & $\begin{array}{l}1207 \text { and } \\
1236\end{array}$ \\
\hline & 0.0001 & 0.0001 & 0.001 & 0.005 & & 0.1 & 0.1 & 0.1 & \\
\hline & .001 & .001 & .001 & .05 & Mo- & .001 & .001 & .001 & 0.004 \\
\hline As- & .1 & .1 & .1 & .1 & $\mathrm{Nd}-$ & .01 & .01 & .01 & --- \\
\hline $\mathrm{Ba}-\mathrm{-}$ & .001 & .0001 & .001 & .08 & $\mathrm{~N} 1-$ & .001 & .001 & .001 & .01 \\
\hline $\mathrm{Be}-1$ & .001 & .0001 & .001 & .001 & $\mathrm{Nb}--$ & .01 & .01 & .01 & .01 \\
\hline Bi-- & .001 & .001 & .001 & .002 & P-.- & .1 & .1 & .1 & .8 \\
\hline B--- & .001 & .001 & .001 & .001 & Pt-- & .01 & .01 & .01 & .01 \\
\hline $\mathrm{Cd}--$ & .01 & .01 & .01 & .1 & $\mathrm{~K}---$ & $* .1$ & *1.0 & .0001 & --- \\
\hline $\mathrm{Ca}--$ & .0001 & .001 & .0001 & .01 & Pr-- & .01 & .01 & --- & --- \\
\hline $\mathrm{Ce}---$ & .1 & .1 & .1 & -- & $\operatorname{Re}--$. & .1 & .1 & .1 & -- \\
\hline $\mathrm{Cs}--$ & 1.0 & 1.0 & 1.0 & --1 & $0-$ & 0.0 & 10.0 & 10.0 & --- \\
\hline Cr- - & .001 & .001 & .001 & .02 & Sm------- & .1 & .1 & --- & --- \\
\hline Co-- & .001 & .01 & .001 & .01 & $\mathrm{Sc}--$ & .1 & .1 & .1 & --- \\
\hline $\mathrm{Cu}---$ & .0001 & .0001 & .0001 & .001 & Si--- & .0001 & .0001 & .0001 & .002 \\
\hline Dy-- & .01 & .01 & --- & --- & $\mathrm{Ag}--$ & .001 & .001 & .001 & .001 \\
\hline Er--- & .01 & .01 & --- & --- & Na----- & *.1 & *.1 & .0001 & .05 \\
\hline Eu-- & .01 & .01 & --- & --- & Sr---- & .01 & .01 & .01 & .1 \\
\hline Gd---- & .01 & .01 & --- & --- & $\mathrm{Ta}-$ & .1 & .1 & .01 & 1.0 \\
\hline Ga---- & .01 & .01 & -- & $.05-$ & Te------ & .1 & .1 & -- & --- \\
\hline $\mathrm{Ge}---$ & .001 & .001 & .01 & .01 & Tb---- & .1 & .1 & -- & -- \\
\hline $\mathrm{Au}--.-$ & .01 & .01 & --- & .01 & Ti--.-. & .1 & .1 & 1.0 & --- \\
\hline Hf------ & .1 & .1 & --- & -- & Th----- & .1 & .1 & .1 & --- \\
\hline Ho----- & .01 & .01 & --- & -- & $\mathrm{Tm}-\cdots$ & .01 & .01 & --- & --- \\
\hline In-- & .001 & .001 & .001 & -- & $\mathrm{Sn}-\cdots$ & .01 & .01 & .01 & .01 \\
\hline Fen- & .001 & .001 & .001 & .005 & Ti------ & .001 & .001 & .001 & .002 \\
\hline La-- & .01 & .01 & .01 & --- & W-- - & .1 & .1 & .1 & .1 \\
\hline $\mathrm{Pb}----$ & .01 & .01 & .01 & .1 & V-D-D- & .01 & .01 & .01 & .01 \\
\hline Li--.- & & *.1 & --- & 1.2 & Yb- & .0001 & .0001 & -- & -- \\
\hline Lu----- & .01 & .01 & --- & --- & Y------ & .001 & .001 & .001 & --- \\
\hline Mg------ & .0001 & .0001 & .0001 & .001 & $\mathrm{Zn}-$ & .001 & .01 & .001 & .05 \\
\hline $\mathrm{Mn}-\cdots$ & .001 & .001 & .001 & .004 & Zr------- & .001 & .001 & .001 & .003 \\
\hline
\end{tabular}

* A greater sensitivity may be obtained by additional exposures.

\section{REFERENCES}

Clabaugh, P. S., 1946, Permian phosphate deposits of Montana, Idaho, Wyoming, and Utah: U. S. Geol. Survey, Strategic Minerals Investigations Preliminary Map 3-198.

McKelvey, V. E., 1949, Geological studies of the western phosphate field: Am. Inst. Min. Met. Eng. Mining Trans., v. 184, p. 270-279.
McKelvey, V. E., Davidson, D. F., O'Malley, F. W. and Smith, L. E., 1953, Stratigraphic sections of the Phosphoria formation in Idaho, 1947-48, part 1: U. S. Geol. Survey Circ. 208.

Richards, R. W., and Mansfield, G. R., 1912, The Bannock overthrust: a major fault in southeastern Idaho and northeastern Utah: Jour. Geology, v. 20, p. 684 . 
NORTH WOOLEY RANGE, IDAHO. LOT NO. 1230.

Phosphatic shale member of Phosphoria formation sampled in bulldozer trench on northeast limb of Wooley Valley anticline, sec. 24 , T. $6 \mathrm{~S}$., R. 42 E., Caribou County, Idaho. Beds strike N. $40^{\circ}$ W. and dip $57^{\circ}$ NE. Section measured by R. A. Hoppin, C. E. Weaver, R. L. Parker, R. P. Sheldon, F.W. ' Malley, and V. E. McKelvey and sampled by J. A. Noel and R. A. Smart in July 1948 . Samples analyzed for $P_{2} O_{5}$ and acid insoluble by U. S. Bureau of Mines Laboratory, Albany, Oregon, and for other constituents by Trace Elements Section Laboratory, U. ${ }^{5}$. Geological Survey, Washington, D. C.

\begin{tabular}{|c|c|c|c|c|c|c|c|c|c|c|}
\hline \multirow{2}{*}{$\begin{array}{c}\text { Bed } \\
\text { no. }\end{array}$} & \multirow[b]{2}{*}{ Rock description } & \multirow{2}{*}{$\begin{array}{c}\text { Sample } \\
\text { no. }\end{array}$} & \multirow{2}{*}{$\underset{\text { (feet) }}{\text { Thickness }}$} & \multicolumn{5}{|c|}{ Chemical analyses (percent) } & \multirow{2}{*}{$\begin{array}{l}\text { Cumulative } \\
\text { thickness } \\
\text { (feet) }\end{array}$} & \multirow{2}{*}{$\begin{array}{l}\text { Thickness } \mathbf{x} \\
\text { percent } \mathbf{P}_{2} \mathrm{O}_{5} \\
\text { (cumulative) }\end{array}$} \\
\hline & & & & $\mathrm{P}_{2} \mathrm{O}_{5}$ & $\mathrm{Al}_{2} \mathrm{O}_{3}$ & $\mathrm{Fe}_{2} \mathrm{O}_{3}$ & $\begin{array}{l}\text { Loss on } \\
\text { ignition }\end{array}$ & $\begin{array}{c}\text { Acid } \\
\text { insoluble }\end{array}$ & & \\
\hline \multicolumn{11}{|c|}{ Rex member of Phosphoria formation-basal bed only } \\
\hline $\mathbf{R}-\mathbf{1}$ & Chert & 1289- RAH & 7.4 & 1.1 & -- & -- & -- & 85.3 & 2.3 & 2.53 \\
\hline \multicolumn{11}{|c|}{ Phosphatic shale member of Phosphoria formation } \\
\hline $\begin{array}{l}\text { P-92 } \\
\text { P-91 } \\
\text { P-90 } \\
\text { P-89 } \\
\text { P-88 }\end{array}$ & $\begin{array}{l}\text { Mudstone } \\
\text { Mudstone } \\
\text { Mudstone, calcareous and mudstone } \\
\text { Mudstone } \\
\text { Mudstone; fos. col. no. } 48 \text {-JES- } 81^{1}\end{array}$ & $\begin{array}{l}\text { 1288- RAH } \\
1287-\text { RAH } \\
1286-\text { RAH } \\
1285-\text { RAH } \\
1284-\text { RAH }\end{array}$ & $\begin{array}{l}2.3 \\
1.7 \\
3.7 \\
3.3 \\
4.1\end{array}$ & $\begin{array}{l}2.8 \\
6.0 \\
0.2 \\
1.5 \\
3.5\end{array}$ & $\begin{array}{l}-- \\
-- \\
-- \\
--\end{array}$ & $\begin{array}{l}-- \\
-- \\
-- \\
--\end{array}$ & $\begin{array}{l}-- \\
-- \\
-- \\
--\end{array}$ & $\begin{array}{l}68.7 \\
77.0 \\
67.6 \\
79.6 \\
71.9\end{array}$ & $\begin{array}{r}2.3 \\
4.0 \\
7.7 \\
11.0 \\
15.1\end{array}$ & $\begin{array}{r}6.44 \\
16.64 \\
17.38 \\
22.33 \\
36.68\end{array}$ \\
\hline $\begin{array}{l}P-87 \\
P-86 \\
P-85\end{array}$ & $\begin{array}{l}\text { Phosphate rock, argillaceous; fos. col. } \\
\text { no. } 48-\mathrm{JES}-80 \\
\text { Mudstone; fos. col. no. } 48 \text {-JES- } 79 \\
\text { Mudstone, calcareous; fos. col. no. }\end{array}$ & $\begin{array}{l}\text { 1283- RAH } \\
1282-\text { RAH }\end{array}$ & $\begin{array}{l}0.8 \\
2.0\end{array}$ & $\begin{array}{r}25.5 \\
5.6\end{array}$ & $=-$ & -- & $\begin{array}{l}-- \\
--\end{array}$ & $\begin{array}{l}24.8 \\
78.5\end{array}$ & $\begin{array}{l}15.9 \\
17.9\end{array}$ & $\begin{array}{l}57.08 \\
68.28\end{array}$ \\
\hline $\begin{array}{l}\text { P-84 } \\
\text { P-83 }\end{array}$ & $\begin{array}{l}\text { 48-JES-78 } \\
\text { Mudstone; fos. col. no. 48-JES-77 } \\
\text { Phosphate rock }\end{array}$ & $\begin{array}{l}1281-\text { RAH } \\
1280-\text { RAH } \\
1205-\text { RLP }\end{array}$ & $\begin{array}{l}0.8 \\
1.7 \\
1.7\end{array}$ & $\begin{array}{r}0.5 \\
6.1 \\
35.0\end{array}$ & $\begin{array}{l}-- \\
- \\
1.4\end{array}$ & $\begin{array}{l}-- \\
-- \\
0.46\end{array}$ & $\begin{array}{l}-- \\
5.90\end{array}$ & $\begin{array}{r}63.8 \\
80.7 \\
4.7\end{array}$ & $\begin{array}{l}18.7 \\
20.4 \\
22.1\end{array}$ & $\begin{array}{r}68.68 \\
79.05 \\
138.55\end{array}$ \\
\hline $\begin{array}{l}\text { P-82 } \\
\text { P-81 } \\
\text { P-80 } \\
\text { P-79 }\end{array}$ & $\begin{array}{l}\text { Mudstone and limestone } \\
\text { Phosphate rock } \\
\text { Phosphate rock } \\
\text { Mudstone, calcareous; fos. col. no. }\end{array}$ & $\begin{array}{l}1204-\text { RLP } \\
1203-\text { RLP } \\
1202-\text { RLP }\end{array}$ & $\begin{array}{l}1.6 \\
1.5 \\
0.8\end{array}$ & $\begin{array}{r}5.0 \\
33.1 \\
32.2\end{array}$ & $\begin{array}{l}8.5 \\
1.6 \\
2.2\end{array}$ & $\begin{array}{l}2.59 \\
0.74 \\
0.85\end{array}$ & $\begin{array}{r}12.32 \\
4.74 \\
4.64\end{array}$ & $\begin{array}{r}57.9 \\
9.4 \\
11.6\end{array}$ & $\begin{array}{l}23.7 \\
25.2 \\
26.0\end{array}$ & $\begin{array}{l}146.55 \\
196.20 \\
221.96\end{array}$ \\
\hline$P-78$ & $\begin{array}{l}\text { 48-JES-76 } \\
\text { Phosphate rock }\end{array}$ & $\begin{array}{l}1201-\text { RLP } \\
1200-\text { RLP }\end{array}$ & $\begin{array}{l}1.0 \\
1.2\end{array}$ & $\begin{array}{r}7.5 \\
29.4\end{array}$ & $\begin{array}{l}6.6 \\
3.3\end{array}$ & $\begin{array}{l}2.46 \\
0.96\end{array}$ & $\begin{array}{r}10.64 \\
5.22\end{array}$ & $\begin{array}{l}55.3 \\
16.8\end{array}$ & $\begin{array}{l}27.0 \\
28.2\end{array}$ & $\begin{array}{l}229.46 \\
264.74\end{array}$ \\
\hline $\begin{array}{l}P-77 \\
P-76 \\
P-75\end{array}$ & $\begin{array}{l}\text { Phosphate rock } \\
\text { Phosphate rock; fos. col. no. } 48-\mathrm{JES}-75 \\
\text { Phosphate rock, argillaceous; fos, col. }\end{array}$ & $\begin{array}{l}1305-W O M \\
1304-W O M\end{array}$ & $\begin{array}{l}0.6 \\
4.7\end{array}$ & $\begin{array}{l}36.3 \\
31.5\end{array}$ & $\begin{array}{l}0.76 \\
2.7\end{array}$ & $\begin{array}{l}0.37 \\
0.60\end{array}$ & $\begin{array}{l}4.80 \\
7.86\end{array}$ & $\begin{array}{l}4.4 \\
9.7\end{array}$ & $\begin{array}{l}28.8 \\
33.5\end{array}$ & $\begin{array}{l}286.52 \\
434.57\end{array}$ \\
\hline \multirow[t]{2}{*}{$\begin{array}{l}\text { P-74 } \\
P-73\end{array}$} & $\begin{array}{l}\text { no. 48-JES- } 74 \\
\text { Phosphate rock and phosphatic mudstone } \\
\text { Mudstone, phosphatic, and argillaceous }\end{array}$ & $\begin{array}{l}\text { 1212-CEW } \\
1211-\mathrm{CEW}\end{array}$ & $\begin{array}{l}3.55 \\
1.5\end{array}$ & $\begin{array}{l}25.2 \\
26.5\end{array}$ & $\begin{array}{l}3.9 \\
4.1\end{array}$ & $\begin{array}{l}1.2 \\
1.3\end{array}$ & $\begin{array}{l}9.60 \\
9.02\end{array}$ & $\begin{array}{l}21.0 \\
24.0\end{array}$ & $\begin{array}{l}37.05 \\
38.55\end{array}$ & $\begin{array}{l}524.03 \\
563.78\end{array}$ \\
\hline & phosphate rock; fos. col, no. 48-JES-73 & 1210-CEW & 1.6 & 15.8 & 7.5 & 2.2 & 9.80 & 46.4 & 40.15 & 589.06 \\
\hline $\begin{array}{l}P-72 \\
P-71\end{array}$ & $\begin{array}{l}\text { Mudstone, phosphatic; fos. col. no. } \\
48-J E S-72 \\
\text { Limestone and mudstone }\end{array}$ & $\begin{array}{l}1229-\text { RPS } \\
1228-\text { RPS }\end{array}$ & $\begin{array}{l}1.2 \\
2.8\end{array}$ & $\begin{array}{l}8.3 \\
0.7\end{array}$ & -- & $=$ & -- & $\begin{array}{l}62.4 \\
33.1\end{array}$ & $\begin{array}{l}11.35 \\
44.15\end{array}$ & $\begin{array}{l}599.02 \\
600.98\end{array}$ \\
\hline
\end{tabular}

${ }^{1}$ Fossil collection made by J. E. Smedley, Paleontology and Stratigraphy Branch, U. S. Geological Survey. 


\begin{tabular}{|c|c|c|c|c|c|c|c|c|c|c|}
\hline \multirow{2}{*}{$\begin{array}{l}\text { Bed } \\
\text { no. }\end{array}$} & \multirow[b]{2}{*}{ Rock description } & \multirow{2}{*}{$\begin{array}{l}\text { Sample } \\
\text { no. }\end{array}$} & \multirow{2}{*}{$\begin{array}{c}\text { Thickness } \\
\text { (feet) }\end{array}$} & \multicolumn{5}{|c|}{ Chemical analyses (percent) } & \multirow{2}{*}{$\begin{array}{l}\text { Cumulative } \\
\text { thickness } \\
\text { (feet) }\end{array}$} & \multirow{2}{*}{$\begin{array}{l}\text { Thickness } x \\
\text { percent } \mathrm{P}_{2} \mathrm{O}_{5} \\
\text { (cumulative) }\end{array}$} \\
\hline & & & & $\mathbf{P}_{2} \mathbf{O}_{5}$ & $\mathrm{Al}_{2} \mathrm{O}_{3}$ & $\mathrm{Fe}_{2} \mathrm{O}_{3}$ & $\begin{array}{l}\text { Loss on } \\
\text { ignition }\end{array}$ & $\begin{array}{l}\text { Acid } \\
\text { insoluble }\end{array}$ & & \\
\hline $\begin{array}{l}P-70 \\
P-69 \\
P-68 \\
P-67 \\
P-66\end{array}$ & $\begin{array}{l}\text { Mudstone, phosphatic, calcareous; } \\
\text { fos. col. no. } 48 \text {-JES-71 } \\
\text { Mudstone; fos. col. no. 48-JES-70 } \\
\text { Mudstone, phosphatic } \\
\text { Mudstone, phosphatic } \\
\text { Mudstone }\end{array}$ & $\begin{array}{l}1227-\text { RPS } \\
1226-\text { RPS } \\
1225-\text { RPS } \\
1224-\text { RPS } \\
1223-\text { RPS }\end{array}$ & $\begin{array}{l}2.3 \\
1.2 \\
1.3 \\
2.4 \\
1.1\end{array}$ & $\begin{array}{r}12.8 \\
5.4 \\
11.8 \\
11.6 \\
5.5\end{array}$ & $\begin{array}{l}-- \\
-- \\
-- \\
--\end{array}$ & $\begin{array}{l}-- \\
-- \\
-- \\
--\end{array}$ & $\begin{array}{l}-- \\
-- \\
-- \\
--\end{array}$ & $\begin{array}{l}45.0 \\
64.4 \\
53.3 \\
48.5 \\
71.1\end{array}$ & $\begin{array}{l}46.45 \\
47.65 \\
48.95 \\
51.35 \\
52.45\end{array}$ & $\begin{array}{l}630.42 \\
636.90 \\
652.24 \\
680.08 \\
686.13\end{array}$ \\
\hline $\begin{array}{l}P-65 \\
P-64 \\
P-63 \\
P-62 \\
P-61\end{array}$ & $\begin{array}{l}\text { Mudstone, phosphatic; fos. col. no. } \\
\text { 48-JES-69 } \\
\text { Phosphate rock, argillaceous } \\
\text { Mudstone; fos. col. no. } 48-J E S-68 \\
\text { Mudstone } \\
\text { Mudstone; fos. col. no. 48-JES-67 }\end{array}$ & $\begin{array}{l}1222-\text { RPS } \\
1218-\text { CEW } \\
1303-\text { WOM } \\
1302-\text { WOM } \\
1301-\text { WOM }\end{array}$ & $\begin{array}{l}3.1 \\
1.5 \\
1.0 \\
1.6 \\
1.2\end{array}$ & $\begin{array}{r}12.3 \\
25.5 \\
6.1 \\
2.8 \\
5.8\end{array}$ & $\begin{array}{l}-- \\
-- \\
-- \\
--\end{array}$ & $\begin{array}{l}-- \\
-- \\
-- \\
--\end{array}$ & $\begin{array}{l}-- \\
-- \\
-- \\
--\end{array}$ & $\begin{array}{l}54.6 \\
25.8 \\
75.9 \\
81.4 \\
80.2\end{array}$ & $\begin{array}{l}55.55 \\
57.05 \\
58.05 \\
59.65 \\
60.85\end{array}$ & $\begin{array}{l}724.26 \\
762.51 \\
768.61 \\
773.09 \\
780.05\end{array}$ \\
\hline $\begin{array}{l}P-60 \\
P-59\end{array}$ & $\begin{array}{l}\text { Mudstone, phosphatic } \\
\text { Mudstone, phosphatic; fos, col. no. }\end{array}$ & 1300-WOM & 0.8 & 11.4 & -- & -- & $\therefore$ & 61.0 & 61.65 & 789.17 \\
\hline $\begin{array}{l}P-57 \\
P-58 \\
P-57\end{array}$ & $\begin{array}{l}\text { 48-JES- } 66 \\
\text { Mudstone } \\
\text { Mudstone, phosphatic; fos. col. no. }\end{array}$ & $\begin{array}{l}1232-\text { WOM } \\
1231-\text { WOM }\end{array}$ & $\begin{array}{l}4.8 \\
0.6\end{array}$ & $\begin{array}{r}12.1 \\
6.9\end{array}$ & -- & -- & -- & $\begin{array}{l}58.3 \\
68.9\end{array}$ & $\begin{array}{l}66.45 \\
67.05\end{array}$ & $\begin{array}{l}847.25 \\
851.39\end{array}$ \\
\hline $\mathbf{P}-56$ & $\begin{array}{l}48-\mathrm{JES}-65 \\
\text { Mudstone and phosphate rock }\end{array}$ & $\begin{array}{l}1230-\text { WOM } \\
1215-\mathrm{CEW}\end{array}$ & $\begin{array}{l}0.8 \\
1.9\end{array}$ & $\begin{array}{r}9.4 \\
12.6\end{array}$ & -- & -- & -- & $\begin{array}{l}65.7 \\
49.6\end{array}$ & $\begin{array}{l}67.85 \\
69.75\end{array}$ & $\begin{array}{l}858.91 \\
882.85\end{array}$ \\
\hline $\begin{array}{l}P-55 \\
P-54 \\
P-53 \\
P-52 \\
P-51\end{array}$ & $\begin{array}{l}\text { Mudstone } \\
\text { Mudstone and phosphatic mudstone } \\
\text { Mudstone } \\
\text { Mudstone and phosphatic mudstone } \\
\text { Mudstone and phosphate rock }\end{array}$ & $\begin{array}{l}1214-\text { CEW } \\
1221-\text { RPS } \\
1220-\text { RPS } \\
1239-\text { RPS } \\
1238-\text { RPS }\end{array}$ & $\begin{array}{l}2.8 \\
2.7 \\
0.9 \\
1.2 \\
1.1\end{array}$ & $\begin{array}{r}3.3 \\
4.5 \\
1.8 \\
5.6 \\
24.0\end{array}$ & $\begin{array}{l}-- \\
-- \\
-- \\
--\end{array}$ & $\begin{array}{l}-- \\
-- \\
-- \\
--\end{array}$ & $\begin{array}{l}-- \\
-- \\
-- \\
--\end{array}$ & $\begin{array}{l}81.0 \\
71.7 \\
79.9 \\
73.6 \\
26.1\end{array}$ & $\begin{array}{l}72.55 \\
75.25 \\
76.15 \\
77.35 \\
78.45\end{array}$ & $\begin{array}{l}892.09 \\
904.24 \\
905.86 \\
912.58 \\
938.98\end{array}$ \\
\hline $\begin{array}{l}P-50 \\
P-49 \\
P-48 \\
P-47 \\
P-46\end{array}$ & $\begin{array}{l}\text { Mudstone and argillaceous phosphate } \\
\text { rock; fos, col. no. } 48-\mathrm{JES}-64 \\
\text { Mudstone and phosphate rock } \\
\text { Mudstone and phosphate rock } \\
\text { Mudstone; fos. col. no. } 48-\mathrm{JES}-63 \\
\text { Mudstone }\end{array}$ & $\begin{array}{l}1237-\text { RPS } \\
1236-\text { RPS } \\
1235-\text { RPS } \\
1234-\text { RPS } \\
1269-\text { VEM }\end{array}$ & $\begin{array}{l}0.8 \\
1.2 \\
1.0 \\
3.0 \\
2.9\end{array}$ & $\begin{array}{r}12.5 \\
16.9 \\
17.4 \\
5.9 \\
4.9\end{array}$ & $\begin{array}{l}-- \\
-- \\
-- \\
-- \\
--\end{array}$ & $\begin{array}{l}-- \\
-- \\
-- \\
--\end{array}$ & $\begin{array}{l}-- \\
-- \\
-- \\
--\end{array}$ & $\begin{array}{l}56.7 \\
38.6 \\
37.2 \\
72.7 \\
65.9\end{array}$ & $\begin{array}{l}79.25 \\
80.45 \\
81.45 \\
84.45 \\
87.35\end{array}$ & $\begin{array}{r}948.98 \\
969.26 \\
986.66 \\
1.004 .36 \\
1,018.57\end{array}$ \\
\hline $\begin{array}{l}P-45 \\
P-44 \\
P-43 \\
P-42 \\
P-41\end{array}$ & $\begin{array}{l}\text { Phosphate rock } \\
\text { Mudstone, phosphatic } \\
\text { Mudstone, calcareous, phosphatic } \\
\text { Mudstone, phosphatic } \\
\text { Mudstone }\end{array}$ & $\begin{array}{l}1268-V E M \\
1267-V E M \\
1266-V E M \\
1265-\text { VEM } \\
1264-\text { VEM }\end{array}$ & $\begin{array}{l}2.9 \\
3.0 \\
1.4 \\
0.6 \\
0.6\end{array}$ & $\begin{array}{r}31.8 \\
10.0 \\
10.7 \\
14.7 \\
5.8\end{array}$ & $\begin{array}{l}-- \\
-- \\
-- \\
--\end{array}$ & $\begin{array}{l}-- \\
-- \\
-- \\
--\end{array}$ & $\begin{array}{l}-- \\
-- \\
-- \\
--\end{array}$ & $\begin{array}{l}10.5 \\
60.5 \\
43.4 \\
37.4 \\
68.8\end{array}$ & $\begin{array}{l}90.25 \\
93.25 \\
94.65 \\
95.25 \\
95.85\end{array}$ & $\begin{array}{l}1,110.79 \\
1,140.79 \\
1,155.77 \\
1,164.59 \\
1,168.07\end{array}$ \\
\hline $\begin{array}{l}P-40 \\
P-39 \\
P-38 \\
P=37 \\
P-36\end{array}$ & $\begin{array}{l}\text { Mudstone, phosphatic } \\
\text { Mudstone, phosphatic } \\
\text { Mudstone, phosphatic } \\
\text { Mudstone, phosphatic } \\
\text { Limestone; fos. col. no. } 48 \text {-JEs- } 62\end{array}$ & $\begin{array}{l}1263-\text { VEM } \\
1262-\text { VEM } \\
1261-\text { VEM } \\
1260-\text { VEM } \\
1279-\text { WOM }\end{array}$ & $\begin{array}{l}0.8 \\
0.5 \\
1.1 \\
2.1 \\
1.0\end{array}$ & $\begin{array}{r}12.0 \\
8.0 \\
12.1 \\
16.5 \\
2.2\end{array}$ & $\begin{array}{l}-- \\
-- \\
-- \\
-- \\
--\end{array}$ & $\begin{array}{l}-- \\
-- \\
-- \\
--\end{array}$ & $\begin{array}{l}-- \\
-- \\
-- \\
--\end{array}$ & $\begin{array}{l}48.9 \\
59.9 \\
41.3 \\
43.5 \\
13.7\end{array}$ & $\begin{array}{r}96.65 \\
97.15 \\
98.25 \\
100.35 \\
101.35\end{array}$ & $\begin{array}{l}1,177.67 \\
1,181.67 \\
1,194.98 \\
1,229.63 \\
1,231.83\end{array}$ \\
\hline $\mathbf{P}-35$ & Limestone; fos. col. no. 48-JES-61 & 1278-WOM & 2.7 & 0.4 & -- & -- & -- & 9.7 & 104.05 & $1,232.91$ \\
\hline
\end{tabular}


Mudstone; fos. col. no. 48-JES-60

Mudstone, phosphatic

P-31 Mudstone, phosphatic; fos. col. no.

$$
\text { 48-JES-58 }
$$

P-30 Mudstone, phosphatic

P-29 Mudstone, phosphatic

P-28 Mudstone, phosphatic

P-27 Mudstone and phosphate rock

P-26 Mudstone and phosphate rock

P-25 Phosphate rock

P-24 Phosphate rock

P-23 Phosphate rock, argillaceous

P-22 Phosphate rock, argillaceous; fos. col. no. 48-JES-57

P-21 Mudstone, phosphatic

P-20 Mudstone and phosphate rock

P-19 Phosphate rock, argillaceous

P-18 Phosphate rock

P-17 Phosphate rock, argillaceous

P-16 Mudstone, phosphatic

P-15

P-14 Mudstone, phosphatic

P-13 Phosphate rock

P-12 Mudstone, phosphatic and phosphate rock

fos. col. no. 48-JES-56

$P-11$ Phosphate rock

P-10 Phosphate rock, argillaceous; fos. col. no. 48-JES-55

P- 9 Phosphate rock, argillaceous

P- 8 Phosphate rock

P- 7 Phosphate rock

P- 6 Mudstone

P- 5 Limestone, argillaceous

P- 4 Mudstone; fos. col. no. 48-JES-54

P- 3 Mudstone

P- 2 Limestone, argillaceous; fos. col. no.

P: 1 Phosphate rock

\begin{tabular}{|c|c|c|c|c|c|c|c|c|}
\hline $\begin{array}{l}1277-W O M \\
1276-W O M \\
1275-W O M\end{array}$ & $\begin{array}{l}1.0 \\
0.5 \\
1.8\end{array}$ & $\begin{array}{l}2.7 \\
8.9 \\
5.7\end{array}$ & $\begin{array}{l}-- \\
-- \\
--\end{array}$ & $\begin{array}{l}-- \\
-- \\
--\end{array}$ & $\begin{array}{l}-- \\
-- \\
--\end{array}$ & $\begin{array}{l}80.7 \\
56.5 \\
74.9\end{array}$ & $\begin{array}{l}105.05 \\
105.55 \\
107.35\end{array}$ & $\begin{array}{l}1,235.61 \\
1,240.06 \\
1,250.32\end{array}$ \\
\hline $\begin{array}{l}\text { 1274-WOM } \\
1273-W O M\end{array}$ & $\begin{array}{l}2.6 \\
3.5\end{array}$ & $\begin{array}{l}13.7 \\
14.5\end{array}$ & -- & $=$ & -- & $\begin{array}{l}52.7 \\
46.1\end{array}$ & $\begin{array}{l}109.95 \\
113.45\end{array}$ & $\begin{array}{l}1.285 .94 \\
1,336.69\end{array}$ \\
\hline $\begin{array}{l}1272-W O M \\
1247-\mathrm{CEW} \\
1246-\mathrm{CEW} \\
1245-\mathrm{CEW} \\
1244-\mathrm{CEW}\end{array}$ & $\begin{array}{l}1.0 \\
0.7 \\
3.2 \\
1.1 \\
3.3\end{array}$ & $\begin{array}{r}11.8 \\
7.9 \\
14.6 \\
16.3 \\
32.3\end{array}$ & $\begin{array}{l}-- \\
-- \\
-- \\
5.8 \\
2.1\end{array}$ & $\begin{array}{c}-- \\
-- \\
-- \\
2.44 \\
1.31\end{array}$ & $\begin{array}{l}-- \\
-- \\
-- \\
6.06 \\
5.40\end{array}$ & $\begin{array}{r}65.7 \\
65.7 \\
48.6 \\
36.7 \\
9.0\end{array}$ & $\begin{array}{l}114.45 \\
115.15 \\
118.35 \\
119.45 \\
122.75\end{array}$ & $\begin{array}{l}1,348.49 \\
1,354.02 \\
1,400.74 \\
1,418.67 \\
1,525.26\end{array}$ \\
\hline $\begin{array}{l}1243-C E W \\
1242-C E W\end{array}$ & $\begin{array}{l}2.2 \\
1.5\end{array}$ & $\begin{array}{l}28.5 \\
26.3\end{array}$ & $\begin{array}{l}3.6 \\
3.7\end{array}$ & $\begin{array}{l}1.97 \\
1.47\end{array}$ & $\begin{array}{l}5.12 \\
7.32\end{array}$ & $\begin{array}{l}15.5 \\
20.4\end{array}$ & $\begin{array}{l}124.95 \\
126.45\end{array}$ & $\begin{array}{l}1,587.96 \\
1,627.41\end{array}$ \\
\hline $\begin{array}{l}1241-C E W \\
1240-C E W \\
1309-C E W\end{array}$ & $\begin{array}{l}1.9 \\
2.3 \\
2.0\end{array}$ & $\begin{array}{l}25.6 \\
13.0 \\
16.8\end{array}$ & $\begin{array}{l}3.4 \\
6.5 \\
6.4\end{array}$ & $\begin{array}{l}1.29 \\
2.48 \\
2.02\end{array}$ & $\begin{array}{r}7.40 \\
11.54 \\
7.90\end{array}$ & $\begin{array}{l}21.9 \\
46.4 \\
41.6\end{array}$ & $\begin{array}{l}128.35 \\
130.65 \\
132.65\end{array}$ & $\begin{array}{l}1,676.05 \\
1,705.95 \\
1,739.55\end{array}$ \\
\hline $\begin{array}{l}1308-C E W \\
1307-C E W \\
1306-C E W \\
1219-C E W \\
1314-\text { RAH }\end{array}$ & $\begin{array}{l}1.2 \\
3.4 \\
4.0 \\
1.9 \\
2.5\end{array}$ & $\begin{array}{l}25.2 \\
33.8 \\
29.9 \\
16.3 \\
31.6\end{array}$ & $\begin{array}{l}4.8 \\
1.4 \\
2.1 \\
6.8 \\
1.9\end{array}$ & $\begin{array}{l}1.54 \\
0.71 \\
0.86 \\
2.45 \\
0.91\end{array}$ & $\begin{array}{l}4.64 \\
3.76 \\
6.32 \\
5.52 \\
4.56\end{array}$ & $\begin{array}{r}23.8 \\
6.1 \\
11.8 \\
48.3 \\
9.0\end{array}$ & $\begin{array}{l}133.85 \\
137.25 \\
141.25 \\
143.15 \\
145.65\end{array}$ & $\begin{array}{l}1,769.79 \\
1,884.71 \\
2,004.31 \\
2,035.28 \\
2,114.28\end{array}$ \\
\hline $\begin{array}{l}1313-\text { RAH } \\
1312-\text { RAH }\end{array}$ & $\begin{array}{l}1.6 \\
1.8\end{array}$ & $\begin{array}{r}9.7 \\
33.7\end{array}$ & $\begin{array}{l}9.4 \\
1.8\end{array}$ & $\begin{array}{l}3.10 \\
0.68\end{array}$ & $\begin{array}{l}3.80 \\
3.84\end{array}$ & $\begin{array}{r}62.2 \\
5.7\end{array}$ & $\begin{array}{l}147.25 \\
149.05\end{array}$ & $\begin{array}{l}2,129.80 \\
2,190.46\end{array}$ \\
\hline $\begin{array}{l}1311-\text { RAH } \\
1310-\text { RAH }\end{array}$ & $\begin{array}{l}1.5 \\
2.3\end{array}$ & $\begin{array}{l}24.6 \\
28.2\end{array}$ & $\begin{array}{l}5.5 \\
2.5\end{array}$ & $\begin{array}{l}1.57 \\
0.87\end{array}$ & $\begin{array}{l}5.32 \\
6.10\end{array}$ & $\begin{array}{l}23.5 \\
14.0\end{array}$ & $\begin{array}{l}150.55 \\
152.85\end{array}$ & $\begin{array}{l}2,227.36 \\
2,292.22\end{array}$ \\
\hline $1294-W O M$ & 0.6 & 22.9 & 0.76 & 0.55 & 4.16 & 28.7 & 153.45 & $2,305.96$ \\
\hline $\begin{array}{l}1299-W O M \\
1298-W O M \\
1297-W O M \\
1292-W O M \\
1291-W O M\end{array}$ & $\begin{array}{l}0.3 \\
2.9 \\
2.0 \\
1.2 \\
1.2\end{array}$ & $\begin{array}{r}27.0 \\
34.4 \\
29.9 \\
1.8 \\
0.3\end{array}$ & $\begin{array}{l}4.5 \\
0.94 \\
2.3 \\
-. \\
-.\end{array}$ & $\begin{array}{l}1.66 \\
0.47 \\
1.00 \\
-- \\
--\end{array}$ & $\begin{array}{l}5.36 \\
5.94 \\
7.56 \\
-- \\
--\end{array}$ & $\begin{array}{r}21.3 \\
3.4 \\
12.3 \\
72.6 \\
35.4\end{array}$ & $\begin{array}{l}153.75 \\
156.65 \\
158.65 \\
159.85 \\
161.05\end{array}$ & $\begin{array}{l}2,314.06 \\
2,413.82 \\
2,473.62 \\
2,475.79 \\
3,476.14\end{array}$ \\
\hline $\begin{array}{l}1290-W O M \\
1271-W O M\end{array}$ & $\begin{array}{l}0.4 \\
0.6\end{array}$ & $\begin{array}{l}0.9 \\
0.1\end{array}$ & -- & -- & -- & $\begin{array}{l}76.4 \\
79.4\end{array}$ & $\begin{array}{l}161.45 \\
162.05\end{array}$ & $\begin{array}{l}2,476.50 \\
2,476.56\end{array}$ \\
\hline $\begin{array}{l}1270-W O M \\
1296-W O M\end{array}$ & $\begin{array}{l}0.8 \\
0.4\end{array}$ & $\begin{array}{r}1.8 \\
32.7\end{array}$ & -- & -- & $=-$ & $\begin{array}{r}43.8 \\
5.9\end{array}$ & $\begin{array}{l}162.85 \\
163.25\end{array}$ & $\begin{array}{l}2.478 .00 \\
2.491 .08\end{array}$ \\
\hline
\end{tabular}

Wells formation

Cw-1 Limestone; fos. col. no. 48-JES-52

1295-WOM

\begin{tabular}{l|l|}
0.7 & 0.5 \\
\hline
\end{tabular}

-.


Semi-quantitative analyses of selected samples of the phosphatic shale member of the Phosphoria formation, North Wooley Range, Idaho (see immediately preceding pages for location of section, thickness and description of strata, and chemical analyses of samples). made by the U. S. Geological Survey, Geochemistry and Petrology Branch, Washington, D. C. In addition to the elements listed in the table below, Sb, As, Be, Bi, Cs, Co, Cb, Dy, Er, Eu, Gd, Au, Hf, Ho, Li, Lu, Nd, Pt, K, Pr, Rb, Sm, Sc, Ta, Te, Tb, Tl, Th, Tm, $\mathrm{Sn}, \mathrm{Ti}$, and $\mathrm{W}$ were looked for in all samples but were not detected.

\section{Explanation of symbols}

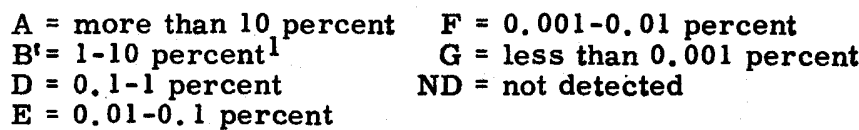

$F=0.001-0.01$ percent

$G=$ less than 0.001 percent $\mathrm{ND}=$ not detected

(

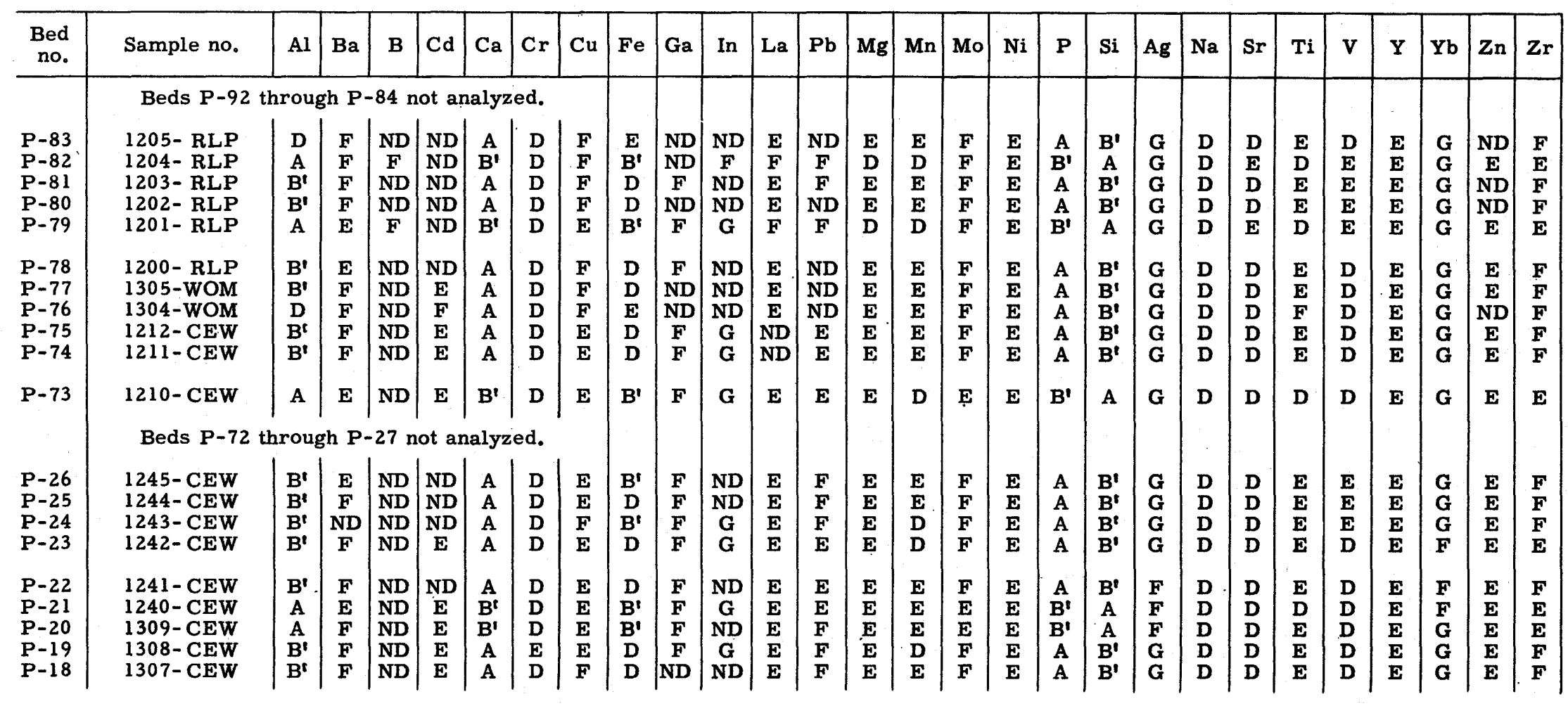




\begin{tabular}{|c|c|c|c|c|c|c|c|c|c|c|c|c|c|c|c|c|c|c|c|c|c|c|c|c|c|c|c|c|}
\hline $\begin{array}{l}P-17 \\
P-16 \\
P-15 \\
P-14 \\
P-13\end{array}$ & $\begin{array}{l}1306-\text { CEW } \\
1219-\mathrm{CEW} \\
1314-\mathrm{RAH} \\
1313-\mathrm{RAH} \\
1312-\mathrm{RAH}\end{array}$ & $\begin{array}{l}\mathbf{B}^{\prime} \\
\mathbf{A} \\
\mathbf{B}^{\prime} \\
\mathbf{B}^{\mathbf{t}} \\
\mathbf{B}^{\prime}\end{array}$ & $\begin{array}{l}\mathbf{F} \\
\mathbf{E} \\
\mathbf{F} \\
\mathbf{F} \\
\mathbf{F}\end{array}$ & $\begin{array}{l}\text { ND } \\
\text { ND } \\
\text { ND } \\
\text { ND } \\
\text { ND }\end{array}$ & $\begin{array}{c}E \\
N D \\
E \\
E \\
E\end{array}$ & $\begin{array}{l}\text { A } \\
\mathbf{B}^{\prime} \\
\mathbf{A} \\
\mathbf{A} \\
\mathbf{A}\end{array}$ & $\begin{array}{l}\mathbf{D} \\
\mathrm{D} \\
\mathbf{D} \\
\mathrm{D} \\
\mathrm{D}\end{array}$ & $\begin{array}{l}\mathbf{E} \\
\mathbf{E} \\
\mathbf{F} \\
\mathbf{F} \\
\mathbf{F}\end{array}$ & $\begin{array}{l}D \\
B^{\prime} \\
D \\
D \\
D\end{array}$ & $\begin{array}{c}\text { ND } \\
\mathbf{F} \\
\mathbf{F} \\
\mathbf{F} \\
\mathbf{F}\end{array}$ & $\begin{array}{l}\text { ND } \\
\text { G } \\
\text { ND } \\
\text { ND } \\
\text { ND }\end{array}$ & $\begin{array}{l}\mathbf{E} \\
\mathbf{E} \\
\mathbf{F} \\
\mathbf{F} \\
\mathbf{F}\end{array}$ & $\begin{array}{c}F \\
E \\
N D \\
N D \\
F\end{array}$ & $\begin{array}{l}\mathbf{E} \\
\mathbf{E} \\
\mathbf{E} \\
\mathbf{E} \\
\mathbf{E}\end{array}$ & $\begin{array}{l}\mathbf{E} \\
\mathbf{E} \\
\mathbf{E} \\
\mathbf{E} \\
\mathbf{E}\end{array}$ & $\begin{array}{l}\mathbf{F} \\
\mathbf{E} \\
\mathbf{F} \\
\mathbf{F} \\
\mathbf{F}\end{array}$ & $\begin{array}{l}\mathbf{E} \\
\mathbf{E} \\
\mathbf{E} \\
\mathbf{E} \\
\mathbf{E}\end{array}$ & $\begin{array}{l}\mathbf{A} \\
\mathbf{B}^{\mathfrak{l}} \\
\mathbf{A} \\
\mathbf{A} \\
\mathbf{A}\end{array}$ & $\begin{array}{l}\mathbf{B}^{\prime} \\
\mathbf{A} \\
\mathbf{B}^{\prime} \\
\mathbf{B}^{\prime} \\
\mathbf{B}^{\prime}\end{array}$ & $\begin{array}{l}\mathbf{G} \\
\mathbf{G} \\
\mathbf{G} \\
\mathbf{G} \\
\mathbf{G}\end{array}$ & $\begin{array}{l}\mathbf{D} \\
\mathbf{D} \\
\mathbf{D} \\
\mathbf{D} \\
\mathbf{D}\end{array}$ & $\begin{array}{l}\mathrm{D} \\
\mathbf{D} \\
\mathrm{D} \\
\mathrm{D} \\
\mathrm{D}\end{array}$ & $\begin{array}{l}\mathbf{E} \\
\mathbf{D} \\
\mathbf{E} \\
\mathbf{E} \\
\mathbf{E}\end{array}$ & $\begin{array}{l}\mathbf{D} \\
\mathbf{D} \\
\mathbf{D} \\
\mathbf{D} \\
\mathbf{D}\end{array}$ & $\begin{array}{l}\mathbf{E} \\
\mathbf{E} \\
\mathbf{E} \\
\mathbf{E} \\
\mathbf{E}\end{array}$ & $\begin{array}{l}\mathbf{G} \\
\mathbf{G} \\
\mathbf{G} \\
\mathbf{G} \\
\mathbf{G}\end{array}$ & $\begin{array}{l}\mathbf{E} \\
\mathbf{E} \\
\mathbf{E} \\
\mathbf{E} \\
\mathbf{E}\end{array}$ & $\begin{array}{l}\mathbf{F} \\
\mathbf{E} \\
\mathbf{F} \\
\mathbf{F} \\
\mathbf{F}\end{array}$ \\
\hline $\begin{array}{l}P-12 \\
P-11 \\
P-10 \\
P-9 \\
P-8\end{array}$ & $\begin{array}{l}1311-\text { RAH } \\
1310-\text { RAH } \\
1294-\text { WOM } \\
1299-\text { WOM } \\
1298-\text { WOM }\end{array}$ & $\begin{array}{l}B^{t} \\
B^{\prime} \\
B^{\prime} \\
B^{\prime} \\
D\end{array}$ & $\begin{array}{l}\mathbf{E} \\
\mathbf{F} \\
\mathbf{F} \\
\mathbf{F} \\
\mathbf{F}\end{array}$ & $\begin{array}{l}\text { ND } \\
\text { ND } \\
\text { ND } \\
\text { ND } \\
\text { ND }\end{array}$ & $\begin{array}{l}\mathbf{E} \\
\mathbf{E} \\
\mathbf{F} \\
\mathbf{F} \\
\mathbf{E}\end{array}$ & $\begin{array}{l}\mathbf{A} \\
\mathbf{A} \\
\mathbf{A} \\
\mathbf{A} \\
\mathbf{A}\end{array}$ & $\begin{array}{l}\mathbf{D} \\
\mathbf{D} \\
\mathbf{D} \\
\mathbf{D} \\
\mathbf{D}\end{array}$ & $\begin{array}{l}\mathbf{E} \\
\mathbf{E} \\
\mathbf{F} \\
\mathbf{F} \\
\mathbf{F}\end{array}$ & $\begin{array}{l}\mathbf{D} \\
\mathbf{D} \\
\mathbf{D} \\
\mathbf{D} \\
\mathbf{E}\end{array}$ & $\begin{array}{c}F \\
F \\
F \\
\text { ND } \\
\text { ND }\end{array}$ & $\begin{array}{c}\mathbf{G} \\
\mathbf{N D} \\
\mathbf{F} \\
\mathbf{G} \\
\mathbf{N D}\end{array}$ & $\begin{array}{l}\mathbf{E} \\
\mathbf{E} \\
\mathbf{E} \\
\mathbf{E} \\
\mathbf{E}\end{array}$ & $\begin{array}{l}\mathbf{E} \\
\mathbf{E} \\
\mathbf{E} \\
\mathbf{F} \\
\mathbf{F}\end{array}$ & $\begin{array}{l}\mathbf{E} \\
\mathbf{E} \\
\mathbf{E} \\
\mathbf{E} \\
\mathbf{E}\end{array}$ & $\begin{array}{l}\mathbf{D} \\
\mathbf{E} \\
\mathbf{D} \\
\mathbf{D} \\
\mathbf{E}\end{array}$ & $\begin{array}{c}\mathbf{F} \\
\mathbf{F} \\
\mathbf{F} \\
\mathbf{F} \\
\mathbf{N D}\end{array}$ & $\begin{array}{l}\mathbf{E} \\
\mathbf{E} \\
\mathbf{E} \\
\mathbf{E} \\
\mathbf{E}\end{array}$ & $\begin{array}{l}\mathbf{A} \\
\mathbf{A} \\
\mathbf{A} \\
\mathbf{A} \\
\mathbf{A}\end{array}$ & $\begin{array}{l}\mathbf{B}^{\prime} \\
\mathbf{B}^{\prime} \\
\mathbf{B}^{\prime} \\
\mathbf{B}^{\prime} \\
\mathbf{B}^{\prime}\end{array}$ & $\begin{array}{l}\mathbf{G} \\
\mathbf{G} \\
\mathbf{G} \\
\mathbf{G} \\
\mathbf{G}\end{array}$ & $\begin{array}{l}\mathbf{D} \\
\mathbf{D} \\
\mathbf{D} \\
\mathbf{D} \\
\mathbf{D}\end{array}$ & $\begin{array}{l}\mathbf{D} \\
\mathbf{D} \\
\mathbf{D} \\
\mathbf{D} \\
\mathbf{D}\end{array}$ & $\begin{array}{l}\mathbf{E} \\
\mathbf{E} \\
\mathbf{E} \\
\mathbf{E} \\
\mathbf{E}\end{array}$ & $\begin{array}{l}\mathbf{D} \\
\mathbf{D} \\
\mathbf{D} \\
\mathbf{D} \\
\mathbf{D}\end{array}$ & $\begin{array}{l}\mathbf{E} \\
\mathbf{E} \\
\mathbf{E} \\
\mathbf{E} \\
\mathbf{E}\end{array}$ & $\begin{array}{l}\mathbf{G} \\
\mathbf{G} \\
\mathbf{G} \\
\mathbf{G} \\
\mathbf{G}\end{array}$ & $\begin{array}{l}\mathbf{E} \\
\mathbf{E} \\
\mathbf{E} \\
\mathbf{E} \\
\mathbf{E}\end{array}$ & $\begin{array}{l}\mathbf{F} \\
\mathbf{F} \\
\mathbf{E} \\
\mathbf{F} \\
\mathbf{F}\end{array}$ \\
\hline$P-7$ & $\begin{array}{r}\text { 1297-WOM } \\
\text { Beds P-6 }\end{array}$ & $\mathbf{B}^{\mathbf{t}}$ & $\mathbf{F}$ & ND & $\mathbf{E}$ & A & D & $\mathbf{E}$ & $\mathbf{D}$ & ND & ND & $\mathbf{E}$ & $\mathbf{E}$ & $\mathbf{E}$ & $\mathbf{E}$ & $\mathbf{F}$ & $\mathbf{F}$ & A & $\mathbf{B}^{\prime}$ & $\mathbf{G}$ & D & D & $\mathbf{E}$ & D & $\mathbf{E}$ & $\mathbf{G}$ & $\mathbf{E}$ & $\mathbf{F}$ \\
\hline
\end{tabular}

${ }^{1} B^{\prime}$ is equivalent to $B$ and $C$ of Bureau of Mines analyses as recorded in other reports. 
NORTH RASMUSSEN VALLEY, IDAHO. LOT NO. 1233.

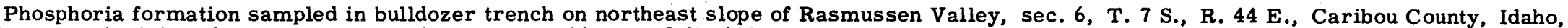

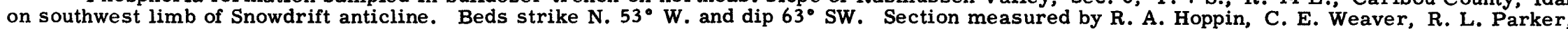

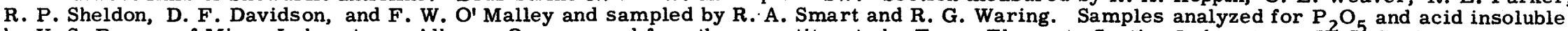

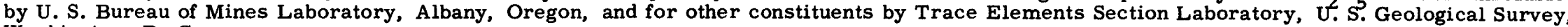
Washington, D. C.

\begin{tabular}{|c|c|c|c|c|c|c|c|c|c|c|}
\hline \multirow{2}{*}{$\begin{array}{c}\text { Bed } \\
\text { no. }\end{array}$} & \multirow[b]{2}{*}{ Rock description } & \multirow{2}{*}{$\begin{array}{c}\text { Sample } \\
\text { no. }\end{array}$} & \multirow{2}{*}{$\begin{array}{c}\text { Thickness } \\
\text { (feet) }\end{array}$} & \multicolumn{5}{|c|}{ Chemical analyses (percent) } & \multirow{2}{*}{$\begin{array}{l}\text { Cumulative } \\
\text { thickness } \\
\text { (feet) }\end{array}$} & \multirow{2}{*}{$\begin{array}{l}\text { Thickness } \mathrm{x} \\
\text { percent } \mathrm{P}_{2} \mathrm{O}_{5} \\
\text { (cumulative) }\end{array}$} \\
\hline & & & & $\mathrm{P}_{2} \mathrm{O}_{5}$ & $\mathrm{Al}_{2} \mathrm{O}_{3}$ & $\mathrm{Fe}_{2} \mathrm{O}_{3}$ & $\begin{array}{l}\text { Loss on } \\
\text { ignition }\end{array}$ & $\begin{array}{c}\text { Acid } \\
\text { insoluble }\end{array}$ & & \\
\hline \multicolumn{11}{|c|}{ Dinwoody formation - basal bed only } \\
\hline $\begin{array}{l}\text { Trd-2 } \\
\text { Trd-1 }\end{array}$ & $\begin{array}{l}\text { Siltstone } \\
\text { Phosphate rock, argillaceous, } \\
\text { conglomeratic }\end{array}$ & $\begin{array}{l}1418-\text { RAH } \\
1417-\text { RAH }\end{array}$ & $\begin{array}{l}4.0 \\
0.6\end{array}$ & $\begin{array}{r}0.5 \\
21.2\end{array}$ & -- & -- & -- & $\begin{array}{l}78.2 \\
31.6\end{array}$ & -- & -- \\
\hline \multicolumn{11}{|c|}{ Rex member of Phosphoria formation } \\
\hline $\begin{array}{l}R-24 \\
R-23 \\
R-22 \\
R-21 \\
R-20\end{array}$ & $\begin{array}{l}\text { Mudstone; fos. col. no. } 48-\mathrm{JES}-82^{1} \\
\text { Mudstone; fos. col. no. } 48-\mathrm{JES}-83 \\
\text { Mudstone } \\
\text { Phosphate rock, argillaceous } \\
\text { Mudstone }\end{array}$ & $\begin{array}{l}1416-\text { RAH } \\
1415-\text { RAH } \\
1414-\text { RAH } \\
1413-\text { RAH } \\
1412-\text { RAH }\end{array}$ & $\begin{array}{r}2.3 \\
10.0 \\
10.0 \\
2.0 \\
2.1\end{array}$ & $\begin{array}{r}4.0 \\
4.2 \\
5.6 \\
21.2 \\
2.2\end{array}$ & $\begin{array}{l}-- \\
-- \\
-- \\
--\end{array}$ & $\begin{array}{l}-- \\
-- \\
-- \\
-- \\
--\end{array}$ & $\begin{array}{l}-- \\
-- \\
-- \\
-- \\
--\end{array}$ & $\begin{array}{l}77.0 \\
71.6 \\
62.7 \\
31.4 \\
69.7\end{array}$ & $\begin{array}{l}2.3 \\
12.3 \\
22.3 \\
24.3 \\
26.4\end{array}$ & $\begin{array}{r}9.20 \\
51.20 \\
107.20 \\
149.60 \\
154.22\end{array}$ \\
\hline $\begin{array}{l}R-19 \\
R-18 \\
R-17 \\
R-16 \\
R-15\end{array}$ & $\begin{array}{l}\text { Mudstone } \\
\text { Mudstone, cherty } \\
\text { Mudstone; fos. col. no. } 48 \text {-JES-84 } \\
\text { Chert } \\
\text { Mudstone, cherty; fos. col. no. } \\
\quad 48 \text {-JES-85 }\end{array}$ & $\begin{array}{l}1411-\text { RAH } \\
1410-\text { RAH } \\
1409-\text { RAH } \\
1408-\text { RAH } \\
1407-\text { RAH }\end{array}$ & $\begin{array}{r}10.5 \\
7.0 \\
10.0 \\
2.0 \\
12.0\end{array}$ & $\begin{array}{l}3.1 \\
1.5 \\
1.9 \\
2.1 \\
2.4\end{array}$ & $\begin{array}{l}-- \\
-- \\
-- \\
--\end{array}$ & $\begin{array}{l}-- \\
-- \\
-- \\
--\end{array}$ & $\begin{array}{l}-- \\
-- \\
--\end{array}$ & $\begin{array}{l}73.6 \\
83.7 \\
82.4 \\
88.4 \\
73.3\end{array}$ & $\begin{array}{l}36.9 \\
43.9 \\
53.9 \\
55.9 \\
67.9\end{array}$ & $\begin{array}{l}186.77 \\
197.27 \\
216.27 \\
220.47 \\
249.27\end{array}$ \\
\hline $\begin{array}{l}R-14 \\
R-13 \\
R-12 \\
R-11 \\
R-10\end{array}$ & $\begin{array}{l}\text { Mudstone } \\
\text { Mudstone } \\
\text { Mudstone } \\
\text { Mudstone } \\
\text { Mudstone }\end{array}$ & $\begin{array}{l}1406-\text { RAH } \\
1405-\text { RAH } \\
1404-\text { RAH } \\
1403-\text { RAH } \\
1402-\text { RAH }\end{array}$ & $\begin{array}{l}10.0 \\
10.0 \\
10.0 \\
10.0 \\
10.0\end{array}$ & $\begin{array}{l}2.1 \\
1.3 \\
1.5 \\
1.6 \\
1.9\end{array}$ & $\begin{array}{l}-- \\
-- \\
-- \\
-- \\
--\end{array}$ & $\begin{array}{l}-- \\
-- \\
-- \\
--\end{array}$ & $\begin{array}{l}-- \\
-- \\
-- \\
-- \\
--\end{array}$ & $\begin{array}{l}77.0 \\
80.4 \\
81.2 \\
82.2 \\
76.5\end{array}$ & $\begin{array}{r}77.9 \\
87.9 \\
97.9 \\
107.9 \\
117.9\end{array}$ & $\begin{array}{l}270.27 \\
283.27 \\
298.27 \\
314.27 \\
333.27\end{array}$ \\
\hline $\begin{array}{l}R-9 \\
R-8 \\
R-7 \\
R-6 \\
R-5\end{array}$ & $\begin{array}{l}\text { Mudstone } \\
\text { Mudstone } \\
\text { Mudstone } \\
\text { Mudstone } \\
\text { Chert }\end{array}$ & $\begin{array}{l}1401-\text { RAH } \\
1400-\text { RAH } \\
1399-\text { RAH } \\
1398-\text { RAH } \\
1397-\text { RAH }\end{array}$ & $\begin{array}{r}10.0 \\
10.0 \\
10.0 \\
10.0 \\
5.4\end{array}$ & $\begin{array}{l}1.6 \\
1.3 \\
1.9 \\
1.2 \\
0.6\end{array}$ & $\begin{array}{l}-- \\
-- \\
-- \\
-- \\
--\end{array}$ & $\begin{array}{l}-- \\
-- \\
-- \\
-- \\
--\end{array}$ & $\begin{array}{l}-- \\
-- \\
-- \\
--\end{array}$ & $\begin{array}{l}79.2 \\
83.5 \\
81.3 \\
83.7 \\
92.2\end{array}$ & $\begin{array}{l}127.9 \\
137.9 \\
147.9 \\
157.9 \\
163.3\end{array}$ & $\begin{array}{l}349.27 \\
362.27 \\
381.27 \\
393.27 \\
396.51\end{array}$ \\
\hline $\begin{array}{l}R-4 \\
R-3 \\
R-2 \\
R-1\end{array}$ & $\begin{array}{l}\text { Chert and mudstone } \\
\text { Chert } \\
\text { Chert } \\
\text { Chert }\end{array}$ & $\begin{array}{l}1396-\text { RAH } \\
1395-\text { RAH } \\
1394-\text { RAH } \\
1384-\text { DFD }\end{array}$ & $\begin{array}{r}5.1 \\
4.0 \\
10.0 \\
10.0\end{array}$ & $\begin{array}{l}0.9 \\
1.2 \\
2.9 \\
0.2\end{array}$ & $\begin{array}{l}-- \\
-- \\
--\end{array}$ & $\begin{array}{l}-- \\
-- \\
--\end{array}$ & $\begin{array}{l}-- \\
-- \\
--\end{array}$ & $\begin{array}{l}88.5 \\
90.2 \\
87.1 \\
88.9\end{array}$ & $\begin{array}{l}168.4 \\
172.4 \\
182.4 \\
192.4\end{array}$ & $\begin{array}{l}401.10 \\
405.90 \\
434.90 \\
436.90\end{array}$ \\
\hline
\end{tabular}


Phosphatic shale member of Phosphoria formation

\begin{tabular}{|c|c|c|c|c|c|c|c|c|c|c|}
\hline $\begin{array}{l}P-83 \\
P-82 \\
P-81 \\
P-80 \\
P-79\end{array}$ & $\begin{array}{l}\text { Mudstone } \\
\text { Mudstone } \\
\text { Mudstone } \\
\text { Mudstone, phosphatic } \\
\text { Mudstone, phosphatic; fos. col. no. } \\
\text { 48-JES-103 }\end{array}$ & $\begin{array}{l}1383-\text { DFD } \\
1382-\text { DFD } \\
1381-\text { DFD } \\
1380-\text { DFD } \\
1339-\text { DFD }\end{array}$ & $\begin{array}{l}1.6 \\
0.9 \\
2.5 \\
0.9 \\
0.7\end{array}$ & $\begin{array}{r}1.3 \\
2.5 \\
3.9 \\
9.5 \\
16.1\end{array}$ & $\begin{array}{l}-- \\
-- \\
-- \\
--\end{array}$ & $\begin{array}{l}-- \\
-- \\
-- \\
--\end{array}$ & $\begin{array}{l}-- \\
-- \\
-- \\
--\end{array}$ & $\begin{array}{l}82.6 \\
70.6 \\
69.3 \\
40.1 \\
48.2\end{array}$ & $\begin{array}{l}1.6 \\
2.5 \\
5.0 \\
5.9 \\
6.6\end{array}$ & $\begin{array}{r}2.08 \\
4.33 \\
14.08 \\
22.63 \\
33.90\end{array}$ \\
\hline $\begin{array}{l}P-78 \\
P-77 \\
P-76 \\
P-75 \\
P-74\end{array}$ & $\begin{array}{l}\text { Mudstone } \\
\text { Mudstone; fos. col. no. } 48-J E S-104 \\
\text { Mudstone } \\
\text { Phosphate rock } \\
\text { Phosphate.rock and mudstone }\end{array}$ & $\begin{array}{l}1338-\text { DFD } \\
1337-\text { DFD } \\
1336-\text { DFD } \\
1335-\text { RLP } \\
1334-\text { RLP }\end{array}$ & $\begin{array}{l}1.7 \\
2.1 \\
0.8 \\
1.3 \\
1.0\end{array}$ & $\begin{array}{r}3.2 \\
1.1 \\
3.7 \\
36.2 \\
26.4\end{array}$ & $\begin{array}{l}-- \\
-- \\
\cdots \\
3.1\end{array}$ & $\begin{array}{l}-- \\
-- \\
-- \\
0.36 \\
1.64\end{array}$ & $\begin{array}{l}-- \\
-- \\
-- \\
6.50 \\
7.90\end{array}$ & $\begin{array}{r}76.6 \\
80.1 \\
73.1 \\
3.7 \\
15.2\end{array}$ & $\begin{array}{l}8.3 \\
10.4 \\
11.2 \\
12.5 \\
13.5\end{array}$ & $\begin{array}{r}39.34 \\
41.65 \\
44.61 \\
91.67 \\
118.07\end{array}$ \\
\hline $\begin{array}{l}P-73 \\
P-72 \\
P-71 \\
P-70 \\
P-69\end{array}$ & $\begin{array}{l}\text { Mudstone } \\
\text { Phosphate rock } \\
\text { Phosphate rock, argillaceous } \\
\text { Phosphate rock } \\
\text { Phosphate rock }\end{array}$ & $\begin{array}{l}1525-\text { WOM } \\
1524-\text { WOM } \\
1332-\text { RLP } \\
1331-\text { RLP } \\
1330-\text { RLP }\end{array}$ & $\begin{array}{l}0.4 \\
2.0 \\
1.8 \\
1.2 \\
1.0\end{array}$ & $\begin{array}{l}7.3 \\
31.3 \\
23.9 \\
33.8 \\
35.5\end{array}$ & $\begin{array}{l}8.4 \\
1.9 \\
4.9 \\
1.6 \\
1.5\end{array}$ & $\begin{array}{l}3.89 \\
0.94 \\
1.65 \\
0.58 \\
0.52\end{array}$ & $\begin{array}{r}9.50 \\
27.28 \\
8.08 \\
6.96 \\
5.12\end{array}$ & $\begin{array}{r}65.1 \\
12.3 \\
27.5 \\
5.7 \\
3.3\end{array}$ & $\begin{array}{l}13.9 \\
15.9 \\
17.7 \\
18.9 \\
19.9\end{array}$ & $\begin{array}{l}120.99 \\
183.59 \\
226.61 \\
267.17 \\
302.67\end{array}$ \\
\hline $\begin{array}{l}P-68 \\
P-67 \\
P-66 \\
P-65 \\
P-64\end{array}$ & $\begin{array}{l}\text { Phosphate rock } \\
\text { Phosphate rock } \\
\text { Mudstone, phosphatic } \\
\text { Mudstone } \\
\text { Mudstone, phosphatic }\end{array}$ & $\begin{array}{l}1523-\text { WOM } \\
1522-\text { WOM } \\
1379-\text { WOM } \\
1349-\text { RPS } \\
1348-\text { RPS }\end{array}$ & $\begin{array}{l}1.3 \\
4.1 \\
2.4 \\
1.3 \\
0.8\end{array}$ & $\begin{array}{r}29.9 \\
27.4 \\
13.1 \\
6.0 \\
11.3\end{array}$ & $\begin{array}{l}2.8 \\
3.2 \\
8.3 \\
-- \\
--\end{array}$ & $\begin{array}{l}1.05 \\
1.14 \\
2.37 \\
-. \\
\ldots\end{array}$ & $\begin{array}{r}11.32 \\
9.80 \\
11.04 \\
-- \\
--\end{array}$ & $\begin{array}{l}11.3 \\
17.4 \\
50.9 \\
67.3 \\
51.7\end{array}$ & $\begin{array}{l}21.2 \\
25.3 \\
27.7 \\
29.0 \\
29.8\end{array}$ & $\begin{array}{l}341.54 \\
453.88 \\
485.32 \\
493.12 \\
502.16\end{array}$ \\
\hline $\begin{array}{l}P-63 \\
P-62 \\
P-61 \\
P-60 \\
P-59\end{array}$ & $\begin{array}{l}\text { Mudstone and argillaceous phosphate rock } \\
\text { Mudstone and phosphate rock; fos. col. } \\
\text { no. } 48-\mathrm{JES}-105 \\
\text { Phosphate rock, argillaceous } \\
\text { Mudstone } \\
\text { Phosphate rock and mudstone }\end{array}$ & $\begin{array}{l}1347-\text { RPS } \\
1521-\text { WOM } \\
1520-\text { WOM } \\
1345-\text { RPS } \\
1344-\text { RPS }\end{array}$ & $\begin{array}{l}1.8 \\
1.2 \\
0.8 \\
1.4 \\
2.3\end{array}$ & $\begin{array}{r}11.3 \\
9.0 \\
19.5 \\
7.6 \\
16.8\end{array}$ & $\begin{array}{l}-- \\
-- \\
-- \\
--\end{array}$ & $\begin{array}{l}-- \\
-- \\
-- \\
--\end{array}$ & $\begin{array}{l}-- \\
-- \\
-- \\
--\end{array}$ & $\begin{array}{l}49.3 \\
52.7 \\
28.7 \\
63.3 \\
42.5\end{array}$ & $\begin{array}{l}31.6 \\
32.8 \\
33.6 \\
35.0 \\
37.3\end{array}$ & $\begin{array}{l}522.50 \\
533.30 \\
548.90 \\
559.54 \\
598.18\end{array}$ \\
\hline $\begin{array}{l}P-58 \\
P-57 \\
P-56 \\
P-55 \\
P-54\end{array}$ & $\begin{array}{l}\text { Phosphate rock, argillaceous } \\
\text { Mudstone } \\
\text { Mudstone, phosphatic } \\
\text { Mudstone, phosphatic; fos. col. no. } \\
48 \text {-JES-106 } \\
\text { Mudstone }\end{array}$ & $\begin{array}{l}1343-\text { RPS } \\
1342-\text { RPS } \\
1529-\text { WOM } \\
1528-\text { WOM } \\
1340-\text { RPS }\end{array}$ & $\begin{array}{l}1.4 \\
1.1 \\
2.6 \\
3.0 \\
0.6\end{array}$ & $\begin{array}{r}25.4 \\
6.6 \\
14.0 \\
8.3 \\
7.7\end{array}$ & $\begin{array}{l}-- \\
-- \\
-- \\
--\end{array}$ & $\begin{array}{l}-- \\
-- \\
-- \\
--\end{array}$ & $\begin{array}{l}-- \\
-- \\
-- \\
--\end{array}$ & $\begin{array}{l}26.3 \\
73.4 \\
52.6 \\
68.0 \\
66.3\end{array}$ & $\begin{array}{l}38.7 \\
39.8 \\
42.4 \\
45.4 \\
46.0\end{array}$ & $\begin{array}{l}633.74 \\
641.00 \\
677.40 \\
702.30 \\
706.92\end{array}$ \\
\hline $\begin{array}{l}P-53 \\
P-52 \\
P=51 \\
P-50 \\
P-49\end{array}$ & $\begin{array}{l}\text { Mudstone } \\
\text { Phosphate rock, argillaceous } \\
\text { Mudstone } \\
\text { Mudstone } \\
\text { Mudstone and phosphate rock }\end{array}$ & $\begin{array}{l}1369-\text { RAH } \\
1368-\text { RAH } \\
1367-\text { RAH } \\
1366-\text { RAH } \\
1365-\text { RAH }\end{array}$ & $\begin{array}{l}1.0 \\
0.6 \\
0.7 \\
1.1 \\
3.5\end{array}$ & $\begin{array}{r}7.4 \\
26.3 \\
6.8 \\
5.9 \\
7.4\end{array}$ & $\begin{array}{l}-- \\
-- \\
-- \\
--\end{array}$ & $\begin{array}{l}-- \\
-- \\
-- \\
--\end{array}$ & $\begin{array}{l}-- \\
-- \\
-- \\
--\end{array}$ & $\begin{array}{l}66.6 \\
22.0 \\
62.9 \\
69.2 \\
63.1\end{array}$ & $\begin{array}{l}47.0 \\
47.6 \\
48.3 \\
49.4 \\
52.9\end{array}$ & $\begin{array}{l}714.32 \\
730.10 \\
734.86 \\
741.35 \\
767.25\end{array}$ \\
\hline $\begin{array}{l}P-48 \\
P-47 \\
P-46 \\
P-45\end{array}$ & $\begin{array}{l}\text { Mudstone } \\
\text { Mudstone } \\
\text { Phosphate rock, argillaceous } \\
\text { Mudstone, phosphatic }\end{array}$ & $\begin{array}{l}1364-\text { RAH } \\
1363-\text { RAH } \\
1378-\text { WOM } \\
1377-\text { WOM }\end{array}$ & $\begin{array}{l}1.1 \\
3.6 \\
1.1 \\
0.6\end{array}$ & $\begin{array}{r}5.2 \\
5.6 \\
21.2 \\
8.6\end{array}$ & $\begin{array}{l}-- \\
-- \\
--\end{array}$ & $\begin{array}{l}-- \\
-- \\
--\end{array}$ & $\begin{array}{l}-- \\
-- \\
--\end{array}$ & $\begin{array}{l}71.4 \\
69.9 \\
32.2 \\
62.5\end{array}$ & $\begin{array}{l}54.0 \\
57.6 \\
58.7 \\
59.3\end{array}$ & $\begin{array}{l}772.97 \\
793.13 \\
816.45 \\
821.61\end{array}$ \\
\hline
\end{tabular}

Fossil collection made by J. E. Smedley, Paleontology and Stratigraphy Branch, U. S. Geological Survey. 


\begin{tabular}{|c|c|c|c|c|c|c|c|c|c|c|}
\hline \multirow[b]{2}{*}{$\begin{array}{c}\text { Bed } \\
\text { no. }\end{array}$} & \multirow[b]{2}{*}{ Rock description } & \multirow[b]{2}{*}{$\begin{array}{l}\text { Sample } \\
\text { no. }\end{array}$} & \multirow{2}{*}{$\begin{array}{l}\text { Thickness } \\
\text { (feet) }\end{array}$} & \multicolumn{5}{|c|}{ Chemical analyses (percent) } & \multirow{2}{*}{$\begin{array}{c}\text { Cumulative } \\
\text { thickness } \\
\text { (feet) }\end{array}$} & \multirow{2}{*}{$\begin{array}{l}\text { Thickness } \mathbf{x} \\
\text { percent } \mathrm{P}_{2} \mathrm{O}_{5} \\
\text { (cumulative) }\end{array}$} \\
\hline & & & & $\mathrm{P}_{2} \mathrm{O}_{5}$ & $\mathrm{Al}_{2} \mathrm{O}_{3}$ & $\mathrm{Fe}_{2} \mathrm{O}_{3}$ & $\begin{array}{l}\text { Loss on } \\
\text { ignition }\end{array}$ & $\begin{array}{c}\text { Acid } \\
\text { insoluble }\end{array}$ & & \\
\hline $\begin{array}{l}P-44 \\
P-43 \\
P-42 \\
P-41 \\
P-40\end{array}$ & $\begin{array}{l}\text { Phosphate rock, calcareous and } \\
\text { phosphatic mudstone } \\
\text { Mudstone } \\
\text { Phosphate rock, argillaceous } \\
\text { Mudstone } \\
\text { Mudstone and argillaceous phosphate } \\
\text { rock; fos. col. no. } 48 \text {-JES-107 }\end{array}$ & $\begin{array}{l}1376-\text { WOM } \\
1375-\text { WOM } \\
1374-\text { WOM } \\
1373-\text { WOM } \\
1370-\text { CEW }\end{array}$ & $\begin{array}{l}2.1 \\
0.7 \\
1.1 \\
0.8 \\
1.3\end{array}$ & $\begin{array}{r}17.7 \\
4.7 \\
22.4 \\
3.5 \\
11.2\end{array}$ & $\begin{array}{l}-- \\
-- \\
--\end{array}$ & $\begin{array}{l}-- \\
-- \\
-- \\
--\end{array}$ & $\begin{array}{l}-- \\
-- \\
-- \\
--\end{array}$ & $\begin{array}{l}33.5 \\
76.3 \\
36.2 \\
36.3 \\
54.4\end{array}$ & $\begin{array}{l}61.4 \\
62.1 \\
63.2 \\
64.0 \\
65.3\end{array}$ & $\begin{array}{l}858.78 \\
862.07 \\
886.71 \\
889.51 \\
904.07\end{array}$ \\
\hline $\begin{array}{l}P=39 \\
P-38 \\
P-37 \\
P=36 \\
P=35\end{array}$ & $\begin{array}{l}\text { Phosphate rock, argillaceous } \\
\text { Phosphate rock, argillaceous } \\
\text { Mudstone } \\
\text { Mudstone, phosphatic } \\
\text { Mudstone, phosphatic }\end{array}$ & $\begin{array}{l}1371-\text { CEW } \\
1372-\text { CEW } \\
1329-\text { WOM } \\
1328-W O M \\
1327-W O M\end{array}$ & $\begin{array}{l}2.7 \\
4.3 \\
1.8 \\
1.5 \\
0.4\end{array}$ & $\begin{array}{r}19.1 \\
15.5 \\
3.3 \\
9.2 \\
12.5\end{array}$ & $\begin{array}{l}-- \\
-- \\
-- \\
-- \\
--\end{array}$ & $\begin{array}{l}-- \\
-- \\
-- \\
-- \\
--\end{array}$ & $\begin{array}{l}-- \\
-- \\
-- \\
--\end{array}$ & $\begin{array}{l}31.0 \\
34.9 \\
73.3 \\
62.8 \\
52.8\end{array}$ & $\begin{array}{l}\text { 68. } 0 \\
72.3 \\
74.1 \\
75.6 \\
76.0\end{array}$ & $\begin{array}{r}955.64 \\
1,022.29 \\
1,028.23 \\
1,042.03 \\
1,047.03\end{array}$ \\
\hline $\begin{array}{l}P-34 \\
P-33 \\
P-32 \\
P-31 \\
P-30\end{array}$ & $\begin{array}{l}\text { Mudstone } \\
\text { Mudstone, phosphatic } \\
\text { Mudstone, phosphatic } \\
\text { Phosphate rock, argillaceous } \\
\text { Mudstone }\end{array}$ & $\begin{array}{l}1326-\text { WOM } \\
1325-\text { WOM } \\
1324-\text { WOM } \\
1323-\text { WOM } \\
1322-\text { WOM }\end{array}$ & $\begin{array}{l}2.0 \\
0.5 \\
0.7 \\
0.8 \\
0.7\end{array}$ & $\begin{array}{r}2.1 \\
13.2 \\
14.3 \\
20.7 \\
7.2\end{array}$ & $\begin{array}{l}-- \\
-- \\
-- \\
--\end{array}$ & $\begin{array}{l}-- \\
-- \\
-- \\
-- \\
--\end{array}$ & $\begin{array}{l}-- \\
-- \\
-- \\
--\end{array}$ & $\begin{array}{l}84.8 \\
50.4 \\
48.4 \\
34.3 \\
67.9\end{array}$ & $\begin{array}{l}78.0 \\
78.5 \\
79.2 \\
80.0 \\
80.7\end{array}$ & $\begin{array}{l}1,051.23 \\
1,057.83 \\
1,067.84 \\
1,084.40 \\
1,089.44\end{array}$ \\
\hline $\begin{array}{l}P-29 \\
P-28 \\
P-27 \\
P-26 \\
P-25\end{array}$ & $\begin{array}{l}\text { Phosphate rock and phosphatic } \\
\text { mudstone } \\
\text { Mudstone, phosphatic, calcareous } \\
\text { Mudstone, phosphatic, calcareous } \\
\text { Mudstone, phosphatic } \\
\text { Mudstone, phosphatic }\end{array}$ & $\begin{array}{l}1321-\text { WOM } \\
1527-\text { WOM } \\
1526-\text { WOM } \\
1362-\text { RAH } \\
1361-\text { RAH }\end{array}$ & $\begin{array}{l}0.9 \\
3.0 \\
3.0 \\
0.35 \\
3.4\end{array}$ & $\begin{array}{r}24.1 \\
13.8 \\
13.1 \\
7.9 \\
13.6\end{array}$ & $\begin{array}{l}-- \\
-- \\
-- \\
-- \\
--\end{array}$ & $\begin{array}{l}-- \\
-- \\
-- \\
--\end{array}$ & $\begin{array}{l}-- \\
-- \\
-- \\
-- \\
--\end{array}$ & $\begin{array}{l}24.9 \\
42.1 \\
41.0 \\
61.2 \\
46.0\end{array}$ & $\begin{array}{l}81.6 \\
84.6 \\
87.6 \\
87.95 \\
91.35\end{array}$ & $\begin{array}{l}1,111.13 \\
1,152.53 \\
1,191.83 \\
1,194.60 \\
1,240.84\end{array}$ \\
\hline $\begin{array}{l}P-24 \\
P-23 \\
P-22\end{array}$ & $\begin{array}{l}\text { Mudstone, phosphatic } \\
\text { Phosphate rock, argillaceous } \\
\text { Phosphate rock; fos. col. no. 48-JES-124 }\end{array}$ & $\begin{array}{l}1360-\text { RAH } \\
1393-\text { WOM } \\
1392-\text { WOM }\end{array}$ & $\begin{array}{l}0.6 \\
0.8 \\
3.0\end{array}$ & $\begin{array}{r}8.4 \\
16.8 \\
27.3\end{array}$ & $\begin{array}{l}-- \\
3.6\end{array}$ & $\begin{array}{l}-- \\
2.60 \\
1.77\end{array}$ & $\begin{array}{l}-- \\
8.94\end{array}$ & $\begin{array}{l}60.5 \\
41.2 \\
18.1\end{array}$ & $\begin{array}{l}91.95 \\
92.75 \\
95.75\end{array}$ & $\begin{array}{l}1,245.88 \\
1,259.32 \\
1,341.22\end{array}$ \\
\hline $\mathbf{P}-21$ & $\begin{array}{l}\text { Phosphate rock, argillaceous and phosphatic } \\
\text { mudstone }\end{array}$ & $1391-$ WOM & 2.7 & 21.2 & 3.9 & 1.41 & 10.38 & 30.9 & 98.45 & $1,398.46$ \\
\hline $\mathbf{P}-20$ & $\begin{array}{l}\text { Mudstone and argillaceous calcareous } \\
\text { phosphate rock }\end{array}$ & 1390-WOM & 2.8 & 13.6 & 5.9 & 2.11 & 13.32 & 43.4 & 101.25 & $1,436.54$ \\
\hline-- & $\begin{array}{l}\text { Mudstone and argillaceous phosphate rock } \\
1389-\text { WOM is equivalent to } 1390-\text { WOM. }\end{array}$ & $1389-W O M$ & $(3.0)$ & 18.3 & 5.3 & 1.78 & 12.30 & 36.9 & -- & -- \\
\hline $\begin{array}{l}P-19 \\
P-18 \\
P-17 \\
P-16\end{array}$ & $\begin{array}{l}\text { Phosphate rock and mudstone } \\
\text { Phosphate rock, argillaceous } \\
\text { Phosphate rock } \\
\text { Phosphate rock }\end{array}$ & $\begin{array}{l}1388-\text { WOM } \\
1387-\text { WOM } \\
1386-\text { WOM } \\
1359-\text { CEW }\end{array}$ & $\begin{array}{l}1.4 \\
4.0 \\
1.5 \\
1.05\end{array}$ & $\begin{array}{l}29.2 \\
20.6 \\
31.4 \\
28.5\end{array}$ & $\begin{array}{l}2.9 \\
5.5 \\
2.4 \\
2.3\end{array}$ & $\begin{array}{l}1.18 \\
1.99 \\
1.15 \\
0.85\end{array}$ & $\begin{array}{l}2.88 \\
8.24 \\
5.82 \\
7.76\end{array}$ & $\begin{array}{l}16.7 \\
36.1 \\
12.7 \\
15.3\end{array}$ & $\begin{array}{l}102.65 \\
106.65 \\
108.15 \\
109.20\end{array}$ & $\begin{array}{l}1,477.42 \\
1,559.82 \\
1,606.92 \\
1,636.84\end{array}$ \\
\hline $\begin{array}{l}P-15 \\
P-14 \\
P-13 \\
P-12\end{array}$ & $\begin{array}{l}\text { Phosphate rock } \\
\text { Phosphate rock and mudstone } \\
\text { Mudstone, phosphatic } \\
\text { Phosphate rock }\end{array}$ & $\begin{array}{l}1358-\mathrm{CEW} \\
1357-\mathrm{CEW} \\
1356-\mathrm{CEW} \\
1355-\mathrm{CEW}\end{array}$ & $\begin{array}{l}1.4 \\
0.9 \\
0.8 \\
2.3\end{array}$ & $\begin{array}{l}31.3 \\
27.4 \\
10.3 \\
31.3\end{array}$ & $\begin{array}{l}2.7 \\
3.5 \\
7.3 \\
2.0\end{array}$ & $\begin{array}{l}0.98 \\
1.25 \\
2.65 \\
0.92\end{array}$ & $\begin{array}{l}9.36 \\
6.60 \\
7.70 \\
6.62\end{array}$ & $\begin{array}{l}12.1 \\
20.7 \\
58.3 \\
13.0\end{array}$ & $\begin{array}{l}110.60 \\
111.50 \\
112.30 \\
114.60\end{array}$ & $\begin{array}{l}1,680.66 \\
1,705.32 \\
1,713.56 \\
1,785.55\end{array}$ \\
\hline
\end{tabular}




\begin{tabular}{|c|c|c|c|c|c|c|c|c|c|c|}
\hline $\begin{array}{l}\text { P-11 } \\
\text { P-10 } \\
\text { P- } 9 \\
\text { P- } 8 \\
\text { P- }\end{array}$ & $\begin{array}{l}\text { Mudstone, phosphatic } \\
\text { Phosphate rock } \\
\text { Mudstone, phosphatic } \\
\text { Phosphate rock, argillaceous } \\
\text { Mudstone }\end{array}$ & $\begin{array}{l}1354-\mathrm{CEW} \\
1353-\mathrm{CEW} \\
1352-\mathrm{CEW} \\
1351-\mathrm{CEW} \\
1350-\mathrm{CEW}\end{array}$ & $\begin{array}{l}1.0 \\
4.0 \\
0.45 \\
0.7 \\
0.8\end{array}$ & $\begin{array}{r}11.3 \\
34.5 \\
13.5 \\
24.5 \\
4.9\end{array}$ & $\begin{array}{l}7.9 \\
1.4 \\
7.8 \\
4.2 \\
1.7\end{array}$ & $\begin{array}{l}2.71 \\
0.56 \\
2.13 \\
1.54 \\
0.55\end{array}$ & $\begin{array}{l}7.16 \\
8.88 \\
8.42 \\
5.98 \\
8.08\end{array}$ & $\begin{array}{r}53.6 \\
5.9 \\
52.0 \\
25.6 \\
9.2\end{array}$ & $\begin{array}{l}115.60 \\
119.60 \\
120.05 \\
120.75 \\
121.55\end{array}$ & $\begin{array}{l}1,796.85 \\
1,934.85 \\
1,940.93 \\
1,958.08 \\
1,961.99\end{array}$ \\
\hline $\begin{array}{l}\because-6 \\
\dot{p}-5\end{array}$ & $\begin{array}{l}\text { Phosphate rock } \\
\text { Phosphate rock, calcareous, argillaceous; }\end{array}$ & $1000-$ RAH & 1.4 & 27.9 & 3.4 & 1.01 & 5.98 & 16.4 & 122.95 & $2,001.06$ \\
\hline $\begin{array}{l}P-4 \\
P-3 \\
P-2\end{array}$ & $\begin{array}{l}\text { fos, col. no. } 48-J E S-125 \\
\text { Phosphate rock } \\
\text { Phosphate rock } \\
\text { Mudstone, calcareous }\end{array}$ & $\begin{array}{l}\text { 1319- RAH } \\
1419-\text { WOM } \\
1233-\text { WOM } \\
1317-\text { RAH }\end{array}$ & $\begin{array}{l}0.7 \\
2.0 \\
3.0 \\
3.0\end{array}$ & $\begin{array}{r}15.7 \\
33.9 \\
32.5 \\
2.2\end{array}$ & $\begin{array}{l}3.0 \\
1.0 \\
1.1 \\
6.8\end{array}$ & $\begin{array}{l}1.06 \\
0.56 \\
0.93 \\
2.65\end{array}$ & $\begin{array}{r}6.70 \\
7.18 \\
8.06 \\
19.66\end{array}$ & $\begin{array}{r}21.1 \\
5.3 \\
6.6 \\
51.9\end{array}$ & $\begin{array}{l}123.65 \\
125.65 \\
128.65 \\
131.65\end{array}$ & $\begin{array}{l}2,012.05 \\
2,079.85 \\
2,177.35 \\
2,183.95\end{array}$ \\
\hline$P-1$ & Phosphate rock & 1316- RAH & 0.5 & 32.4 & 0.98 & 0.82 & 19.80 & 5.1 & 132.15 & $2,200.15$ \\
\hline \multicolumn{11}{|c|}{ Wells formation } \\
\hline $\mathrm{Cw}-1$ & Limestone & 1315- RAH & 3.1 & 0.6 & -- & -- & -- & 1.8 & -- & -- \\
\hline
\end{tabular}


Semi-quantitative analyses of selected samples of the phosphatic shale member of the Phosphoria formation, North Rasmussen Valley,

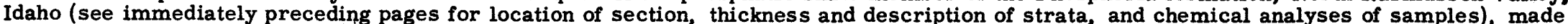

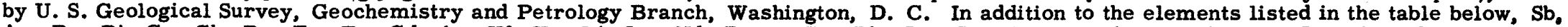
As, Be, Bi, Cs, Cb, Dy, Er, Eu, Gd, Au, Hf, Ho, Li, Lu, Nd, Pt, K, Pr, Rb, Sm, Sc, Ta, Te, Tb, Tl, Th, Tm, Sn, Ti, and W were looked for in all samples but were not detected.

\section{Explanation of symbols}

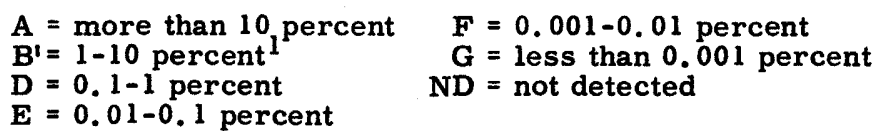

$F=0.001-0.01$ percent

$\mathbf{G}=$ less than 0.001 percent $\mathrm{ND}=$ not detected

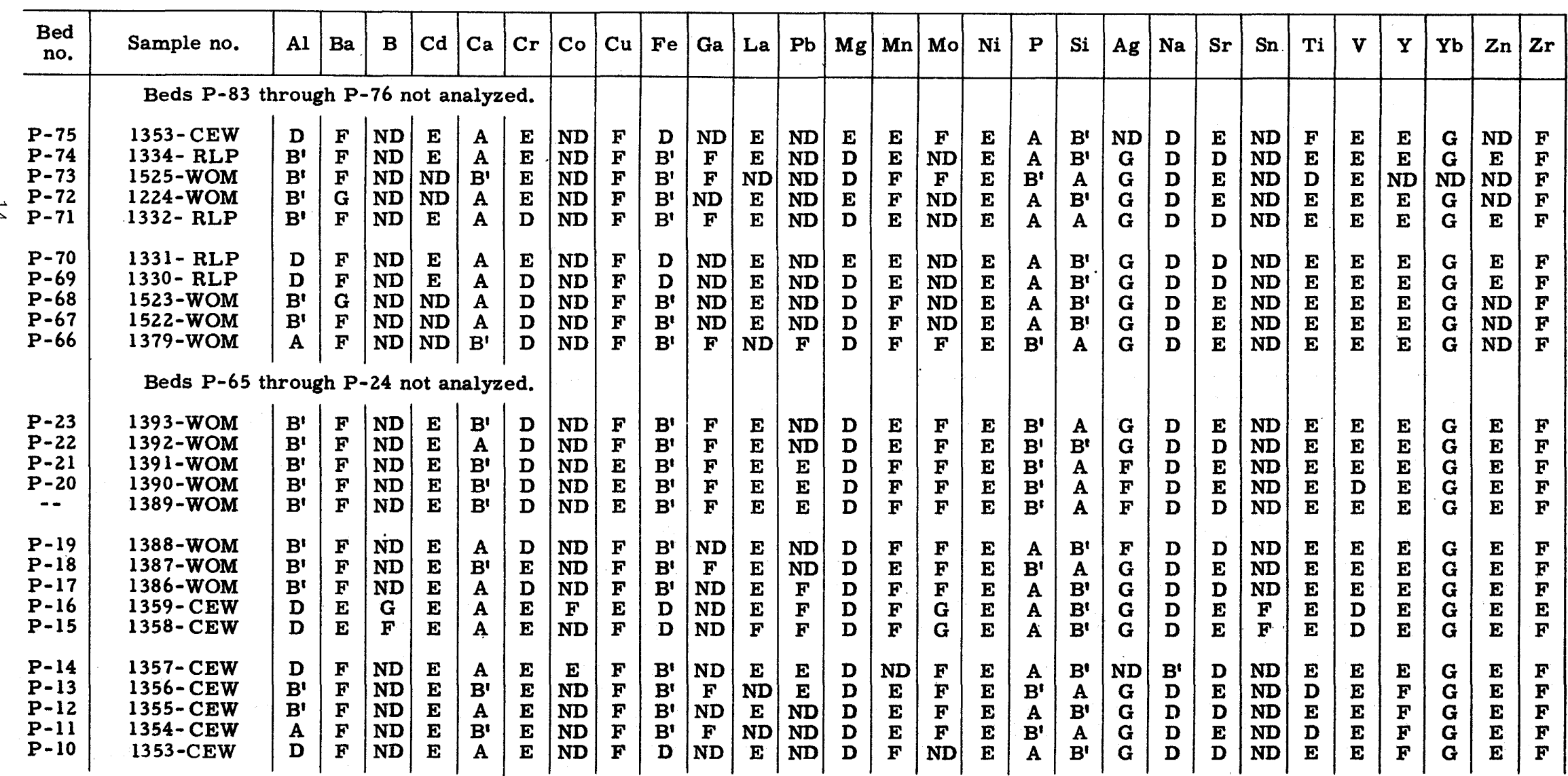




\begin{tabular}{|c|c|c|c|c|c|c|c|c|c|c|c|c|c|c|c|c|c|c|c|c|c|c|c|c|c|c|c|c|c|}
\hline $\begin{array}{l}P-9 \\
P=8 \\
P=7 \\
P-6 \\
P-5\end{array}$ & $\begin{array}{l}1352-\mathrm{CEW} \\
1351-\mathrm{CEW} \\
1350-\mathrm{CEW} \\
1000-\mathrm{RAH} \\
1319-\mathrm{RAH}\end{array}$ & $\begin{array}{c}A \\
B^{t} \\
D \\
B^{\prime} \\
B^{t}\end{array}$ & $\begin{array}{l}\mathbf{F} \\
\mathbf{F} \\
\mathbf{F} \\
\mathbf{E} \\
\mathbf{F}\end{array}$ & $\begin{array}{l}\text { ND } \\
\text { ND } \\
\text { ND } \\
\text { F } \\
\text { ND }\end{array}$ & $\begin{array}{l}\mathbf{E} \\
\mathbf{E} \\
\mathbf{E} \\
\mathbf{E} \\
\mathbf{E}\end{array}$ & $\begin{array}{l}\mathbf{B}^{\mathbf{\prime}} \\
\mathbf{A} \\
\mathbf{A} \\
\mathbf{A} \\
\text { A }\end{array}$ & $\begin{array}{l}\mathbf{E} \\
\mathbf{E} \\
\mathbf{E} \\
\mathbf{E} \\
\mathbf{E}\end{array}$ & $\begin{array}{l}\text { ND } \\
\text { ND } \\
\text { ND } \\
\text { ND } \\
\text { ND }\end{array}$ & $\begin{array}{l}\mathbf{F} \\
\mathbf{F} \\
\mathbf{F} \\
\mathbf{E} \\
\mathbf{F}\end{array}$ & $\begin{array}{c}\mathbf{B}^{\prime} \\
\mathbf{B}^{\prime} \\
\mathbf{D} \\
\mathbf{D} \\
\mathbf{B}^{\mathbf{t}}\end{array}$ & $\begin{array}{l}F \\
F \\
\text { ND } \\
\text { ND } \\
\text { DD }\end{array}$ & $\begin{array}{c}\text { ND } \\
\mathbf{E} \\
\mathrm{ND} \\
\mathrm{E} \\
\mathbf{E}\end{array}$ & $\begin{array}{c}\text { ND } \\
\text { ND } \\
\mathbf{F} \\
\mathbf{F} \\
\text { ND }\end{array}$ & $\begin{array}{l}\text { D } \\
\text { D } \\
\text { A } \\
\text { D } \\
\mathbf{B}^{:}\end{array}$ & $\begin{array}{l}\mathbf{E} \\
\mathbf{E} \\
\mathbf{E} \\
\mathbf{F} \\
\mathbf{E}\end{array}$ & $\begin{array}{l}\mathbf{F} \\
\mathbf{F} \\
\mathbf{F} \\
\mathbf{F} \\
\mathbf{F}\end{array}$ & $\begin{array}{l}\mathbf{E} \\
\mathbf{E} \\
\mathbf{E} \\
\mathbf{E} \\
\mathbf{E}\end{array}$ & $\begin{array}{l}\mathbf{B}^{\prime} \\
\mathbf{B}^{\mathbf{s}} \\
\mathbf{D} \\
\mathbf{A} \\
\mathbf{B}^{\mathbf{c}}\end{array}$ & $\begin{array}{l}\mathbf{A} \\
\mathbf{B}^{\prime} \\
\mathbf{B}^{\prime} \\
\mathbf{B}^{\prime} \\
\mathbf{B}^{\prime}\end{array}$ & $\begin{array}{l}\mathbf{G} \\
\mathbf{G} \\
\mathbf{G} \\
\mathbf{G} \\
\mathbf{G}\end{array}$ & $\begin{array}{l}\text { D } \\
\text { D } \\
\text { D } \\
\text { D } \\
\text { D }\end{array}$ & $\begin{array}{l}\mathbf{E} \\
\mathbf{D} \\
\mathbf{E} \\
\mathbf{E} \\
\mathbf{E}\end{array}$ & $\begin{array}{l}\text { ND } \\
\text { ND } \\
\text { ND } \\
\text { ND } \\
\text { ND }\end{array}$ & $\begin{array}{l}\mathbf{E} \\
\mathbf{E} \\
\mathbf{F} \\
\mathbf{E} \\
\mathbf{E}\end{array}$ & $\begin{array}{l}\mathbf{E} \\
\mathbf{E} \\
\mathbf{E} \\
\mathbf{D} \\
\mathbf{E}\end{array}$ & $\begin{array}{l}\mathbf{F} \\
\mathbf{F} \\
\mathbf{F} \\
\mathbf{E} \\
\mathbf{F}\end{array}$ & $G$ & $\begin{array}{l}\mathbf{E} \\
\mathbf{E} \\
\mathbf{E} \\
\mathbf{E} \\
\mathbf{E}\end{array}$ & $\begin{array}{l}\mathbf{F} \\
\mathbf{F} \\
\mathbf{F} \\
\mathbf{F} \\
\mathbf{F}\end{array}$ \\
\hline $\begin{array}{l}P-4 \\
P-3 \\
P-2 \\
P-1\end{array}$ & $\begin{array}{l}1419-\text { WOM } \\
1233-\text { WOM } \\
1317-\text { RAH } \\
1316-\text { RAH }\end{array}$ & $\begin{array}{l}\mathbf{D} \\
\mathbf{D} \\
\mathbf{B}^{\prime} \\
\mathbf{B}^{\prime}\end{array}$ & $\begin{array}{l}\mathbf{G} \\
\mathbf{F} \\
\mathbf{F} \\
\mathbf{F}\end{array}$ & $\begin{array}{l}\mathrm{ND} \\
\mathrm{ND} \\
\mathrm{ND} \\
\mathrm{ND}\end{array}$ & $\begin{array}{l}\mathbf{E} \\
\mathbf{E} \\
\mathbf{E} \\
\mathbf{E}\end{array}$ & $\begin{array}{l}\mathbf{A} \\
\mathbf{A} \\
\mathbf{B}^{\prime} \\
\mathbf{A}\end{array}$ & $\begin{array}{l}\mathbf{E} \\
\mathbf{D} \\
\mathbf{E} \\
\mathbf{E}\end{array}$ & $\begin{array}{l}\text { ND } \\
\text { ND } \\
\text { ND } \\
\text { ND }\end{array}$ & $\begin{array}{l}\mathbf{F} \\
\mathbf{F} \\
\mathbf{F} \\
\mathbf{F}\end{array}$ & $\begin{array}{l}\mathbf{D} \\
\mathbf{B}^{\prime} \\
\mathbf{B}^{\prime} \\
\mathbf{B}^{\prime}\end{array}$ & $\begin{array}{l}\text { ND } \\
\text { ND } \\
\text { F } \\
\text { ND }\end{array}$ & $\begin{array}{c}\mathbf{E} \\
\mathbf{E} \\
\mathbf{N D} \\
\mathbf{E}\end{array}$ & $\begin{array}{l}\text { ND } \\
\text { E } \\
\text { ND } \\
\text { ND }\end{array}$ & $\begin{array}{l}D \\
D \\
B^{\prime} \\
D\end{array}$ & $\begin{array}{l}\mathbf{F} \\
\mathbf{F} \\
\mathbf{E} \\
\mathbf{E}\end{array}$ & $\begin{array}{c}\text { ND } \\
F \\
\text { F } \\
\text { ND }\end{array}$ & $\begin{array}{l}\mathbf{E} \\
\mathbf{E} \\
\mathbf{D} \\
\mathbf{E}\end{array}$ & $\begin{array}{l}\text { A } \\
\text { A } \\
\text { D } \\
\text { A }\end{array}$ & $\begin{array}{l}\mathbf{D} \\
\mathbf{B}^{\prime} \\
\mathbf{A} \\
\mathbf{B}^{\prime}\end{array}$ & $\begin{array}{c}\mathbf{G} \\
\mathbf{G} \\
\mathbf{N D} \\
\mathbf{G}\end{array}$ & $\begin{array}{l}\text { D } \\
B^{\prime} \\
D \\
D\end{array}$ & $\begin{array}{l}\mathbf{E} \\
\mathbf{E} \\
\mathbf{E} \\
\mathbf{D}\end{array}$ & $\begin{array}{l}\text { ND } \\
\text { ND } \\
\text { ND } \\
\text { ND }\end{array}$ & $\begin{array}{l}\mathbf{F} \\
\mathbf{E} \\
\mathbf{E} \\
\mathbf{E}\end{array}$ & $\begin{array}{l}\mathbf{E} \\
\mathbf{E} \\
\mathbf{E} \\
\mathbf{E}\end{array}$ & $\begin{array}{l}\mathbf{E} \\
\mathbf{E} \\
\mathbf{F} \\
\mathbf{F}\end{array}$ & $\mathbf{G}$ & $\begin{array}{l}\mathbf{E} \\
\mathbf{E} \\
\mathbf{E} \\
\mathbf{E}\end{array}$ & $\begin{array}{l}\mathbf{F} \\
\mathbf{F} \\
\mathbf{F} \\
\mathbf{F}\end{array}$ \\
\hline
\end{tabular}

${ }^{1} B^{\prime}$ is equivalent to $B$ and $C$ of the Bureau of Mines analyses as recorded in other reports. 
SOUTH RASMUSSEN VALLEY, IDAHO. LOT NO. 1232.

Phosphatic shale member of Phosphoria formation sampled in bulldozer trench on northeast slope of Rasmussen Valley, sec. 9, T. 7 S.,

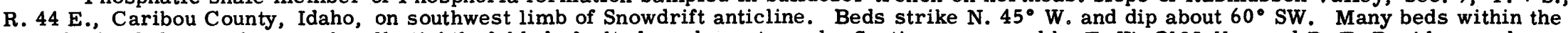
phosphatic shale member are locally tightly folded, faulted, and overturned. Section measured by F. W. O' Malley and D. F. Davidson and

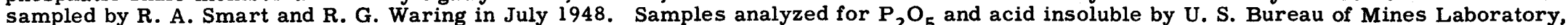
Albany, Oregon, and for other constituents by Trace Elements Section Laboratory, ${ }^{5}$. S. Geological Survey, Washington, D. C.

\begin{tabular}{|c|c|c|c|c|c|c|c|c|c|c|}
\hline \multirow{2}{*}{$\begin{array}{c}\text { Bed } \\
\text { no. }\end{array}$} & \multirow{2}{*}{ Rock description } & \multirow{2}{*}{$\begin{array}{c}\text { Sample } \\
\text { no. }\end{array}$} & \multirow{2}{*}{$\begin{array}{c}\text { Thickness } \\
\text { (feet) }\end{array}$} & \multicolumn{5}{|c|}{ Chemical analyses (percent) } & \multirow{2}{*}{$\begin{array}{l}\text { Cumulative } \\
\text { thickness } \\
\text { (feet) }\end{array}$} & \multirow{2}{*}{$\begin{array}{l}\text { Thickness } x \\
\text { percent } \mathrm{P}_{2} \mathrm{O}_{5} \\
\text { (cumulative) }\end{array}$} \\
\hline & & & & $\mathrm{P}_{2} \mathrm{O}_{5}$ & $\mathrm{Al}_{2} \mathrm{O}_{3}$ & $\mathrm{Fe}_{2} \mathrm{O}_{3}$ & $\begin{array}{l}\text { Loss on } \\
\text { ignition }\end{array}$ & $\begin{array}{c}\text { Acid } \\
\text { insoluble }\end{array}$ & & \\
\hline \multicolumn{11}{|c|}{ Rex member of Phosphoria formation-basal beds only } \\
\hline $\begin{array}{l}R-6 \\
R-5 \\
R-4 \\
R-3 \\
R-2\end{array}$ & $\begin{array}{l}\text { Chert } \\
\text { Chert } \\
\text { Chert } \\
\text { Chert } \\
\text { Chert }\end{array}$ & $\begin{array}{l}1492-\text { DFD } \\
1491-\text { DFD } \\
1490-\text { DFD } \\
1474-\text { DFD } \\
1473-\text { DFD }\end{array}$ & $\begin{array}{l}0.8 \\
1.8 \\
1.2 \\
1.4 \\
2.3\end{array}$ & $\begin{array}{l}0.6 \\
0.6 \\
0.5 \\
0.4 \\
0.1\end{array}$ & $\begin{array}{l}-- \\
-- \\
-- \\
--\end{array}$ & $\begin{array}{l}-- \\
-- \\
-- \\
--\end{array}$ & $\begin{array}{l}-- \\
-- \\
-- \\
--\end{array}$ & $\begin{array}{l}94.4 \\
94.4 \\
94.5 \\
92.9 \\
92.7\end{array}$ & $\begin{array}{l}0.8 \\
2.6 \\
3.8 \\
5.2 \\
7.5\end{array}$ & $\begin{array}{l}0.48 \\
1.56 \\
2.16 \\
2.72 \\
2.95\end{array}$ \\
\hline $\mathbf{R}-\mathbf{1}$ & Chert & 1472- DFD & 1.5 & 4.4 & -- & -- & $-\infty$ & 74.6 & 9.0 & 9.55 \\
\hline \multicolumn{11}{|c|}{ Phosphatic shale member of Phosphoria formation } \\
\hline $\begin{array}{l}P-72 \\
P-71 \\
P-70 \\
P-69 \\
P-68\end{array}$ & $\begin{array}{l}\text { Mudstone, cherty } \\
\text { Mudstone } \\
\text { Mudstone } \\
\text { Phosphate rock, argillaceous } \\
\text { Mudstone }\end{array}$ & $\begin{array}{l}3190-\text { WOM } \\
3189-\text { WOM } \\
3188-\text { WOM } \\
3187-\text { WOM } \\
3186-\text { WOM }\end{array}$ & $\begin{array}{l}0.9 \\
0.7 \\
1.2 \\
0.8 \\
1.7\end{array}$ & $\begin{array}{r}2.7 \\
4.5 \\
3.7 \\
25.9 \\
1.5\end{array}$ & $\begin{array}{l}-- \\
-- \\
-- \\
--\end{array}$ & $\begin{array}{l}-- \\
-- \\
-- \\
--\end{array}$ & $\begin{array}{l}-- \\
-- \\
-- \\
--\end{array}$ & $\begin{array}{l}83.2 \\
72.2 \\
73.6 \\
28.5 \\
79.2\end{array}$ & $\begin{array}{l}0.9 \\
1.6 \\
2.8 \\
3.6 \\
5.3\end{array}$ & $\begin{array}{r}2.43 \\
5.58 \\
10.02 \\
30.74 \\
33.29\end{array}$ \\
\hline $\begin{array}{l}P-67 \\
P-66 \\
P-65 \\
P-64 \\
P-63\end{array}$ & $\begin{array}{l}\text { Mudstone } \\
\text { Phosphate rock, argillaceous } \\
\text { Mudstone } \\
\text { Mudstone } \\
\text { Mudstone, calcareous, phosphatic }\end{array}$ & $\begin{array}{l}3185-\text { WOM } \\
3184-\text { WOM } \\
3183-\text { WOM } \\
1459-\text { DFD } \\
1458-\text { DFD }\end{array}$ & $\begin{array}{l}1.7 \\
0.3 \\
0.9 \\
0.7 \\
1.1\end{array}$ & $\begin{array}{r}0.6 \\
21.7 \\
1.3 \\
6.9 \\
26.6\end{array}$ & $\begin{array}{l}-- \\
-- \\
-- \\
-- \\
3.3\end{array}$ & $\begin{array}{l}-- \\
-- \\
-- \\
-\overline{1.01}\end{array}$ & $\begin{array}{l}-- \\
-- \\
-- \\
-- \\
5.74\end{array}$ & $\begin{array}{l}83.8 \\
34.8 \\
80.9 \\
67.9 \\
20.8\end{array}$ & $\begin{array}{r}7.0 \\
7.3 \\
8.2 \\
8.9 \\
10.0\end{array}$ & $\begin{array}{l}34.31 \\
40.82 \\
41.99 \\
46.82 \\
78.06\end{array}$ \\
\hline $\begin{array}{l}P-62 \\
P-61 \\
P-60 \\
P-59 \\
P-58\end{array}$ & $\begin{array}{l}\text { Phosphate rock } \\
\text { Phosphate rock } \\
\text { Phosphate rock } \\
\text { Phosphate rock, argillaceous } \\
\text { Phosphate rock, argillaceous }\end{array}$ & $\begin{array}{l}1457-\text { DFD } \\
1456-\text { DFD } \\
1455-\text { DFD } \\
1454-\text { DFD } \\
1453-\text { DFD }\end{array}$ & $\begin{array}{l}1.1 \\
1.0 \\
1.4 \\
1.0 \\
2.6\end{array}$ & $\begin{array}{l}36.0 \\
30.1 \\
29.8 \\
25.8 \\
27.1\end{array}$ & $\begin{array}{l}1.4 \\
2.6 \\
3.2 \\
3.8 \\
3.4\end{array}$ & $\begin{array}{l}0.53 \\
0.80 \\
0.80 \\
1.24 \\
1.15\end{array}$ & $\begin{array}{r}3.24 \\
9.28 \\
37.00 \\
9.88 \\
8.84\end{array}$ & $\begin{array}{l}3.6 \\
12.0 \\
12.0 \\
21.1 \\
20.1\end{array}$ & $\begin{array}{l}11.1 \\
12.1 \\
13.5 \\
14.5 \\
17.1\end{array}$ & $\begin{array}{l}115.68 \\
145.78 \\
187.50 \\
213.30 \\
283.76\end{array}$ \\
\hline $\begin{array}{l}P-57 \\
P-56 \\
P=55 \\
P-54 \\
P-53\end{array}$ & $\begin{array}{l}\text { Phosphate rock, argillaceous } \\
\text { Phosphate rock, argillaceous } \\
\text { Phosphate rock, argillaceous } \\
\text { Mudstone, phosphatic } \\
\text { Mudstone, calcareous }\end{array}$ & $\begin{array}{l}1452-\text { DFD } \\
1451-\text { DFD } \\
1450-\text { DFD } \\
1439-\text { DFD } \\
1438-\text { DFD }\end{array}$ & $\begin{array}{l}0.5 \\
0.6 \\
0.6 \\
1.3 \\
0.6\end{array}$ & $\begin{array}{r}27.2 \\
20.0 \\
17.8 \\
14.6 \\
8.4\end{array}$ & $\begin{array}{l}3.1 \\
5.7 \\
6.7 \\
8.3 \\
-.\end{array}$ & $\begin{array}{c}0.85 \\
1.31 \\
1.22 \\
1.95 \\
--\end{array}$ & $\begin{array}{l}8.38 \\
9.72 \\
9.86 \\
9.74 \\
--\end{array}$ & $\begin{array}{l}20.5 \\
36.0 \\
40.4 \\
45.3 \\
62.5\end{array}$ & $\begin{array}{l}17.6 \\
18.2 \\
18.8 \\
20.1 \\
20.7\end{array}$ & $\begin{array}{l}297.36 \\
309.36 \\
320.04 \\
339.02 \\
344.06\end{array}$ \\
\hline $\begin{array}{l}P-52 \\
P-51 \\
P=50 \\
P-49\end{array}$ & $\begin{array}{l}\text { Mudstone } \\
\text { Mudstone, phosphatic } \\
\text { Mudstone, phosphatic } \\
\text { Mudstone, phosphatic }\end{array}$ & $\begin{array}{l}1437-\text { DFD } \\
1479-\text { DFD } \\
1478-\text { DFD } \\
1477-\text { DFD }\end{array}$ & $\begin{array}{l}1.3 \\
1.1 \\
0.7 \\
1.2\end{array}$ & $\begin{array}{r}5.4 \\
13.2 \\
8.7 \\
15.8\end{array}$ & $\begin{array}{l}-- \\
-- \\
--\end{array}$ & $\begin{array}{l}-- \\
-- \\
--\end{array}$ & $\begin{array}{l}-- \\
-- \\
--\end{array}$ & $\begin{array}{l}66.7 \\
44.8 \\
58.7 \\
43.2\end{array}$ & $\begin{array}{l}22.0 \\
23.1 \\
23.8 \\
25.0\end{array}$ & $\begin{array}{l}351.08 \\
365.60 \\
371.69 \\
390.65\end{array}$ \\
\hline
\end{tabular}




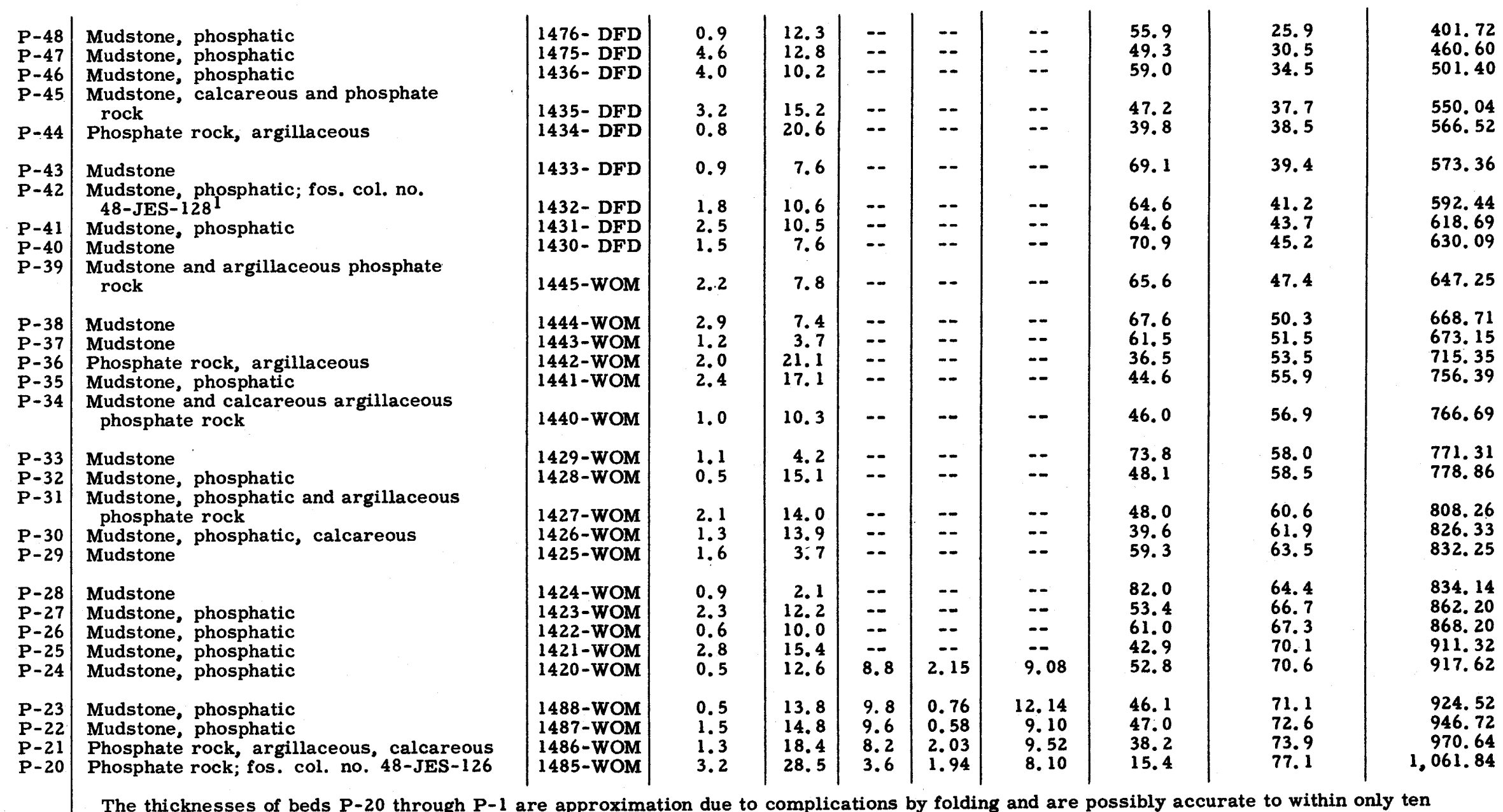

The thicknesses of beds P-20 through P-1 are approximation due to complications by folding and are possibly accurate to within only ten percent.

P-19 Mudstone, phosphatic, and argillaceous phosphate rock

P-18 . Phosphate rock, argillaceous

P-17 Mudstone, phosphatic

P-16 Mudstone, phosphatic

P-15 Phosphate rock, argillaceous

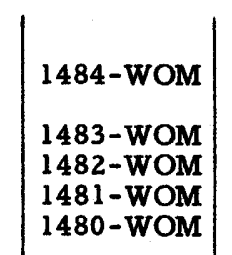

\begin{tabular}{l|r|r|r|r}
$2.5 ?$ & 16.6 & 5.0 & 1.30 & 11.10 \\
0.3 & 18.6 & 4.8 & 5.08 & 6.80 \\
$1.7 ?$ & 12.3 & 8.5 & 4.12 & 8.20 \\
0.6 & 16.7 & 7.7 & 1.80 & 4.96 \\
0.5 & 23.7 & 4.6 & 0.86 & 3.94
\end{tabular}

41.3
38.1
50.2
47.7
32.8

79.6

$1,103.34$

79.9

81.6

82. 2

82. 7

$1,108.92$

$1,129.83$

$1,139.85$

$1,151.70$

\footnotetext{
${ }^{1}$ Fossil collection made by J. E. Smedley, Paleontology and Stratigraphy Branch, U. S. Geological Survey.
} 


\begin{tabular}{|c|c|c|c|c|c|c|c|c|c|c|}
\hline \multirow[b]{2}{*}{$\begin{array}{c}\text { Bed } \\
\text { no. }\end{array}$} & \multirow[b]{2}{*}{ Rock description } & \multirow{2}{*}{$\begin{array}{c}\text { Sample } \\
\text { no. }\end{array}$} & \multirow{2}{*}{$\begin{array}{c}\text { Thickness } \\
\text { (feet) }\end{array}$} & \multicolumn{5}{|c|}{ Chemical analyses (percent) } & \multirow{2}{*}{$\begin{array}{c}\text { Cumulative } \\
\text { thickness } \\
\text { (feet) }\end{array}$} & \multirow{2}{*}{$\begin{array}{l}\text { Thickness } x \\
\text { percent } \mathrm{P}_{2} \mathrm{O}_{5} \\
\text { (cumulative) }\end{array}$} \\
\hline & & & & $\mathbf{P}_{2} \mathrm{O}_{5}$ & $\mathrm{Al}_{2} \mathrm{O}_{3}$ & $\mathrm{Fe}_{2} \mathrm{O}_{3}$ & $\begin{array}{l}\text { Loss on } \\
\text { ignition }\end{array}$ & $\begin{array}{c}\text { Acid } \\
\text { insoluble }\end{array}$ & & \\
\hline $\begin{array}{l}P-14 \\
P-13 \\
P-12 \\
P-11 \\
P-10\end{array}$ & $\begin{array}{l}\text { Phosphate rock } \\
\text { Mudstone, phosphatic } \\
\text { Phosphate rock } \\
\text { Phosphate rock }\end{array}$ & $\begin{array}{l}1469-\text { WOM } \\
1468-\text { WOM } \\
1489-\text { WOM } \\
1467-\text { WOM } \\
1466-\text { WOM }\end{array}$ & $\begin{array}{l}4.0 ? \\
0.5 \\
4.7 \\
2.3 \\
0.3\end{array}$ & $\begin{array}{l}32.2 \\
13.7 \\
36.3 \\
32.0 \\
12.4\end{array}$ & $\begin{array}{l}2.2 \\
7.9 \\
1.1 \\
1.9 \\
8.2\end{array}$ & $\begin{array}{l}0.63 \\
1.00 \\
0.38 \\
0.48 \\
0.68\end{array}$ & $\begin{array}{r}10.02 \\
6.04 \\
3.34 \\
5.24 \\
6.00\end{array}$ & $\begin{array}{r}13.0 \\
45.8 \\
5.1 \\
24.6\end{array}$ & $\begin{array}{l}86.7 \\
87.2 \\
91.9 \\
94.2 \\
94.5\end{array}$ & $\begin{array}{l}1,280.50 \\
1,287.35 \\
1,457.96 \\
1,531.56\end{array}$ \\
\hline $\begin{array}{l}P-9 \\
P-8 \\
P-7 \\
P-6 \\
P=5\end{array}$ & $\begin{array}{l}\text { Phosphate rock, argillaceous, calcareous, } \\
\text { and phosphatic mudstone } \\
\text { Mudstone, calcareous, phosphatic } \\
\text { Phosphate rock } \\
\text { Phosphate rock, argillaceous } \\
\text { Phosphate rock; fos. col. no. } 48 \text {-JES-127 }\end{array}$ & $\begin{array}{l}1465-\text { WOM } \\
1464-\text { WOM } \\
1463-\text { WOM } \\
1462-\text { WOM } \\
1461-W O M\end{array}$ & $\begin{array}{l}1.6 ? \\
0.7 \\
4.5 \\
0.4 \\
1.8 ?\end{array}$ & $\begin{array}{l}28.2 \\
12.1 \\
28.0 \\
24.3 \\
32.1\end{array}$ & $\begin{array}{r}2.9 \\
12.0 \\
2.4 \\
4.3 \\
1.3\end{array}$ & $\begin{array}{l}0.58 \\
4.29 \\
0.70 \\
0.72 \\
0.46\end{array}$ & $\begin{array}{l}4.96 \\
9.92 \\
6.38 \\
6.20 \\
5.04\end{array}$ & $\begin{array}{r}19.7 \\
37.5 \\
14.0 \\
37.1 \\
6.7\end{array}$ & $\begin{array}{r}96.1 \\
96.8 \\
101.3 \\
101.7 \\
103.5\end{array}$ & $\begin{array}{l}1,580.40 \\
1,588.87 \\
1,714.87 \\
1,724.59 \\
1,782.37\end{array}$ \\
\hline $\begin{array}{l}P-4 \\
P-3 \\
P-2 \\
P-1\end{array}$ & $\begin{array}{l}\text { Phosphate rock, calcareous } \\
\text { Phosphate rock } \\
\text { Mudstone, calcareous } \\
\text { Phosphate rock, calcareous }\end{array}$ & $\begin{array}{l}1460-\text { WOM } \\
1449-\text { WOM } \\
1448-\text { WOM } \\
1447-\text { WOM }\end{array}$ & $\begin{array}{l}0.4 ? \\
1.1 \\
4.0 ? \\
0.5\end{array}$ & $\begin{array}{r}29.9 \\
32.2 \\
1.7 \\
1.7\end{array}$ & $\begin{array}{l}1.6 \\
2.6 \\
9.2 \\
1.2\end{array}$ & $\begin{array}{l}0.57 \\
0.48 \\
2.78 \\
7.54\end{array}$ & $\begin{array}{l}7.26 \\
5.74 \\
8.60 \\
5.20\end{array}$ & $\begin{array}{l}8.7 \\
14.7 \\
71.8 \\
54.9\end{array}$ & $\begin{array}{l}103.9 \\
105.0 \\
109.0 \\
109.5\end{array}$ & $\begin{array}{l}1,794.33 \\
1,829.75 \\
1,836.55 \\
1,837.40\end{array}$ \\
\hline \multicolumn{11}{|c|}{ Wells formation } \\
\hline Cw-1 & Mudstone, calcareous & 1446-WOM & 3.5 & 1.3 & - & -- & -- & 67.0 & 3.5 & 4.55 \\
\hline
\end{tabular}


SPECTROGRAPHIC ANALYSES-SOUTH RASMUSSEN VALLEY, IDAHO. LOT NO. 1232.

Semi-quantitative analyses of selected samples of phosphatic shale member of Phosphopia formation, South Rasmussen Valley, Idaho (see immediately preceding pages for location of section, thickness and description of strata, and chemical analyses of samples), made by U. S. Geological Survey Laboratory, Geochemistry and Petrology Branch, Washington, D. C. In addition to the elements listed in the table below, Sb, As, Be, Bi, Ce, Cs, Co, Cb, Dy, Er, Eu, Gd, Ge, Au, Ho, Li, Lu, Nd, Pt, Pr, Rb, Sm, Sc, Ta, Te, Tb, Tl, Tm, and W were looked for in all samples but were not detected.

Explanation of symbols

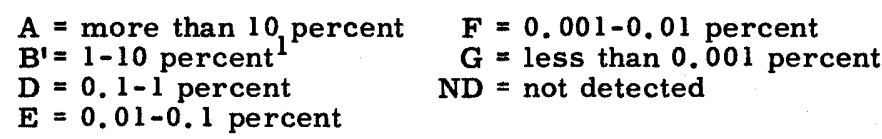

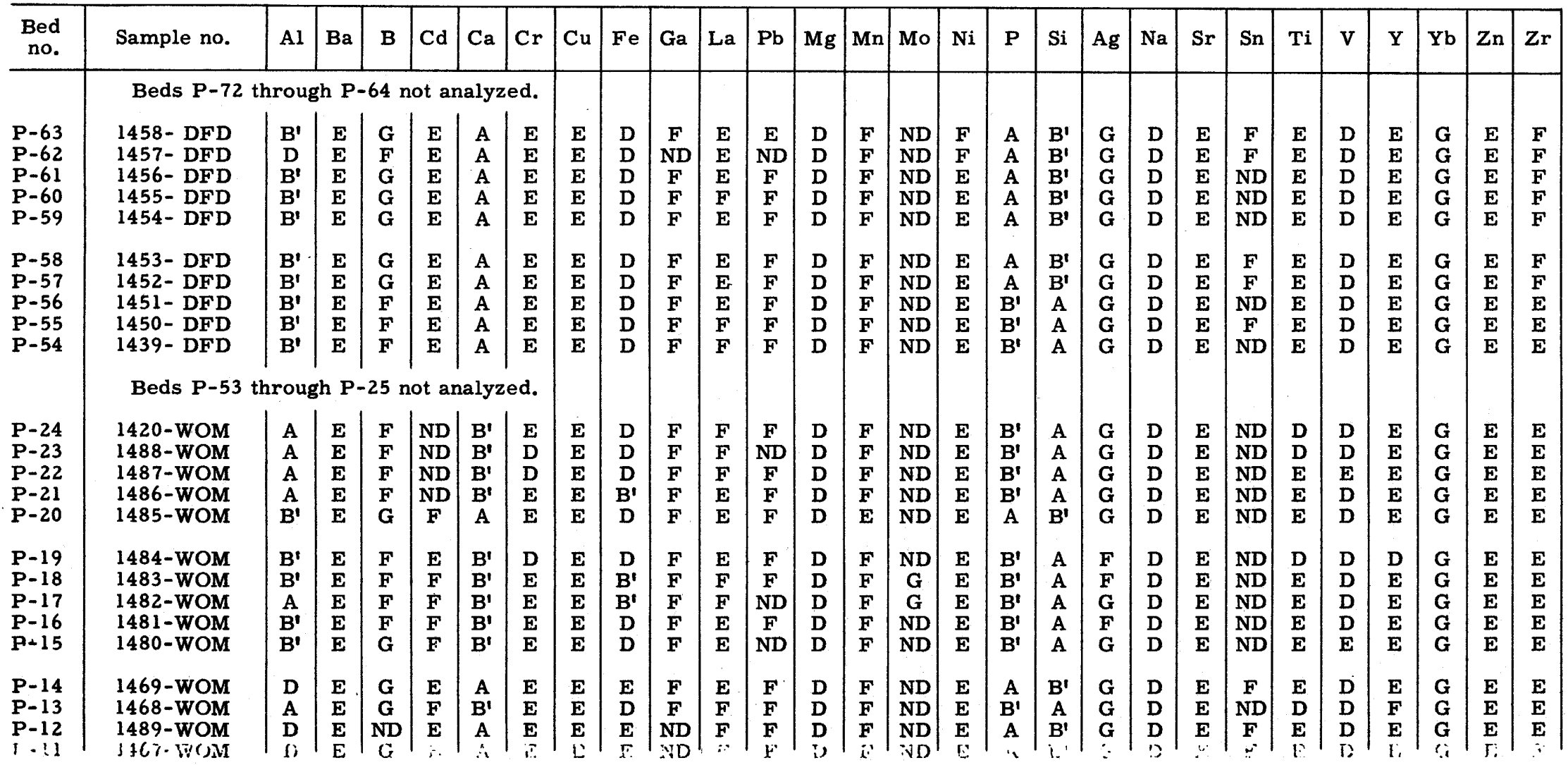

${ }^{1} B^{\prime}$ is equivalent to $B$ and $C$ of Bureau of Mines analyses as recorded in other reports. 


\begin{tabular}{|c|c|c|c|c|c|c|c|c|c|c|c|c|c|c|c|c|c|c|c|c|c|c|c|c|c|c|c|c|}
\hline $\begin{array}{c}\text { Bed } \\
\text { no. }\end{array}$ & Sample no. & Al & $\mathbf{B a}$ & B & Cd & $\mathrm{Ca}$ & $\mathrm{Cr}$ & $\mathrm{Cu}$ & $\mathrm{Fe}$ & Ga & $\mathbf{L a}$ & $\mathbf{P b}$ & $\mathbf{M g}$ & Mn & Mo & $\mathrm{Ni}$ & $\mathbf{P}$ & $\mathbf{S i}$ & $\mathbf{A g}$ & $\mathrm{Na}$ & $\mathrm{Sr}$ & Sn & $\mathrm{Ti}$ & $\mathbf{v}$ & $\mathbf{Y}$ & $\mathbf{Y b}$ & $\mathbf{Z n}$ & $\mathbf{Z r}$ \\
\hline $\begin{array}{l}\text { P-11 } \\
\text { P-10 } \\
\text { P- } 9 \\
\text { P- } 8 \\
\text { P- } 7\end{array}$ & $\begin{array}{l}1467-\text { WOM } \\
1466-\text { WOM } \\
1464-\text { WOM } \\
1464-\text { WOM } \\
1463-\text { WOM }\end{array}$ & $\begin{array}{l}\mathbf{D} \\
\mathbf{A} \\
\mathbf{B}^{\prime} \\
\mathbf{A} \\
\mathbf{B}^{\mathbf{\prime}}\end{array}$ & $\begin{array}{l}\mathbf{E} \\
\mathbf{E} \\
\mathbf{E} \\
\mathbf{E} \\
\mathbf{E}\end{array}$ & $\begin{array}{l}\mathbf{G} \\
\mathbf{G} \\
\mathbf{G} \\
\mathbf{F} \\
\mathbf{G}\end{array}$ & $\begin{array}{l}\mathbf{E} \\
\mathbf{F} \\
\mathbf{E} \\
\mathbf{F} \\
\mathbf{E}\end{array}$ & $\begin{array}{c}\mathbf{A} \\
\mathbf{B}^{\prime} \\
\mathbf{A} \\
\mathbf{B}^{\prime} \\
\mathbf{A}\end{array}$ & $\begin{array}{l}\mathbf{E} \\
\mathbf{E} \\
\mathbf{E} \\
\mathbf{E} \\
\mathbf{E}\end{array}$ & $\begin{array}{l}\mathbf{E} \\
\mathbf{E} \\
\mathbf{E} \\
\mathbf{E} \\
\mathbf{E}\end{array}$ & $\begin{array}{c}E \\
D \\
D \\
B^{\prime} \\
D\end{array}$ & $\begin{array}{c}\text { ND } \\
\text { F } \\
\text { F } \\
\text { F } \\
\text { F }\end{array}$ & $\begin{array}{l}\mathbf{F} \\
\mathbf{F} \\
\mathbf{F} \\
\mathbf{F} \\
\mathbf{E}\end{array}$ & $\begin{array}{l}\mathbf{F} \\
\mathbf{F} \\
\mathbf{F} \\
\mathbf{F} \\
\mathbf{F}\end{array}$ & \begin{tabular}{|c|} 
D \\
D \\
D \\
$B^{\prime}$ \\
D
\end{tabular} & $\begin{array}{l}\mathbf{F} \\
\mathbf{F} \\
\mathbf{F} \\
\mathbf{F} \\
\mathbf{F}\end{array}$ & $\begin{array}{c}\text { ND } \\
\text { ND } \\
\text { ND } \\
\text { F } \\
\text { ND }\end{array}$ & $\begin{array}{l}\mathbf{E} \\
\mathbf{E} \\
\mathbf{E} \\
\mathbf{D} \\
\mathbf{E}\end{array}$ & $\begin{array}{c}\text { A } \\
\mathbf{B}^{\prime} \\
\text { A } \\
\mathbf{B}^{\prime} \\
\mathbf{A}\end{array}$ & $\begin{array}{c}\mathbf{B}^{\prime} \\
\mathbf{A} \\
\mathbf{B}^{\prime} \\
\mathbf{A} \\
\mathbf{B}^{\prime}\end{array}$ & $\begin{array}{l}\mathbf{G} \\
\mathbf{G} \\
\mathbf{G} \\
\mathbf{G} \\
\mathbf{G}\end{array}$ & \begin{tabular}{|c|}
$\mathbf{D}$ \\
$\mathbf{D}$ \\
$\mathbf{D}$ \\
$\mathbf{D}$ \\
$\mathbf{B}^{\prime}$
\end{tabular} & $\begin{array}{l}\mathbf{E} \\
\mathbf{E} \\
\mathbf{E} \\
\mathbf{E} \\
\mathbf{E}\end{array}$ & \begin{tabular}{|c|}
$\mathbf{F}$ \\
$\mathbf{N D}$ \\
$\mathbf{F}$ \\
$\mathrm{ND}$ \\
$\mathbf{F}$
\end{tabular} & $\begin{array}{l}\mathbf{E} \\
\mathbf{D} \\
\mathbf{E} \\
\mathbf{E} \\
\mathbf{E}\end{array}$ & $\begin{array}{l}\text { D } \\
\text { D } \\
\text { D } \\
\text { D } \\
\text { D }\end{array}$ & $\begin{array}{l}\mathbf{E} \\
\mathbf{F} \\
\mathbf{E} \\
\mathbf{E} \\
\mathbf{E}\end{array}$ & $\begin{array}{l}\mathbf{G} \\
\mathbf{G} \\
\mathbf{G} \\
\mathbf{G} \\
\mathbf{G}\end{array}$ & $\begin{array}{l}\mathbf{E} \\
\mathbf{E} \\
\mathbf{E} \\
\mathbf{E} \\
\mathbf{E}\end{array}$ & $\begin{array}{l}\mathbf{E} \\
\mathbf{E} \\
\mathbf{E} \\
\mathbf{E} \\
\mathbf{E}\end{array}$ \\
\hline $\begin{array}{l}\text { P- } 6 \\
\text { P- } 5 \\
\text { P- } 4 \\
\text { P- } 3 \\
\text { P- } 2 \\
\text { P- } 1\end{array}$ & $\begin{array}{l}1462-\text { WOM } \\
1461-\text { WOM } \\
1460-W O M \\
1449-W O M \\
1448-W O M \\
1447-W O M\end{array}$ & $\begin{array}{l}\mathrm{B}^{\mathbf{t}} \\
\mathrm{B}^{\prime} \\
\mathrm{D} \\
\mathrm{D} \\
\mathrm{A} \\
\mathrm{D}\end{array}$ & $\begin{array}{l}\mathbf{E} \\
\mathbf{E} \\
\mathbf{E} \\
\mathbf{E} \\
\mathbf{E} \\
\mathbf{E}\end{array}$ & $\begin{array}{l}\text { G } \\
\text { ND } \\
\text { G } \\
\text { ND } \\
\text { F } \\
\text { G }\end{array}$ & $\begin{array}{l}\mathbf{E} \\
\mathbf{E} \\
\mathbf{E} \\
\mathbf{E} \\
\mathbf{F} \\
\mathbf{E}\end{array}$ & $\begin{array}{c}\text { A } \\
\text { A } \\
\text { A } \\
\text { A } \\
\text { B }^{\prime} \\
\\
\text { A }\end{array}$ & $\begin{array}{l}\mathbf{E} \\
\mathbf{E} \\
\mathbf{E} \\
\mathbf{E} \\
\mathbf{E} \\
\mathbf{F}\end{array}$ & $\begin{array}{l}\mathbf{E} \\
\mathbf{E} \\
\mathbf{E} \\
\mathbf{E} \\
\mathbf{E} \\
\mathbf{F}\end{array}$ & $\begin{array}{c}\mathbf{D} \\
\mathbf{E} \\
\mathbf{E} \\
\mathbf{E} \\
\mathbf{B}^{\prime} \\
\mathbf{B}^{\prime}\end{array}$ & $\begin{array}{c}F \\
\text { ND } \\
F \\
F \\
F \\
\text { ND }\end{array}$ & $\begin{array}{c}E \\
E \\
E \\
F \\
\text { ND } \\
E\end{array}$ & $\begin{array}{c}\text { ND } \\
F \\
F \\
F \\
F \\
\text { ND }\end{array}$ & $\begin{array}{c}\text { D } \\
\text { D } \\
\text { D } \\
\text { D } \\
B^{\prime} \\
\text { D }\end{array}$ & $\begin{array}{l}\mathbf{E} \\
\mathbf{F} \\
\mathbf{F} \\
\mathbf{F} \\
\mathbf{D} \\
\mathbf{D}\end{array}$ & $\begin{array}{l}\text { ND } \\
\text { ND } \\
\text { ND } \\
\text { ND } \\
\text { ND } \\
\text { ND }\end{array}$ & $\begin{array}{l}\mathbf{E} \\
\mathbf{E} \\
\mathbf{E} \\
\mathbf{E} \\
\mathbf{D} \\
\mathbf{D}\end{array}$ & \begin{tabular}{|c} 
A \\
A \\
A \\
A \\
D \\
ND
\end{tabular} & $\begin{array}{l}\mathbf{B}^{\prime} \\
\mathbf{B}^{\prime} \\
\mathbf{B}^{\prime} \\
\mathbf{B}^{\prime} \\
\mathbf{A} \\
\mathbf{B}^{\prime}\end{array}$ & $\begin{array}{l}\mathbf{G} \\
\mathbf{G} \\
\mathbf{G} \\
\mathbf{G} \\
\mathbf{G} \\
\mathbf{G}\end{array}$ & $\begin{array}{c}B^{\prime} \\
B^{\prime} \\
B^{\prime} \\
B^{\prime} \\
D \\
B^{t}\end{array}$ & $\begin{array}{l}\mathbf{E} \\
\mathbf{E} \\
\mathbf{E} \\
\mathbf{E} \\
\mathbf{E} \\
\mathbf{E}\end{array}$ & $\mid \begin{array}{c}F \\
F \\
F \\
F \\
\text { ND } \\
\text { ND }\end{array}$ & $\begin{array}{l}\mathbf{E} \\
\mathbf{E} \\
\mathbf{E} \\
\mathbf{E} \\
\mathbf{D} \\
\mathbf{E}\end{array}$ & $\begin{array}{l}\mathrm{D} \\
\mathrm{D} \\
\mathrm{D} \\
\mathrm{D} \\
\mathrm{D} \\
\mathrm{D}\end{array}$ & $\begin{array}{l}\mathbf{E} \\
\mathbf{E} \\
\mathbf{E} \\
\mathbf{E} \\
\mathbf{F} \\
\mathbf{E}\end{array}$ & $\begin{array}{l}\mathbf{G} \\
\mathbf{G} \\
\mathbf{G} \\
\mathbf{G} \\
\mathbf{G} \\
\mathbf{G}\end{array}$ & $\begin{array}{l}\mathbf{E} \\
\mathbf{E} \\
\mathbf{E} \\
\mathbf{E} \\
\mathbf{D} \\
\mathbf{E}\end{array}$ & $\begin{array}{l}\mathbf{E} \\
\mathbf{F} \\
\mathbf{F} \\
\mathbf{F} \\
\mathbf{E} \\
\mathbf{F}\end{array}$ \\
\hline
\end{tabular}


TRAIL CANYON, IDAHO. LOT NO. 1206.

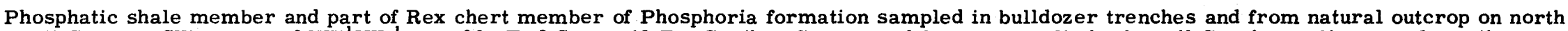

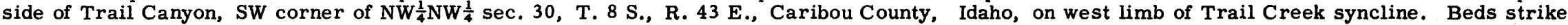

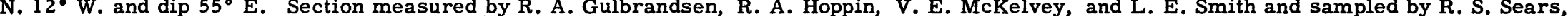

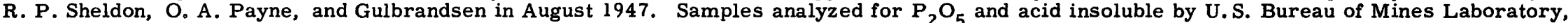
Albany, Oregon, and for other constituents by Trace Elements Section Laboratory, U. ${ }^{2}$. Geological Survey, Washington, D. C.

\begin{tabular}{|c|c|c|c|c|c|c|c|c|c|c|c|}
\hline \multirow{2}{*}{$\begin{array}{c}\text { Bed } \\
\text { no. }\end{array}$} & \multirow[b]{2}{*}{ Rock description } & \multirow{2}{*}{$\begin{array}{c}\text { Sample } \\
\text { no. }\end{array}$} & \multirow{2}{*}{$\begin{array}{c}\text { Thickness } \\
\text { (feet) }\end{array}$} & \multicolumn{6}{|c|}{ Chemical analyses (percent) } & \multirow{2}{*}{$\begin{array}{c}\text { Cumulative } \\
\text { thickness } \\
\text { (feet) }\end{array}$} & \multirow{2}{*}{$\begin{array}{l}\text { Thickness } x \\
\text { percent } \mathrm{P}_{2} \mathrm{O}_{5} \\
\text { (cumulative) }\end{array}$} \\
\hline & & & & $\mathrm{P}_{2} \mathrm{O}_{5}$ & $\mathrm{Al}_{2} \mathrm{O}_{3}$ & $\mathrm{Fe}_{2} \mathrm{O}_{3}$ & $\mathbf{F}$ & $\begin{array}{l}\text { Loss on } \\
\text { ignition }\end{array}$ & $\begin{array}{c}\text { Acid } \\
\text { insoluble }\end{array}$ & & \\
\hline \multicolumn{12}{|c|}{ Rex member of Phosphoria formation } \\
\hline $\begin{array}{l}R-39 \\
R-38 \\
R-37 \\
R-36 \\
R-35\end{array}$ & $\begin{array}{l}\text { Chert } \\
\text { Chert } \\
\text { Chert, silty } \\
\text { Chert } \\
\text { Chert, silty }\end{array}$ & $\begin{array}{l}\text { RAH- } 241-47 \\
\text { RAH- } 240-47 \\
\text { RAH- } 239-47 \\
\text { RAH- } 238-47 \\
\text { RAH- } 237-47\end{array}$ & $\begin{array}{r}8.4 \\
10.8 \\
8.9 \\
9.7 \\
7.9\end{array}$ & $\begin{array}{l}0.4 \\
0.6 \\
0.5 \\
0.6 \\
0.6\end{array}$ & $\begin{array}{l}-- \\
-- \\
--\end{array}$ & $\begin{array}{l}-- \\
-- \\
-- \\
--\end{array}$ & $\begin{array}{l}-- \\
-- \\
-- \\
--\end{array}$ & $\begin{array}{l}-- \\
-- \\
-- \\
--\end{array}$ & $\begin{array}{l}88.4 \\
87.5 \\
88.4 \\
87.9 \\
88.8\end{array}$ & $\begin{array}{l}8.4 \\
19.2 \\
28.1 \\
37.8 \\
45.7\end{array}$ & $\begin{array}{r}4.20 \\
10.68 \\
15.13 \\
20.95 \\
25.69\end{array}$ \\
\hline $\begin{array}{l}R-34 \\
R-33 \\
R-32 \\
R-31 \\
R-30\end{array}$ & $\begin{array}{l}\text { Mudstone } \\
\text { Mudstone } \\
\text { Mudstone } \\
\text { Mudstone } \\
\text { Chert }\end{array}$ & $\begin{array}{l}\text { RAH- } 236-47 \\
\text { RAH- } 235-47 \\
\text { RAH- } 234-47 \\
\text { RAH- } 233-47 \\
\text { RAH- } 232-47\end{array}$ & $\begin{array}{r}6.8 \\
8.2 \\
10.0 \\
6.7 \\
2.9\end{array}$ & $\begin{array}{l}0.4 \\
0.5 \\
0.5 \\
0.5 \\
1.2\end{array}$ & $\begin{array}{l}-- \\
-- \\
-- \\
--\end{array}$ & $\begin{array}{l}-- \\
-- \\
-- \\
--\end{array}$ & $\begin{array}{l}-- \\
-- \\
-- \\
--\end{array}$ & $\begin{array}{l}-- \\
-- \\
-- \\
--\end{array}$ & $\begin{array}{l}88.2 \\
87.0 \\
87.8 \\
85.5 \\
86.4\end{array}$ & $\begin{array}{l}52.5 \\
60.7 \\
70.7 \\
77.4 \\
80.3\end{array}$ & $\begin{array}{l}28.41 \\
32.51 \\
37.51 \\
40.86 \\
44.34\end{array}$ \\
\hline $\begin{array}{l}R-29 \\
R-28 \\
R-27 \\
R-26 \\
R-25\end{array}$ & $\begin{array}{l}\text { Chert } \\
\text { Chert } \\
\text { Chert } \\
\text { Chert } \\
\text { Chert }\end{array}$ & $\begin{array}{l}\text { RAH- } 231-47 \\
\text { RAH- } 230-47 \\
\text { RAH- } 229-47 \\
\text { RAH- } 228-47 \\
\text { RAH- } 227-47\end{array}$ & $\begin{array}{r}3.1 \\
7.4 \\
7.3 \\
12.4 \\
6.0\end{array}$ & $\begin{array}{l}1.1 \\
0.1 \\
0.4 \\
0.7 \\
0.5\end{array}$ & $\begin{array}{l}-- \\
-- \\
-- \\
-- \\
--\end{array}$ & $\begin{array}{l}-- \\
-- \\
-- \\
--\end{array}$ & $\begin{array}{l}-- \\
-- \\
--\end{array}$ & $\begin{array}{l}-- \\
-- \\
-- \\
--\end{array}$ & $\begin{array}{l}86.1 \\
90.8 \\
94.4 \\
94.5 \\
94.9\end{array}$ & $\begin{array}{r}83.4 \\
90.8 \\
98.1 \\
110.5 \\
116.5\end{array}$ & $\begin{array}{l}47.75 \\
48.49 \\
51.41 \\
60.09 \\
63.09\end{array}$ \\
\hline $\begin{array}{l}R-24 \\
R-23 \\
R-22 \\
R-21 \\
R-20\end{array}$ & $\begin{array}{l}\text { Chert } \\
\text { Chert } \\
\text { Chert } \\
\text { Chert } \\
\text { Chert }\end{array}$ & $\begin{array}{l}\text { RAH- } 226-47 \\
\text { RAH- } 225-47 \\
\text { RAH }-224-47 \\
\text { RAH- } 223-47 \\
\text { RAH- } 222-47\end{array}$ & $\begin{array}{r}5.6 \\
4.7 \\
6.7 \\
1.6 \\
10.5\end{array}$ & $\begin{array}{l}0.2 \\
0.3 \\
0.4 \\
0.7 \\
0.6\end{array}$ & $\begin{array}{l}-- \\
-- \\
-- \\
--\end{array}$ & $\begin{array}{l}-- \\
-- \\
-- \\
--\end{array}$ & $\begin{array}{l}-- \\
-- \\
--\end{array}$ & $\begin{array}{l}-- \\
-- \\
-- \\
--\end{array}$ & $\begin{array}{l}94.1 \\
93.7 \\
94.8 \\
94.7 \\
92.9\end{array}$ & $\begin{array}{l}122.1 \\
126.8 \\
133.5 \\
135.1 \\
145.6\end{array}$ & $\begin{array}{l}64.21 \\
65.62 \\
68.30 \\
69.42 \\
75.72\end{array}$ \\
\hline $\begin{array}{l}R-19 \\
R-18 \\
R-17 \\
R-16 \\
R-15\end{array}$ & $\begin{array}{l}\text { Chert } \\
\text { Chert } \\
\text { Chert and limestone } \\
\text { Chert and limestone } \\
\text { Chert and limestone; fos. col. no. } \\
47-\mathrm{HW}-152^{1}\end{array}$ & $\begin{array}{l}\text { RAH- } 221-47 \\
\text { RAH- 220-47 } \\
\text { RAH- 219-47 } \\
\text { RAH- 218-47 } \\
\text { RAH- 218-47 }\end{array}$ & $\begin{array}{r}8.3 \\
10.2 \\
3.6 \\
8.7 \\
3.8\end{array}$ & $\begin{array}{l}0.2 \\
0.2 \\
0.3 \\
0.6 \\
0.6\end{array}$ & $\begin{array}{l}-- \\
-- \\
-- \\
--\end{array}$ & $\begin{array}{l}-- \\
-- \\
-- \\
--\end{array}$ & $\begin{array}{l}-- \\
-- \\
-- \\
--\end{array}$ & $\begin{array}{l}-- \\
-- \\
-- \\
--\end{array}$ & $\begin{array}{l}86.6 \\
89.4 \\
82.6 \\
81.5 \\
82.6\end{array}$ & $\begin{array}{l}153.9 \\
164.1 \\
167.7 \\
176.4 \\
180.2\end{array}$ & $\begin{array}{l}77.38 \\
79.42 \\
80.50 \\
85.72 \\
88.00\end{array}$ \\
\hline
\end{tabular}

Preceding section measured along road (beds $R-26$ to $R-39$ ) and in cut 50 feet above road (beds $R-15$ to $R-25$ ); following section (beds $R-1$ to $R-14$ ) measured in trench 200 feet above road. Contact between beds $R-25$ and $R-26$ (black chert and pinkish white chert) is exposed at road and lower cut; beds below $R-15$ are concealed along road and beds $R-15$ to $R-25$ are largely concealed at upper trench level.

Thickness of beds $R-15$ to $R-25$ is 69.7 feet, but computed thickness of same sequence at upper trench is 59 feet. The larger (measured) figure is used in computing total thickness of Rex member.

${ }^{1}$ Fossil collection made by H. Wedow, Paleontology and Stratigraphy Branch, U. S. Geological Survey. 


\begin{tabular}{|c|c|c|c|c|c|c|c|c|c|c|c|}
\hline \multirow{2}{*}{$\begin{array}{c}\text { Bed } \\
\text { no. }\end{array}$} & \multirow[b]{2}{*}{ Rock description } & \multirow{2}{*}{$\begin{array}{l}\text { Sample } \\
\text { no. }\end{array}$} & \multirow{2}{*}{$\begin{array}{l}\text { Thickness } \\
\text { (feet) }\end{array}$} & \multicolumn{6}{|c|}{ Chemical analyses (percent) } & \multirow{2}{*}{$\begin{array}{c}\text { Cumulative } \\
\text { thickness } \\
\text { (feet) }\end{array}$} & \multirow{2}{*}{$\begin{array}{l}\text { Thickness } x \\
\text { percent } \mathrm{P}_{2} \mathrm{O}_{5} \\
\text { (cumulative) }\end{array}$} \\
\hline & & & & $\mathrm{P}_{2} \mathrm{O}_{5}$ & $\mathrm{Al}_{2} \mathrm{O}_{3}$ & $\mathrm{Fe}_{2} \mathrm{O}_{3}$ & $\mathbf{F}$ & $\begin{array}{l}\text { Loss on } \\
\text { ignition }\end{array}$ & $\begin{array}{l}\text { Acid } \\
\text { insoluble }\end{array}$ & & \\
\hline$R-14$ & Limestone, cherty & RAH - 213-47 & 0.9 & 0.4 & -- & -- & -- & - & 42.9 & 181.1 & 88.36 \\
\hline$R-13$ & Chert & RAH- $212-47$ & 3.1 & 0.7 & -- & -- & -- & -- & 81.9 & 184.2 & 90.53 \\
\hline $\mathrm{R}-12$ & Limestone, locally cherty & RAH $-211-47$ & 0.7 & 0.3 & -- & -- & -- & -- & 28.5 & 184.9 & 90.74 \\
\hline$R-11$ & limestone concretions & RAH- $210-47$ & 8.1 & 0.6 & -- & -- & -- & -- & 73.3 & 193.0 & 95.60 \\
\hline$R-10$ & Chert, contains limestone & & & & & & & & & & \\
\hline & concretions and lenses & RAH- $207-48$ & 0.4 & 0.6 & $=-$ & $\ldots$ & - & 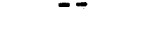 & 86.2 & 199. & 99.44 \\
\hline $\begin{array}{ll}R- & 9 \\
R- & 8\end{array}$ & Limestone, locally cherty & RAH- 208-47 & 0.8 & 0.2 & -- & -- & -- & -- & 31.0 & 200.2 & 99.60 \\
\hline & lenses & RAH- 207-47 & 5.7 & 0.4 & - & -- & -- & -- & 77.7 & 205.9 & 101.88 \\
\hline $\begin{array}{ll}R- & 7 \\
R- & 6\end{array}$ & $\begin{array}{l}\text { Limestone, argillace ous } \\
\text { Chert, contains thin limestone }\end{array}$ & RAH- 206-47 & 1.3 & 0.6 & -- & -- & -- & -- & 30.5 & 207.2 & 102.66 \\
\hline & lenses & RAH- 205-47 & 3.3 & 0.7 & -- & -- & -- & -- & 88. 7 & 210.5 & 104.97 \\
\hline$R-5$ & $\begin{array}{l}\text { Limestone, contains cherty } \\
\text { limestone beds }\end{array}$ & $\mathrm{RAH}-204-47$ & 2.0 & 0.7 & -- & -- & -- & -- & 45.5 & 212.5 & 106.37 \\
\hline R- 4 & Chert & RAH- 203-47 & 5.6 & 0.8 & -- & -- & -- & -- & 85.8 & 218.1 & 110.85 \\
\hline$R-3$ & Limestone, argillaceous & RAH- $202-47$ & 3.3 & 0.6 & -- & -- & -- & -- & 32.9 & 221.4 & 112.83 \\
\hline$R-2$ & Chert & RAH- $201-47$ & 4.0 & 0.8 & -- & -- & -- & -- & 90.5 & 225.4 & 116.03 \\
\hline$R-1$ & Chert, contains mudstone layers & RAH- $200-47$ & 2.7 & 0.7 & -- & -- & -- & -- & 87.8 & 228.1 & 117.92 \\
\hline
\end{tabular}

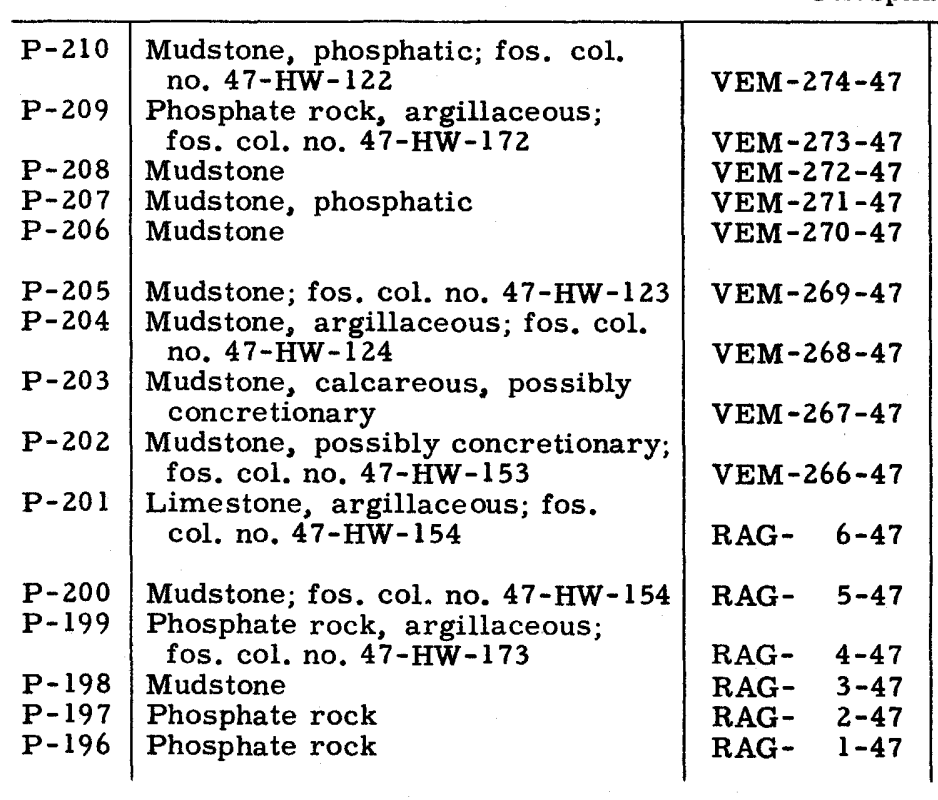

\begin{tabular}{|l|r|r|r|l|}
\hline 0.3 & 8.0 & -- & - & -- \\
0.4 & 26.6 & -- & -- & -- \\
0.9 & 1.4 & -- & -- & -- \\
0.7 & 8.8 & -- & -- & -- \\
0.9 & 1.8 & -- & -- & -- \\
0.7 & 2.2 & -- & -- & -- \\
2.9 & 0.2 & -- & -- & -- \\
0.7 & 1.1 & -- & -- & -- \\
1.4 & 1.3 & -- & -- & -- \\
1.24 & 2.4 & -- & -- & -- \\
1.65 & 3.2 & -- & -- & -- \\
0.73 & 23.1 & -- & -- & 2.14 \\
0.71 & 6.9 & -- & -- & 1.09 \\
1.15 & 28.6 & 1.6 & 0.80 & 2.75 \\
0.68 & 28.0 & 2.3 & 0.98 & 2.90 \\
& & & &
\end{tabular}

--
--
--
--
--
--
--
--
--
--
--
--
9.64
8.86

68.1
21.5
77.5
57.9
75.1
70.6
53.9
67.0
78.4
39.8
72.6
25.5
56.7
9.4
12.9

0.3
0.7
1.6
2.3
3.2
3.9
6.8
7.5
8.9
10.14
11.79
12.52
13.23
14.38
15.06

2.40

13.04 14.30 20.46 22.08 23.62 24.20

24.97

26. 79 29.67 35.11 51.28 56. 19 89.0 108.13 


\begin{tabular}{|c|c|}
\hline$P-195$ & Phospha \\
\hline$P-194$ & Phosphate rock \\
\hline$P-193$ & $\begin{array}{l}\text { Phosphate rock, contains } \\
\text { phosphatic limestone concretion } \\
0.2 \text { foot thick }\end{array}$ \\
\hline$P-192$ & Mudstone \\
\hline$P-191$ & Phosphate rock \\
\hline$P-190$ & Limestone \\
\hline$P-189$ & Mudstone \\
\hline$P-188$ & Phosphate rock \\
\hline-- & $\begin{array}{l}\text { Phosphate rock, calcareous, } \\
\text { concretion in bed } P-188\end{array}$ \\
\hline$P-187$ & $\begin{array}{l}\text { Phosphate rock and phosphatic } \\
\text { mudstone; fos. col. no. } 47-\mathrm{HW}-155\end{array}$ \\
\hline$P-186$ & $\begin{array}{l}\text { Phosphate rock, calcareous, } \\
\text { contains phosphatic mudstone } \\
\text { concretions; fos. col. no. } \\
47-\mathrm{HW}-156\end{array}$ \\
\hline$P-185$ & Phosphate rock, argillaceous \\
\hline$P-184$ & Phosphate rock, calcareous \\
\hline-- & $\begin{array}{l}\text { Limestone concretion; fos. col. } \\
\text { no. } 47-\mathrm{HW}-157\end{array}$ \\
\hline$P-183$ & $\begin{array}{l}\text { Mudstone, calcareous, phosphatic; } \\
\text { fos. col. no. } 47-\mathrm{HW}-158\end{array}$ \\
\hline $\begin{array}{l}P-182 \\
P-181 \\
P-180 \\
P-179 \\
P-178\end{array}$ & $\begin{array}{l}\text { Mudstone; fos. col. no. } 47-\mathrm{HW}-159 \\
\text { Mudstone; fos. col. no. } 47-\mathrm{HW}-160 \\
\text { Mudstone; fos. col. no. } 47-\mathrm{HW}-161 \\
\text { Mudstone; fos. col. no. } 47-\mathrm{HW}-162 \\
\text { Phosphate rock, argillaceous }\end{array}$ \\
\hline$P-177$ & Mudstone, phosphatic \\
\hline $\mathrm{P}-176$ & Phosphate rock \\
\hline $\mathrm{P}-175$ & $\begin{array}{l}\text { Phosphate rock, argillaceous, } \\
\text { and calcareous mudstone }\end{array}$ \\
\hline$P-174$ & $\begin{array}{l}\text { Phosphate rock, calcareous, } \\
\text { argillaceous; fos, col. nos. } \\
47-\mathrm{HW}-163 \text { and } 47-\mathrm{HW}-164\end{array}$ \\
\hline P-173 & $\begin{array}{l}\text { Phosphate rock, calcareous; } \\
\text { fos. col. no. } 47-\mathrm{HW}-165\end{array}$ \\
\hline P-17 & $\begin{array}{l}\text { Limestone, phosphatic, } \\
\text { argillaceous; fos. col. no. } \\
\text { 47-HW-166 }\end{array}$ \\
\hline$P-171$ & $\begin{array}{l}\text { Phosphate rock, calcareous, } \\
\text { argillaceous; fos. col. no. } \\
\text { 47-HW-167 }\end{array}$ \\
\hline$P-17$ & $\begin{array}{l}\text { Phosphate rock, calcareous, } \\
\text { argillaceous; fos. col. no. } \\
47-\mathrm{HW}-168\end{array}$ \\
\hline
\end{tabular}

\begin{tabular}{|c|c|c|c|c|c|c|c|c|c|}
\hline $\begin{array}{l}\text { VEM }-265-47 \\
\text { VEM }-264-47\end{array}$ & $\begin{array}{l}0.6 \\
0.8\end{array}$ & $\begin{array}{l}36.7 \\
31.6\end{array}$ & $\begin{array}{l}0.7 \\
1.9\end{array}$ & $\begin{array}{l}0.58 \\
1.3\end{array}$ & $\begin{array}{l}3.65 \\
3.04\end{array}$ & $\begin{array}{l}4.18 \\
5.22\end{array}$ & $\begin{array}{r}3.2 \\
11.7\end{array}$ & $\begin{array}{l}15.66 \\
16.46\end{array}$ & $\begin{array}{l}130.15 \\
155.43\end{array}$ \\
\hline $\begin{array}{l}\text { VEM }-263-47 \\
\text { VEM }-262-47 \\
\text { VEM }-259-47\end{array}$ & $\begin{array}{l}0.6 \\
0.3 \\
1.0\end{array}$ & $\begin{array}{r}33.9 \\
2.7 \\
30.6\end{array}$ & $\begin{array}{l}1.1 \\
9.8 \\
1.5\end{array}$ & $\begin{array}{l}0.80 \\
3.7 \\
0.97\end{array}$ & $\begin{array}{l}3.23 \\
0.31 \\
2.97\end{array}$ & $\begin{array}{r}5.22 \\
10.14 \\
5.02\end{array}$ & $\begin{array}{r}6.7 \\
70.2 \\
14.6\end{array}$ & $\begin{array}{l}17.06 \\
17.36 \\
18.36\end{array}$ & $\begin{array}{l}175.86 \\
176.67 \\
207.27\end{array}$ \\
\hline $\begin{array}{l}\text { VEM }-258-47 \\
\text { VEM }-257-47 \\
\text { VEM }-256-47\end{array}$ & $\begin{array}{l}1.0 \\
0.4 \\
0.6\end{array}$ & $\begin{array}{r}2.7 \\
0.3 \\
34.0\end{array}$ & $\begin{array}{l}1.4 \\
1.4 \\
0.8\end{array}$ & $\begin{array}{l}0.80 \\
0.68 \\
0.61\end{array}$ & $\begin{array}{l}-- \\
-- \\
3.27\end{array}$ & $\begin{array}{r}35.88 \\
9.58 \\
6.18\end{array}$ & $\begin{array}{r}12.0 \\
83.0 \\
5.7\end{array}$ & $\begin{array}{l}19.36 \\
19.76 \\
20.36\end{array}$ & $\begin{array}{l}209.97 \\
210.09 \\
230.49\end{array}$ \\
\hline VEM $-255-47$ & $(0.0-0.4)$ & 23.5 & 0.8 & 0.45 & 2.26 & 16.14 & 7.3 & -- & -- \\
\hline VEM-254-47 & 0.3 & 16.4 & 6.0 & 2.0 & 1.61 & 10.12 & 36.3 & 20.66 & 235.41 \\
\hline $\begin{array}{l}\text { VEM-253-47 } \\
\text { VEM-252-47 } \\
\text { VEM-251-47 }\end{array}$ & $\begin{array}{l}0.6 \\
0.4 \\
0.8\end{array}$ & $\begin{array}{l}20.9 \\
20.0 \\
23.5\end{array}$ & $\begin{array}{l}1.8 \\
3.8 \\
0.8\end{array}$ & $\begin{array}{l}0.66 \\
1.3 \\
0.91\end{array}$ & $\begin{array}{l}2.06 \\
2.06 \\
2.22\end{array}$ & $\begin{array}{l}19.68 \\
12.16 \\
15.96\end{array}$ & $\begin{array}{r}9.3 \\
25.5 \\
7.3\end{array}$ & $\begin{array}{l}21.26 \\
21.66 \\
22.46\end{array}$ & $\begin{array}{l}247.95 \\
255.95 \\
274.75\end{array}$ \\
\hline VEM-250-47 & $(0.0-0.7)$ & 1.6 & -- & -- & -- & -- & 11.4 & - & -- \\
\hline VEM-249-47 & 1.2 & 9.1 & -- & -- & -- & -- & 42.8 & 23.66 & 285.67 \\
\hline $\begin{array}{l}\text { VEM-248-47 } \\
\text { VEM }-247-47 \\
\text { VEM }-246-47 \\
\text { VEM-245-47 } \\
\text { VEM }-244-47\end{array}$ & $\begin{array}{l}0.5 \\
0.8 \\
1.1 \\
1.0 \\
0.6\end{array}$ & $\begin{array}{r}3.1 \\
3.9 \\
3.3 \\
3.5 \\
16.2\end{array}$ & $\begin{array}{l}-- \\
-- \\
-- \\
--\end{array}$ & $\begin{array}{l}-- \\
-- \\
-- \\
--\end{array}$ & $\begin{array}{l}-- \\
-- \\
-- \\
--\end{array}$ & $\begin{array}{l}-- \\
-- \\
-- \\
--\end{array}$ & $\begin{array}{l}50.9 \\
61.4 \\
63.1 \\
65.3 \\
31.6\end{array}$ & $\begin{array}{l}24.16 \\
24.96 \\
26.06 \\
27.06 \\
27.66\end{array}$ & $\begin{array}{l}287.22 \\
290.34 \\
293.97 \\
297.47 \\
307.19\end{array}$ \\
\hline $\begin{array}{l}\text { VEM }-243-47 \\
\text { VEM }-242-47\end{array}$ & $\begin{array}{l}1.0 \\
0.5\end{array}$ & $\begin{array}{l}15.7 \\
33.6\end{array}$ & -- & -- & $\overline{--}$ & $\overline{-}$ & $\begin{array}{r}41.8 \\
3.8\end{array}$ & $\begin{array}{l}28.66 \\
29.16\end{array}$ & $\begin{array}{l}322.89 \\
339.69\end{array}$ \\
\hline VEM-241-47 & 0.8 & 25.5 & -- & -- & -- & - & 14.8 & 29.96 & 360.09 \\
\hline VEM $-240-47$ & 0.8 & 18.7 & -- & -- & -- & -- & 23.1 & 30.76 & 375.05 \\
\hline VEM-239-47 & 0.7 & 17.2 & -- & -- & -- & -- & 14.2 & 31.46 & 387.09 \\
\hline VEM $-238-47$ & 0.4 & 12.7 & -- & -- & -- & - & 23.4 & 31.86 & 392.17 \\
\hline VEM $-261-47$ & 0.6 & 16.6 & -- & -- & -- & -- & 20.1 & 32.46 & 402.13 \\
\hline LES- $356-47$ & 0.4 & 16.3 & -- & -- & -- & -- & 21.4 & 32.86 & 408.65 \\
\hline
\end{tabular}




\begin{tabular}{|c|c|c|c|c|c|c|c|c|c|c|c|}
\hline \multirow[b]{2}{*}{$\begin{array}{c}\text { Bed } \\
\text { no. }\end{array}$} & \multirow[b]{2}{*}{ Rock description } & \multirow[b]{2}{*}{$\begin{array}{l}\text { Sample } \\
\text { no. }\end{array}$} & \multirow[b]{2}{*}{$\begin{array}{c}\text { Thickness } \\
\text { (feet) }\end{array}$} & \multicolumn{6}{|c|}{ Chemical analyses (percent) } & \multirow{2}{*}{$\begin{array}{l}\text { Cumulative } \\
\text { thickness } \\
\text { (feet) }\end{array}$} & \multirow{2}{*}{$\begin{array}{l}\text { Thickness } x \\
\text { percent } P_{2} O_{5} \\
\text { (cumulative) }\end{array}$} \\
\hline & & & & $\mathrm{P}_{2} \mathrm{O}_{5}$ & $\mathrm{Al}_{2} \mathrm{O}_{3}$ & $\mathrm{Fe}_{2} \mathrm{O}_{3}$ & $F$ & $\begin{array}{l}\text { Loss on } \\
\text { ignition }\end{array}$ & $\begin{array}{c}\text { Acid } \\
\text { insoluble }\end{array}$ & & \\
\hline-- & Limestone & VEM-260-47 & $(0.0-0.3)$ & 6.0 & -- & -- & -- & -- & 7.6 & -- & -- \\
\hline$P-169$ & $\begin{array}{l}\text { VEM-260 represents two concreti } \\
\text { Phosphate rock, calcareous, } \\
\text { argillaceous, contains } \\
\text { calcareous concretions; } \\
\text { fos, col }\end{array}$ & ons of $0.0-0.3=$ & nd $0.0-0.2$ & oot thi & ckness & in beds & -171 & nd $P-170$ & respectivel & 33.61 & 425.45 \\
\hline \multirow{3}{*}{$\begin{array}{l}P-168 \\
-- \\
P-167\end{array}$} & $\begin{array}{l}\text { Phosphate rock, calcareous; } \\
\text { fos. col. no. } 47-\mathrm{HW}-170\end{array}$ & LES- $349-47$ & 0.6 & 16.6 & -- & -- & -- & -- & 19.5 & 34.21 & 435.41 \\
\hline & $\begin{array}{l}\text { Limestone concretion in bed } \\
\text { P-168 }\end{array}$ & LES- $350-47$ & $(0.45)$ & 1.3 & - & - & -- & $\cdots$ & 1.9 & - & -- \\
\hline & $\begin{array}{l}\text { Limestone, phosphatic, } \\
\text { argillaceous; fos. col. no. } \\
47-\mathrm{HW}-171\end{array}$ & LES- $348-47$ & 0.4 & 10.8 & -- & -- & -- & -- & 26.0 & 34.61 & 439.73 \\
\hline \multirow[t]{2}{*}{$\begin{array}{l}P-166 \\
P-165\end{array}$} & $\begin{array}{l}\text { Limestone, argillaceous, } \\
\text { phosphatic } \\
\text { Limestone, phosphatic, }\end{array}$ & LES- 347-47 & 0.55 & 8.9 & -- & -- & -- & $\cdots$ & 29.1 & 35.16 & 444.63 \\
\hline & $\begin{array}{l}\text { Limestone, phosphatic, } \\
\text { argillaceous; fos. col. no. } \\
47-\text { HW-88 } \\
\text { Phosphate rock, calcareous } \\
\text { Phosphate rock, calcareous }\end{array}$ & $\begin{array}{l}\text { LES- } 346-47 \\
\text { LES- } 345-47 \\
\text { LES- } 344-47\end{array}$ & $\begin{array}{l}0.75 \\
0.55 \\
0.6\end{array}$ & $\begin{array}{l}15.2 \\
18.2 \\
18.0\end{array}$ & $\begin{array}{l}-- \\
--\end{array}$ & $\begin{array}{l}-- \\
--\end{array}$ & $\begin{array}{l}-- \\
--\end{array}$ & $\begin{array}{ll}-- \\
--\end{array}$ & $\begin{array}{l}19.9 \\
13.2 \\
19.1\end{array}$ & $\begin{array}{l}35.91 \\
36.46 \\
37.06\end{array}$ & $\begin{array}{l}456.03 \\
466.03 \\
476.83\end{array}$ \\
\hline $\begin{array}{l}P-164 \\
P-163 \\
P-162\end{array}$ & $\begin{array}{l}\text { Phosphate rock, calcareous, } \\
\text { argillaceous }\end{array}$ & LES- $343-47$ & 0.6 & 15.5 & -- & -- & -- & -- & 29.8 & 37.66 & 486.13 \\
\hline \multirow{2}{*}{$\begin{array}{l}P-161 \\
P-160\end{array}$} & $\begin{array}{l}\text { Phosphate rock, calcareous, } \\
\text { argillaceous }\end{array}$ & LES- $342-47$ & 0.65 & 15.3 & -- & $=$ & -- & -- & 22.6 & 38.31 & 496.08 \\
\hline & $\begin{array}{l}\text { Phosphate rock, calcareous, } \\
\text { argillaceous }\end{array}$ & LES- 341-47 & 0.7 & 17.8 & -- & -- & -- & -- & 20.3 & 39.01 & 508.54 \\
\hline$P-159$ & $\begin{array}{l}\text { Phosphate rock, calcareous, } \\
\text { argillaceous }\end{array}$ & LES- $340-47$ & 0.6 & 18.7 & -- & -- & -- & -- & 22.7 & 39.61 & 519.76 \\
\hline \multirow{2}{*}{$\begin{array}{l}P-158 \\
P-157\end{array}$} & $\begin{array}{l}\text { Phosphate rock, calcareous, } \\
\text { argillaceous }\end{array}$ & LES- $339-47^{*}$ & 0.5 & 18.8 & -- & - & -- & -- & 25.9 & 40.11 & 529.16 \\
\hline & $\begin{array}{l}\text { Phosphate rock, calcareous, } \\
\text { argillaceous, contains limestone } \\
\text { concretions }\end{array}$ & LES- 338-47 & 0.55 & 14.8 & -- & -- & -- & -- & 21.5 & 40.66 & 537.30 \\
\hline $\begin{array}{l}P-156 \\
P-155 \\
P-154 \\
P-153 \\
P-152\end{array}$ & $\begin{array}{l}\text { Phosphate rock, calcareous, } \\
\text { argillaceous } \\
\text { Phosphate rock, argillaceous } \\
\text { Mudstone, phosphatic } \\
\text { Mudstone, phosphatic } \\
\text { Mudstone, phosphatic }\end{array}$ & $\begin{array}{l}\text { LES- } 337-47 \\
\text { LES- } 336-47 \\
\text { LES- } 335-47 \\
\text { LES- } 334-47 \\
\text { LES- } 333-47\end{array}$ & $\begin{array}{l}0.6 \\
0.55 \\
0.6 \\
0.6 \\
0.5\end{array}$ & $\begin{array}{l}16.9 \\
16.4 \\
14.7 \\
15.4 \\
12.8\end{array}$ & $\begin{array}{l}-- \\
-- \\
-- \\
--\end{array}$ & $\begin{array}{l}- \\
- \\
- \\
-- \\
-\end{array}$ & $\begin{array}{l}-- \\
=- \\
-- \\
--\end{array}$ & $\begin{array}{l}-- \\
-- \\
-- \\
--\end{array}$ & $\begin{array}{l}27.9 \\
31.0 \\
39.6 \\
40.1 \\
44.1\end{array}$ & $\begin{array}{l}41.26 \\
41.81 \\
42.41 \\
43.01 \\
43.51\end{array}$ & $\begin{array}{l}547.44 \\
556.46 \\
565.28 \\
574.52 \\
580.92\end{array}$ \\
\hline $\begin{array}{l}P-151 \\
P-150 \\
P-149\end{array}$ & $\begin{array}{l}\text { Mudstone, phosphatic } \\
\text { Limestone, argillaceous } \\
\text { Limestone; fos. col. no. }\end{array}$ & $\begin{array}{l}\text { LES- } 332-47 \\
\text { LES- } 331-47\end{array}$ & $\begin{array}{l}0.6 \\
0.65\end{array}$ & $\begin{array}{r}11.1 \\
4.9\end{array}$ & -- & -- & $=$ & $\therefore$ & $\begin{array}{l}48.4 \\
39.2\end{array}$ & $\begin{array}{l}44.11 \\
44.76\end{array}$ & $\begin{array}{l}587.58 \\
590.77\end{array}$ \\
\hline & $47-\mathrm{HW}-87$ & LES- $330-47$ & 1.3 & 0.9 & -- & -- & -- & -- & 29.6 & 46.06 & 591.94 \\
\hline
\end{tabular}




\begin{tabular}{|c|c|}
\hline $\begin{array}{l}P-148 \\
P-147 \\
P-146 \\
P=145 \\
P-144\end{array}$ & $\begin{array}{l}\text { Mudstone, calcareous } \\
\text { Mudstone, phosphatic } \\
\text { Mudstone, phosphatic } \\
\text { Phosphate rock, argillaceous } \\
\text { Mudstone }\end{array}$ \\
\hline $\begin{array}{l}P-143 \\
P-142 \\
P-141 \\
P-140 \\
P-139\end{array}$ & $\begin{array}{l}\text { Mudstone } \\
\text { Mudstone; fos. col. no. } 47-\mathrm{HW}-86 \\
\text { Mudstone } \\
\text { Mudstone } \\
\text { Mudstone, phosphatic }\end{array}$ \\
\hline $\begin{array}{l}P-138 \\
P-137 \\
P-136 \\
P-135 \\
P-134\end{array}$ & $\begin{array}{l}\text { Mudstone } \\
\text { Phosphate rock, argillaceous } \\
\text { Mudstone } \\
\text { Mudstone } \\
\text { Mudstone, phosphatic }\end{array}$ \\
\hline $\begin{array}{l}P-133 \\
P-132 \\
P-131 \\
P-130 \\
P=129\end{array}$ & $\begin{array}{l}\text { Mudstone, phosphatic } \\
\text { Mudstone } \\
\text { Mudstone; fos. col. no. } 47-\mathrm{HW}-85 \\
\text { Mudstone } \\
\text { Mudstone }\end{array}$ \\
\hline $\begin{array}{l}P-128 \\
P-127 \\
P-126 \\
P-125 \\
P-124\end{array}$ & $\begin{array}{l}\text { Mudstone } \\
\text { Mudstone and phosphate rock } \\
\text { Mudstone } \\
\text { Mudstone, phosphatic } \\
\text { Mudstone; fos, col. no. } 47-\mathrm{HW}-84\end{array}$ \\
\hline $\begin{array}{l}P-123 \\
P-122\end{array}$ & $\begin{array}{l}\text { Mudstone } \\
\text { Mudstone, phosphatic and } \\
\text { phosphate rock }\end{array}$ \\
\hline $\begin{array}{l}P-121 \\
P-120 \\
P-119\end{array}$ & $\begin{array}{l}\text { Mudstone } \\
\text { Mudstone, phosphatic } \\
\text { Phosphate rock, argillaceous }\end{array}$ \\
\hline $\begin{array}{l}P-118 \\
P-117 \\
P-116 \\
P-115 \\
P-114\end{array}$ & $\begin{array}{l}\text { Mudstone } \\
\text { Mudstone } \\
\text { Limestone, argillaceous } \\
\text { Mudstone } \\
\text { Phosphate rock }\end{array}$ \\
\hline $\begin{array}{l}P-113 \\
P-112 \\
P=111 \\
P-110 \\
P-109\end{array}$ & $\begin{array}{l}\text { Mudstone } \\
\text { Mudstone } \\
\text { Limestone, argillaceous } \\
\text { Mudstone } \\
\text { Phosphate rock, argillaceous }\end{array}$ \\
\hline $\begin{array}{l}P-108 \\
P-107 \\
P-106 \\
P-105\end{array}$ & $\begin{array}{l}\text { Mudstone } \\
\text { Mudstone, phosphatic } \\
\text { Mudstone } \\
\text { Mudstone }\end{array}$ \\
\hline
\end{tabular}

VEM-237-47
VEM-236-47
VEM-235-47
VEM-234-47
VEM-233-47

VEM-232-47
VEM-231-47
VEM-230-47
VEM-229-47
VEM-228-47
VEM-227-47
VEM-226-47
VEM-225-47
VEM-224-47
VEM-223-47
VEM-222-47
VEM-221-47
VEM-220-47
VEM 219-47
VEM-218-47
VEM-217-47
VEM-216-47
VEM-215-47
VEM-214-47
VEM-213-47
VEM-212-47
VEM-194-47
VEM-197-47
VEM-211-47
VEM-210-47
VEM-209-47
VEM-208-47
VEM-198-47
VEM-207-47
VEM-206-47
VEM-205-47
VEM-204-47
VEM-203-47
VEM-202-47
VEM-201-47
VEM-200-47
VEM-199-47
VEM

\begin{tabular}{|c|c|c|c|c|c|c|}
\hline $\begin{array}{l}0.4 \\
1.3 \\
1.4 \\
0.4 \\
1.1\end{array}$ & $\begin{array}{r}3.9 \\
12.9 \\
12.4 \\
16.9 \\
7.7\end{array}$ & $\begin{array}{l}-- \\
-- \\
-- \\
--\end{array}$ & $\begin{array}{l}-- \\
-- \\
-- \\
-- \\
--\end{array}$ & $\begin{array}{l}-- \\
-- \\
-- \\
--\end{array}$ & $\begin{array}{l}-- \\
-- \\
-- \\
-- \\
--\end{array}$ & $\begin{array}{l}49.6 \\
45.0 \\
47.0 \\
41.3 \\
61.9\end{array}$ \\
\hline $\begin{array}{l}1.6 \\
1.6 \\
1.6 \\
1.0 \\
0.3\end{array}$ & $\begin{array}{r}6.5 \\
3.9 \\
4.6 \\
6.2 \\
13.4\end{array}$ & $\begin{array}{l}-- \\
-- \\
-- \\
--\end{array}$ & $\begin{array}{l}-- \\
-- \\
-- \\
-- \\
--\end{array}$ & $\begin{array}{l}-- \\
-- \\
-- \\
--\end{array}$ & $\begin{array}{l}-- \\
-- \\
-- \\
-- \\
--\end{array}$ & $\begin{array}{l}65.2 \\
74.1 \\
72.1 \\
73.2 \\
54.8\end{array}$ \\
\hline $\begin{array}{l}1.8 \\
0.6 \\
1.6 \\
1.4 \\
0.8\end{array}$ & $\begin{array}{r}2.8 \\
19.9 \\
2.8 \\
3.5 \\
14.0\end{array}$ & $\begin{array}{l}-- \\
-- \\
-- \\
--\end{array}$ & $\begin{array}{l}-- \\
-- \\
-- \\
-- \\
--\end{array}$ & $\begin{array}{l}-- \\
-- \\
-- \\
--\end{array}$ & $\begin{array}{l}-- \\
-- \\
-- \\
--\end{array}$ & $\begin{array}{l}85.7 \\
39.7 \\
82.2 \\
79.6 \\
51.0\end{array}$ \\
\hline $\begin{array}{l}0.5 \\
1.5 \\
1.7 \\
2.8 \\
1.4\end{array}$ & $\begin{array}{r}12.1 \\
6.8 \\
4.4 \\
4.3 \\
0.8\end{array}$ & $\begin{array}{l}-- \\
-- \\
-- \\
--\end{array}$ & $\begin{array}{l}-- \\
-- \\
-- \\
-- \\
--\end{array}$ & $\begin{array}{l}-- \\
-- \\
-- \\
--\end{array}$ & $\begin{array}{l}-- \\
-- \\
-- \\
-- \\
--\end{array}$ & $\begin{array}{l}54.6 \\
69.8 \\
76.0 \\
77.0 \\
87.7\end{array}$ \\
\hline $\begin{array}{l}1.9 \\
1.3 \\
0.5 \\
0.8 \\
2.6\end{array}$ & $\begin{array}{r}4.1 \\
16.8 \\
6.0 \\
10.7 \\
2.0\end{array}$ & $\begin{array}{l}-- \\
-- \\
-- \\
--\end{array}$ & $\begin{array}{l}-- \\
-- \\
-- \\
--\end{array}$ & $\begin{array}{l}-- \\
-- \\
-- \\
--\end{array}$ & $\begin{array}{l}-- \\
-- \\
-- \\
-- \\
--\end{array}$ & $\begin{array}{l}77.2 \\
42.9 \\
70.7 \\
59.4 \\
79.4\end{array}$ \\
\hline 2.1 & 1.7 & -- & -- & -- & -- & 81.4 \\
\hline $\begin{array}{l}0.6 \\
0.8 \\
1.9 \\
1.8\end{array}$ & $\begin{array}{r}17.2 \\
6.9 \\
9.2 \\
17.0\end{array}$ & $\begin{array}{l}-- \\
-- \\
--\end{array}$ & $\begin{array}{l}-- \\
-- \\
-- \\
--\end{array}$ & $\begin{array}{l}-- \\
-- \\
--\end{array}$ & $\begin{array}{l}-- \\
-- \\
-- \\
--\end{array}$ & $\begin{array}{l}43.3 \\
68.2 \\
56.2 \\
42.4\end{array}$ \\
\hline $\begin{array}{l}0.7 \\
2.3 \\
2.1 \\
1.1 \\
0.3\end{array}$ & $\begin{array}{r}4.0 \\
5.9 \\
0.2 \\
0.4 \\
22.5\end{array}$ & $\begin{array}{l}-- \\
-- \\
-- \\
--\end{array}$ & $\begin{array}{l}-- \\
-- \\
-- \\
-- \\
--\end{array}$ & $\begin{array}{l}-- \\
-- \\
-- \\
--\end{array}$ & $\begin{array}{l}-- \\
-- \\
-- \\
-- \\
--\end{array}$ & $\begin{array}{r}74.1 \\
72.8 \\
30.8 \\
81.2 \\
7.9\end{array}$ \\
\hline $\begin{array}{l}1.7 \\
0.4 \\
1.5 \\
0.7 \\
0.3\end{array}$ & $\begin{array}{r}2.2 \\
5.8 \\
1.1 \\
1.2 \\
29.2\end{array}$ & $\begin{array}{l}-- \\
-- \\
-- \\
--\end{array}$ & $\begin{array}{l}-- \\
-- \\
-- \\
--\end{array}$ & $\begin{array}{l}-- \\
-- \\
-- \\
--\end{array}$ & $\begin{array}{l}-- \\
-- \\
-- \\
-- \\
--\end{array}$ & $\begin{array}{l}80.0 \\
78.7 \\
46.6 \\
84.0 \\
20.2\end{array}$ \\
\hline $\begin{array}{l}0.9 \\
0.3 \\
0.5 \\
1.1\end{array}$ & $\begin{array}{r}2.1 \\
14.3 \\
2.2 \\
1.2\end{array}$ & $\begin{array}{l}-- \\
-- \\
--\end{array}$ & $\begin{array}{l}-- \\
-- \\
--\end{array}$ & $\begin{array}{l}-- \\
-- \\
--\end{array}$ & $\begin{array}{l}-- \\
-- \\
--\end{array}$ & $\begin{array}{l}79.3 \\
48.0 \\
80.6 \\
82.3\end{array}$ \\
\hline
\end{tabular}

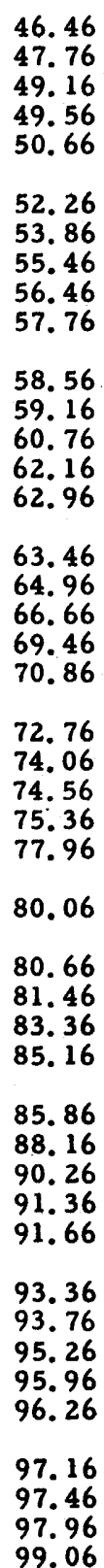




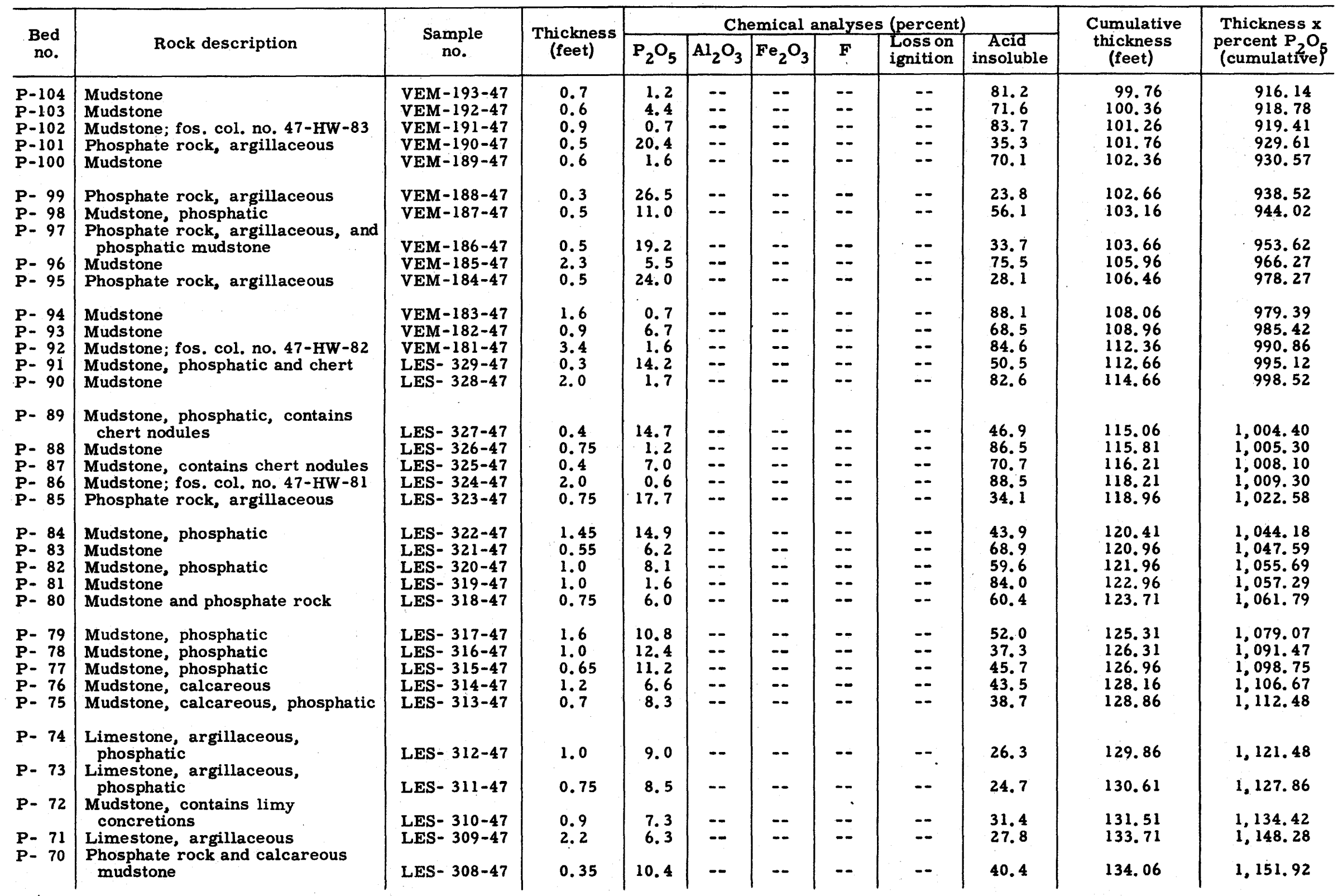




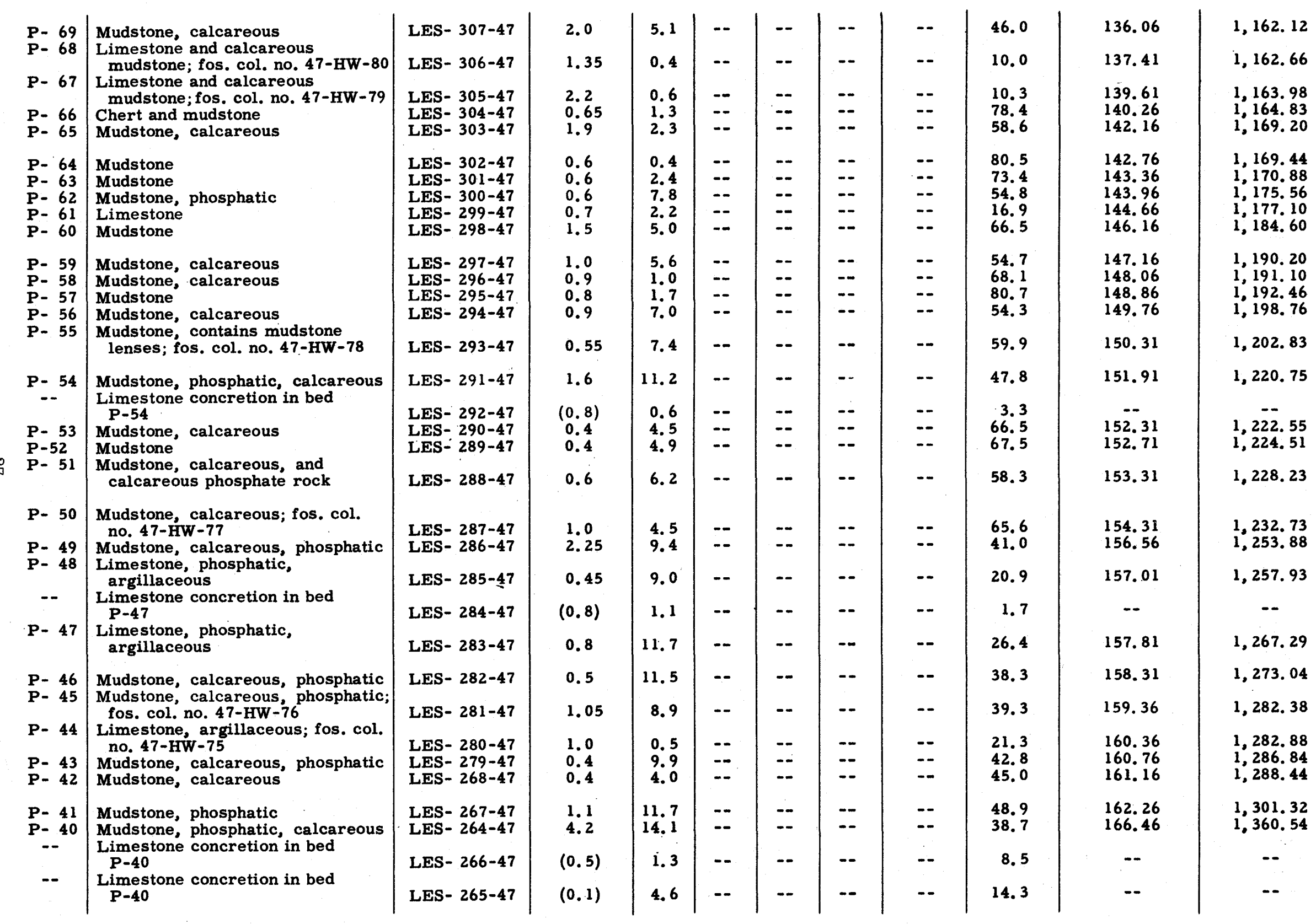




\begin{tabular}{|c|c|c|c|c|c|c|c|c|c|c|c|}
\hline \multirow{2}{*}{$\begin{array}{l}\text { Bed } \\
\text { no. }\end{array}$} & \multirow[b]{2}{*}{ Rock description } & \multirow{2}{*}{$\begin{array}{l}\text { Sample } \\
\text { no. }\end{array}$} & \multirow{2}{*}{$\begin{array}{c}\text { Thickness } \\
\text { (feet) }\end{array}$} & \multicolumn{6}{|c|}{ Chemical analyses (percent) } & \multirow{2}{*}{$\begin{array}{l}\text { Cumulative } \\
\text { thickness } \\
\text { (feet) }\end{array}$} & \multirow{2}{*}{$\begin{array}{l}\text { Thickness } x \\
\text { percent } \mathrm{P}_{2} \mathrm{O}_{5} \\
\text { (cumulative) }\end{array}$} \\
\hline & & & & $\mathrm{P}_{2} \mathrm{O}_{5}$ & $\mathrm{Al}_{2} \mathrm{O}_{3}$ & $\mathrm{Fe}_{2} \mathrm{O}_{3}$ & $\mathbf{F}$ & $\begin{array}{l}\text { Loss on } \\
\text { ignition }\end{array}$ & $\begin{array}{c}\text { Acid } \\
\text { insoluble }\end{array}$ & & \\
\hline $\begin{array}{l}P=39 \\
P-38 \\
P=37\end{array}$ & $\begin{array}{l}\text { Mudstone, calcareous, phosphatic } \\
\text { Mudstone, calcareous, phosphatic } \\
\text { Phosphate rock and calcareous }\end{array}$ & $\begin{array}{l}\text { LES- 263-47 } \\
\text { LES- } 262-47\end{array}$ & $\begin{array}{l}0.6 \\
0.65\end{array}$ & $\begin{array}{l}11.3 \\
12.1\end{array}$ & $\therefore$ & -- & -- & -- & $\begin{array}{l}41.3 \\
36.1\end{array}$ & $\begin{array}{l}167.06 \\
167.71\end{array}$ & $\begin{array}{l}1,367.32 \\
1,375.18\end{array}$ \\
\hline P- 36 & $\begin{array}{l}\text { mudstone } \\
\text { Phosphate rock, contains } \\
\text { laminations of calcareous }\end{array}$ & LES- $261-47$ & 2.5 & 15.8 & -- & - & -- & -- & 23. 7 & 170.21 & $1,414.68$ \\
\hline$P-35$ & $\begin{array}{l}\text { mudstone } \\
\text { Mudstone and phosphate rock }\end{array}$ & $\begin{array}{l}\text { LES- } 260-47 \\
\text { LES- } 259-47\end{array}$ & $\begin{array}{l}1.0 \\
0.55\end{array}$ & $\begin{array}{l}23.1 \\
24.5\end{array}$ & -- & -- & -- & -- & $\begin{array}{l}17.4 \\
22.3\end{array}$ & $\begin{array}{l}171.21 \\
171.76\end{array}$ & $\begin{array}{l}1,437.78 \\
1,451.26\end{array}$ \\
\hline $\begin{array}{l}P=34 \\
P=33\end{array}$ & $\begin{array}{l}\text { Phosphate rock, calcareous } \\
\text { Limestone, contains phosphate }\end{array}$ & LES- 278-47 & 0.5 & 28.8 & -- & -- & -- & -- & 4.6 & 172.26 & $1,465.66$ \\
\hline $\begin{array}{l}P-32 \\
P-31\end{array}$ & $\begin{array}{l}\text { rock lenses } \\
\text { Phosphate rock, calcareous } \\
\text { Phosphate rock and mudstone; }\end{array}$ & $\begin{array}{l}\text { LES- } 277-47 \\
\text { LES- } 276-47\end{array}$ & $\begin{array}{l}0.8 \\
0.55\end{array}$ & $\begin{array}{r}8.2 \\
24.2\end{array}$ & -- & -- & -- & -- & $\begin{array}{l}10.4 \\
13.4\end{array}$ & $\begin{array}{l}173.06 \\
173.61\end{array}$ & $\begin{array}{l}1,472.22 \\
1,485.52\end{array}$ \\
\hline & $\begin{array}{l}\text { fos. col. no. } 47-\mathrm{HW}-174 \\
\text { Phosphate rock and phosphatic } \\
\text { mudstone }\end{array}$ & $\begin{array}{l}\text { LES- } 275-47 \\
\text { LES- } 274-47\end{array}$ & $\begin{array}{l}1.15 \\
0.6\end{array}$ & $\begin{array}{l}28.4 \\
18.4\end{array}$ & -- & -- & -- & -- & $\begin{array}{l}12.9 \\
34.5\end{array}$ & $\begin{array}{l}174.76 \\
175.36\end{array}$ & $\begin{array}{l}1,518.18 \\
1,529.22\end{array}$ \\
\hline $\begin{array}{l}P=29 \\
P=28 \\
P=27 \\
P=26 \\
P=25\end{array}$ & $\begin{array}{l}\text { Limestone } \\
\text { Mudstone, phosphatic } \\
\text { Limestone } \\
\text { Limestone } \\
\text { Phosphate rock, argillaceous }\end{array}$ & $\begin{array}{l}\text { LES- } 273-47 \\
\text { LES- } 272-47 \\
\text { LES- } 271-47 \\
\text { LES- } 270-47 \\
\text { LES- } 269-47\end{array}$ & $\begin{array}{l}3.2 \\
1.75 \\
0.9 \\
2.85 \\
0.6\end{array}$ & $\begin{array}{r}4.6 \\
11.0 \\
3.0 \\
3.8 \\
21.8\end{array}$ & $\begin{array}{l}-- \\
-- \\
-- \\
4.5\end{array}$ & $\begin{array}{l}-- \\
-- \\
-- \\
1.6\end{array}$ & $\begin{array}{l}-- \\
\cdots \\
-- \\
1.99\end{array}$ & $\begin{array}{l}-- \\
-- \\
-- \\
-- \\
7.36\end{array}$ & $\begin{array}{l}13.7 \\
46.1 \\
15.0 \\
18.7 \\
29.9\end{array}$ & $\begin{array}{l}178.56 \\
180.31 \\
181.21 \\
184.06 \\
184.66\end{array}$ & $\begin{array}{l}1,543.94 \\
1,563.20 \\
1,565.90 \\
1,576.72 \\
1,589.80\end{array}$ \\
\hline & $\begin{array}{l}\text { Phosphate rock, calcareous, and } \\
\text { calcareous phosphatic mudstone }\end{array}$ & LES- 248-47 & 1.45 & 29.6 & 2.3 & 1.2 & 2.82 & 7.38 & 14.4 & 186.11 & $1,632.72$ \\
\hline $\begin{array}{l}P-23 \\
P=22 \\
P=21 \\
P=20\end{array}$ & $\begin{array}{l}\text { Phosphate rock and phosphatic } \\
\text { calcareous mudstone } \\
\text { Phosphate rock, argillaceous } \\
\text { Mudstone and phosphate rock } \\
\text { Mudstone }\end{array}$ & $\begin{array}{l}\text { LES- } 247-47 \\
\text { LES- } 246-47 \\
\text { LES- } 245-47 \\
\text { LES- } 244-47\end{array}$ & $\begin{array}{l}0.5 \\
0.55 \\
1.1 \\
0.4\end{array}$ & $\begin{array}{r}29.4 \\
25.1 \\
18.8 \\
6.7\end{array}$ & $\begin{array}{l}2.2 \\
3.3 \\
6.4 \\
9.40\end{array}$ & $\begin{array}{l}1.1 \\
1.5 \\
2.2 \\
2.97\end{array}$ & $\begin{array}{l}2.67 \\
2.25 \\
1.62 \\
0.57\end{array}$ & $\begin{array}{l}6.18 \\
6.74 \\
6.84 \\
5.92\end{array}$ & $\begin{array}{l}16.5 \\
26.1 \\
40.2 \\
70.2\end{array}$ & $\begin{array}{l}186.61 \\
187.16 \\
188.26 \\
188.66\end{array}$ & $\begin{array}{l}1,647.42 \\
1,661.23 \\
1,681.91 \\
1,684.59\end{array}$ \\
\hline $\begin{array}{l}P=19 \\
P-18 \\
P-17 \\
P=16 \\
P-15\end{array}$ & $\begin{array}{l}\text { Phosphate rock } \\
\text { Phosphate rock } \\
\text { Phosphate rock } \\
\text { Phosphate rock, argillaceous } \\
\text { Phosphate rock, argillaceous }\end{array}$ & $\begin{array}{l}\text { VEM-180-47 } \\
\text { VEM-179-47 } \\
\text { VEM-178-47 } \\
\text { VEM-177-47 } \\
\text { VEM-176-47 }\end{array}$ & $\begin{array}{l}1.0 \\
1.4 \\
0.9 \\
1.3 \\
1.2\end{array}$ & $\begin{array}{l}33.1 \\
27.8 \\
31.8 \\
26.2 \\
21.9\end{array}$ & $\begin{array}{l}1.3 \\
2.8 \\
1.7 \\
2.7 \\
5.4\end{array}$ & $\begin{array}{l}0.58 \\
1.1 \\
0.91 \\
1.2 \\
1.9\end{array}$ & $\begin{array}{l}2.49 \\
2.56 \\
2.84 \\
2.64 \\
1.92\end{array}$ & $\begin{array}{l}6.16 \\
6.70 \\
5.96 \\
6.90 \\
7.48\end{array}$ & $\begin{array}{l}8.6 \\
18.9 \\
10.4 \\
20.6 \\
31.4\end{array}$ & $\begin{array}{l}189.66 \\
191.06 \\
191.96 \\
193.26 \\
194.46\end{array}$ & $\begin{array}{l}1,717.69 \\
1,756.61 \\
1,785.23 \\
1,819.29 \\
1,845.57\end{array}$ \\
\hline$P-14$ & $\begin{array}{l}\text { Phosphate rock and phosphatic } \\
\text { mudstone }\end{array}$ & VEM-175-47 & 0.6 & 16.3 & 7.3 & 1.9 & 1.43 & 9.44 & 39.9 & 195.06 & $1,855.35$ \\
\hline $\begin{array}{l}P=13 \\
P=12 \\
P=11 \\
P=10\end{array}$ & $\begin{array}{l}\text { Phosphate rock, argillaceous; } \\
\text { fos, col. no. 47-HW-74 } \\
\text { Phosphate rock, argillaceous } \\
\text { Phosphate rock } \\
\text { Phosphate rock }\end{array}$ & $\begin{array}{l}\text { RAH- 189-47 } \\
\text { WOM - 3182 } \\
\text { WOM - 3181 } \\
\text { RAH- 186-47 }\end{array}$ & $\begin{array}{l}2.5 \\
1.35 \\
2.1 \\
1.9\end{array}$ & $\begin{array}{l}24.0 \\
25.4 \\
33.0 \\
33.3\end{array}$ & $\begin{array}{l}3.9 \\
1.3 \\
3.5 \\
0.7\end{array}$ & $\begin{array}{l}1.5 \\
0.55 \\
1.7 \\
0.52\end{array}$ & $\begin{array}{l}2.08 \\
3.25 \\
2.55 \\
3.40\end{array}$ & $\begin{array}{l}5.76 \\
6.24 \\
5.24 \\
6.24\end{array}$ & $\begin{array}{r}26.3 \\
25.5 \\
6.9 \\
4.3\end{array}$ & $\begin{array}{l}197.56 \\
198.91 \\
201.01 \\
202.91\end{array}$ & $\begin{array}{l}1,915.35 \\
1,949.64 \\
1,018.94 \\
2,082.21\end{array}$ \\
\hline $\begin{array}{ll}P- & 9 \\
P- & 8\end{array}$ & $\begin{array}{l}\text { Phosphate rock } \\
\text { Phosphate rock }\end{array}$ & $\begin{array}{l}\text { RAH- } 185-47 \\
\text { RAH- } 184-47\end{array}$ & $\begin{array}{l}1.7 \\
0.4\end{array}$ & $\begin{array}{l}35.0 \\
26.8\end{array}$ & 2.3 & $1 . \overline{0}$ & $\begin{array}{l}3.60 \\
2.72\end{array}$ & 6.18 & $\begin{array}{r}3.0 \\
18.0\end{array}$ & $\begin{array}{l}204.61 \\
205.01\end{array}$ & $\begin{array}{l}2,141.71 \\
2,152.43\end{array}$ \\
\hline
\end{tabular}




\begin{tabular}{|c|c|c|c|c|c|c|c|c|c|c|c|}
\hline $\begin{array}{ll}\text { P- } & 7 \\
\text { P- } & 6 \\
\text { P- } & 5 \\
\text { P- } & 4 \\
\text { P- } & 3\end{array}$ & $\begin{array}{l}\text { Phosphate rock } \\
\text { Mudstone } \\
\text { Mudstone } \\
\text { Limestone, argillaceous } \\
\text { Mudstone, calcareous }\end{array}$ & $\begin{array}{l}\text { RAH- } 183-47 \\
\text { RAH- } 182-47 \\
\text { RAH- } 181-47 \\
\text { RAH- } 180-47 \\
\text { RAH- } 179-47\end{array}$ & $\begin{array}{l}0.55 \\
2.1 \\
1.0 \\
0.4 \\
0.5\end{array}$ & $\begin{array}{r}28.3 \\
2.5 \\
0.8 \\
0.6 \\
3.9\end{array}$ & $\begin{array}{r}2.2 \\
11.6 \\
11.6 \\
4.5 \\
8.8\end{array}$ & $\begin{array}{l}0.95 \\
3.0 \\
3.4 \\
1.7 \\
6.3\end{array}$ & $\begin{array}{l}2.39 \\
-- \\
=- \\
--\end{array}$ & $\begin{array}{r}6.46 \\
10.24 \\
6.56 \\
31.28 \\
9.36\end{array}$ & $\begin{array}{l}16.6 \\
68.4 \\
77.8 \\
30.3 \\
56.3\end{array}$ & $\begin{array}{l}205.56 \\
207.66 \\
208.66 \\
209.06 \\
209.56\end{array}$ & $\begin{array}{l}2,168.00 \\
2,173.24 \\
2,174.04 \\
2,174.28 \\
2,176.24\end{array}$ \\
\hline $\begin{array}{ll}\text { P- } & 2 \\
\text { P- } & 1\end{array}$ & $\begin{array}{l}\text { Phosphate rock } \\
\text { Phosphate rock }\end{array}$ & $\begin{array}{l}\text { RMC }-133-47 \\
\text { RMC }-134-47\end{array}$ & $\begin{array}{l}1.1 \\
0.5\end{array}$ & $\begin{array}{l}30.2 \\
31.4\end{array}$ & $\begin{array}{l}2.2 \\
1.3\end{array}$ & $\begin{array}{l}1.4 \\
0.75\end{array}$ & $\begin{array}{l}-- \\
--\end{array}$ & $\begin{array}{l}5.66 \\
4.48\end{array}$ & $\begin{array}{r}12.8 \\
9.9\end{array}$ & $\begin{array}{l}210.66 \\
211.16\end{array}$ & $\begin{array}{l}2,209.46 \\
2,225.16\end{array}$ \\
\hline \multicolumn{12}{|c|}{ Wells formation } \\
\hline$C w-1$ & Limestone & RAH- $177-47$ & 2.0 & 1.2 & -- & -- & -- & -- & 4.7 & -- & -- \\
\hline
\end{tabular}


Semi-quantitative analyses of selected samples of the phosphatic shale member of the Phosphoria formation, Trail Canyon, Idaho (see immediately preceding pages for location of section, thickness and description of strata, and chemical analyses of samples), made by

U. S. Geological Survey Laboratory, Geochemistry and Petrology Branch, Washington, D. C. In addition to the elements listed in the table below, Sb, As, Bi, Cs, Cb, Dy, Er, Eu, Ga, Gd, Au, Ho, In, Li, Lu, Nd, Pt, Pr, Rb, Sm, Sc, Ta, Te, Tb, Tl, Tm, W, and Yb were looked for in all samples but were not detected.

\section{Explanation of symbols}

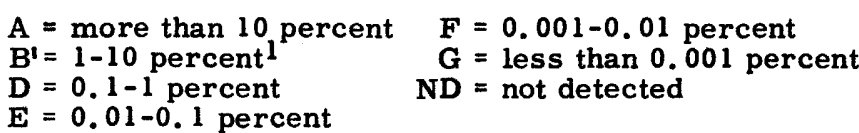

\begin{tabular}{r|l|l|l|l|l|l|l|l|l|l|l|l|l|l|l|l|l|l|l|l|l|l|l|l|l|l|l|l|l|l|}
\hline $\begin{array}{c}\text { Bed } \\
\text { no. }\end{array}$ & Sample no. & Al & Ba & Be & B & Cd & Ca & Cr & Co & Cu & Fe & La & Pb & Mg & Mn & Mo & Ni & P & Si & Ag & Na & Sr & Sn & Ti & V & Y & Zn & Zr \\
\hline
\end{tabular}




\begin{tabular}{|c|c|c|c|c|c|c|c|c|c|c|c|c|c|c|c|c|c|c|c|c|c|c|c|c|c|c|c|}
\hline $\begin{array}{l}P-19 \\
P-18 \\
P-17 \\
P=16 \\
P=15\end{array}$ & $\begin{array}{l}\text { VEM-180-47 } \\
\text { VEM-1 } 79-47 \\
\text { VEM-1 78-47 } \\
\text { VEM-1 } 77-47 \\
\text { VEM-1 } \\
\text { VE-47 }\end{array}$ & $\begin{array}{l}\mathbf{B}^{\prime} \\
\mathbf{B}^{\prime} \\
\mathbf{B}^{\prime} \\
\mathbf{B}^{\prime} \\
\mathbf{B}^{\prime}\end{array}$ & $\begin{array}{l}\mathbf{E} \\
\mathbf{E} \\
\mathbf{E} \\
\mathbf{E} \\
\mathbf{E}\end{array}$ & $\begin{array}{c}\text { ND } \\
\mathbf{G} \\
\mathbf{G} \\
\mathrm{ND} \\
\mathbf{N D}\end{array}$ & $\begin{array}{l}\text { ND } \\
\mathbf{F} \\
\mathbf{F} \\
\mathbf{F} \\
\mathbf{F}\end{array}$ & $\begin{array}{l}\mathbf{E} \\
\mathbf{E} \\
\mathbf{E} \\
\mathbf{E} \\
\mathbf{E}\end{array}$ & $\begin{array}{l}\text { A } \\
\mathbf{A} \\
\mathbf{A} \\
\mathbf{A} \\
\mathbf{A}\end{array}$ & $\begin{array}{l}\mathbf{D} \\
\mathbf{D} \\
\mathbf{D} \\
\mathbf{D} \\
\mathbf{D}\end{array}$ & $\begin{array}{l}\text { ND } \\
\text { ND } \\
\text { ND } \\
\text { ND } \\
\text { ND }\end{array}$ & $\begin{array}{l}\mathbf{E} \\
\mathbf{E} \\
\mathbf{E} \\
\mathbf{E} \\
\mathbf{E}\end{array}$ & $\begin{array}{l}\text { D } \\
\mathbf{D} \\
\mathbf{D} \\
\mathbf{D} \\
\mathbf{D}\end{array}$ & $\begin{array}{l}\mathbf{F} \\
\mathbf{F} \\
\mathbf{F} \\
\mathbf{E} \\
\mathbf{E}\end{array}$ & $\begin{array}{l}\mathbf{F} \\
\mathbf{E} \\
\mathbf{E} \\
\mathbf{E} \\
\mathbf{E}\end{array}$ & $\begin{array}{l}\text { D } \\
\text { D } \\
\text { D } \\
D \\
D\end{array}$ & $\begin{array}{l}\mathbf{F} \\
\mathbf{F} \\
\mathbf{F} \\
\mathbf{F} \\
\mathbf{E}\end{array}$ & $\begin{array}{l}\mathbf{F} \\
\mathbf{F} \\
\mathbf{F} \\
\mathbf{F} \\
\mathbf{F}\end{array}$ & $\begin{array}{l}\mathbf{F} \\
\mathbf{E} \\
\mathbf{E} \\
\mathbf{E} \\
\mathbf{E}\end{array}$ & $\begin{array}{l}\text { A } \\
\mathbf{A} \\
\mathbf{A} \\
\mathbf{A} \\
\mathbf{A}\end{array}$ & $\begin{array}{l}\mathbf{B}^{\prime} \\
\mathbf{B}^{\prime} \\
\mathbf{B}^{\prime} \\
\mathbf{B}^{\prime} \\
\mathbf{B}^{\prime}\end{array}$ & $\begin{array}{l}\mathbf{G} \\
\mathbf{G} \\
\mathbf{G} \\
\mathbf{F} \\
\mathbf{F}\end{array}$ & $\begin{array}{l}\mathbf{D} \\
\mathbf{D} \\
\mathbf{D} \\
\mathbf{D} \\
\mathbf{D}\end{array}$ & D & $\begin{array}{l}\mathbf{F} \\
\mathbf{F} \\
\mathbf{F} \\
\mathbf{F} \\
\mathbf{F}\end{array}$ & $\begin{array}{l}\mathbf{E} \\
\mathbf{E} \\
\mathbf{E} \\
\mathbf{E} \\
\mathbf{D}\end{array}$ & $\mathbf{E}$ & $\mathbf{F}$ & \\
\hline $\begin{array}{l}P-14 \\
P-13 \\
P-12 \\
P-11 \\
P-10\end{array}$ & $\begin{array}{l}\text { VEM-1 } 75-47 \\
\text { RAH- } 189-47 \\
\text { WOM- } 3182 \\
\text { WOM- } 3181 \\
\text { RAH- } 186-47\end{array}$ & $\begin{array}{l}\mathbf{B}^{\prime} \\
\mathbf{B}^{\prime} \\
\mathbf{B}^{\prime} \\
\mathbf{B}^{\prime} \\
\mathbf{B}^{\prime}\end{array}$ & $\begin{array}{l}\mathbf{E} \\
\mathbf{E} \\
\mathbf{E} \\
\mathbf{E} \\
\mathbf{E}\end{array}$ & $\begin{array}{l}\text { ND } \\
\text { ND } \\
\text { ND } \\
\text { ND } \\
\text { ND }\end{array}$ & $\begin{array}{l}\mathbf{E} \\
\mathbf{F} \\
\mathbf{F} \\
\mathbf{F} \\
\mathbf{F}\end{array}$ & $\begin{array}{l}\mathbf{E} \\
\mathbf{E} \\
\mathbf{E} \\
\mathbf{F} \\
\mathbf{E}\end{array}$ & $\begin{array}{l}\mathbf{A} \\
\mathbf{A} \\
\mathbf{A} \\
\mathbf{A} \\
\mathbf{A}\end{array}$ & $\begin{array}{l}\mathbf{D} \\
\mathbf{D} \\
\mathbf{D} \\
\mathbf{E} \\
\mathbf{D}\end{array}$ & $\begin{array}{l}\text { ND } \\
\text { ND } \\
\text { ND } \\
\text { ND }\end{array}$ & $\begin{array}{l}\mathbf{E} \\
\mathbf{E} \\
\mathbf{E} \\
\mathbf{E} \\
\mathbf{E}\end{array}$ & $\begin{array}{l}\text { D } \\
\text { D } \\
\text { D } \\
\mathbf{D} \\
\text { D }\end{array}$ & $\begin{array}{l}\mathbf{E} \\
\mathbf{E} \\
\mathbf{E} \\
\mathbf{E} \\
\mathbf{E}\end{array}$ & $\begin{array}{l}\mathbf{E} \\
\mathbf{E} \\
\mathbf{E} \\
\mathbf{F} \\
\mathbf{E}\end{array}$ & $\begin{array}{l}\text { D } \\
\text { D } \\
\text { D } \\
\text { D } \\
\text { D }\end{array}$ & $\begin{array}{l}\mathbf{E} \\
\mathbf{E} \\
\mathbf{F} \\
\mathbf{E} \\
\mathbf{F}\end{array}$ & $\begin{array}{l}\mathbf{F} \\
\mathbf{F} \\
\mathbf{F} \\
\mathbf{F} \\
\mathbf{F}\end{array}$ & $\begin{array}{l}\mathbf{E} \\
\mathbf{E} \\
\mathbf{E} \\
\mathbf{E} \\
\mathbf{E}\end{array}$ & $\begin{array}{l}\text { A } \\
\text { A } \\
\text { A } \\
\text { A } \\
\text { A }\end{array}$ & $\begin{array}{l}\mathbf{B}^{\prime} \\
\mathbf{B}^{\prime} \\
\mathbf{B}^{\prime} \\
\mathbf{B}^{\prime} \\
\mathbf{B}^{\prime}\end{array}$ & $\begin{array}{l}\mathbf{F} \\
\mathrm{F}\end{array}$ & $\begin{array}{l}\mathbf{D} \\
\mathbf{D} \\
\mathbf{D} \\
\mathbf{D} \\
\mathbf{D}\end{array}$ & & $\begin{array}{l}\mathbf{F} \\
\mathbf{F} \\
\mathbf{F} \\
\mathbf{F} \\
\mathbf{F}\end{array}$ & $\begin{array}{l}\mathbf{D} \\
\mathbf{D} \\
\mathbf{E} \\
\mathbf{D} \\
\mathbf{E}\end{array}$ & 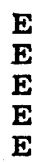 & $\mathbf{L}$ & \\
\hline $\begin{array}{ll}P- & 9 \\
P- & 8 \\
P- & 7 \\
P- & 6 \\
P- & 5\end{array}$ & $\begin{array}{l}\text { RAH- } 185-47 \\
\text { RAH- } 184-47 \\
\text { RAH- } 183-47 \\
\text { RAH- } 182-47 \\
\text { RAH- } 181-47\end{array}$ & $\begin{array}{l}\mathbf{D} \\
\mathbf{B}^{\prime} \\
\mathbf{B}^{\prime} \\
\mathbf{B}^{\prime} \\
\mathbf{B}^{\prime}\end{array}$ & $\begin{array}{l}\mathbf{E} \\
\mathbf{E} \\
\mathbf{E} \\
\mathbf{D} \\
\mathbf{E}\end{array}$ & $\begin{array}{l}\text { ND } \\
\text { ND } \\
\text { ND } \\
\text { G } \\
\text { G }\end{array}$ & $\begin{array}{l}\mathbf{F} \\
\mathbf{F} \\
\mathbf{F} \\
\mathbf{E} \\
\mathbf{E}\end{array}$ & $\begin{array}{l}\mathbf{E} \\
\mathbf{E} \\
\mathbf{E} \\
\mathbf{E} \\
\mathbf{E}\end{array}$ & $\begin{array}{c}\mathbf{A} \\
\mathbf{A} \\
\mathbf{A} \\
\mathbf{B}^{\prime} \\
\mathbf{B}^{\prime}\end{array}$ & $\begin{array}{l}\mathbf{D} \\
\mathbf{D} \\
\mathbf{D} \\
\mathbf{D} \\
\mathbf{D}\end{array}$ & $\begin{array}{l}\text { ND } \\
\text { ND } \\
\text { ND } \\
\text { ND } \\
\text { ND }\end{array}$ & $\begin{array}{l}\mathbf{E} \\
\mathbf{E} \\
\mathbf{E} \\
\mathbf{E} \\
\mathbf{E}\end{array}$ & $\begin{array}{l}\mathrm{D} \\
\mathrm{D} \\
\mathrm{D} \\
\mathrm{D} \\
\mathrm{D}\end{array}$ & $\begin{array}{l}\mathbf{F} \\
\mathbf{F} \\
\mathbf{F} \\
\text { ND } \\
\text { ND }\end{array}$ & $\begin{array}{l}\mathbf{E} \\
\mathbf{E} \\
\mathbf{E} \\
\mathbf{F} \\
\mathbf{F}\end{array}$ & $\begin{array}{l}D \\
D \\
D \\
D \\
D\end{array}$ & $\begin{array}{l}\mathbf{F} \\
\mathbf{F} \\
\mathbf{F} \\
\mathbf{E} \\
\mathbf{E}\end{array}$ & $\begin{array}{l}\mathbf{F} \\
\mathbf{F} \\
\mathbf{F} \\
\mathbf{F} \\
\mathbf{F}\end{array}$ & $\begin{array}{l}\mathbf{E} \\
\mathbf{E} \\
\mathbf{E} \\
\mathbf{E} \\
\mathbf{E}\end{array}$ & $\begin{array}{l}\text { A } \\
\text { A } \\
\text { A } \\
\text { B }^{\prime} \\
\text { D }\end{array}$ & $\begin{array}{l}\mathbf{B}^{\prime} \\
\mathbf{B}^{\prime} \\
\mathbf{B}^{\prime} \\
\mathbf{A} \\
\mathbf{A}\end{array}$ & & $\begin{array}{l}\mathbf{D} \\
\mathbf{D} \\
\mathbf{D} \\
\mathbf{D} \\
\mathbf{D}\end{array}$ & & $\begin{array}{l}\mathbf{F} \\
\mathbf{F} \\
\text { ND } \\
\mathbf{F} \\
\mathbf{F}\end{array}$ & $\begin{array}{l}\mathbf{E} \\
\mathbf{E} \\
\mathbf{E} \\
\mathbf{D} \\
\mathbf{D}\end{array}$ & 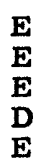 & $F$ & \\
\hline $\begin{array}{ll}P- & 4 \\
P- & 3 \\
P- & 2 \\
P- & 1\end{array}$ & $\begin{array}{l}\text { RAH- } 180-47 \\
\text { RAH- } 179-47 \\
\text { RMC-1 33-47 } \\
\text { RMC-134-47 }\end{array}$ & $\begin{array}{l}\mathbf{B}^{\prime} \\
\mathbf{B}^{\prime} \\
\mathbf{B}^{\prime} \\
\mathbf{B}^{\prime}\end{array}$ & $\begin{array}{l}\mathbf{E} \\
\mathbf{D} \\
\mathbf{E} \\
\mathbf{E}\end{array}$ & $\begin{array}{c}\text { G } \\
\text { G } \\
\text { ND } \\
\text { ND }\end{array}$ & $\begin{array}{l}\mathbf{F} \\
\mathbf{F} \\
\mathbf{F} \\
\mathbf{F}\end{array}$ & $\begin{array}{l}\mathbf{E} \\
\mathbf{E} \\
\mathbf{E} \\
\mathbf{E}\end{array}$ & $\begin{array}{l}\mathbf{B}^{\mathbf{t}} \\
\mathbf{B}^{\mathbf{t}} \\
\mathbf{A} \\
\mathbf{A}\end{array}$ & $\begin{array}{l}\mathbf{E} \\
\mathbf{D} \\
\mathbf{D} \\
\mathbf{D}\end{array}$ & $\begin{array}{c}\text { ND } \\
\mathbf{E} \\
\mathbf{N D} \\
\mathbf{N D}\end{array}$ & $\begin{array}{l}\mathbf{F} \\
\mathbf{E} \\
\mathbf{F} \\
\mathbf{F}\end{array}$ & $\begin{array}{l}\mathbf{D} \\
\mathbf{D} \\
\mathrm{D} \\
\mathrm{D}\end{array}$ & $\begin{array}{c}\mathbf{N D} \\
\mathbf{F} \\
\mathbf{E} \\
\mathbf{E}\end{array}$ & $\begin{array}{l}\mathbf{F} \\
\mathbf{F} \\
\mathbf{E} \\
\mathbf{E}\end{array}$ & $\begin{array}{l}\mathbf{B}^{\mathbf{t}} \\
\mathbf{D} \\
\mathbf{D} \\
\mathbf{D}\end{array}$ & $\begin{array}{c}\mathbf{D} \\
\mathbf{B}^{\prime} \\
\mathbf{E} \\
\mathbf{F}\end{array}$ & $\begin{array}{l}\mathbf{F} \\
\mathbf{E} \\
\mathbf{F} \\
\mathbf{F}\end{array}$ & $\begin{array}{c}\mathbf{E} \\
\mathbf{B}^{\prime} \\
\mathbf{E} \\
\mathbf{E}\end{array}$ & $\begin{array}{l}\mathbf{D} \\
\mathbf{B} \\
\mathbf{A} \\
\mathbf{A}\end{array}$ & $\begin{array}{c}\mathbf{A} \\
\mathbf{A} \\
\mathbf{B}^{\prime} \\
\mathbf{B}^{\prime}\end{array}$ & & $\begin{array}{l}\mathbf{D} \\
\mathbf{D} \\
\mathbf{D} \\
\mathbf{D}\end{array}$ & & $\begin{array}{l}\mathbf{F} \\
\mathbf{E} \\
\mathbf{F} \\
\mathbf{F}\end{array}$ & $\begin{array}{l}\mathbf{E} \\
\mathbf{D} \\
\mathbf{E} \\
\mathbf{E}\end{array}$ & & $\mathbf{N}$ & \\
\hline
\end{tabular}


JOHNSON CREEK, IDAHO. LOT NO. 1209.

Phosphatic shale member of Phosphoria formation sampled in two bulldozer trenches on north side of Johnson Creek, SE $\frac{1}{4}$ sec. 23, T. 8 S., R. 43 E., Caribou County, Idaho, near north end of east limb of Aspen Range anticline. Beds strike N. 10-40 $\mathrm{W}$. and dip $25-45^{\circ} \mathrm{NE}$. Section measured by

R. M. Campbell, R. A. Gulbrandsen, R. A. Hoppin, V. E. McKelvey, and R. A. Weeks and sampled by Campbell and Weeks in September and October 1947. Samples analyzed for $\mathrm{P}_{2} \mathrm{O}_{5}$ and acid insoluble by U. S. Bureau of Mines Laboratory, Albany, Oregon, and for other constituents by Trace Elements

Laboratory, U. S. Geological Survey, Washington, D. C.

\begin{tabular}{|c|c|c|c|c|c|c|c|c|c|c|}
\hline \multirow{2}{*}{$\begin{array}{c}\text { Bed } \\
\text { no. }\end{array}$} & \multirow{2}{*}{ Rock description } & \multirow{2}{*}{$\begin{array}{l}\text { Sample } \\
\text { no. }\end{array}$} & \multirow{2}{*}{$\begin{array}{l}\text { Thickness } \\
\text { (feet) }\end{array}$} & \multicolumn{5}{|c|}{ Chemical analyses (percent) } & \multirow{2}{*}{$\begin{array}{l}\text { Cumulative } \\
\text { thickness } \\
\text { (feet) }\end{array}$} & \multirow{2}{*}{$\begin{array}{l}\text { Thickness } x \\
\text { percent } \mathrm{P}_{2} \mathrm{O}_{5} \\
\text { (cumulative) }\end{array}$} \\
\hline & & & & $\mathbf{P}_{2} \mathrm{O}_{5}$ & $\mathrm{Al}_{2} \mathrm{O}_{3}$ & $\mathrm{Fe}_{2} \mathrm{O}_{3}$ & $\begin{array}{l}\text { Loss on } \\
\text { ignition }\end{array}$ & $\begin{array}{c}\text { Acid } \\
\text { insoluble }\end{array}$ & & \\
\hline \multicolumn{11}{|c|}{ Rex member of Phosphoria formation-basal beds only } \\
\hline $\begin{array}{ll}\mathbf{R}- & 2 \\
\mathbf{R}- & 1\end{array}$ & $\begin{array}{l}\text { Chert } \\
\text { Chert and mudstone }\end{array}$ & $\begin{array}{l}\text { RAW- 73-47 } \\
\text { RAW- 72-47 }\end{array}$ & $\begin{array}{l}8.1 \\
3.5\end{array}$ & $\begin{array}{l}0.7 \\
1.2\end{array}$ & -- & -- & -- & $\begin{array}{l}93.0 \\
81.2\end{array}$ & $\begin{array}{r}8.1 \\
11.6\end{array}$ & $\begin{array}{l}5.67 \\
9.87\end{array}$ \\
\hline \multicolumn{11}{|c|}{ Phosphatic shale member of Phosphoria formation } \\
\hline $\begin{array}{l}P-119 \\
P-118 \\
P-117 \\
P-116 \\
P-115\end{array}$ & $\begin{array}{l}\text { Mudstone } \\
\text { Phosphate rock, calcareous; fos. } \\
\text { col. no. 47-HW-2301 } \\
\text { Mudstone } \\
\text { Mudstone } \\
\text { Limestone, argillaceous }\end{array}$ & $\begin{array}{l}\text { RAW- } 71-47 \\
\text { RAW- } 70-47 \\
\text { RAW- } 69-47 \\
\text { RAW- } 68-47 \\
\text { RAW- } 67-47\end{array}$ & $\begin{array}{l}0.6 \\
0.5 \\
1.5 \\
1.4 \\
1.0\end{array}$ & $\begin{array}{r}2.9 \\
27.6 \\
2.4 \\
1.5 \\
5.7\end{array}$ & $\begin{array}{l}-- \\
-- \\
-- \\
--\end{array}$ & $\begin{array}{l}-- \\
-- \\
-- \\
--\end{array}$ & $\begin{array}{l}-- \\
-- \\
-- \\
--\end{array}$ & $\begin{array}{r}91.7 \\
7.6 \\
84.0 \\
75.6 \\
23.2\end{array}$ & $\begin{array}{l}0.6 \\
1.1 \\
2.6 \\
4.0 \\
5.0\end{array}$ & $\begin{array}{r}1.74 \\
15.54 \\
19.14 \\
21.24 \\
26.94\end{array}$ \\
\hline $\begin{array}{l}P-114 \\
P-113\end{array}$ & $\begin{array}{l}\text { Mudstone; fos. col. no. } 47-\mathrm{HW}-229 \\
\text { Mudstone, calcareous; fos. col. no. }\end{array}$ & RA.W- 66-47 & 0.8 & 2.1 & -- & -- & -- & 75.0 & 5.8 & 28.62 \\
\hline $\begin{array}{l}P-1 \\
P-1\end{array}$ & $\begin{array}{l}\text { 47-HW-228 } \\
\text { Mudstone; fos. col. no. } 47-\mathrm{HW}-227 \\
\text { Phosphate rock } \\
\text { Mudstone; fos. col. no. } 47-\mathrm{HW}-226\end{array}$ & $\begin{array}{l}\text { RAW- } 65-47 \\
\text { RAW- 64-47 } \\
\text { RAW- 63-47 } \\
\text { RAW- 62-47 }\end{array}$ & $\begin{array}{l}2.6 \\
2.4 \\
0.8 \\
2.1\end{array}$ & $\begin{array}{r}0.9 \\
3.2 \\
30.7 \\
6.4\end{array}$ & $\begin{array}{l}-- \\
-- \\
--\end{array}$ & $\begin{array}{l}-- \\
-- \\
--\end{array}$ & $\begin{array}{l}-- \\
-- \\
--\end{array}$ & $\begin{array}{l}65.2 \\
71.7 \\
13.3 \\
64.6\end{array}$ & $\begin{array}{l}8.4 \\
10.8 \\
11.6 \\
13.7\end{array}$ & $\begin{array}{l}30.96 \\
38.64 \\
63.20 \\
76.64\end{array}$ \\
\hline $\begin{array}{l}P-109 \\
P-108 \\
P-107 \\
P-106 \\
P-105\end{array}$ & $\begin{array}{l}\text { Mudstone; fos. col. no. } 47-\mathrm{HW}-225 \\
\text { Phosphate rock } \\
\text { Phosphate rock } \\
\text { Phosphate rock } \\
\text { Phosphate rock }\end{array}$ & $\begin{array}{l}\text { RAW- } 57-47 \\
\text { RAW- 56-47 } \\
\text { RAW- 55-47 } \\
\text { RAW- 54-47 } \\
\text { RAW- } 53-47\end{array}$ & $\begin{array}{l}0.5 \\
0.6 \\
0.4 \\
0.4 \\
0.8\end{array}$ & $\begin{array}{l}1.9 \\
36.9 \\
37.4 \\
35.3 \\
36.5\end{array}$ & $\begin{array}{l}-- \\
0.63 \\
0.53 \\
2.0 \\
0.82\end{array}$ & $\begin{array}{l}-- \\
0.46 \\
0.41 \\
0.87 \\
0.50\end{array}$ & $\begin{array}{l}-. \\
1.84 \\
1.32 \\
3.88 \\
1.96\end{array}$ & $\begin{array}{r}79.4 \\
5.6 \\
3.6 \\
4.0 \\
4.5\end{array}$ & $\begin{array}{l}14.2 \\
14.8 \\
15.2 \\
15.6 \\
16.4\end{array}$ & $\begin{array}{r}77.59 \\
99.73 \\
114.69 \\
128.81 \\
158.01\end{array}$ \\
\hline $\begin{array}{l}P-104 \\
P-103 \\
P-102 \\
P-101 \\
P-100\end{array}$ & $\begin{array}{l}\text { Phosphate rock } \\
\text { Phosphate rock and mudstone } \\
\text { Phosphate rock } \\
\text { Phosphate rock } \\
\text { Phosphate rock }\end{array}$ & $\begin{array}{l}\text { RAW- } 52-47 \\
\text { VEM-493-47 } \\
\text { VEM-492-47 } \\
\text { VEM-491-47 } \\
\text { VEM-490-47 }\end{array}$ & $\begin{array}{l}0.9 \\
0.5 \\
0.6 \\
0.5 \\
0.5\end{array}$ & $\begin{array}{l}35.3 \\
26.5 \\
34.6 \\
30.3 \\
33.0\end{array}$ & $\begin{array}{l}1.4 \\
3.2 \\
1.0 \\
2.1 \\
1.7\end{array}$ & $\begin{array}{l}0.70 \\
1.28 \\
0.60 \\
0.90 \\
1.22\end{array}$ & $\begin{array}{l}1.68 \\
2.98 \\
0.80 \\
4.46 \\
3.24\end{array}$ & $\begin{array}{r}7.3 \\
23.6 \\
9.9 \\
14.8 \\
11.5\end{array}$ & $\begin{array}{l}17.3 \\
17.8 \\
18.4 \\
18.9 \\
19.4\end{array}$ & $\begin{array}{l}189.78 \\
203.03 \\
223.79 \\
238.94 \\
255.44\end{array}$ \\
\hline $\begin{array}{l}P-99 \\
P-98 \\
P-97 \\
P-96 \\
P-95\end{array}$ & $\begin{array}{l}\text { Phosphate rock, argillaceous } \\
\text { Mudstone } \\
\text { Mudstone, phosphatic } \\
\text { Phosphate rock } \\
\text { Phosphate rock }\end{array}$ & $\begin{array}{l}\text { VEM-489-47 } \\
\text { VEM-488-47 } \\
\text { VEM-487-47 } \\
\text { VEM-486-47 } \\
\text { VEM-485-47 }\end{array}$ & $\begin{array}{l}0.6 \\
1.0 \\
1.3 \\
0.6 \\
0.6\end{array}$ & $\begin{array}{r}19.7 \\
5.5 \\
9.5 \\
28.9 \\
31.8\end{array}$ & $\begin{array}{c}7.5 \\
11 . \\
9.4 \\
4.1 \\
1.3\end{array}$ & $\begin{array}{l}2.26 \\
3.20 \\
1.88 \\
1.59 \\
0.30\end{array}$ & $\begin{array}{r}7.22 \\
11.12 \\
10.78 \\
7.00 \\
5.40\end{array}$ & $\begin{array}{l}35.1 \\
61.4 \\
55.3 \\
15.5 \\
11.7\end{array}$ & $\begin{array}{l}20.0 \\
21.0 \\
22.3 \\
22.9 \\
23.5\end{array}$ & $\begin{array}{l}267.26 \\
272.76 \\
285.11 \\
302.45 \\
321.53\end{array}$ \\
\hline P- 94 & Phosphate rock & VEM-484-47| & 0.6 & 34.5 & 1.1 & 0.43 & 4.86 & 6.0 & 24.1 & 342.23 \\
\hline
\end{tabular}




\begin{tabular}{|c|c|}
\hline P- 93 & Phosphate rock \\
\hline P- 92 & Phosphate rock \\
\hline $\begin{array}{l}P-91 \\
P-90\end{array}$ & $\begin{array}{l}\text { Mudstone; fos, col. no. } 47-\mathrm{HW}-303 \\
\text { Phosphate rock, argillaceous; fos. col. }\end{array}$ \\
\hline P- 89 & $\begin{array}{l}\text { no. 47-HW-302 } \\
\text { Phosphate rock, argillaceous; fos. col. } \\
\text { no. 47-HW-301 }\end{array}$ \\
\hline P- 88 & Phosphate rock \\
\hline P- 87 & Phosphate rock, argillaceous \\
\hline P- 86 & Phosphate rock, argillaceous \\
\hline $\begin{array}{l}P-85 \\
P-84\end{array}$ & Phosphate rock, argillaceous \\
\hline & Phosphate rock, argillaceous \\
\hline $\begin{array}{l}\text { P- } 83 \\
\text { P- } 82\end{array}$ & Phosphate rock, argillaceous \\
\hline $\begin{array}{l}-82 \\
P-81\end{array}$ & $\begin{array}{l}\text { Mudstone and phosphate rock } \\
\text { Mudstone, phosphatic }\end{array}$ \\
\hline P- 80 & Mudstone, phosphatic \\
\hline P- 79 & $\begin{array}{l}\text { Mudstone, calcareous; fos. col. no. } \\
47-\mathrm{HW}-300\end{array}$ \\
\hline P- 78 & Mudstone, phosphatic \\
\hline P- 77 & $\begin{array}{l}\text { Mudstone, phosphatic; fos. col. no. } \\
\text { 47-HW-299 }\end{array}$ \\
\hline P- 76 & Mudstone \\
\hline $\begin{array}{l}P-75 \\
P-74\end{array}$ & $\begin{array}{l}\text { Mudstone, phosphatic } \\
\text { Mudstone }\end{array}$ \\
\hline P- 73 & Mudstone; fos. col. no. 47-HW-298 \\
\hline P- 72 & Phosphate rock, argillaceous \\
\hline P- 71 & Mudstone \\
\hline P- 70 & Mudstone, phosphatic \\
\hline P- 69 & Mudstone, phosphatic and chert \\
\hline P- 68 & Mudstone, phosphatic \\
\hline P- 67 & Mudstone \\
\hline$P-66$ & Mudstone, cherty \\
\hline P- 65 & Mudstone \\
\hline P- & Mudstone and phosphate rock \\
\hline P- 63 & Mudstone and phosphate rock \\
\hline $\mathbf{P}-$ & Mudstone \\
\hline P- & Mudstone \\
\hline P- & Mudstone \\
\hline & Mudstone, phosphatic \\
\hline & $\begin{array}{l}\text { Mudstone, phosphatic; fos. col. no. } \\
\text { 47-HW-297 }\end{array}$ \\
\hline P- 57 & $\begin{array}{l}\text { Mudstone, phosphatic; fos. col. no. } \\
47-\mathrm{HW}-296\end{array}$ \\
\hline P- 56 & Mudstone; fos. col. no. 47-HW-295 \\
\hline
\end{tabular}

\begin{tabular}{|c|c|c|c|c|c|}
\hline $\begin{array}{l}\text { VEM-483-47 } \\
\text { VEM-482-47 } \\
\text { VEM-481-47 }\end{array}$ & $\begin{array}{l}0.4 \\
0.8 \\
0.8\end{array}$ & $\begin{array}{r}32.3 \\
32.4 \\
3.0\end{array}$ & $\begin{array}{l}2.2 \\
1.6 \\
4.2\end{array}$ & $\begin{array}{l}0.70 \\
1.49 \\
1.23\end{array}$ & $\begin{array}{r}6.48 \\
5.84 \\
10.54\end{array}$ \\
\hline VEM-480-47 & 0.6 & 19.9 & 5.2 & 1.60 & 13.54 \\
\hline VEM-479-47 & 0.6 & 20.4 & 5.5 & 1.53 & 15.76 \\
\hline $\begin{array}{l}\text { VEM-478-47 } \\
\text { VEM-477-47 } \\
\text { VEM-476-47 } \\
\text { VEM-475-47 } \\
\text { VEM-474-47 }\end{array}$ & $\begin{array}{l}1.0 \\
0.7 \\
0.7 \\
0.6 \\
0.8\end{array}$ & $\begin{array}{l}26.3 \\
23.9 \\
19.4 \\
19.2 \\
22.6\end{array}$ & $\begin{array}{l}3.2 \\
4.1 \\
5.5 \\
6.6 \\
4.7\end{array}$ & $\begin{array}{l}1.22 \\
1.48 \\
1.91 \\
2.15 \\
1.65\end{array}$ & $\begin{array}{l}16.42 \\
16.74 \\
11.18 \\
15.14 \\
14.42\end{array}$ \\
\hline $\begin{array}{l}\text { VEM-473-47 } \\
\text { VEM-472-47 } \\
\text { VEM-471-47 } \\
\text { VEM-358-47 }\end{array}$ & $\begin{array}{l}1.2 \\
1.0 \\
1.0 \\
1.2\end{array}$ & $\begin{array}{l}20.4 \\
16.6 \\
12.9 \\
15.6\end{array}$ & $\begin{array}{c}4.5 \\
-. \\
-. \\
-.\end{array}$ & $\begin{array}{c}1.59 \\
-- \\
-- \\
--\end{array}$ & $\begin{array}{c}14.64 \\
\ldots \\
\ldots \\
\ldots\end{array}$ \\
\hline VEM-357-47 & 1.5 & 2.7 & -- & -- & -- \\
\hline VEM-356-47 & 2.0 & 14.1 & $-\infty$ & -- & -- \\
\hline $\begin{array}{l}\text { VEM-355-47 } \\
\text { VEM-354-47 } \\
\text { VEM-353-4 } \\
\text { VEM-352-47 }\end{array}$ & $\begin{array}{l}0.8 \\
2.9 \\
1.7 \\
0.8\end{array}$ & $\begin{array}{r}15.5 \\
7.1 \\
10.7 \\
3.3\end{array}$ & $\begin{array}{l}-- \\
-- \\
-- \\
--\end{array}$ & $\begin{array}{l}-- \\
-- \\
-- \\
--\end{array}$ & $\begin{array}{l}-- \\
-- \\
--\end{array}$ \\
\hline $\begin{array}{l}\text { VEM-351-47 } \\
\text { VEM-350-47 } \\
\text { VEM-349-47 } \\
\text { VEM-348-47 } \\
\text { VEM-347-47 }\end{array}$ & $\begin{array}{l}1.6 \\
0.8 \\
1.2 \\
1.3 \\
0.8\end{array}$ & $\begin{array}{r}2.4 \\
18.5 \\
1.5 \\
8.2 \\
15.3\end{array}$ & $\begin{array}{l}-- \\
-- \\
-- \\
-- \\
--\end{array}$ & $\begin{array}{l}-- \\
-- \\
-- \\
--\end{array}$ & $\begin{array}{l}-- \\
-- \\
-- \\
-- \\
--\end{array}$ \\
\hline $\begin{array}{l}\text { VEM-346-47 } \\
\text { VEM-345-47 } \\
\text { RMC- } 90-47 \\
\text { RAW- } 51-47 \\
\text { RAW- } 50-47\end{array}$ & $\begin{array}{l}1.7 \\
2.4 \\
1.2 \\
1.1 \\
2.1\end{array}$ & $\begin{array}{l}8.0 \\
4.7 \\
3.2 \\
1.1 \\
7.6\end{array}$ & $\begin{array}{l}-- \\
-- \\
-- \\
-- \\
--\end{array}$ & $\begin{array}{l}-- \\
-- \\
-- \\
--\end{array}$ & $\begin{array}{l}-- \\
-- \\
-- \\
--\end{array}$ \\
\hline $\begin{array}{l}\text { RAW- } 27-47 \\
\text { RAW- } 26-47 \\
\text { RAW- } 25-47 \\
\text { RAG- } 15-47 \\
\text { RAG- } 14-47\end{array}$ & $\begin{array}{l}1.5 \\
0.9 \\
0.6 \\
4.82 \\
0.75\end{array}$ & $\begin{array}{r}12.6 \\
7.3 \\
7.5 \\
4.3 \\
16.6\end{array}$ & $\begin{array}{l}-- \\
-- \\
-- \\
-- \\
--\end{array}$ & $\begin{array}{l}-- \\
-- \\
-- \\
--\end{array}$ & $\begin{array}{l}-- \\
-- \\
-- \\
--\end{array}$ \\
\hline RAG- $13-47$ & 0.85 & 10.5 & -- & -- & - \\
\hline $\begin{array}{ll}\text { RAG- } & 12-47 \\
\text { RAG- } & 11-47\end{array}$ & $\begin{array}{l}0.30 \\
1.92\end{array}$ & $\begin{array}{r}15.6 \\
6.8\end{array}$ & -- & -- & -- \\
\hline
\end{tabular}

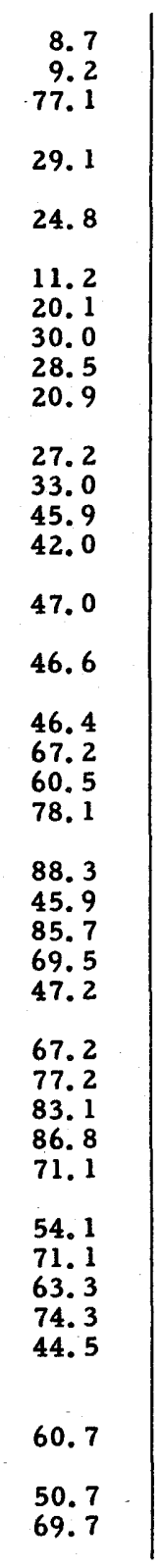

\begin{tabular}{|c|c|}
\hline $\begin{array}{l}24.5 \\
25.3 \\
26.1\end{array}$ & $\begin{array}{l}355.15 \\
381.07 \\
383.47\end{array}$ \\
\hline 26.7 & 395.41 \\
\hline 27.3 & 407.65 \\
\hline $\begin{array}{l}28.3 \\
29.0 \\
29.7 \\
30.3 \\
31.1\end{array}$ & $\begin{array}{l}433.95 \\
450.68 \\
464.26 \\
475.78 \\
493.86\end{array}$ \\
\hline $\begin{array}{l}32.3 \\
33.3 \\
34.3 \\
35.5\end{array}$ & $\begin{array}{l}518.34 \\
534.94 \\
547.84 \\
566.56\end{array}$ \\
\hline 37.0 & 570.61 \\
\hline 39.0 & 598.81 \\
\hline $\begin{array}{l}39.8 \\
42.7 \\
44.4 \\
45.2\end{array}$ & $\begin{array}{l}611.21 \\
631.80 \\
649.99 \\
652.63\end{array}$ \\
\hline $\begin{array}{l}46.8 \\
47.6 \\
48.8 \\
50.1 \\
50.9\end{array}$ & $\begin{array}{l}656.47 \\
671.27 \\
673.07 \\
683.73 \\
695.97\end{array}$ \\
\hline $\begin{array}{l}52.6 \\
55.0 \\
56.2 \\
57.3 \\
59.4\end{array}$ & $\begin{array}{l}709.57 \\
720.85 \\
724.69 \\
725.90 \\
741.86\end{array}$ \\
\hline $\begin{array}{l}60.9 \\
61.8 \\
62.4 \\
67.22 \\
67.97\end{array}$ & $\begin{array}{l}760.76 \\
767.33 \\
771.83 \\
792.56 \\
805.01\end{array}$ \\
\hline 68.82 & 813.93 \\
\hline $\begin{array}{l}69.12 \\
71.04\end{array}$ & $\begin{array}{l}818.61 \\
831.67\end{array}$ \\
\hline
\end{tabular}

${ }^{1}$ Fossil collection made by H. Wedow, Paleontology and Stratigraphy Branch, U. S. Geological Survey. 


\begin{tabular}{|c|c|c|c|c|c|c|c|c|c|c|}
\hline \multirow[b]{2}{*}{$\begin{array}{l}\text { Bed } \\
\text { no. }\end{array}$} & \multirow[b]{2}{*}{ Rock description } & \multirow{2}{*}{$\begin{array}{l}\text { Sample } \\
\text { no. }\end{array}$} & \multirow{2}{*}{$\begin{array}{l}\text { Thickness } \\
\text { (feet) }\end{array}$} & \multicolumn{5}{|c|}{ Chemical analyses (percent) } & \multirow{2}{*}{$\begin{array}{l}\text { Cumulative } \\
\text { thickness } \\
\text { (feet) }\end{array}$} & \multirow{2}{*}{$\begin{array}{l}\text { Thickness } x \\
\text { percent } \mathrm{P}_{2} \mathrm{O}_{5} \\
\text { (cumulative) }\end{array}$} \\
\hline & & & & $\mathrm{P}_{2} \mathrm{O}_{5}$ & $\mathrm{Al}_{2} \mathrm{O}_{3}$ & $\mathrm{Fe}_{2} \mathrm{O}_{3}$ & $\begin{array}{l}\text { Loss on } \\
\text { ignition }\end{array}$ & $\begin{array}{c}\text { Acid } \\
\text { insoluble }\end{array}$ & & \\
\hline P- 55 & $\begin{array}{l}\text { Mudstone, phosphatic; fos. col. no. } \\
47-\mathrm{HW}-294\end{array}$ & RAG- $10-47$ & 1.02 & 14.8 & -- & -- & -- & 46.5 & 72.06 & 846.76 \\
\hline $\begin{array}{l}P-54 \\
P-53\end{array}$ & $\begin{array}{l}\text { Mudstone } \\
\text { Mudstone, phosphatic, contains chert }\end{array}$ & RAG- $\quad 9-47$ & 2.62 & 5.5 & -- & -- & -- & 70.8 & 74.68 & 861.17 \\
\hline $\begin{array}{l}P-52 \\
P-51\end{array}$ & $\begin{array}{l}\text { nodules } \\
\text { Mudstone } \\
\text { Phosphate rock, argillaceous }\end{array}$ & $\begin{array}{lr}\text { RAG - } & 8-47 \\
\text { RAG - } & 7-47 \\
\text { RAH- } 216-47\end{array}$ & $\begin{array}{l}0.62 \\
3.3 \\
0.4\end{array}$ & $\begin{array}{r}11.8 \\
1.3 \\
23.0\end{array}$ & -- & $\begin{array}{l}-- \\
-- \\
--\end{array}$ & $\begin{array}{l}-- \\
-- \\
--\end{array}$ & $\begin{array}{l}58.9 \\
86.8 \\
32.0\end{array}$ & $\begin{array}{l}75.30 \\
78.60 \\
79.00\end{array}$ & $\begin{array}{l}868.49 \\
872.78 \\
881.98\end{array}$ \\
\hline $\begin{array}{l}P-50 \\
P-49 \\
P-48 \\
P-47 \\
P-46\end{array}$ & $\begin{array}{l}\text { Mudstone } \\
\text { Mudstone } \\
\text { Mudstone } \\
\text { Phosphate rock } \\
\text { Mudstone }\end{array}$ & $\begin{array}{l}\text { RAH- } 215-47 \\
\text { RAH- 214-47 } \\
\text { RAH- } 199-47 \\
\text { RAH- } 198-47 \\
\text { RAH- } 197-47\end{array}$ & $\begin{array}{l}1.9 \\
1.3 \\
0.9 \\
0.3 \\
1.0\end{array}$ & $\begin{array}{r}1.8 \\
0.6 \\
0.7 \\
30.0 \\
3.3\end{array}$ & $\begin{array}{l}-- \\
-- \\
-- \\
-- \\
--\end{array}$ & $\begin{array}{l}-- \\
-- \\
-- \\
--\end{array}$ & $\begin{array}{l}-- \\
-- \\
-- \\
--\end{array}$ & $\begin{array}{l}84.2 \\
87.3 \\
88.5 \\
18.9 \\
77.9\end{array}$ & $\begin{array}{l}80.90 \\
82.20 \\
83.10 \\
83.40 \\
84.40\end{array}$ & $\begin{array}{l}885.40 \\
886.18 \\
886.81 \\
895.81 \\
899.11\end{array}$ \\
\hline $\begin{array}{l}P-45 \\
P-44 \\
P-43 \\
P-42 \\
P-41\end{array}$ & $\begin{array}{l}\text { Mudstone } \\
\text { Mudstone; fos. col. no. } 47-\mathrm{HW}-293 \\
\text { Mudstone; fos. col. no. } 47-\mathrm{HW}-292 \\
\text { Mudstone, phosphatic } \\
\text { Mudstone }\end{array}$ & $\begin{array}{l}\text { RAH- } 196-47 \\
\text { RAH- } 195-47 \\
\text { RAH- } 194-47 \\
\text { RAH- } 193-47 \\
\text { RAH- } 192-47\end{array}$ & $\begin{array}{l}3.0 \\
0.5 \\
1.9 \\
0.9 \\
1.4\end{array}$ & $\begin{array}{l}2.4 \\
3.2 \\
0.8 \\
9.8 \\
1.6\end{array}$ & $\begin{array}{l}-- \\
-- \\
-- \\
-- \\
--\end{array}$ & $\begin{array}{l}-- \\
-- \\
-- \\
-- \\
--\end{array}$ & $\begin{array}{l}-- \\
-- \\
-- \\
-- \\
--\end{array}$ & $\begin{array}{l}84.1 \\
80.8 \\
85.6 \\
62.0 \\
84.6\end{array}$ & $\begin{array}{l}87.40 \\
87.90 \\
89.80 \\
90.70 \\
92.10\end{array}$ & $\begin{array}{l}906.31 \\
907.91 \\
909.43 \\
918.25 \\
920.49\end{array}$ \\
\hline & $\begin{array}{l}\text { Mudstone, phosphatic; fos. col. no. } \\
\text { 47-HW-291 }\end{array}$ & RAH- $191-47$ & 0.7 & 14.7 & -- & -- & -- & 46.5 & 92.80 & 930.78 \\
\hline $\begin{array}{l}P=39 \\
P-38 \\
P=37 \\
P=36\end{array}$ & $\begin{array}{l}\text { Phosphate rock, argillaceous; fos. } \\
\text { col. no. } 47-\mathrm{HW}-291 \\
\text { Mudstone } \\
\text { Mudstone, phosphatic } \\
\text { Mudstone }\end{array}$ & $\begin{array}{l}\text { RAH- } 190-47 \\
\text { RAG- } 24-47 \\
\text { RAG- } 23-47 \\
\text { RAG- } 22-47\end{array}$ & $\begin{array}{l}0.6 \\
2.64 \\
1.48 \\
1.91\end{array}$ & \begin{tabular}{r|}
19.7 \\
4.4 \\
11.9 \\
7.0
\end{tabular} & $\begin{array}{l}-- \\
-- \\
--\end{array}$ & $\begin{array}{l}-- \\
-- \\
--\end{array}$ & $\begin{array}{l}-- \\
-- \\
--\end{array}$ & $\begin{array}{l}39.0 \\
79.9 \\
61.9 \\
73.2\end{array}$ & $\begin{array}{l}93.40 \\
96.04 \\
97.52 \\
99.43\end{array}$ & $\begin{array}{l}942.60 \\
954.22 \\
971.83 \\
985.20\end{array}$ \\
\hline $\begin{array}{l}P-35 \\
P-34 \\
P-33\end{array}$ & $\begin{array}{l}\text { Mudstone; fos. col. no. 47-HW-290 } \\
\text { Phosphate rock and phosphatic mudstone }\end{array}$ & $\begin{array}{l}\text { RAG- } 21-47 \\
\text { RAG- } 20-47\end{array}$ & $\begin{array}{l}3.0 \\
0.95\end{array}$ & $\begin{array}{r}1.1 \\
22.7\end{array}$ & -- & -- & -- & $\begin{array}{l}88.1 \\
31.0\end{array}$ & $\begin{array}{l}102.43 \\
103.38\end{array}$ & $\begin{array}{r}988.50 \\
1,010.06\end{array}$ \\
\hline $\begin{array}{l}P=33 \\
P=32 \\
P-31\end{array}$ & $\begin{array}{l}\text { Mudstone, phosphatic; tos. col. no. } \\
47-\text { HW-289 } \\
\text { Phosphate rock } \\
\text { Mudstone }\end{array}$ & $\begin{array}{l}\text { RAG- } 19-47 \\
\text { RAG- } 18-47 \\
\text { RAG- } 17-47\end{array}$ & $\begin{array}{l}1.16 \\
0.45 \\
1.71\end{array}$ & $\begin{array}{r}8.0 \\
32.0 \\
4.3\end{array}$ & $\begin{array}{l}-- \\
-- \\
--\end{array}$ & $\begin{array}{l}-- \\
-- \\
--\end{array}$ & $\ddot{--}$ & $\begin{array}{r}70.8 \\
8.2 \\
78.2\end{array}$ & $\begin{array}{l}104.54 \\
104.99 \\
106.70\end{array}$ & $\begin{array}{l}1,019.34 \\
1,033.74 \\
1,041.10\end{array}$ \\
\hline $\begin{array}{l}P-30 \\
P=29 \\
P=28 \\
P=27 \\
P-26\end{array}$ & $\begin{array}{l}\text { Mudstone, phosphatic } \\
\text { Mudstone } \\
\text { Mudstone, phosphatic } \\
\text { Mudstone, phosphatic } \\
\text { Mudstone, phosphatic }\end{array}$ & $\begin{array}{l}\text { VEM-507-47 } \\
\text { VEM-506-47 } \\
\text { VEM-505-47 } \\
\text { VEM-504-47 } \\
\text { VEM-503-47 }\end{array}$ & $\begin{array}{l}1.9 \\
0.6 \\
1.4 \\
1.6 \\
0.6\end{array}$ & $\begin{array}{r}11.6 \\
3.0 \\
13.6 \\
12.4 \\
9.4\end{array}$ & $\begin{array}{l}-- \\
-- \\
-- \\
--\end{array}$ & $\begin{array}{l}-- \\
-- \\
-- \\
--\end{array}$ & $\begin{array}{l}-- \\
-- \\
-- \\
--\end{array}$ & $\begin{array}{l}52.3 \\
78.6 \\
43.9 \\
48.8 \\
57.4\end{array}$ & $\begin{array}{l}108.60 \\
109.20 \\
110.60 \\
112.20 \\
112.80\end{array}$ & $\begin{array}{l}1,063.14 \\
1,064.94 \\
1,083.98 \\
1,103.82 \\
1,169.46\end{array}$ \\
\hline $\begin{array}{l}P-25 \\
P-24 \\
P-23 \\
P=22 \\
P-21\end{array}$ & $\begin{array}{l}\text { Mudstone, phosphatic } \\
\text { Mudstone } \\
\text { Mudstone; fos. col. no. } 47-\mathrm{HW}-306 \\
\text { Mudstone } \\
\text { Mudstone; fos. col. no. } 47-\mathrm{HW}-305\end{array}$ & $\begin{array}{l}\text { VEM-502-47 } \\
\text { VEM-501-47 } \\
\text { VEM-500-47 } \\
\text { VEM-499-47 } \\
\text { VEM-498-47 }\end{array}$ & $\begin{array}{l}0.8 \\
1.6 \\
2.8 \\
0.8 \\
1.1\end{array}$ & $\begin{array}{l}9.0 \\
5.0 \\
2.5 \\
4.7 \\
3.9\end{array}$ & $\begin{array}{l}-- \\
-- \\
-- \\
-- \\
--\end{array}$ & $\begin{array}{l}-- \\
-- \\
-- \\
-- \\
--\end{array}$ & $\begin{array}{l}-- \\
-- \\
-- \\
-- \\
--\end{array}$ & $\begin{array}{l}58.2 \\
72.0 \\
79.4 \\
72.6 \\
77.1\end{array}$ & $\begin{array}{l}113.60 \\
115.20 \\
118.00 \\
118.80 \\
119.90\end{array}$ & $\begin{array}{l}1,116.66 \\
1,124.66 \\
1,131.66 \\
1,135.42 \\
1,139.71\end{array}$ \\
\hline $\begin{array}{l}P=20 \\
P=19\end{array}$ & $\begin{array}{l}\text { Mudstone } \\
\text { Mudstone }\end{array}$ & $\begin{array}{l}\text { VEM-497-47 } \\
\text { VEM-496-47 }\end{array}$ & $\begin{array}{l}0.9 \\
1.5\end{array}$ & $\begin{array}{l}7.4 \\
1.2\end{array}$ & -- & -- & -- & $\begin{array}{l}65.0 \\
86.0\end{array}$ & $\begin{array}{l}120.80 \\
122.30\end{array}$ & $\begin{array}{l}1,146.37 \\
1,148.17\end{array}$ \\
\hline
\end{tabular}




\begin{tabular}{|c|c|c|c|c|c|c|c|c|c|c|}
\hline $\begin{array}{l}P-18 \\
P-17 \\
P-16 \\
P-15 \\
P-14\end{array}$ & $\begin{array}{l}\text { Mudstone, phosphatic } \\
\text { Mudstone, phosphatic } \\
\text { Mudstone } \\
\text { Mudstone, phosphatic } \\
\text { Mudstone, phosphatic }\end{array}$ & $\begin{array}{l}\text { VEM-495-47 } \\
\text { VEM-494-47 } \\
\text { RMC-111-47 } \\
\text { RMC-110-47 } \\
\text { RMC-109-47 }\end{array}$ & $\begin{array}{l}1.6 \\
1.1 \\
1.0 \\
1.4 \\
1.4\end{array}$ & $\begin{array}{r}11.3 \\
8.8 \\
6.4 \\
12.4 \\
14.8\end{array}$ & $\begin{array}{l}-- \\
-- \\
-- \\
--\end{array}$ & $\begin{array}{l}-- \\
-- \\
-- \\
--\end{array}$ & $\begin{array}{l}-- \\
-- \\
-- \\
--\end{array}$ & $\begin{array}{l}55.7 \\
61.9 \\
71.8 \\
52.8 \\
46.6\end{array}$ & $\begin{array}{l}123.90 \\
125.00 \\
126.00 \\
127.40 \\
128.80\end{array}$ & $\begin{array}{l}1,166.25 \\
1,175.93 \\
1,182.33 \\
1,199.69 \\
1,220.41\end{array}$ \\
\hline $\begin{array}{l}P-13 \\
P-12 \\
P-11 \\
P-10 \\
P-9\end{array}$ & $\begin{array}{l}\text { Mudstone } \\
\text { Mudstone, phosphatic } \\
\text { Phosphate rock, argillaceous } \\
\text { Mudstone and phosphate rock } \\
\text { Mudstone, phosphatic }\end{array}$ & $\begin{array}{l}\text { RMC-108-47 } \\
\text { RMC-107-47 } \\
\text { RMC-106-47 } \\
\text { RMC-105-47 } \\
\text { VEM-517-47 }\end{array}$ & $\begin{array}{l}0.9 \\
1.7 \\
1.5 \\
1.3 \\
0.7\end{array}$ & $\begin{array}{r}3.0 \\
14.3 \\
16.8 \\
14.8 \\
15.5\end{array}$ & $\begin{array}{l}-- \\
-- \\
--\end{array}$ & $\begin{array}{l}-- \\
-- \\
--\end{array}$ & $\begin{array}{l}-- \\
-- \\
-- \\
--\end{array}$ & $\begin{array}{l}82.9 \\
48.3 \\
40.0 \\
47.6 \\
45.3\end{array}$ & $\begin{array}{l}129.70 \\
131.40 \\
132.90 \\
134.20 \\
134.90\end{array}$ & $\begin{array}{l}1,223.11 \\
1,247.42 \\
1,272.62 \\
1,291.86 \\
1,302.71\end{array}$ \\
\hline $\begin{array}{ll}\text { P- } & 8 \\
P- & 7 \\
P- & 6 \\
P- & 5 \\
P- & 4\end{array}$ & $\begin{array}{l}\text { Phosphate rock, argillaceous } \\
\text { Phosphate rock } \\
\text { Mudstone, phosphatic } \\
\text { Mudstone, phosphatic } \\
\text { Mudstone }\end{array}$ & $\begin{array}{l}\text { VEM-516-47 } \\
\text { VEM-515-47 } \\
\text { VEM-514-47 } \\
\text { VEM-51 }-513-47 \\
\text { VEM-51 } 12-47\end{array}$ & $\begin{array}{l}2.0 \\
1.6 \\
1.9 \\
1.0 \\
1.2\end{array}$ & $\begin{aligned} 21.2 \\
28.5 \\
13.2 \\
11.5 \\
5.6\end{aligned}$ & $\begin{array}{l}-- \\
-- \\
-- \\
--\end{array}$ & $\begin{array}{l}-- \\
-- \\
-- \\
--\end{array}$ & $\begin{array}{l}-- \\
-- \\
-- \\
--\end{array}$ & $\begin{array}{l}27.5 \\
15.8 \\
50.2 \\
55.0 \\
68.8\end{array}$ & $\begin{array}{l}136.90 \\
138.50 \\
140.40 \\
141.40 \\
142.60\end{array}$ & $\begin{array}{l}1,345.11 \\
1,390.71 \\
1,415.79 \\
1,427.29 \\
1,434.01\end{array}$ \\
\hline $\begin{array}{ll}P- & 3 \\
P- & 2 \\
P- & 1\end{array}$ & $\begin{array}{l}\text { Mudstone } \\
\text { Mudstone, phosphatic } \\
\text { Phosphate rock }\end{array}$ & $\begin{array}{l}\text { VEM-511-47 } \\
\text { VEM-510-47 } \\
\text { VEM-509-47 }\end{array}$ & $\begin{array}{l}1.8 \\
0.4 \\
0.25\end{array}$ & $\begin{array}{r}1.4 \\
9.5 \\
35.7\end{array}$ & $\begin{array}{ll}-- \\
--\end{array}$ & $\begin{array}{l}-- \\
-- \\
--\end{array}$ & $\overline{--}$ & $\begin{array}{r}75.0 \\
56.5 \\
4.8\end{array}$ & $\begin{array}{l}144.40 \\
144.80 \\
145.05\end{array}$ & $\begin{array}{l}1,436.53 \\
1,440.33 \\
1,449.26\end{array}$ \\
\hline & The lower phosphate bed appears & nt due to $\mathrm{f}$ & & & & & & & & \\
\hline \multicolumn{11}{|c|}{ Wells formation } \\
\hline $\begin{array}{l}\mathrm{Cw}-1 \\
\mathrm{Cw}-2 \\
\mathrm{Cw}-3\end{array}$ & $\begin{array}{l}\text { Dolomite; fos. col. no. } 47-\mathrm{HW}-304 \\
\text { Clay and phosphate rock } \\
\text { Dolomite }\end{array}$ & $\begin{array}{c}\text { VEM- } 508-47 \\
-- \\
--\end{array}$ & $\begin{array}{l}3.2 \\
0.1 \\
0.2\end{array}$ & $\begin{array}{ll}1.1 \\
-- \\
--\end{array}$ & $\begin{array}{ll}-- \\
--\end{array}$ & $\overline{--}$ & -- & $\begin{array}{l}7.3 \\
-- \\
-- \\
\end{array}$ & $\begin{array}{l}3.2 \\
3.3 \\
3.5\end{array}$ & $\begin{array}{l}3.52 \\
-- \\
--\end{array}$ \\
\hline
\end{tabular}


SPECTROGRAPHIC ANALYSES - JOHNSON CREEK, IDAHO. LOT NO. 1209.

Semi-quantitative analyses of selected samples of the phosphatic shale member of the Phosphoria formation, Johnson Creek, Idaho (see immediately preceding pages for location of section, thickness and description of strata, and chemical analyses of samples), made by the U. S. Geological Survey Laboratory, Geochemistry and Petrology Branch, Washington, D. C. In addition to the elements listed in the table below, Sb, As, Be, Bi, Cs, Co, Cb, Dy, Sm, Sc, Ta, Te, Tb, Tl, Tm, W, and $\mathrm{Yb}$ were looked for in all samples but were not detected.

Explanation of symbols

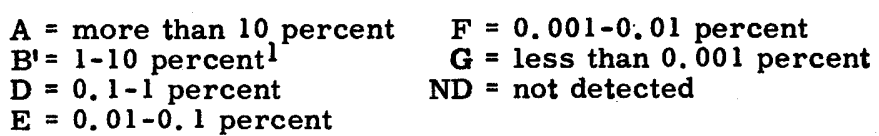

$E=0.01-0.1$ percent

$F=0.001-0.01$ percent

$\mathbf{G}=$ less than 0.001 percent

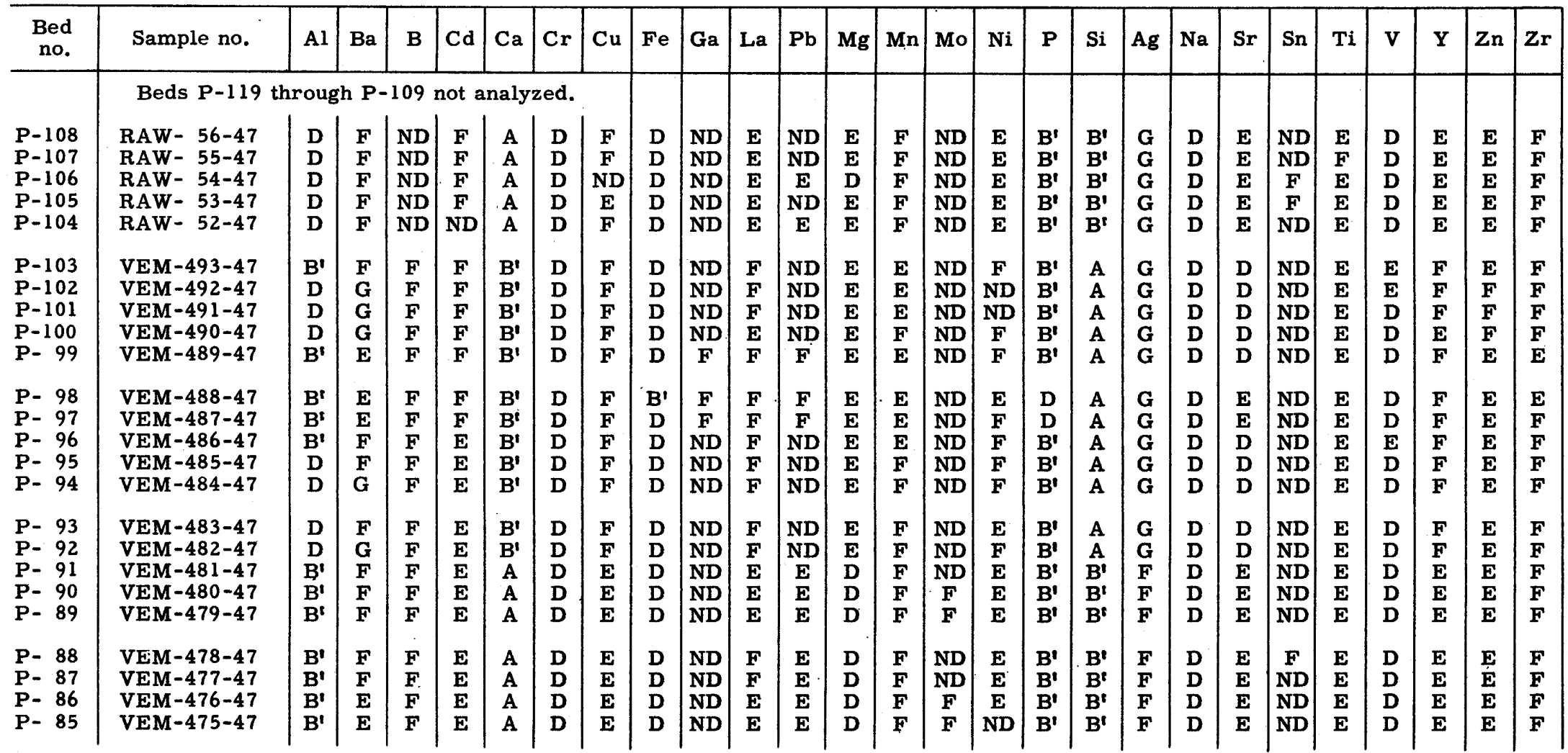




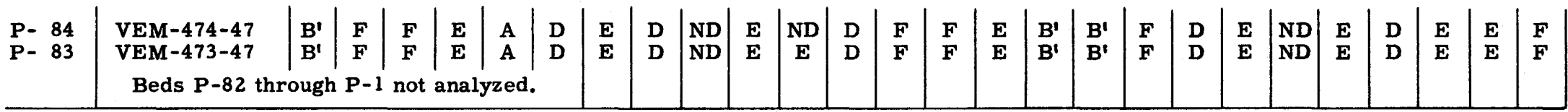

${ }^{1} \mathrm{~B}^{\prime}$ is equivalent to $\mathrm{B}$ and $\mathrm{C}$ of Bureau of Mines analyses as recorded in other reports. 


\begin{tabular}{|c|c|c|c|c|c|c|c|c|c|c|c|}
\hline $\begin{array}{l}P-158 \\
P-157\end{array}$ & Mudstone, phosphatic & RAG- $116-47$ & 0.98 & 9.0 & 11. & 3.29 & -- & 3.34 & 61.8 & 24.61 & 200.39 \\
\hline & phosphate rock & RAG- $115-47$ & 0.74 & 24.8 & 5.7 & 2.15 & 2.53 & 3.46 & 27.4 & 25.35 & 218.74 \\
\hline $\begin{array}{l}P-156 \\
P-155\end{array}$ & $\begin{array}{l}\text { Phosphate rock } \\
\text { Mudstone, phosphatic and }\end{array}$ & RAG-114-47 & 0.6 & 32.5 & 2.2 & 1.10 & -- & 2.84 & 12.9 & 25.95 & 238.24 \\
\hline$P-154$ & $\begin{array}{l}\text { phosphate rock } \\
\text { Phosphate rock }\end{array}$ & $\begin{array}{l}\text { RAG- } 113-47 \\
\text { RAG- } 112-47\end{array}$ & $\begin{array}{l}1.13 \\
0.8\end{array}$ & $\begin{array}{l}20.3 \\
32.0\end{array}$ & $\begin{array}{l}6.7 \\
2.3\end{array}$ & $\begin{array}{l}2.16 \\
1.05\end{array}$ & $\begin{array}{ll}-- \\
--\end{array}$ & $\begin{array}{l}4.44 \\
2.90\end{array}$ & $\begin{array}{l}36.1 \\
13.1\end{array}$ & $\begin{array}{l}27.08 \\
27.88\end{array}$ & $\begin{array}{l}261.18 \\
286.78\end{array}$ \\
\hline $\begin{array}{l}\text { P- } 153 \\
\text { P-152 } \\
--\end{array}$ & $\begin{array}{l}\text { Mudstone, phosphatic and } \\
\text { phosphate rock } \\
\text { Phosphate rock } \\
\text { Phosphate rock }\end{array}$ & $\begin{array}{l}\text { RAG- } 111-47 \\
\text { RAW-104-47 } \\
\text { RAG- } 110-47\end{array}$ & $\begin{array}{l}0.79 \\
0.8 \\
(0.82)\end{array}$ & $\begin{array}{l}12.6 \\
34.4 \\
35.3\end{array}$ & $\begin{array}{l}9.2 \\
1.8 \\
--\end{array}$ & $\begin{array}{l}2.76 \\
0.74\end{array}$ & $\begin{array}{l}-- \\
-- \\
--\end{array}$ & $\begin{array}{l}4.82 \\
3.20 \\
---\end{array}$ & $\begin{array}{r}54.0 \\
7.2 \\
6.0\end{array}$ & $\begin{array}{l}28.67 \\
29.47 \\
--\end{array}$ & $\begin{array}{l}296.74 \\
324.26 \\
--\end{array}$ \\
\hline $\begin{array}{c}P-151 \\
--\end{array}$ & $\begin{array}{l}\text { Phosphate rock and phosphatic } \\
\text { mudstone } \\
\text { Phosphate rock }\end{array}$ & $\begin{array}{l}\text { RMC }-96-47 \\
\text { RAG- } 109-47\end{array}$ & $\begin{array}{l}0.5 \\
(0.45)\end{array}$ & $\begin{array}{l}26.4 \\
32.0\end{array}$ & 4.5 & $\begin{array}{l}1.48 \\
--\end{array}$ & -- & 5.56 & $\begin{array}{l}22.6 \\
14.4\end{array}$ & 29.97 & $\begin{array}{c}337.46 \\
--\end{array}$ \\
\hline & RAG-109-47 and RAG-110-47 a & ogged as equiv & nt to $\mathrm{RN}$ & $-96-47$ & and $R$. & $w-104$ & & & & & \\
\hline$P-150$ & $\begin{array}{l}\text { Phosphate rock and argillaceous } \\
\text { phosphate rock }\end{array}$ & RMC - 95-47 & 0.7 & 30.9 & 2.7 & 0.98 & -- & 4.16 & 14.6 & 30.67 & 359.09 \\
\hline $\begin{array}{l}P-149 \\
P-148\end{array}$ & $\begin{array}{l}\text { Phosphate rock } \\
\text { Mudstone, phosphatic; fos. col. }\end{array}$ & RMC- $94-47$ & 0.5 & 30.4 & 2.6 & 1.02 & -- & 4.06 & 15.7 & 31.17 & 374.29 \\
\hline $\begin{array}{l}P-147 \\
P-146\end{array}$ & $\begin{array}{l}\text { no. } 47-\mathrm{HW}-280 \\
\text { Mudstone } \\
\text { Mudstone, phosphatic }\end{array}$ & $\begin{array}{l}\text { RMC- } 93-47 \\
\text { RMC- } 92-47 \\
\text { RMC- } 91-47\end{array}$ & $\begin{array}{l}0.5 \\
0.5 \\
0.7\end{array}$ & $\begin{array}{r}11.8 \\
5.2 \\
10.5\end{array}$ & $\begin{aligned} 8.3 \\
11 . \\
9.2\end{aligned}$ & $\begin{array}{l}2.71 \\
3.33 \\
2.60\end{array}$ & $\begin{array}{l}-- \\
-- \\
--\end{array}$ & $\begin{array}{l}6.32 \\
4.64 \\
5.02\end{array}$ & $\begin{array}{l}57.1 \\
73.5 \\
47.1\end{array}$ & $\begin{array}{l}31.67 \\
32.17 \\
32.87\end{array}$ & $\begin{array}{l}380.19 \\
382.79 \\
390.14\end{array}$ \\
\hline$P-145$ & $\begin{array}{l}\text { Phosphate rock and phosphatic } \\
\text { mudstone }\end{array}$ & VEM-608-47 & 0.7 & 27.6 & 3.7 & 1.27 & -- & 5.52 & 20.0 & 33.57 & 409.46 \\
\hline P-144 & $\begin{array}{l}\text { Phosphate rock and phosphatic } \\
\text { mudstone }\end{array}$ & & 1.7 & & 6.6 & 2.31 & -- & 5.80 & 38.9 & 35.27 & 441.42 \\
\hline $\begin{array}{l}P-143 \\
P-142\end{array}$ & $\begin{array}{l}\text { Mudstone } \\
\text { Phosphate rock and phosphatic }\end{array}$ & VEM $-606-47$ & 0.8 & 6.8 & 11. & 3.90 & -- & 8.74 & 63.3 & 36.07 & 446.86 \\
\hline$P-141$ & $\begin{array}{l}\text { mudstone } \\
\text { Phosphate rock }\end{array}$ & $\begin{array}{l}\text { VEM }-605-47 \\
\text { VEM }-604-47\end{array}$ & $\begin{array}{l}0.6 \\
0.6\end{array}$ & $\begin{array}{l}27.1 \\
33.9\end{array}$ & $\begin{array}{l}3.8 \\
1.4\end{array}$ & $\begin{array}{l}1.32 \\
0.58\end{array}$ & $\overline{--}$ & $\begin{array}{l}5.92 \\
5.14\end{array}$ & $\begin{array}{r}20.5 \\
8.0\end{array}$ & $\begin{array}{l}36.67 \\
37.27\end{array}$ & $\begin{array}{l}463.12 \\
483.46\end{array}$ \\
\hline $\begin{array}{l}P-140 \\
P-139 \\
P-138 \\
P-137\end{array}$ & $\begin{array}{l}\text { Phosphate rock } \\
\text { Phosphate rock } \\
\text { Phosphate rock } \\
\text { Phosphate rock, argillaceous }\end{array}$ & $\begin{array}{l}\text { VEM- }-603-47 \\
\text { VEM-602-47 } \\
\text { RMC-132-47 } \\
\text { RMC- } 131-47\end{array}$ & $\begin{array}{l}0.8 \\
1.1 \\
0.5 \\
0.7\end{array}$ & $\begin{array}{l}31.1 \\
30.8 \\
31.6 \\
23.4\end{array}$ & $\begin{array}{l}2.2 \\
2.6 \\
2.3 \\
5.3\end{array}$ & $\begin{array}{l}0.88 \\
1.06 \\
0.84 \\
2.28\end{array}$ & $\begin{array}{l}-- \\
-- \\
--\end{array}$ & $\begin{array}{r}12.48 \\
6.06 \\
5.72 \\
9.34\end{array}$ & $\begin{array}{l}11.2 \\
12.0 \\
11.5 \\
24.7\end{array}$ & $\begin{array}{l}38.07 \\
39.77 \\
40.27 \\
40.97\end{array}$ & $\begin{array}{l}508.34 \\
560.70 \\
576.50 \\
592.88\end{array}$ \\
\hline$P-136$ & $\begin{array}{l}\text { Phosphate rock, argillace ous, } \\
\text { calcareous }\end{array}$ & RMC $-130-47$ & 0.5 & 15.6 & 7.8 & 2.61 & -- & 15.90 & 35.4 & 41.47 & 600.68 \\
\hline$P-135$ & $\begin{array}{l}\text { Phosphate rock, argillaceous, } \\
\text { calcareous }\end{array}$ & RMC $-129-47$ & 1.0 & 18.4 & 6.1 & 2.14 & -- & 14.28 & 30.9 & 42.47 & 619.08 \\
\hline P-134 & $\begin{array}{l}\text { Phosphate rock and argillaceous } \\
\text { phosphate rock }\end{array}$ & RMC $-128-47$ & 0.8 & 22.2 & 5.0 & 1.89 & - & 12.86 & 24.5 & 43.27 & 636.84 \\
\hline $\begin{array}{l}P-133 \\
P-132\end{array}$ & $\begin{array}{l}\text { Phosphate rock } \\
\text { Phosphate rock }\end{array}$ & $\begin{array}{l}\text { RMC }-127-47 \\
\text { RMC-126-47 }\end{array}$ & $\begin{array}{l}0.8 \\
1.1\end{array}$ & $\begin{array}{l}25.8 \\
27.6\end{array}$ & $\begin{array}{l}3.4 \\
2.7\end{array}$ & $\begin{array}{l}1.48 \\
1.3\end{array}$ & -- & $\begin{array}{r}13.88 \\
9.48\end{array}$ & $\begin{array}{l}16.1 \\
14.6\end{array}$ & $\begin{array}{l}44.07 \\
45.17\end{array}$ & $\begin{array}{l}657.48 \\
687.84\end{array}$ \\
\hline$P-131$ & $\begin{array}{l}\text { Mudstone, phosphatic; fos. col. } \\
\text { no. 47-HW-279 }\end{array}$ & RMC-125-47 & 0.7 & 23.5 & 5.2 & 2.57 & -- & 9.78 & 25.2 & 45.87 & 704.29 \\
\hline
\end{tabular}

${ }^{1}$ Fossil collection made by H. Wedow, Paleontology and Stratigraphy Branch, U. S. Geological Survey. 


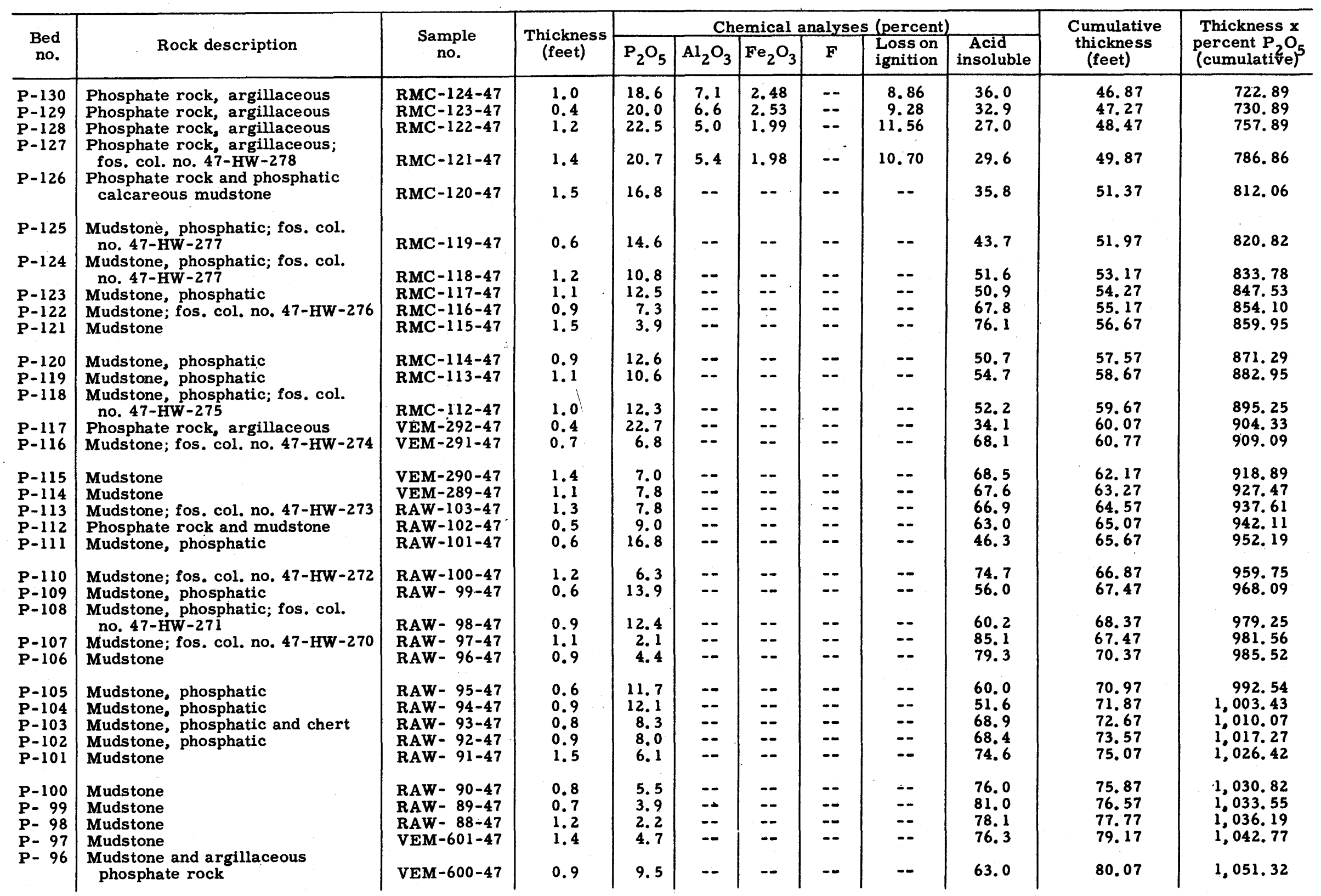




\begin{tabular}{|c|c|}
\hline $\begin{array}{l}P-95 \\
P=94 \\
P=93 \\
P=92 \\
P-91\end{array}$ & $\begin{array}{l}\text { Phosphate rock, argillaceous } \\
\text { Mudstone, phosphatic } \\
\text { Mudstone, phosphatic } \\
\text { Mudstone } \\
\text { Mudstone }\end{array}$ \\
\hline$P-90$ & $\begin{array}{l}\text { Phosphate rock, mudstone, and } \\
\text { phosphatic mudstone }\end{array}$ \\
\hline & $\begin{array}{l}\text { Mudstone, phosphatic } \\
\text { Mudstone }\end{array}$ \\
\hline D $\quad 27$ & $\begin{array}{l}\text { Phosphate rock, mudstone, and } \\
\text { phosphatic mudstone }\end{array}$ \\
\hline$P-86$ & Mudstone; fos. col. no. $47-\mathrm{HW}-269$ \\
\hline $\begin{array}{l}\text { P- } 85 \\
\text { P- } 84\end{array}$ & $\begin{array}{l}\text { Mudstone, phosphatic } \\
\text { Mudstone and argillaceous } \\
\text { phosphate rock }\end{array}$ \\
\hline P- & $\begin{array}{l}\text { Phosphate rock and phosphatic } \\
\text { mudstone }\end{array}$ \\
\hline $\begin{array}{l}P-82 \\
P-81\end{array}$ & $\begin{array}{l}\text { Mudstone, phosphatic } \\
\text { Mudstone, phosphatic and } \\
\text { mudstone; fos. col. no. } \\
\text { 47-HW-268 }\end{array}$ \\
\hline & $\begin{array}{l}\text { Phosphate rock, argillaceous, } \\
\text { and phosphatic mudstone }\end{array}$ \\
\hline $\begin{array}{l}P-79 \\
P-78 \\
P-77 \\
P-76\end{array}$ & $\begin{array}{l}\text { Mudstone; fos. col, no. } 47-\mathrm{HW}-267 \\
\text { Mudstone and phosphate rock } \\
\text { Mudstone } \\
\text { Mudstone }\end{array}$ \\
\hline $\begin{array}{l}P-75 \\
P-74 \\
P-73 \\
P-72 \\
P-71\end{array}$ & $\begin{array}{l}\text { Mudstone } \\
\text { Phosphate rock, argillaceous } \\
\text { Mudstone } \\
\text { Mudstone and phosphate rock } \\
\text { Mudstone }\end{array}$ \\
\hline $\begin{array}{l}P-70 \\
P-69 \\
P-68 \\
P-67 \\
P-66\end{array}$ & $\begin{array}{l}\text { Phosphate rock } \\
\text { Mudstone } \\
\text { Phosphate rock, argillaceous } \\
\text { Mudstone } \\
\text { Phosphate rock and mudstone }\end{array}$ \\
\hline $\begin{array}{l}P-65 \\
P-64 \\
P-63 \\
P-62 \\
P-61\end{array}$ & $\begin{array}{l}\text { Mudstone } \\
\text { Mudstone, phosphatic } \\
\text { Phosphate rock, argillaceous } \\
\text { Mudstone, phosphatic } \\
\text { Mudstone }\end{array}$ \\
\hline $\begin{array}{l}P=60 \\
P=59 \\
P=58\end{array}$ & $\begin{array}{l}\text { Phosphate rock, argillaceous } \\
\text { Mudstone, phosphatic } \\
\text { Phosphate rock, argillaceous }\end{array}$ \\
\hline
\end{tabular}

VEM-599-47
VEM-598-47
VEM-597-47
VEM-596-47
VEM-595-47

VEM-594-47
VEM-593-47
VEM-592-47
VEM-591-47
VEM-590-47
VEM-589-47
VEM-588-47
VEM-587-47
VEM-586-47
VE
VEM-585-47
VEM-564-47
VEM-562-47
VEM-565-47
VEM-584-47
VEM-583-47
VEM-5M-567-47
VEM-582-47
VEM-581-47
VEM-580-47
VEM-579-47
VEM-578-47
VEM-577-47
VEM-576-47
VEM-575-47
VEM-574-47
VEM-573-47
VEM-572-47
VEM-571-47
VEM-570-47

VEM

\begin{tabular}{|c|c|}
\hline $\begin{array}{l}0.5 \\
0.8 \\
0.7 \\
0.6 \\
0.4\end{array}$ & $\begin{array}{r}22.3 \\
9.6 \\
8.0 \\
5.8 \\
3.3\end{array}$ \\
\hline $\begin{array}{l}0.9 \\
0.8 \\
2.8\end{array}$ & $\begin{array}{l}8.3 \\
8.4 \\
5.1\end{array}$ \\
\hline $\begin{array}{l}0.9 \\
1.4\end{array}$ & $\begin{array}{r}15.0 \\
3.5\end{array}$ \\
\hline 1.4 & 9.1 \\
\hline 1.4 & 7.2 \\
\hline $\begin{array}{l}0.6 \\
0.5\end{array}$ & $\begin{array}{r}14.6 \\
8.5\end{array}$ \\
\hline 2.3 & 7.0 \\
\hline $\begin{array}{l}0.8 \\
2.7 \\
0.9 \\
1.3 \\
2.1\end{array}$ & $\begin{array}{r}13.6 \\
0.9 \\
13.9 \\
4.9 \\
0.7\end{array}$ \\
\hline $\begin{array}{l}1.1 \\
0.3 \\
2.3 \\
1.2 \\
1.0\end{array}$ & $\begin{array}{r}1.4 \\
23.6 \\
7.2 \\
8.4 \\
1.2\end{array}$ \\
\hline $\begin{array}{l}1.6 \\
1.2 \\
1.4 \\
1.0 \\
1.4\end{array}$ & $\begin{array}{r}31.4 \\
5.2 \\
21.3 \\
6.5 \\
7.6\end{array}$ \\
\hline $\begin{array}{l}0.9 \\
0.8 \\
2.0 \\
1.5 \\
0.8\end{array}$ & $\begin{array}{r}7.5 \\
9.1 \\
20.0 \\
9.9 \\
3.7\end{array}$ \\
\hline $\begin{array}{l}0.9 \\
1.2 \\
1.1\end{array}$ & $\begin{array}{l}15.1 \\
14.4 \\
16.8\end{array}$ \\
\hline
\end{tabular}

\begin{tabular}{|c|c|}
\hline 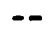 & -- \\
\hline-- & -- \\
\hline-- & - \\
\hline$\cdots$ & - \\
\hline-- & -- \\
\hline$-\overline{-}$ & -- \\
\hline & - \\
\hline-- & -- \\
\hline & -- \\
\hline - & -- \\
\hline-- & - \\
\hline . & $=$ \\
\hline & -- \\
\hline 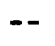 & -- \\
\hline- & - \\
\hline & - \\
\hline & -- \\
\hline & $\cdots$ \\
\hline & -- \\
\hline- & -- \\
\hline & $=-$ \\
\hline & - \\
\hline 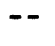 & -- \\
\hline-- & - \\
\hline & -- \\
\hline & $=$ \\
\hline - & - \\
\hline-- & - \\
\hline & -- \\
\hline & -- \\
\hline- & $-\overline{-}$ \\
\hline & \\
\hline & -- \\
\hline & - \\
\hline
\end{tabular}

--
--
--
--
--
--
--
--
--
--
--
--
--
--
--
--
--
--
--
--
--
--
--
--
-
-
-
-
-

\begin{tabular}{|c|c|}
\hline $\begin{array}{l}-- \\
-- \\
-- \\
-- \\
--\end{array}$ & $\begin{array}{l}34.9 \\
62.1 \\
64.3 \\
65.8 \\
68.0\end{array}$ \\
\hline-- & $\begin{array}{l}63.7 \\
66.8 \\
74.6\end{array}$ \\
\hline-- & $\begin{array}{l}47.7 \\
79.8\end{array}$ \\
\hline-- & 61.2 \\
\hline & 67.3 \\
\hline & $\begin{array}{l}48.4 \\
48.3\end{array}$ \\
\hline-- & 66.7 \\
\hline-- & $\begin{array}{l}57.2 \\
87.5 \\
56.3 \\
78.8 \\
86.9\end{array}$ \\
\hline-- & $\begin{array}{l}87.0 \\
31.1 \\
71.7 \\
67.9 \\
87.6\end{array}$ \\
\hline-- & $\begin{array}{l}12.7 \\
76.5 \\
38.3 \\
73.9 \\
65.3\end{array}$ \\
\hline- & $\begin{array}{l}65.3 \\
61.9 \\
28.6 \\
55.8 \\
73.8\end{array}$ \\
\hline $\begin{array}{l}-- \\
--\end{array}$ & $\begin{array}{l}37.8 \\
46.3 \\
37.9\end{array}$ \\
\hline
\end{tabular}

80.57

1, 088.02

$1,094.74$

88.47

89.87

$1,109.02$

$1,122.52$

$1,127.42$

91.27

$1,140.16$

92.67

$1,150.24$

93.27

93.77

$1,159.00$

$1,163.25$

96.07

$1,179.35$

96. 87

99.57

100.47

101.77

103.87

104.97

105. 27

107.57

108. 77

109. 77

111.37

112.57

113.97

114.97

116.37

117.27

118.07

120.07

121.57

122. 37

$1,190.23$

$1,192.66$

$1,205.17$

$1,211.54$

1.213 .01

$1,214.55$

$1,221.63$

$1,238.19$

$1,248.27$

$1,249.47$

$1,299.71$

$1,305.95$

1. 335.77

$1,342.27$

$1,352.91$

$1,359.66$

$1,366.94$

$1,406.94$

$1,421.79$

$1,424.75$

123.27

124.47

$1,438.34$

$1,455.62$

$1,474.10$ 


\begin{tabular}{|c|c|c|c|c|c|c|c|c|c|c|c|}
\hline \multirow[b]{2}{*}{$\begin{array}{c}\text { Bed } \\
\text { no. }\end{array}$} & \multirow[b]{2}{*}{ Rock description } & \multirow[b]{2}{*}{$\begin{array}{l}\text { Sample } \\
\text { no. }\end{array}$} & \multirow{2}{*}{$\underset{\text { (feet) }}{\text { Thickns }}$} & \multicolumn{6}{|c|}{ Chemical analyses (percent) } & \multirow{2}{*}{$\begin{array}{l}\text { Cumulative } \\
\text { thickness } \\
\text { (feet) }\end{array}$} & \multirow{2}{*}{$\begin{array}{l}\text { Thickness } \mathbf{x} \\
\text { percent } \mathrm{P}_{2} \mathrm{O}_{5} \\
\text { (cumulative) }^{5}\end{array}$} \\
\hline & & & & $\mathrm{P}_{2} \mathrm{O}_{5}$ & $\mathrm{Al}_{2} \mathrm{O}_{3}$ & $\mathrm{Fe}_{2} \mathrm{O}_{3}$ & $F$ & $\begin{array}{l}\text { Loss on } \\
\text { ignition }\end{array}$ & $\begin{array}{c}\text { Acid } \\
\text { insoluble }\end{array}$ & & \\
\hline $\begin{array}{l}P-57 \\
P=56 \\
P-55 \\
P-54\end{array}$ & $\begin{array}{l}\text { Mudstone } \\
\text { Mudstone, phosphatic } \\
\text { Mudstone } \\
\text { Mudstone and chert; fos. col. }\end{array}$ & $\begin{array}{l}\text { VEM-561-47 } \\
\text { VEM-560-47 } \\
\text { RAW- } 87-47\end{array}$ & $\begin{array}{l}1.5 \\
1.2 \\
0.6\end{array}$ & $\begin{array}{l}2.8 \\
8.5 \\
1.5\end{array}$ & $\begin{array}{l}-- \\
-- \\
--\end{array}$ & $\begin{array}{l}-- \\
-- \\
--\end{array}$ & $\begin{array}{l}-- \\
-- \\
--\end{array}$ & $\begin{array}{l}-- \\
--\end{array}$ & $\begin{array}{l}70.5 \\
60.3 \\
81.7\end{array}$ & $\begin{array}{l}127.07 \\
128.27 \\
128.87\end{array}$ & $\begin{array}{l}1,478.30 \\
1,488.50 \\
1,489.40\end{array}$ \\
\hline P- 53 & $\begin{array}{l}\text { no. } 47-\mathrm{HW}-266 \\
\text { Muds tone; fos. col. no. } 47-\mathrm{HW}-265\end{array}$ & $\begin{array}{l}\text { RAW- } 86-47 \\
\text { RAW- 85-47 }\end{array}$ & $\begin{array}{l}0.8 \\
1.7\end{array}$ & $\begin{array}{l}0.9 \\
3.2\end{array}$ & 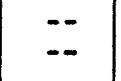 & -- & -- & $\ddot{--}$ & $\begin{array}{l}91.7 \\
80.7\end{array}$ & $\begin{array}{l}129.67 \\
131.37\end{array}$ & $\begin{array}{l}1,490.12 \\
1,495.56\end{array}$ \\
\hline $\begin{array}{l}P-52 \\
P-51 \\
P-50 \\
P-49 \\
P-48\end{array}$ & $\begin{array}{l}\text { Mudstone } \\
\text { Mudstone, phosphatic } \\
\text { Mudstone; fos. col. no. } 47-\mathrm{HW}-264 \\
\text { Mudstone } \\
\text { Mudstone, phosphatic }\end{array}$ & $\begin{array}{l}\text { RAW- } 84-47 \\
\text { RAW- } 83-47 \\
\text { RAW- } 82-47 \\
\text { RAW- 81-47 } \\
\text { RAW- } 80-47\end{array}$ & $\begin{array}{l}0.9 \\
0.9 \\
2.1 \\
1.6 \\
1.9\end{array}$ & $\begin{array}{r}1.5 \\
8.8 \\
5.2 \\
0.7 \\
11.6\end{array}$ & $\begin{array}{l}-- \\
-- \\
-- \\
--\end{array}$ & $\begin{array}{l}-- \\
-- \\
-- \\
-\end{array}$ & $\begin{array}{l}-- \\
-- \\
-- \\
--\end{array}$ & $\begin{array}{l}-- \\
-- \\
-- \\
--\end{array}$ & $\begin{array}{l}84.2 \\
64.2 \\
75.2 \\
89.6 \\
57.1\end{array}$ & $\begin{array}{l}132.27 \\
133.17 \\
135.27 \\
136.87 \\
138.77\end{array}$ & $\begin{array}{l}1,496.91 \\
1,504.83 \\
1,515.75 \\
1,516.87 \\
1,538.91\end{array}$ \\
\hline $\begin{array}{l}P-47 \\
P-46 \\
P-45\end{array}$ & $\begin{array}{l}\text { Mudstone; fos. col. no. } 47-\mathrm{HW}-263 \\
\text { Mudstone }\end{array}$ & $\begin{array}{l}\text { RAW- } 79-47 \\
\text { RAW- 78-47 }\end{array}$ & $\begin{array}{l}1.2 \\
0.7\end{array}$ & $\begin{array}{l}7.8 \\
3.4\end{array}$ & $=$ & $=$ & $=$ & $=-$ & $\begin{array}{l}68.8 \\
82.0\end{array}$ & $\begin{array}{l}139.97 \\
140.67\end{array}$ & $\begin{array}{l}1,548.27 \\
1,550.65\end{array}$ \\
\hline $\begin{array}{l}\text { P- } 44 \\
P-43\end{array}$ & $\begin{array}{l}\text { col. no. 47-HW-262 } \\
\text { Mudstone, phosphatic } \\
\text { Mudstone, phosphatic }\end{array}$ & $\begin{array}{l}\text { RAW- } 77-47 \\
\text { RAW- } 76-47 \\
\text { RAW- } 75-47\end{array}$ & $\begin{array}{l}1.1 \\
1.7 \\
0.5\end{array}$ & $\begin{array}{r}9.4 \\
16.4 \\
13.0\end{array}$ & $\begin{array}{l}-- \\
--\end{array}$ & $\begin{array}{l}-- \\
--\end{array}$ & $\begin{array}{l}-- \\
-- \\
--\end{array}$ & $\begin{array}{l}-- \\
--\end{array}$ & $\begin{array}{l}64.2 \\
42.7 \\
50.8\end{array}$ & $\begin{array}{l}141.77 \\
143.47 \\
143.97\end{array}$ & $\begin{array}{l}1,560.99 \\
1,588.87 \\
1,595.37\end{array}$ \\
\hline $\begin{array}{l}P-42 \\
P-41 \\
P-40 \\
P-39 \\
P-38\end{array}$ & $\begin{array}{l}\text { Mudstone } \\
\text { Mudstone, phosphatic } \\
\text { Mudstone, phosphatic } \\
\text { Mudstone, phosphatic } \\
\text { Phosphate rock, argillaceous }\end{array}$ & $\begin{array}{l}\text { RAW- } 74-47 \\
\text { VEM-559-47 } \\
\text { VEM } 558-47 \\
\text { VEM-557-47 } \\
\text { VEM-556-47 }\end{array}$ & $\begin{array}{l}0.6 \\
1.8 \\
2.2 \\
2.6 \\
0.9\end{array}$ & $\begin{array}{l}2.8 \\
16.0 \\
10.2 \\
11.5 \\
19.7\end{array}$ & $\begin{array}{l}-- \\
\because- \\
=- \\
=-\end{array}$ & $\begin{array}{l}-- \\
-- \\
-- \\
--\end{array}$ & $\begin{array}{l}-- \\
-- \\
-- \\
--\end{array}$ & $\begin{array}{l}-- \\
-- \\
-- \\
--\end{array}$ & $\begin{array}{l}80.0 \\
40.9 \\
57.4 \\
53.0 \\
35.8\end{array}$ & $\begin{array}{l}144.57 \\
146.37 \\
148.57 \\
151.17 \\
152.07\end{array}$ & $\begin{array}{l}1,597.05 \\
1,625.85 \\
1,648.29 \\
1,678.19 \\
1,695.92\end{array}$ \\
\hline $\begin{array}{l}P-37 \\
P-36 \\
P-35 \\
P-34 \\
P-33\end{array}$ & $\begin{array}{l}\text { Phosphate rock, argillaceous } \\
\text { Phosphate rock and mudstone } \\
\text { Phosphate rock } \\
\text { Phosphate rock and mudstone } \\
\text { Phosphate rock and mudstone }\end{array}$ & $\begin{array}{l}\text { VEM-555-47 } \\
\text { VEM } 554-47 \\
\text { VEM }-553-47 \\
\text { VEM-552-47 } \\
\text { VEM }-551-47\end{array}$ & $\begin{array}{l}0.8 \\
1.0 \\
1.8 \\
1.3 \\
1.7\end{array}$ & $\begin{array}{l}18.2 \\
22.9 \\
26.3 \\
31.5 \\
21.7\end{array}$ & $\begin{array}{l}-- \\
-- \\
-- \\
-\end{array}$ & $\begin{array}{l}-- \\
-- \\
--\end{array}$ & $\begin{array}{l}-- \\
-- \\
--\end{array}$ & $\begin{array}{l}-- \\
-- \\
-- \\
--\end{array}$ & $\begin{array}{l}38.9 \\
24.9 \\
18.2 \\
11.0 \\
29.3\end{array}$ & $\begin{array}{l}152.87 \\
153.87 \\
155.67 \\
156.97 \\
158.67\end{array}$ & $\begin{array}{l}1,710.48 \\
1,733.38 \\
1,780.72 \\
1,821.67 \\
1,858.56\end{array}$ \\
\hline $\begin{array}{l}P-32 \\
P-31\end{array}$ & $\begin{array}{l}\text { Mudstone, phosphatic } \\
\text { Mudstone, phosphatic; fos. col. }\end{array}$ & VEM $-550-47$ & 1.3 & 11.0 & -- & -- & - & -- & 52.0 & 159.97 & $1,872.86$ \\
\hline $\begin{array}{l}P-30 \\
P-29 \\
P-28\end{array}$ & $\begin{array}{l}\text { no. } 47-\mathrm{HW}-261 \\
\text { Mudstone, phosphatic } \\
\text { Mudstone, phosphatic } \\
\text { Mudstone, phosphatic }\end{array}$ & $\begin{array}{l}\text { VEM-549-47 } \\
\text { VEM-548-47 } \\
\text { VEM-547-47 } \\
\text { VEM-546-47 }\end{array}$ & $\begin{array}{l}1.6 \\
1.4 \\
1.7 \\
1.5\end{array}$ & $\begin{array}{l}12.0 \\
13.8 \\
12.8 \\
11.9\end{array}$ & $\begin{array}{l}-- \\
-- \\
--\end{array}$ & $\begin{array}{l}-- \\
-- \\
--\end{array}$ & $\begin{array}{l}-- \\
-- \\
--\end{array}$ & $\begin{array}{l}-- \\
-- \\
--\end{array}$ & $\begin{array}{l}53.3 \\
50.4 \\
52.8 \\
52.5\end{array}$ & $\begin{array}{l}161.57 \\
162.97 \\
164.67 \\
166.17\end{array}$ & $\begin{array}{l}1,892.06 \\
1,911.38 \\
1,933.14 \\
1,950.99\end{array}$ \\
\hline $\begin{array}{l}P-27 \\
P-26 \\
P-25 \\
P-24 \\
P-23\end{array}$ & $\begin{array}{l}\text { Mudstone, phosphatic; fos. col. } \\
\text { no. } 47-\mathrm{HW}-260 \\
\text { Limestone, argillaceous } \\
\text { Mudstone, calcareous } \\
\text { Phosphate rock, argillaceous } \\
\text { Phosphate rock, argillaceous }\end{array}$ & $\begin{array}{l}\text { VEM-545-47 } \\
\text { VEM-544-47 } \\
\text { VEM-543-47 } \\
\text { VEM-542-47 } \\
\text { VEM-541-47 }\end{array}$ & $\begin{array}{l}0.9 \\
1.5 \\
1.0 \\
2.2 \\
1.7\end{array}$ & $\begin{array}{r}12.6 \\
1.2 \\
4.6 \\
24.1 \\
25.5\end{array}$ & $\begin{array}{l}-- \\
-- \\
3.1 \\
2.4\end{array}$ & $\begin{array}{l}-- \\
-- \\
-- \\
1.25 \\
1.08\end{array}$ & $\begin{array}{l}-- \\
-- \\
0.39 \\
-- \\
2.35\end{array}$ & $\begin{array}{l}-- \\
-- \\
8.12 \\
9.14\end{array}$ & $\begin{array}{l}52.2 \\
26.5 \\
44.5 \\
24.8 \\
20.8\end{array}$ & $\begin{array}{l}167.07 \\
168.57 \\
169.57 \\
171.77 \\
173.47\end{array}$ & $\begin{array}{l}1,962.33 \\
1,964.13 \\
1,968.73 \\
2,021.75 \\
2,065.10\end{array}$ \\
\hline $\begin{array}{l}P-22 \\
P-.21\end{array}$ & $\begin{array}{l}\text { Phosphate rock, argillaceous } \\
\text { Mudstone, phosphatic }\end{array}$ & $\begin{array}{l}\text { VEM-540-47 } \\
\text { VEM-539-47 }\end{array}$ & $\begin{array}{l}0.6 \\
0.4\end{array}$ & $\begin{array}{l}20.4 \\
15.0\end{array}$ & $\begin{array}{l}5.5 \\
6.2\end{array}$ & $\begin{array}{l}1.81 \\
2.09\end{array}$ & -- & $\begin{array}{l}7.22 \\
7.28\end{array}$ & $\begin{array}{l}34.9 \\
48.7\end{array}$ & $\begin{array}{l}174.07 \\
174.47\end{array}$ & $\begin{array}{l}2,077.34 \\
2,083.34\end{array}$ \\
\hline
\end{tabular}




\begin{tabular}{|c|c|c|c|c|c|c|c|c|c|c|c|}
\hline $\begin{array}{l}P-20 \\
P-19 \\
P-18 \\
P-17 \\
P=16\end{array}$ & $\begin{array}{l}\text { Mudstone, phosphatic } \\
\text { Mudstone } \\
\text { Phosphate rock, argillaceous; } \\
\text { fos. col. no. } 47-\mathrm{HW}-247 \\
\text { Phosphate rock } \\
\text { Phosphate rock }\end{array}$ & $\begin{array}{l}\text { VEM-538-47 } \\
\text { VEM-537-47 } \\
\text { VEM-536-47 } \\
\text { VEM-535-47 } \\
\text { VEM-534-47 }\end{array}$ & $\begin{array}{l}1.2 \\
0.4 \\
1.8 \\
1.7 \\
1.9\end{array}$ & $\begin{array}{r}14.1 \\
6.3 \\
27.1 \\
28.6 \\
29.1\end{array}$ & $\begin{array}{l}6.9 \\
9.7 \\
2.5 \\
2.9\end{array}$ & $\begin{array}{l}2.30 \\
3.17 \\
-- \\
1.02 \\
1.07\end{array}$ & $\begin{array}{c}1.52 \\
0.62 \\
-- \\
-- \\
--\end{array}$ & $\begin{array}{l}6.96 \\
5.36 \\
6.48 \\
6.12 \\
6.54\end{array}$ & $\begin{array}{l}49.6 \\
70.7 \\
21.7 \\
18.8 \\
19.2\end{array}$ & $\begin{array}{l}175.67 \\
176.07 \\
177.87 \\
179.57 \\
181.47\end{array}$ & $\begin{array}{l}2,100.26 \\
2,102.78 \\
2,151.56 \\
2,200.18 \\
2,255.47\end{array}$ \\
\hline $\begin{array}{l}P-15 \\
P-14 \\
P-13 \\
P-12 \\
P-11\end{array}$ & $\begin{array}{l}\text { Mudstone, phosphatic } \\
\text { Phosphate rock, argillaceous } \\
\text { Mudstone, phosphatic } \\
\text { Phosphate rock, argillaceous; } \\
\text { fos, col. no. } 47 \text {-HW-246 } \\
\text { Phosphate rock, argillaceous }\end{array}$ & $\begin{array}{l}\text { VEM-533-47 } \\
\text { VEM-532-47 } \\
\text { VEM }-531-47 \\
\text { VEM-530-47 } \\
\text { VEM-529-47 }\end{array}$ & $\begin{array}{l}0.5 \\
0.7 \\
0.5 \\
2.4 \\
0.8\end{array}$ & $\begin{array}{l}12.8 \\
26.9 \\
15.9 \\
24.5 \\
21.4\end{array}$ & $\begin{array}{l}8.0 \\
4.1 \\
6.9 \\
3.9 \\
5.9\end{array}$ & $\begin{array}{l}2.40 \\
1.25 \\
2.31 \\
1.21 \\
2.46\end{array}$ & $\begin{array}{l}-- \\
1.59 \\
-- \\
--\end{array}$ & $\begin{array}{l}6.64 \\
5.70 \\
7.44 \\
6.50 \\
5.34\end{array}$ & $\begin{array}{l}54.0 \\
22.2 \\
43.9 \\
26.2 \\
33.9\end{array}$ & $\begin{array}{l}181.97 \\
182.67 \\
183.17 \\
185.57 \\
186.37\end{array}$ & $\begin{array}{l}2,261.87 \\
2,280.70 \\
2,288.65 \\
2,347.45 \\
2,364.57\end{array}$ \\
\hline $\begin{array}{l}P-10 \\
P-\quad 9 \\
P-\quad 8 \\
P-\quad 7 \\
P-\quad 6\end{array}$ & $\begin{array}{l}\text { Phosphate rock } \\
\text { Phosphate rock } \\
\text { Phosphate rock } \\
\text { Phosphate rock } \\
\text { Phosphate rock }\end{array}$ & $\begin{array}{l}\text { VEM-528-47 } \\
\text { VEM-527-47 } \\
\text { VEM-526-47 } \\
\text { VEM-525-47 } \\
\text { VEM-524-47 }\end{array}$ & $\begin{array}{l}1.6 \\
1.2 \\
1.3 \\
1.5 \\
0.7\end{array}$ & $\begin{array}{l}33.2 \\
32.7 \\
33.7 \\
33.4 \\
32.3\end{array}$ & $\begin{array}{l}1.0 \\
1.4 \\
1.2 \\
0.53 \\
1.7\end{array}$ & $\begin{array}{l}0.54 \\
0.66 \\
0.56 \\
0.44 \\
0.82\end{array}$ & $\begin{array}{c}2.84 \\
-- \\
-- \\
-- \\
--\end{array}$ & $\begin{array}{l}6.02 \\
5.98 \\
5.64 \\
7.08 \\
5.86\end{array}$ & $\begin{array}{l}6.4 \\
6.0 \\
4.4 \\
4.9 \\
8.4\end{array}$ & $\begin{array}{l}187.97 \\
189.17 \\
190.47 \\
191.97 \\
192.67\end{array}$ & $\begin{array}{l}2,417.69 \\
2,456.93 \\
2,500.74 \\
2,550.84 \\
2,573.45\end{array}$ \\
\hline $\begin{array}{ll}P- & 5 \\
P- & 4 \\
P- & 3 \\
P- & 2 \\
P- & 1\end{array}$ & $\begin{array}{l}\text { Phosphate rock, argillaceous } \\
\text { Mudstone } \\
\text { Mudstone } \\
\text { Phosphate rock } \\
\text { Phosphate rock and mudstone }\end{array}$ & $\begin{array}{l}\text { VEM-523-47 } \\
\text { VEM-522-47 } \\
\text { VEM-521-47 } \\
\text { VEM-520-47 } \\
\text { VEM-519-47 }\end{array}$ & $\begin{array}{l}1.0 \\
1.0 \\
2.5 \\
0.6 \\
0.6\end{array}$ & $\begin{array}{r}25.4 \\
3.9 \\
1.2 \\
33.7 \\
26.2\end{array}$ & $\begin{array}{r}4.5 \\
9.7 \\
10 . \\
1.4 \\
5.1\end{array}$ & $\begin{array}{l}1.27 \\
4.18 \\
3.61 \\
0.66 \\
2.32\end{array}$ & $\begin{array}{l}-- \\
-- \\
-- \\
3 .-\end{array}$ & $\begin{array}{l}6.46 \\
7.00 \\
5.32 \\
4.06 \\
5.02\end{array}$ & $\begin{array}{r}22.9 \\
69.7 \\
78.7 \\
7.2 \\
19.0\end{array}$ & $\begin{array}{l}193.67 \\
194.67 \\
197.17 \\
197.77 \\
198.37\end{array}$ & $\begin{array}{l}2,598.85 \\
2,602.75 \\
2,605.75 \\
2,625.97 \\
2,641.69\end{array}$ \\
\hline \multicolumn{12}{|c|}{ Wells formation } \\
\hline $\begin{array}{l}C w-1 \\
C w-2 \\
C w-3\end{array}$ & $\begin{array}{l}\text { Dolomite; fos. col. no. } \\
47-\mathrm{HW}-244 \\
\text { Phosphate rock } \\
\text { Dolomite; fos. col. no. } \\
\text { 47-HW-245 }\end{array}$ & $\begin{array}{c}\text { VEM-518-47 } \\
-- \\
--\end{array}$ & $\begin{array}{l}3.2 \\
0.1 \\
4.0\end{array}$ & $\begin{array}{l}1.0 \\
-- \\
--\end{array}$ & -- & -- & -- & -- & $\begin{array}{l}4.8 \\
-- \\
\ldots\end{array}$ & $\begin{array}{l}3.2 \\
3.3 \\
7.3\end{array}$ & $\begin{array}{l}3.20 \\
--\end{array}$ \\
\hline
\end{tabular}


WEST DAIRY, IDAHO. LOT NO. 1208.

Phosphatic shale member and part of Rex member of Phosphoria formation sampled in bulldozer trench near SE corner of NE $\frac{1}{4}$ SE $\frac{1}{4}$ sec. 19. T. 9 S., R. 44 E., Caribou County, Idaho, on west limb of Dairy syncline. Beds strike N. $64^{\circ}$ W. and dip $85^{\bullet}$ NE. Section measured by F. C. Armstrong, V. E. McKelvey, L. E. Smith, and R. A. Weeks and sampled by O. A. Payne and R. A. Smart in September 1947. Samples analyzed for $\mathrm{P}_{2} \mathrm{O}_{5}$ and acid insoluble by U. S. Bureau of Mines Laboratory, Albany, Oregon, and for other constituents by Trace Elements Section Laboratory, U. S. Geological Survey, Washington, D. C.

\begin{tabular}{|c|c|c|c|c|c|c|c|c|c|c|}
\hline \multirow{2}{*}{$\begin{array}{c}\text { Bed } \\
\text { no. }\end{array}$} & \multirow[b]{2}{*}{ Rock description } & \multirow{2}{*}{$\begin{array}{c}\text { Sample } \\
\text { no. }\end{array}$} & \multirow{2}{*}{$\begin{array}{c}\text { Thickness } \\
\text { (feet) }\end{array}$} & \multicolumn{5}{|c|}{ Chemical analyses (percent) } & \multirow{2}{*}{$\begin{array}{c}\text { Cumulative } \\
\text { thickness } \\
\text { (feet) }\end{array}$} & \multirow{2}{*}{$\begin{array}{l}\text { Thickness } x \\
\text { percent } \mathrm{P}_{2} \mathrm{O}_{5} \\
\text { (cumulative) }\end{array}$} \\
\hline & & & & $\mathrm{P}_{2} \mathrm{O}_{5}$ & $\mathrm{Al}_{2} \mathrm{O}_{3}$ & $\mathrm{Fe}_{2} \mathrm{O}_{3}$ & $\begin{array}{l}\text { Loss on } \\
\text { ignition }\end{array}$ & $\begin{array}{c}\text { Acid } \\
\text { insoluble }\end{array}$ & & \\
\hline
\end{tabular}

Rex member of Phosphoria formation - basal beds only

\begin{tabular}{ll|l}
\hline$R-$ & 4 & Mudstone and chert; fos. col. no. \\
& & $47-H W-1391$ \\
$R-$ & 3 & Chert \\
$R-$ & 2 & Mudstone and chert \\
$R-$ & 1 & Chert, phosphatic \\
\hline
\end{tabular}

\begin{tabular}{l|l} 
& \\
FCA-267-47 & 1.7 \\
FCA-268-47 & 1.0 \\
FCA-269-47 & 0.2 \\
FCA-270-47 & 0.2
\end{tabular}

\begin{tabular}{r|r}
1.6 & \\
0.5 & \\
1.7 & \\
15.6 &
\end{tabular}

\begin{tabular}{l|l|l}
-- & -- & - \\
-- & -- & - \\
-- & -- & - \\
-- & -- & -
\end{tabular}

Phosphatic shale member of Phosphoria formation

\begin{tabular}{|c|c|}
\hline $\begin{array}{l}P-193 \\
P-192 \\
P-191 \\
P-190 \\
P-189\end{array}$ & $\begin{array}{l}\text { Mudstone, phosphatic } \\
\text { Mudstone } \\
\text { Mudstone, phosphatic } \\
\text { Mudstone } \\
\text { Mudstone; fos. col. no. 47-HW-140 }\end{array}$ \\
\hline $\begin{array}{l}P-188 \\
P-187 \\
P-186 \\
P-185 \\
P=184\end{array}$ & $\begin{array}{l}\text { Mudstone; fos. col. no. } 47-\mathrm{HW}-141 \\
\text { Mudstone; fos. col. no. } 47-\mathrm{HW}-142 \\
\text { Mudstone } \\
\text { Mudstone, phosphatic } \\
\text { Mudstone }\end{array}$ \\
\hline $\begin{array}{l}P-183 \\
P-182 \\
P-181 \\
P-180 \\
P-179\end{array}$ & $\begin{array}{l}\text { Phosphate rock, argillaceous } \\
\text { Phosphate rock } \\
\text { Mudstone } \\
\text { Mudstone; fos. col. no. } 47-\mathrm{HW}-143 \\
\text { Mudstone }\end{array}$ \\
\hline $\begin{array}{l}P-178 \\
P-177 \\
P-176 \\
P-175 \\
P-174\end{array}$ & $\begin{array}{l}\text { Mudstone; fos. col. no. } 47-\mathrm{HW}-144 \\
\text { Phosphate rock } \\
\text { Mudstone } \\
\text { Mudstone; fos. col. no. } 47-\mathrm{HW}-145 \\
\text { Phosphate rock }\end{array}$ \\
\hline $\begin{array}{l}P-173 \\
P-172 \\
P-171 \\
P-170 \\
P-169\end{array}$ & $\begin{array}{l}\text { Phosphate rock } \\
\text { Phosphate rock } \\
\text { Phosphate rock } \\
\text { Mudstone, phosphatic } \\
\text { Phosphate rock, argillaceous }\end{array}$ \\
\hline
\end{tabular}

FCA-271-47
FCA-272-47
FCA-273-47
FCA-281-47
FCA-280-47
FCA-279-47
FCA-278=47
FCA- 277-47
FCA-276-47
FCA- 275-47
FCA-274-47
RAW- $1-47$
RAW- $2-47$
RAW- $3-47$
RAW- $4-47$
RAW- $5-47$
RAW- $6-47$
RAW- $7-47$
RAW- $8-47$
RAW- $9-47$
RAW- $10-47$
RAW- $11-47$
RAW- $12-47$
RAW- $13-47$
RAW- $14-47$

\begin{tabular}{l|r|r|l|}
0.2 & 8.7 & -- & -- \\
0.8 & 0.8 & -- & -- \\
0.4 & 7.8 & -- & -- \\
1.4 & 3.6 & -- & -- \\
2.9 & 1.3 & -- & -- \\
1.7 & 1.8 & - & \\
0.5 & 3.7 & -- & -- \\
0.9 & 3.2 & -- & -- \\
0.35 & 15.7 & -- & -- \\
1.4 & 1.7 & -- & -- \\
0.35 & 19.0 & -- & -- \\
0.2 & 32.5 & -- & -- \\
1.1 & 2.8 & -- & - \\
0.9 & 0.6 & -- & -- \\
1.5 & 4.7 & -- & -- \\
1.3 & 2.9 & -- & - \\
0.5 & 30.5 & -- & -- \\
1.3 & 3.7 & -- & -- \\
1.6 & 1.0 & -- & -- \\
0.5 & 32.3 & 1.8 & 0.40 \\
0.9 & 32.8 & 1.7 & 0.45 \\
0.5 & 33.3 & 2.2 & 0.33 \\
0.9 & 33.6 & 2.0 & 0.35 \\
0.7 & 14.5 & 7.8 & 2.50 \\
0.8 & 18.2 & 5.5 & 1.83 \\
& & & \\
& & &
\end{tabular}

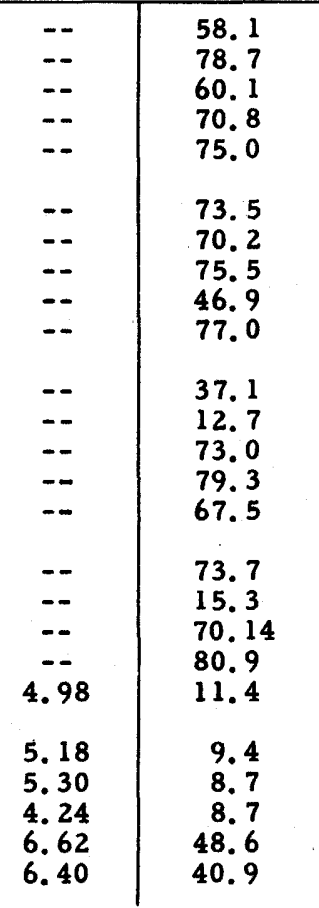

\begin{tabular}{l|r}
0.2 & 1.74 \\
1.0 & 2.38 \\
1.4 & 5.50 \\
2.8 & 10.54 \\
5.7 & 14.31 \\
& \\
7.4 & 17.37 \\
7.9 & 19.22 \\
8.8 & 22.10 \\
9.15 & 27.60 \\
10.55 & 29.98 \\
10.90 & 36.63 \\
11.10 & 43.13 \\
12.20 & 46.21 \\
13.10 & 46.75 \\
14.60 & 53.80 \\
15.90 & \\
16.40 & 57.57 \\
17.70 & 72.82 \\
19.30 & 77.63 \\
19.80 & 79.23 \\
20.70 & 95.38 \\
21.20 & 124.90 \\
22.10 & 141.55 \\
22.80 & 171.79 \\
23.60 & 181.94 \\
& 196.50
\end{tabular}




\begin{tabular}{|c|c|}
\hline $\begin{array}{l}P-168 \\
P-167 \\
P-166\end{array}$ & $\begin{array}{l}\text { Phosphate rock, argillaceous } \\
\text { Phosphate rock } \\
\text { Phosphate rock and phosphatic } \\
\text { mudstone }\end{array}$ \\
\hline $\begin{array}{l}P-165 \\
P-164\end{array}$ & $\begin{array}{l}\text { Phosphate rock } \\
\text { Phosphate rock and argillaceous } \\
\text { phosphate rock }\end{array}$ \\
\hline $\begin{array}{l}P-163 \\
P-162 \\
P-161 \\
P-160 \\
P-159\end{array}$ & $\begin{array}{l}\text { Phosphate rock, argillaceous } \\
\text { Phosphate rock, argillaceous } \\
\text { Phosphate rock, argillaceous } \\
\text { Mudstone, phosphatic } \\
\text { Phosphate rock and phosphatic } \\
\text { mudstone }\end{array}$ \\
\hline$P-158$ & $\begin{array}{l}\text { Phosphate rock, argillaceous and } \\
\text { phosphate rock }\end{array}$ \\
\hline $\begin{array}{l}P-157 \\
P-156 \\
P-155 \\
P-154\end{array}$ & $\begin{array}{l}\text { Phosphate rock } \\
\text { Phosphate rock } \\
\text { Phosphate rock } \\
\text { Phosphate rock }\end{array}$ \\
\hline $\begin{array}{l}P-153 \\
P-152 \\
P-151 \\
P-150\end{array}$ & $\begin{array}{l}\text { Phosphate rock, argillaceous } \\
\text { Phosphate rock, argillaceous } \\
\text { Phosphate rock, argillaceous } \\
\text { Phosphate rock, argillaceous; fos. } \\
\text { col. no. 47-HW-146 }\end{array}$ \\
\hline$P-149$ & Phosphate rock, argillaceous \\
\hline $\begin{array}{l}P=148 \\
P-147 \\
P=146 \\
P=145 \\
P-144\end{array}$ & $\begin{array}{l}\text { Mudstone, phosphatic } \\
\text { Mudstone, phosphatic } \\
\text { Mudstone } \\
\text { Mudstone } \\
\text { Mudstone, phosphatic; fos. col. no. } \\
\quad 47-\text { HW-175 }\end{array}$ \\
\hline $\begin{array}{l}P-143 \\
P-142 \\
P-141 \\
P-140\end{array}$ & $\begin{array}{l}\text { Mudstone, phosphatic } \\
\text { Mudstone } \\
\text { Mudstone, phosphatic } \\
\text { Mudstone, phosphatic; fos. col. no. } \\
47-H W-147\end{array}$ \\
\hline$P-139$ & Mudstone \\
\hline $\begin{array}{l}P-138 \\
P=137 \\
P=136 \\
P=135 \\
P-134\end{array}$ & $\begin{array}{l}\text { Mudstone, phosphatic } \\
\text { Mudstone } \\
\text { Mudstone, phosphatic } \\
\text { Mudstone, phosphatic } \\
\text { Mudstone }\end{array}$ \\
\hline 1 & $\mathbf{M u}$ \\
\hline
\end{tabular}

\begin{tabular}{|c|c|c|c|c|c|c|c|c|}
\hline $\begin{array}{l}\text { RAW- } 15-47 \\
\text { RAW- } 16-47\end{array}$ & $\begin{array}{l}0.9 \\
0.7\end{array}$ & $\begin{array}{l}20.2 \\
33.9\end{array}$ & $\begin{array}{l}5.0 \\
1.2\end{array}$ & $\begin{array}{l}1.65 \\
0.63\end{array}$ & $\begin{array}{l}5.80 \\
4.58\end{array}$ & $\begin{array}{r}36.6 \\
5.8\end{array}$ & $\begin{array}{l}24.50 \\
25.20\end{array}$ & $\begin{array}{l}214.68 \\
238.41\end{array}$ \\
\hline $\begin{array}{l}\text { RAW- } 17-47 \\
\text { VEM-304-47 }\end{array}$ & $\begin{array}{l}1.0 \\
0.3\end{array}$ & $\begin{array}{l}16.9 \\
33.8\end{array}$ & $\begin{array}{l}7.3 \\
1.5\end{array}$ & $\begin{array}{l}2.00 \\
0.70\end{array}$ & $\begin{array}{l}5.88 \\
4.24\end{array}$ & $\begin{array}{r}44.5 \\
9.0\end{array}$ & $\begin{array}{l}26.20 \\
26.50\end{array}$ & $\begin{array}{l}255.31 \\
265.45\end{array}$ \\
\hline VEM-303-47 & 1.0 & 25.9 & 3.2 & 1.15 & 5.20 & 25.6 & 27.50 & 291.35 \\
\hline $\begin{array}{l}\text { VEM-302-47 } \\
\text { VEM-301-47 } \\
\text { VEM-300-47 } \\
\text { VEM-299-4 }\end{array}$ & $\begin{array}{l}0.8 \\
1.3 \\
0.3 \\
0.9\end{array}$ & $\begin{array}{l}26.1 \\
20.5 \\
20.9 \\
11.4\end{array}$ & $\begin{array}{l}3.9 \\
5.2 \\
6.6 \\
9.5\end{array}$ & $\begin{array}{l}1.26 \\
1.75 \\
1.85 \\
2.91\end{array}$ & $\begin{array}{l}6.86 \\
6.20 \\
7.60 \\
8.24\end{array}$ & $\begin{array}{l}21.0 \\
37.0 \\
32.3 \\
53.3\end{array}$ & $\begin{array}{l}28.30 \\
29.60 \\
29.90 \\
30.80\end{array}$ & $\begin{array}{l}312.23 \\
338.88 \\
345.15 \\
355.41\end{array}$ \\
\hline VEM-298-47 & 0.7 & 12.6 & 8.07 & 2.73 & 8.32 & 50.3 & 31.50 & 364.23 \\
\hline $\begin{array}{l}\text { VEM-297-47 } \\
\text { VEM-296-47 } \\
\text { VEM-295-47 } \\
\text { VEM-294-47 } \\
\text { VEM-293-47 }\end{array}$ & $\begin{array}{l}0.7 \\
0.4 \\
0.6 \\
1.0 \\
0.8\end{array}$ & $\begin{array}{l}23.0 \\
32.8 \\
33.1 \\
32.3 \\
28.1\end{array}$ & $\begin{array}{l}5.3 \\
1.3 \\
1.5 \\
1.9 \\
2.4\end{array}$ & $\begin{array}{l}1.63 \\
0.59 \\
0.52 \\
0.68 \\
0.43\end{array}$ & $\begin{array}{l}5.88 \\
4.98 \\
4.70 \\
5.88 \\
7.22\end{array}$ & $\begin{array}{l}29.5 \\
10.6 \\
10.0 \\
10.4 \\
17.6\end{array}$ & $\begin{array}{l}32.20 \\
32.60 \\
33.20 \\
34.20 \\
35.00\end{array}$ & $\begin{array}{l}380.33 \\
393.45 \\
413.31 \\
445.61 \\
468.09\end{array}$ \\
\hline $\begin{array}{l}\text { RAG- } 131-47 \\
\text { RAG- } 130-47 \\
\text { RAG- } 129-47\end{array}$ & $\begin{array}{l}1.0 \\
1.1 \\
1.5\end{array}$ & $\begin{array}{l}20.1 \\
21.4 \\
23.1\end{array}$ & $\begin{array}{l}5.7 \\
5.2 \\
4.4\end{array}$ & $\begin{array}{l}1.68 \\
1.68 \\
1.58\end{array}$ & $\begin{array}{l}13.7 \\
13.8 \\
13.1\end{array}$ & $\begin{array}{l}27.4 \\
25.0 \\
21.5\end{array}$ & $\begin{array}{l}36.00 \\
37.10 \\
38.60\end{array}$ & $\begin{array}{l}488.19 \\
511.73 \\
546.38\end{array}$ \\
\hline $\begin{array}{l}\text { RAG- } 128-47 \\
\text { RAG- } 127-47\end{array}$ & $\begin{array}{l}1.0 \\
1.22\end{array}$ & $\begin{array}{l}21.9 \\
19.6\end{array}$ & $\begin{array}{l}5.1 \\
5.7\end{array}$ & $\begin{array}{l}1.70 \\
2.08\end{array}$ & $\begin{array}{l}13.0 \\
11.9\end{array}$ & $\begin{array}{l}26.3 \\
31.4\end{array}$ & $\begin{array}{l}39.60 \\
40.82\end{array}$ & $\begin{array}{l}568.28 \\
592.18\end{array}$ \\
\hline $\begin{array}{l}\text { RAG- } 126-47 \\
\text { RAG- } 125-47 \\
\text { RAG- } 108-47 \\
\text { RAG- } 107-47\end{array}$ & $\begin{array}{l}1.05 \\
1.5 \\
0.95 \\
0.7\end{array}$ & $\begin{array}{r}15.5 \\
12.4 \\
7.0 \\
1.7\end{array}$ & $\begin{array}{l}-- \\
-- \\
-- \\
--\end{array}$ & $\begin{array}{l}-- \\
-- \\
--\end{array}$ & $\begin{array}{l}-- \\
-- \\
--\end{array}$ & $\begin{array}{l}39.2 \\
49.3 \\
68.6 \\
78.7\end{array}$ & $\begin{array}{l}41.87 \\
43.37 \\
44.32 \\
45.02\end{array}$ & $\begin{array}{l}608.48 \\
627.08 \\
633.73 \\
634.92\end{array}$ \\
\hline RAG- $124-47$ & 2.6 & 13.2 & - & - & $\ldots$ & 49.4 & 47.62 & 669.24 \\
\hline $\begin{array}{l}\text { RMC- } 89-47 \\
\text { RMC- } 88-47 \\
\text { RMC- } 87-47\end{array}$ & $\begin{array}{l}1.2 \\
0.8 \\
1.1\end{array}$ & $\begin{array}{r}13.0 \\
5.6 \\
7.9\end{array}$ & $\begin{array}{l}-- \\
--\end{array}$ & $\begin{array}{l}-- \\
--\end{array}$ & $\begin{array}{l}-- \\
-- \\
--\end{array}$ & $\begin{array}{l}53.1 \\
72.0 \\
66.6\end{array}$ & $\begin{array}{l}48.82 \\
49.62 \\
50.72\end{array}$ & $\begin{array}{l}684.84 \\
689.32 \\
698.01\end{array}$ \\
\hline $\begin{array}{l}\text { RMC- } 86-47 \\
\text { RMC- } 85-47\end{array}$ & $\begin{array}{l}0.7 \\
1.0\end{array}$ & $\begin{array}{r}12.3 \\
1.6\end{array}$ & -- & $=$ & -- & $\begin{array}{l}55.7 \\
78.2\end{array}$ & $\begin{array}{l}51.42 \\
52.42\end{array}$ & $\begin{array}{l}706.62 \\
708.22\end{array}$ \\
\hline $\begin{array}{l}\text { RMC- } 84-47 \\
\text { RMC- } 83-47 \\
\text { RMC- } 82-47 \\
\text { VEM- } 310-47 \\
\text { VEM-309-47 }\end{array}$ & $\begin{array}{l}2.4 \\
1.6 \\
0.9 \\
0.7 \\
2.3\end{array}$ & $\begin{array}{r}8.5 \\
3.3 \\
10.9 \\
12.6 \\
7.7\end{array}$ & $\begin{array}{l}-- \\
-- \\
-- \\
--\end{array}$ & $\begin{array}{l}-- \\
-- \\
-- \\
--\end{array}$ & $\begin{array}{l}-- \\
-- \\
-- \\
--\end{array}$ & $\begin{array}{l}70.6 \\
82.6 \\
63.2 \\
56.0 \\
71.0\end{array}$ & $\begin{array}{l}54.82 \\
56.42 \\
57.32 \\
58.02 \\
60.32\end{array}$ & $\begin{array}{l}728.62 \\
733.90 \\
743.71 \\
752.53 \\
770.24\end{array}$ \\
\hline VEM-308-47 & 1.8 & 5.5 & -- & -- & -- & 75.5 & 62.12 & 780.14 \\
\hline
\end{tabular}

\footnotetext{
${ }^{1}$ Fossil collection made by H. Wedow, Paleontology and Stratigraphy Branch, U. S. Geological Survey.
} 


\begin{tabular}{|c|c|c|c|c|c|c|c|c|c|c|}
\hline \multirow[b]{2}{*}{$\begin{array}{c}\text { Bed } \\
\text { no. }\end{array}$} & \multirow[b]{2}{*}{ Rock description } & \multirow{2}{*}{$\begin{array}{l}\text { Sample } \\
\text { no. }\end{array}$} & \multirow{2}{*}{$\begin{array}{l}\text { Thickness } \\
\text { (feet) }\end{array}$} & \multicolumn{5}{|c|}{ Chemical analyses (percent) } & \multirow{2}{*}{$\begin{array}{l}\text { Cumulative } \\
\text { Thick } \\
\text { (feet) }\end{array}$} & \multirow{2}{*}{$\begin{array}{l}\text { Thickness } \mathrm{x} \\
\text { percent } \mathrm{P}_{2} \mathrm{O}_{5} \\
\text { (cumulative) }\end{array}$} \\
\hline & & & & $\mathrm{P}_{2} \mathrm{O}_{5}$ & $\mathrm{Al}_{2} \mathrm{O}_{3}$ & $\mathrm{Fe}_{2} \mathrm{O}_{3}$ & $\begin{array}{l}\text { Loss on } \\
\text { ignition }\end{array}$ & $\begin{array}{l}\text { Acid } \\
\text { insoluble }\end{array}$ & & \\
\hline $\begin{array}{l}P-132 \\
P-131 \\
P-130 \\
P-129 \\
P-128\end{array}$ & $\begin{array}{l}\text { Mudstone } \\
\text { Mudstone } \\
\text { Mudstone } \\
\text { Mudstone, phosphatic } \\
\text { Phosphate rock, argillaceous }\end{array}$ & $\begin{array}{l}\text { VEM-307-47 } \\
\text { VEM-306-47 } \\
\text { VEM-305-47 } \\
\text { RAG- } 138-47 \\
\text { RAG- } 137-47\end{array}$ & $\begin{array}{l}1.7 \\
1.9 \\
2.0 \\
0.51 \\
0.65\end{array}$ & $\begin{array}{r}2.9 \\
2.2 \\
3.8 \\
8.7 \\
27.1\end{array}$ & $\begin{array}{l}-- \\
-- \\
-- \\
--\end{array}$ & $\begin{array}{l}-- \\
-- \\
-- \\
--\end{array}$ & $\begin{array}{l}-- \\
-- \\
-- \\
--\end{array}$ & $\begin{array}{l}83.6 \\
77.8 \\
81.1 \\
63.8 \\
26.9\end{array}$ & $\begin{array}{l}63.82 \\
65.72 \\
67.72 \\
68.23 \\
68.88\end{array}$ & $\begin{array}{l}785.07 \\
789.25 \\
796.85 \\
801.29 \\
818.89\end{array}$ \\
\hline $\begin{array}{l}P-127 \\
P-126 \\
P-125 \\
P-124 \\
P-123\end{array}$ & $\begin{array}{l}\text { Mudstone, phosphatic } \\
\text { Mudstone } \\
\text { Mudstone and phosphatic mudstone } \\
\text { Mudstone; fos, col. no. } 47-\mathrm{HW}-148 \\
\text { Mudstone }\end{array}$ & $\begin{array}{l}\text { RAG }-136-47 \\
\text { RAG }-135-47 \\
\text { RAG }-134-47 \\
\text { RAG- } 133-47 \\
\text { RAG- } 132-47\end{array}$ & $\begin{array}{l}1.02 \\
1.25 \\
0.4 \\
2.15 \\
0.6\end{array}$ & $\begin{array}{r}8.7 \\
6.6 \\
15.6 \\
6.5 \\
3.5\end{array}$ & $\begin{array}{l}-- \\
-- \\
-- \\
--\end{array}$ & $\begin{array}{l}-- \\
-- \\
-- \\
--\end{array}$ & $\begin{array}{l}-- \\
-- \\
-- \\
--\end{array}$ & $\begin{array}{l}64.3 \\
71.9 \\
49.2 \\
71.2 \\
71.4\end{array}$ & $\begin{array}{l}69.90 \\
71.15 \\
71.55 \\
73.70 \\
74.30\end{array}$ & $\begin{array}{l}827.75 \\
836.00 \\
842.24 \\
856.23 \\
858.33\end{array}$ \\
\hline$P-122$ & $\begin{array}{l}\text { Mudstone, phosphatic; fos. col. no. } \\
\text { 47-HW-149 }\end{array}$ & RAW- 24-47 & 1.1 & 12.3 & -- & -- & -- & 58.6 & 75.40 & 871.86 \\
\hline $\begin{array}{l}P-121 \\
P-120\end{array}$ & $\begin{array}{l}\text { Mudstone } \\
\text { Mudstone, phosphatio; fos. col. no. }\end{array}$ & RAW $-23-47$ & 1.0 & 7.1 & -- & -- & -- & 66.3 & 76.40 & 878.96 \\
\hline P-119 & $\begin{array}{l}47-H W-150 \\
\text { Mudstone, phosphatic; fos. col. no. }\end{array}$ & RAW- $22-47$ & 0.8 & 9.1 & -- & -- & -- & 65.2 & 77.20 & 886.24 \\
\hline$P-118$ & $\begin{array}{l}47-H W-150 \\
\text { Mudstone, phosphatic }\end{array}$ & $\begin{array}{l}\text { RAW- } 21-47 \\
\text { RAW- } 20-47\end{array}$ & $\begin{array}{l}0.3 \\
1.3\end{array}$ & $\begin{array}{l}17.9 \\
11.0\end{array}$ & -- & -- & -- & $\begin{array}{l}46.6 \\
54.6\end{array}$ & $\begin{array}{l}77.50 \\
78.80\end{array}$ & $\begin{array}{l}891.61 \\
905.91\end{array}$ \\
\hline $\begin{array}{l}P-117 \\
P-116 \\
P=115 \\
P-114 \\
P-113\end{array}$ & $\begin{array}{l}\text { Mudstone; fos. col. no. } 47-\mathrm{HW}-151 \\
\text { Mudstone, phosphatic } \\
\text { Mudstone } \\
\text { Mudstone } \\
\text { Mudstone }\end{array}$ & $\begin{array}{l}\text { RAW- } 19-47 \\
\text { RAW- } 18-47 \\
\text { FCA- 288-47 } \\
\text { FCA- } 287-47 \\
\text { FCA- 286-47 }\end{array}$ & $\begin{array}{l}1.2 \\
1.3 \\
1.2 \\
1.3 \\
0.9\end{array}$ & $\begin{array}{r}4.7 \\
14.9 \\
1.0 \\
3.4 \\
0.6\end{array}$ & $\begin{array}{l}-- \\
-- \\
-- \\
--\end{array}$ & $\begin{array}{l}-- \\
-- \\
-- \\
--\end{array}$ & $\begin{array}{l}-- \\
-- \\
-- \\
--\end{array}$ & $\begin{array}{l}73.2 \\
52.0 \\
28.0 \\
80.3 \\
90.5\end{array}$ & $\begin{array}{l}80.00 \\
81.30 \\
82.50 \\
83.80 \\
84.70\end{array}$ & $\begin{array}{l}911.55 \\
930.92 \\
932.12 \\
936.54 \\
937.08\end{array}$ \\
\hline $\begin{array}{l}P-112 \\
P-111 \\
P-110 \\
P-109 \\
P-108\end{array}$ & $\begin{array}{l}\text { Mudstone, phosphatic } \\
\text { Mudstone } \\
\text { Mudstone } \\
\text { Mudstone } \\
\text { Phosphate rock, argillaceous }\end{array}$ & $\begin{array}{l}\text { FCA- 285-47 } \\
\text { FCA- 284-47 } \\
\text { FCA- 283-47 } \\
\text { FCA- 282-47 } \\
\text { RMC- 81-47 }\end{array}$ & $\begin{array}{l}0.4 \\
1.7 \\
0.8 \\
1.0 \\
0.3\end{array}$ & $\begin{array}{r}17.3 \\
3.0 \\
4.7 \\
0.8 \\
29.1\end{array}$ & $\begin{array}{l}-- \\
-- \\
-- \\
--\end{array}$ & $\begin{array}{l}-- \\
-- \\
-- \\
--\end{array}$ & $\begin{array}{l}-- \\
-- \\
-- \\
--\end{array}$ & $\begin{array}{l}45.1 \\
82.5 \\
76.7 \\
89.8 \\
20.0\end{array}$ & $\begin{array}{l}85.10 \\
86.80 \\
87.60 \\
88.60 \\
88.90\end{array}$ & $\begin{array}{l}944.00 \\
949.1 .0 \\
952.86 \\
953.66 \\
962.39\end{array}$ \\
\hline $\begin{array}{l}P-107 \\
P-106 \\
P-105 \\
P-104 \\
P-103\end{array}$ & $\begin{array}{l}\text { Mudstone } \\
\text { Phosphate rock, argillaceous } \\
\text { Phosphate rock, argillaceous } \\
\text { Mudstone, phosphatic } \\
\text { Mudstone; fos. col. no. 47-HW-136 }\end{array}$ & $\begin{array}{l}\text { RMC- } 80-47 \\
\text { RAG- } 106-47 \\
\text { RAG- } 105-47 \\
\text { RAG- } 104-47 \\
\text { RAG- } 103-47\end{array}$ & $\begin{array}{l}0.75 \\
0.3 \\
0.8 \\
0.4 \\
0.75\end{array}$ & $\begin{array}{r}3.7 \\
23.6 \\
0.2 \\
8.0 \\
0.4\end{array}$ & $\begin{array}{l}-- \\
-- \\
-- \\
--\end{array}$ & $\begin{array}{l}-- \\
-- \\
-- \\
--\end{array}$ & $\begin{array}{l}-- \\
-- \\
-- \\
--\end{array}$ & $\begin{array}{l}78.3 \\
32.8 \\
91.4 \\
69.4 \\
90.0\end{array}$ & $\begin{array}{l}89.65 \\
89.95 \\
90.75 \\
91.15 \\
91.90\end{array}$ & $\begin{array}{l}965.17 \\
972.25 \\
972.41 \\
975.61 \\
975.91\end{array}$ \\
\hline $\begin{array}{l}P-102 \\
P=101 \\
P-100 \\
P-99 \\
P-98\end{array}$ & $\begin{array}{l}\text { Mudstone, phosphatic } \\
\text { Mudstone } \\
\text { Phosphate rock } \\
\text { Mudstone and phosphatic mudstone } \\
\text { Phosphate rock }\end{array}$ & $\begin{array}{l}\text { RAG- } 102-47 \\
\text { RAG- } 101-47 \\
\text { RAG- } 100-47 \\
\text { RAG- } 99-47 \\
\text { VEM-288-47 }\end{array}$ & $\begin{array}{l}0.75 \\
1.05 \\
0.9 \\
0.85 \\
0.9\end{array}$ & $\begin{array}{r}11.0 \\
2.8 \\
32.3 \\
9.1 \\
33.5\end{array}$ & $\begin{array}{l}-- \\
-- \\
-- \\
-- \\
--\end{array}$ & $\begin{array}{l}-- \\
-- \\
-- \\
--\end{array}$ & $\begin{array}{l}-- \\
-- \\
-- \\
--\end{array}$ & $\begin{array}{r}62.6 \\
82.0 \\
12.2 \\
69.4 \\
9.9\end{array}$ & $\begin{array}{l}92.65 \\
93.70 \\
94.60 \\
95.45 \\
96.35\end{array}$ & $\begin{array}{r}984.16 \\
987.10 \\
1,016.17 \\
1,023.91 \\
1,054.06\end{array}$ \\
\hline $\begin{array}{l}P=97 \\
P=96 \\
P=95\end{array}$ & $\begin{array}{l}\text { Mudstone } \\
\text { Mudstone and phosphate rock } \\
\text { Phosphate rack, cherty }\end{array}$ & $\begin{array}{l}\text { VEM-287-47 } \\
\text { VEM-286-47 } \\
\text { VEM-285-47 }\end{array}$ & $\begin{array}{l}3.8 \\
0.8 \\
1.3\end{array}$ & $\begin{array}{r}0.9 \\
10.6 \\
27.0\end{array}$ & -- & $\begin{array}{l}-- \\
--\end{array}$ & $\begin{array}{l}-- \\
--\end{array}$ & $\begin{array}{l}89.4 \\
62.3 \\
25.8\end{array}$ & $\begin{array}{l}100.15 \\
100.95 \\
102.25\end{array}$ & $\begin{array}{l}1,057.48 \\
1,065.96 \\
1,069.47\end{array}$ \\
\hline
\end{tabular}




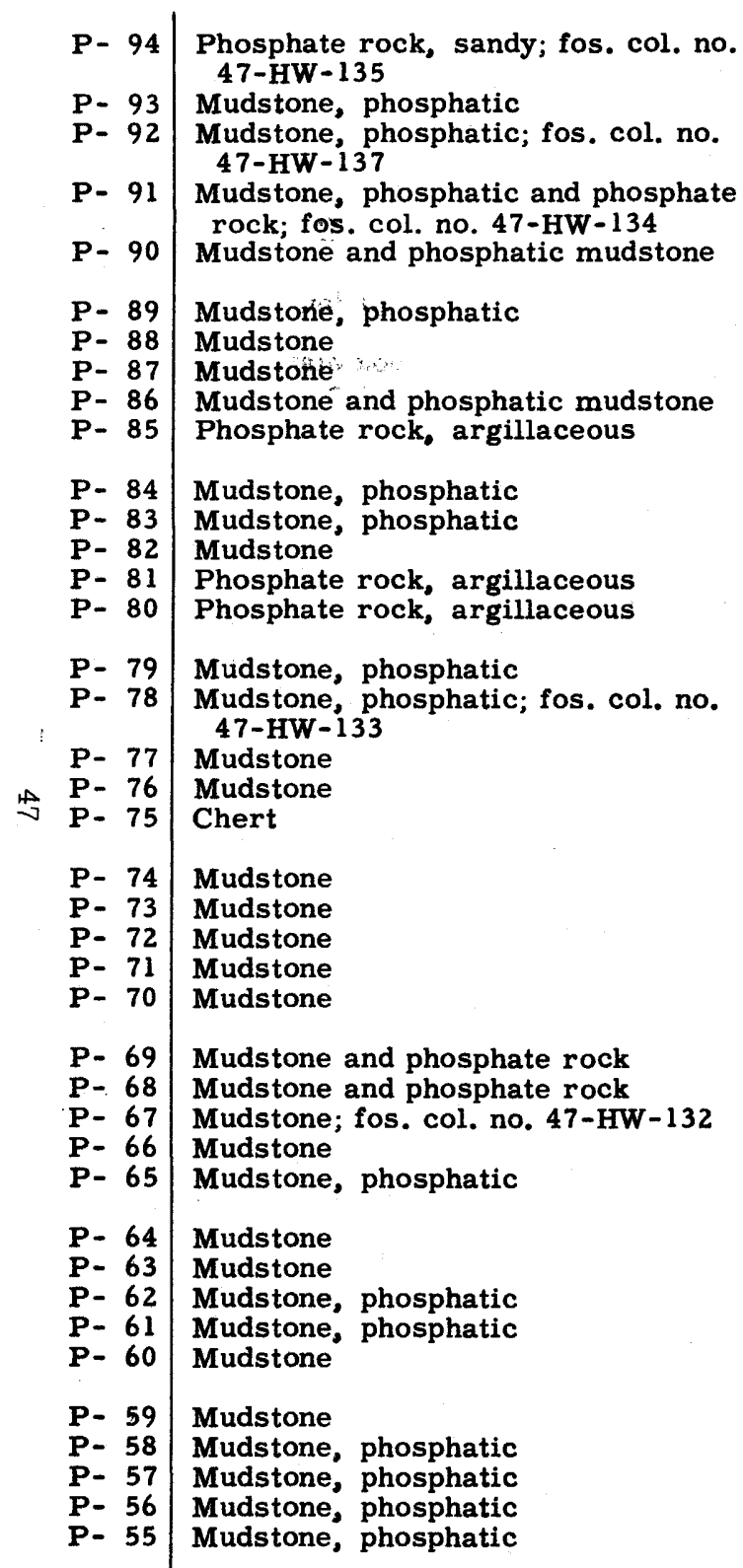

114.50

115.95

116.23

116.58

117.21

117.96

74.3

73.8

71.8

119.76

120.45

122.05

76.9

59.2

122.68

123.48

124.88

\begin{tabular}{l|l}
77.2 & 125.38 \\
61.8 & 125.88
\end{tabular}

85. 5

86.3

126.88

127.58

129.28

\begin{tabular}{l|l}
52.7 & 130.68 \\
74.8 & 131.53
\end{tabular}

68.5

132.23

132.73

63.4

56.5

45.6
53.7

$1,098.02$

$1,102.22$

$1,104.37$

$1,106.32$

$1,106.96$

$1,114.66$

$1,135.54$

$1,141.59$

$1,155.18$

$1,159.41$

$1,165.49$

1. 173.37

$1,198.03$

$1,214.51$

$1,220.01$

$1,220.15$

$1,220.40$

$1,221.72$

1. 224.80

1. 231.10

$1,233.92$

1. 235.68

$1,237.89$

$1,244.29$

$1,248.77$

$1,250.97$

1, 255. 32

$1,256.52$

1, 256. 94

$1,271.73$

$1,288.53$

$1,292.44$

1. 296.36 $1,300.71$

133.73

135.73

$1,311.71$

$1,341.91$
$1,354.56$ 


\begin{tabular}{|c|c|c|c|c|c|c|c|c|c|c|}
\hline \multirow[b]{2}{*}{$\begin{array}{c}\text { Bed } \\
\text { no. }\end{array}$} & \multirow[b]{2}{*}{ Rock description } & \multirow{2}{*}{$\begin{array}{c}\text { Sample } \\
\text { no. }\end{array}$} & \multirow{2}{*}{$\begin{array}{c}\text { Thickness } \\
\text { (feet) }\end{array}$} & \multicolumn{5}{|c|}{ Chemical analyses (percent) } & \multirow{2}{*}{$\begin{array}{l}\text { Cumulative } \\
\text { thickness } \\
\text { (feet) }\end{array}$} & \multirow{2}{*}{$\begin{array}{l}\text { Thickness } x \\
\text { percent } \mathrm{P}_{2} \mathrm{O}_{5} \\
\text { (cumulative) }\end{array}$} \\
\hline & & & & $\mathrm{P}_{2} \mathrm{O}_{5}$ & $\mathrm{Al}_{2} \mathrm{O}_{3}$ & $\mathrm{Fe}_{2} \mathrm{O}_{3}$ & $\begin{array}{l}\text { Loss on } \\
\text { ignition }\end{array}$ & $\begin{array}{c}\text { Acid } \\
\text { insoluble }\end{array}$ & & \\
\hline $\begin{array}{l}P-54 \\
P-53 \\
P-52 \\
P-51 \\
P-50\end{array}$ & $\begin{array}{l}\text { Mudstone, phosphatic } \\
\text { Mudstone, phosphatic } \\
\text { Mudstone, phosphatic } \\
\text { Mudstone, phosphatic } \\
\text { Mudstone, phosphatic }\end{array}$ & $\begin{array}{l}\text { VEM-284-47 } \\
\text { VEM-283-47 } \\
\text { VEM-282-47 } \\
\text { VEM-281-47 } \\
\text { VEM-280-47 }\end{array}$ & $\begin{array}{l}1.4 \\
1.1 \\
1.5 \\
2.0 \\
2.0\end{array}$ & $\begin{array}{l}11.9 \\
12.4 \\
14.7 \\
11.2 \\
13.5\end{array}$ & $\begin{array}{l}-- \\
-- \\
-- \\
--\end{array}$ & $\begin{array}{l}-- \\
-- \\
-- \\
--\end{array}$ & $\begin{array}{l}-- \\
-- \\
-- \\
--\end{array}$ & $\begin{array}{l}55.0 \\
53.0 \\
46.9 \\
56.1 \\
50.3\end{array}$ & $\begin{array}{l}138.23 \\
139.33 \\
140.83 \\
142.83 \\
144.83\end{array}$ & $\begin{array}{l}1,371.22 \\
1,384.86 \\
1,406.91 \\
1,429.31 \\
1,456.31\end{array}$ \\
\hline $\begin{array}{l}P=49 \\
P-48 \\
P=47 \\
P=46 \\
P=45\end{array}$ & $\begin{array}{l}\text { Phosphate rock, argillaceous } \\
\text { Phosphate rock } \\
\text { Phosphate rock } \\
\text { Phosphate rock } \\
\text { Phosphate rock, argillaceous }\end{array}$ & $\begin{array}{l}\text { VEM-279-47 } \\
\text { VEM-278-47 } \\
\text { VEM-277-47 } \\
\text { VEM-276-47 } \\
\text { VEM-275-47 }\end{array}$ & $\begin{array}{l}0.9 \\
0.8 \\
0.7 \\
0.5 \\
0.3\end{array}$ & $\begin{array}{l}21.6 \\
28.1 \\
31.4 \\
35.3 \\
27.6\end{array}$ & $\begin{array}{l}7.5 \\
5.2 \\
1.1 \\
2.0 \\
4.4\end{array}$ & $\begin{array}{l}2.83 \\
1.75 \\
0.15 \\
1.13 \\
2.60\end{array}$ & $\begin{array}{l}5.06 \\
3.94 \\
3.22 \\
2.20 \\
4.18\end{array}$ & $\begin{array}{r}34.7 \\
19.4 \\
14.2 \\
6.7 \\
20.9\end{array}$ & $\begin{array}{l}145.73 \\
146.53 \\
147.23 \\
147.73 \\
148.03\end{array}$ & $\begin{array}{l}1,475.75 \\
1,498.23 \\
1,520.21 \\
1,537.86 \\
1,546.14\end{array}$ \\
\hline $\begin{array}{l}P=44 \\
P-43 \\
P-42 \\
P=41 \\
P=40\end{array}$ & $\begin{array}{l}\text { Phosphate rock and mudstone } \\
\text { Phosphate rock } \\
\text { Phosphate rock } \\
\text { Phosphate rock and mudstone } \\
\text { Phosphate rock }\end{array}$ & $\begin{array}{l}\text { LES- } 425-47 \\
\text { LES- } 424-47 \\
\text { LES- } 423-47 \\
\text { LES- } 422-47 \\
\text { LES- } 421-47\end{array}$ & $\begin{array}{l}0.75 \\
0.5 \\
0.8 \\
1.0 \\
1.15\end{array}$ & $\begin{array}{l}18.6 \\
31.6 \\
33.5 \\
24.0 \\
30.8\end{array}$ & $\begin{array}{l}7.8 \\
2.4 \\
2.7 \\
7.3 \\
1.6\end{array}$ & $\begin{array}{l}3.31 \\
1.66 \\
1.2 \\
1.99 \\
1.28\end{array}$ & $\begin{array}{l}6.38 \\
5.02 \\
3.02 \\
6.34 \\
3.94\end{array}$ & $\begin{array}{l}38.3 \\
12.1 \\
10.6 \\
27.9 \\
16.2\end{array}$ & $\begin{array}{l}148.78 \\
149.28 \\
150.08 \\
151.08 \\
152.23\end{array}$ & $\begin{array}{l}1,560.09 \\
1,575.89 \\
1,602.69 \\
1,626.69 \\
1,662.11\end{array}$ \\
\hline $\begin{array}{l}P-39 \\
P-38 \\
P-37 \\
P-36 \\
P-35\end{array}$ & $\begin{array}{l}\text { Mudstone, phosphatic, and argillaceous } \\
\text { phosphate rock } \\
\text { Phosphate rock and phosphatic mudstone } \\
\text { Mudstone, phosphatic } \\
\text { Mudstone, phosphatic } \\
\text { Mudstone, phosphatic }\end{array}$ & $\begin{array}{l}\text { LES- } 420-47 \\
\text { LES- } 419-47 \\
\text { LES- } 418-47 \\
\text { LES- } 417-47 \\
\text { LES- } 416-47\end{array}$ & $\begin{array}{l}0.85 \\
0.5 \\
0.45 \\
0.9 \\
2.2\end{array}$ & $\begin{array}{l}17.6 \\
21.3 \\
16.4 \\
11.4 \\
13.8\end{array}$ & $\begin{array}{l}7.1 \\
4.7 \\
6.7 \\
-. \\
--\end{array}$ & $\begin{array}{r}2.55 \\
2.34 \\
2.52 \\
-- \\
--\end{array}$ & $\begin{array}{l}7.06 \\
6.68 \\
6.28 \\
-. \\
--\end{array}$ & $\begin{array}{l}41.8 \\
33.4 \\
48.1 \\
57.5 \\
50.8\end{array}$ & $\begin{array}{l}153.08 \\
153.58 \\
154.03 \\
154.93 \\
157.13\end{array}$ & $\begin{array}{l}1,677.06 \\
1,687.71 \\
1,695.09 \\
1,705.35 \\
1,735.71\end{array}$ \\
\hline $\begin{array}{l}P-34 \\
P-33 \\
P-32 \\
P-31 \\
P-30\end{array}$ & $\begin{array}{l}\text { Mudstone, phosphatic; fos. col. no. } \\
47-\text { HW-131 } \\
\text { Mudstone, phosphatic } \\
\text { Mudstone, phosphatic } \\
\text { Mudstone } \\
\text { Mudstone, phosphatic }\end{array}$ & $\begin{array}{l}\text { LES- 415-47 } \\
\text { LES- } 414-47 \\
\text { LES- } 413-47 \\
\text { LES- } 412-47 \\
\text { LES- } 411-47\end{array}$ & $\begin{array}{l}0.45 \\
0.65 \\
0.65 \\
0.5 \\
0.3\end{array}$ & $\begin{array}{r}10.4 \\
14.4 \\
7.8 \\
2.7 \\
15.2\end{array}$ & $\begin{array}{l}-- \\
-- \\
-- \\
--\end{array}$ & $\begin{array}{l}-- \\
-- \\
-- \\
--\end{array}$ & $\begin{array}{l}-- \\
-- \\
-- \\
--\end{array}$ & $\begin{array}{l}56.9 \\
49.3 \\
64.4 \\
77.2 \\
46.8\end{array}$ & $\begin{array}{l}157.58 \\
158.23 \\
158.88 \\
159.38 \\
159.68\end{array}$ & $\begin{array}{l}1,740.39 \\
1,749.75 \\
1,754.82 \\
1,756.17 \\
1,760.73\end{array}$ \\
\hline $\begin{array}{l}P-29 \\
P-28 \\
P-27 \\
P-26 \\
P-25\end{array}$ & $\begin{array}{l}\text { Phosphate rock and mudstone; fos. } \\
\text { col. no. } 47-\mathrm{HW}-138 \\
\text { Phosphate rock and mudstone } \\
\text { Phosphate rock } \\
\text { Phosphate rock, argillaceous } \\
\text { Phosphate rock, argillaceous }\end{array}$ & $\begin{array}{l}\text { RMC- } 63-47 \\
\text { RMC- } 62-47 \\
\text { RMC- } 61-47 \\
\text { FCA- 250-47 } \\
\text { FCA- 249-47 }\end{array}$ & $\begin{array}{l}0.9 \\
1.3 \\
0.75 \\
0.4 \\
0.9\end{array}$ & $\begin{array}{l}22.2 \\
28.9 \\
29.5 \\
25.6 \\
27.2\end{array}$ & $\begin{array}{l}4.3 \\
0.66 \\
2.8 \\
4.2 \\
3.6\end{array}$ & $\begin{array}{l}1.50 \\
0.28 \\
0.83 \\
1.29 \\
1.42\end{array}$ & $\begin{array}{l}5.86 \\
5.04 \\
4.64 \\
4.78 \\
4.78\end{array}$ & $\begin{array}{l}32.5 \\
17.3 \\
17.6 \\
26.5 \\
23.7\end{array}$ & $\begin{array}{l}160.58 \\
161.88 \\
162.63 \\
163.03 \\
163.93\end{array}$ & $\begin{array}{l}1,780.71 \\
1,818.28 \\
1,840.43 \\
1,850.67 \\
1,875.15\end{array}$ \\
\hline $\begin{array}{l}P=24 \\
P=23 \\
P=22 \\
P=21 \\
P=20\end{array}$ & $\begin{array}{l}\text { Mudstone, phosphatic } \\
\text { Phosphate rock } \\
\text { Phosphate rock, argillaceous } \\
\text { Phosphate rock } \\
\text { Mudstone, phosphatic }\end{array}$ & $\begin{array}{l}\text { FCA- 248-47 } \\
\text { FCA- 247-47 } \\
\text { FCA- 246-47 } \\
\text { RAG- } 90-47 \\
\text { RAG- } 89-47\end{array}$ & $\begin{array}{l}1.3 \\
1.25 \\
1.05 \\
1.75 \\
0.88\end{array}$ & $\begin{array}{l}14.2 \\
29.2 \\
26.7 \\
28.5 \\
15.1\end{array}$ & $\begin{array}{l}7.8 \\
2.7 \\
3.6 \\
6.7 \\
2.4\end{array}$ & $\begin{array}{l}1.99 \\
1.12 \\
1.26 \\
0.70 \\
1.75\end{array}$ & $\begin{array}{l}6.90 \\
6.34 \\
6.34 \\
7.26 \\
6.70\end{array}$ & $\begin{array}{l}51.4 \\
17.0 \\
24.4 \\
17.6 \\
50.6\end{array}$ & $\begin{array}{l}165.23 \\
166.48 \\
167.53 \\
169.28 \\
170.16\end{array}$ & $\begin{array}{l}1,893.61 \\
1,928.65 \\
1,956.65 \\
2,006.65 \\
2,019.94\end{array}$ \\
\hline $\begin{array}{l}P-19 \\
P-18 \\
P=17 \\
P=16\end{array}$ & $\begin{array}{l}\text { Phosphate rock and mudstone } \\
\text { Phosphate rock, argillaceous } \\
\text { Phosphate rock, argillaceous } \\
\text { Phosphate rock, argillaceous }\end{array}$ & $\begin{array}{l}\text { RAG- } 88-47 \\
\text { RAG- } 87-47 \\
\text { RAG- } 86-47 \\
\text { RAG- } 85-47\end{array}$ & $\begin{array}{l}1.2 \\
1.5 \\
0.6 \\
0.52\end{array}$ & $\begin{array}{l}25.0 \\
25.1 \\
22.7 \\
24.5\end{array}$ & $\begin{array}{l}4.7 \\
5.5 \\
4.1 \\
3.9\end{array}$ & $\begin{array}{l}1.24 \\
1.28 \\
1.37 \\
1.52\end{array}$ & $\begin{array}{l}6.08 \\
7.14 \\
4.46 \\
4.40\end{array}$ & $\begin{array}{l}26.4 \\
24.8 \\
34.5 \\
31.0\end{array}$ & $\begin{array}{l}171.36 \\
172.86 \\
173.46 \\
173.98\end{array}$ & $\begin{array}{l}2,049.94 \\
2,087.59 \\
2,101.21 \\
2,113.96\end{array}$ \\
\hline
\end{tabular}




\begin{tabular}{|c|c|c|c|c|c|c|c|c|c|c|}
\hline $\begin{array}{l}P-15 \\
P-14 \\
P=13 \\
P-12 \\
P-11\end{array}$ & $\begin{array}{l}\text { Phosphate rock } \\
\text { Phosphate rock } \\
\text { Phosphate rock and phosphatic mudstone } \\
\text { Phosphate rock } \\
\text { Phosphate rock }\end{array}$ & $\begin{array}{l}\text { RAG- } 84-47 \\
\text { RAG- } 83-47 \\
\text { RAG- } 82-47 \\
\text { RAG- } 81-47 \\
\text { RAG- } 80-47\end{array}$ & $\begin{array}{l}0.66 \\
1.2 \\
0.9 \\
0.97 \\
0.92\end{array}$ & $\begin{array}{l}30.1 \\
32.1 \\
31.6 \\
31.7 \\
32.1\end{array}$ & $\begin{array}{l}2.5 \\
2.8 \\
1.8 \\
1.9 \\
2.4\end{array}$ & $\begin{array}{l}1.13 \\
0.91 \\
0.91 \\
0.89 \\
0.93\end{array}$ & $\begin{array}{l}5.84 \\
6.50 \\
6.56 \\
6.28 \\
6.12\end{array}$ & $\begin{array}{r}14.5 \\
9.4 \\
9.9 \\
9.1 \\
8.1\end{array}$ & $\begin{array}{l}174.64 \\
175.84 \\
176.74 \\
177.71 \\
178.63\end{array}$ & $\begin{array}{l}2,133.86 \\
2,172.38 \\
2,200.82 \\
2,232.52 \\
2,262.05\end{array}$ \\
\hline $\begin{array}{l}P-10 \\
P-\quad 9 \\
P- \\
P-\quad 7 \\
P-\quad 6\end{array}$ & $\begin{array}{l}\text { Phosphate rock } \\
\text { Phosphate rock } \\
\text { Phosphate rock, argillaceous } \\
\text { Phosphate rock } \\
\text { Mudstone }\end{array}$ & $\begin{array}{l}\text { RAG- } 79-47 \\
\text { RAG- } 78-47 \\
\text { LES- } 410-47 \\
\text { LES- } 409-47 \\
\text { LES- } 408-47\end{array}$ & $\begin{array}{l}1.05 \\
1.2 \\
0.35 \\
0.6 \\
0.6\end{array}$ & $\begin{array}{r}34.3 \\
33.6 \\
25.8 \\
27.6 \\
4.3\end{array}$ & $\begin{array}{l}1.3 \\
1.0 \\
3.3 \\
2.0 \\
-.\end{array}$ & $\begin{array}{l}0.71 \\
0.67 \\
1.24 \\
0.84 \\
--\end{array}$ & $\begin{array}{l}5.76 \\
5.78 \\
5.50 \\
5.82 \\
--\end{array}$ & $\begin{array}{r}4.3 \\
6.0 \\
22.2 \\
18.9 \\
65.2\end{array}$ & $\begin{array}{l}179.68 \\
180.88 \\
181.23 \\
181.83 \\
182.43\end{array}$ & $\begin{array}{l}2,298.05 \\
2,338.37 \\
2,347.41 \\
2,363.97 \\
2,366.55\end{array}$ \\
\hline 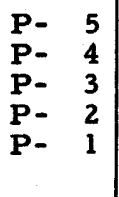 & $\begin{array}{l}\text { Mudstone, phosphatic } \\
\text { Mudstone } \\
\text { Mudstone } \\
\text { Mudstone } \\
\text { Phosphate rock and phosphatic mudstone; } \\
\text { fos. col. no. } 47-\mathrm{HW}-130\end{array}$ & $\begin{array}{l}\text { LES- } 407-47 \\
\text { LES- } 406-47 \\
\text { LES- } 405-47 \\
\text { LES- } 404-47 \\
\text { LES- } 403-47\end{array}$ & $\begin{array}{l}0.6 \\
1.5 \\
1.1 \\
1.2 \\
0.4\end{array}$ & $\begin{array}{l}8.9 \\
1.5 \\
1.2 \\
2.3 \\
34.7\end{array}$ & $\begin{array}{l}-- \\
-- \\
-- \\
--\end{array}$ & $\begin{array}{l}-- \\
-- \\
--\end{array}$ & $\begin{array}{l}-- \\
-- \\
-- \\
--\end{array}$ & $\begin{array}{l}58.7 \\
79.2 \\
79.8 \\
76.1 \\
6.5\end{array}$ & $\begin{array}{l}183.03 \\
184.53 \\
185.63 \\
186.83 \\
187.23\end{array}$ & $\begin{array}{l}2,371.89 \\
2,374.14 \\
2,375.46 \\
2,378.22 \\
2,392.10\end{array}$ \\
\hline \multicolumn{11}{|c|}{ Wells formation } \\
\hline $\begin{array}{l}C w-12 \\
C w-11 \\
C w-10 \\
C w-9 \\
C w-8\end{array}$ & $\begin{array}{l}\text { Mudstone, contains limestone nodules } \\
\text { Limestone; fos. col. no. } 47-\mathrm{HW}-126 \\
\text { Limestone; fos. col. no. } 47-\mathrm{HW}-127 \\
\text { Limestone and mudstone; fos. col. no. } \\
47-\mathrm{HW}-128 \\
\text { Limestone; fos. col. no. } 47-\mathrm{HW}-129\end{array}$ & $\begin{array}{c}\text { LES- } 402-47 \\
\text { LES- } 401-47 \\
-- \\
-- \\
--\end{array}$ & $\begin{array}{l}0.3 \\
2.8 \\
0.8 \\
2.0 \\
3.3\end{array}$ & $\begin{array}{l}5.4 \\
0.7 \\
-- \\
-- \\
--\end{array}$ & $\begin{array}{l}-- \\
-- \\
-- \\
--\end{array}$ & $\begin{array}{l}-- \\
-- \\
-- \\
--\end{array}$ & $\begin{array}{l}-- \\
-- \\
-- \\
--\end{array}$ & $\begin{array}{c}46.8 \\
5.0 \\
-- \\
-- \\
--\end{array}$ & $\begin{array}{l}0.3 \\
3.1 \\
3.9 \\
5.9 \\
9.2\end{array}$ & $\begin{array}{l}1.62 \\
3.58 \\
-- \\
-- \\
--\end{array}$ \\
\hline$C w-8$ & Limestone; Ios. col. no. 47-HW-129 & - & 3.3 & -- & -- & -- & -- & -- & 9.2 & - \\
\hline $\begin{array}{l}C w-7 \\
C w-6 \\
C w-5 \\
C w-4 \\
C w-3\end{array}$ & $\begin{array}{l}\text { Chert } \\
\text { Limestone } \\
\text { Limestone } \\
\text { Limestone } \\
\text { Limestone }\end{array}$ & $\begin{array}{l}-- \\
-- \\
-- \\
-- \\
--\end{array}$ & $\begin{array}{l}0.9 \\
4.5 \\
3.0 \\
1.8 \\
0.5\end{array}$ & $\begin{array}{l}-- \\
-- \\
-- \\
--\end{array}$ & $\begin{array}{l}-- \\
-- \\
-- \\
--\end{array}$ & $\begin{array}{l}-- \\
-- \\
-- \\
--\end{array}$ & $\begin{array}{l}-- \\
-- \\
-- \\
-- \\
--\end{array}$ & $\begin{array}{l}-- \\
-- \\
-- \\
--\end{array}$ & $\begin{array}{l}10.1 \\
14.6 \\
17.6 \\
19.4 \\
19.9\end{array}$ & $\begin{array}{l}-- \\
-- \\
-- \\
-- \\
--\end{array}$ \\
\hline $\begin{array}{l}C w-2 \\
C w-1\end{array}$ & $\begin{array}{l}\text { Chert } \\
\text { Limestone }\end{array}$ & -- & 0.7 & -- & -- & -- & -- & -- & $\begin{array}{c}20.6 \\
-\end{array}$ & -- \\
\hline
\end{tabular}


MONTPELIER CANYON, IDAHO. LOT NOS. 1207 AND 1236.

Phosphoria formation measured and sampled in hand trenches on westward-dipping fault block, northwest side of Montpelier Canyon three miles east of Montpelier, sec. 31, T. 12 S., R. 45 E., Bear Lake County, Idaho. Most of section (lot no. 1207) measured in long trench, exposing strata that strike N. $18^{\circ}$ E. and dip $29^{\circ}$ W., by F. C. Armstrong, R. M. Campbell, R. A. Gulbrandsen, and R. A. Hoppin and sampled by Campbell, O. A. Payne, R. A. Smart, and R. S. Sears during August 1947. Lower part of phosphatic shale (lot no. 1236) measured in two trenches 425 feet to the southwest exposing strata that strike N. $32^{\circ}$ E. and dip $30^{\circ}$ NW., by R. A. Gulbrandsen and R.P. Sheldon and sampled by R. A. Smart in September 1948 . Samples analyzed for $\mathrm{P}_{2} \mathrm{O}_{5}, \mathrm{~F}$, and acid insoluble by U. S. Bureau of Mines Laboratory, Albany, Oregon, and for other constituents by Trace Elements Laboratory, U. S.' Geological Survey, Washington, D. C.

\begin{tabular}{|c|c|c|c|c|c|c|c|c|c|c|c|}
\hline \multirow{2}{*}{$\begin{array}{c}\text { Bed } \\
\text { no. }\end{array}$} & \multirow[b]{2}{*}{ Rock desçription } & \multirow{2}{*}{$\begin{array}{c}\text { Sample } \\
\text { no. }\end{array}$} & \multirow{2}{*}{$\begin{array}{c}\text { Thickness } \\
\text { (feet) }\end{array}$} & \multicolumn{6}{|c|}{ Chemical analyses (percent) } & \multirow{2}{*}{$\begin{array}{l}\text { Cumulative } \\
\text { thickness } \\
\text { (feet) }\end{array}$} & \multirow{2}{*}{$\begin{array}{l}\text { Thickness } \mathbf{x} \\
\text { percent } \mathbf{P}_{2} \mathrm{O}_{5} \\
\text { (cumulative) }\end{array}$} \\
\hline & & & & $\mathrm{P}_{2} \mathrm{O}_{5}$ & $\mathrm{Al}_{2} \mathrm{O}_{3}$ & $\mathrm{Fe}_{2} \mathrm{O}_{3}$ & $F$ & $\begin{array}{l}\text { Loss on } \\
\text { ignition }\end{array}$ & $\begin{array}{c}\text { Acid } \\
\text { insoluble }\end{array}$ & & \\
\hline
\end{tabular}

Dinwoody formation—basal bed only-lot no. 1207

Trd- 1 Limestone, argillaceous; fos. col. no. 47-HW-187

RAH-242-47

4.0

0.8

-

-

$-$

46.8

4.0

3.20

\begin{tabular}{lr|l}
\hline $\mathrm{U}-12$ & $\begin{array}{l}\text { Chert, phosphatic; fos. col. } \\
\text { no. } 4 \text { 7-HW-186 }\end{array}$ \\
$\mathrm{U}-11$ & Limestone, argillaceous \\
$\mathrm{U}-10$ & Mudstone, calcareous \\
$\mathrm{U}-$ & 9 & Mudstone \\
$\mathrm{U}-$ & 8 & Mudstone \\
$\mathrm{U}-$ & 7 & Mudstone \\
$\mathrm{U}-$ & 6 & Limestone, argillaceous \\
$\mathrm{U}-$ & 5 & Mudstone \\
$\mathrm{U}-$ & 4 & Limestone, argillaceous \\
$\mathrm{U}-$ & 3 & Chert \\
$\mathrm{U}-$ & 2 & Chert, calcareous \\
$\mathrm{U}-$ & 1 & Mudstone
\end{tabular}

Upper shale member of Phosphoria formation-lot no. 1207

\begin{tabular}{|l|l|l|l|l|l|l} 
& & & & & & \\
RAH-243-47 & 0.5 & 16.3 & -- & -- & -- & - \\
RAH-244-47 & 2.1 & 1.6 & -- & -- & -- & - \\
RAH-245-47 & 2.9 & 0.7 & -- & -- & -- & - \\
RAH-246-47 & 3.4 & 1.4 & -- & -- & -- & - \\
RAH-247-47 & 1.2 & 1.5 & -- & -- & -- & - \\
RAH-248-47 & 1.6 & 2.4 & -- & -- & -- & - \\
RAH-249-47 & 1.3 & 1.2 & -- & -- & -- & - \\
RAH-250-47 & 2.6 & 1.3 & -- & -- & -- & - \\
RAH-251-47 & 2.6 & 0.9 & -- & -- & -- & - \\
RAH-252-47 & 2.1 & 1.4 & -- & -- & -- & - \\
RAH-253-47 & 4.7 & 1.5 & -- & -- & -- & - \\
RAH-254-47 & 5.0 & 2.9 & -- & -- & -- & -
\end{tabular}

\begin{tabular}{l|l|} 
& \\
-- & 44.6 \\
-- & 29.3 \\
-- & 52.4 \\
-- & 69.8 \\
-- & 68.0 \\
& 70.6 \\
-- & 40.8 \\
-- & 75.9 \\
-- & 37.9 \\
-- & 82.0 \\
& 61.1 \\
-- & 64.9 \\
\hline- &
\end{tabular}

0.5

2.6
5.5

5. 5

8.9
10.1

11.7

13.0

15.6

15.6
18.2

20.3

25.0
30.0

30.0

- lot no. 1207

Rex member of Phosphoria formation-lot no. 1207

\begin{tabular}{|c|c|}
\hline$R-27$ & $\begin{array}{l}\text { Limestone, cherty; fos. col. } \\
\text { no, 47-HW-185 }\end{array}$ \\
\hline$R-26$ & $\begin{array}{l}\text { Limestone, cherty; fos. col. } \\
\text { no. 47-HW-184 }\end{array}$ \\
\hline$R-25$ & $\begin{array}{l}\text { Chert, calcareous; fos. col. } \\
\text { no. } 47-\mathrm{HW}-183\end{array}$ \\
\hline $\begin{array}{l}R-24 \\
R-23\end{array}$ & $\begin{array}{l}\text { Chert, calcareous } \\
\text { Chert, calcareous }\end{array}$ \\
\hline $\begin{array}{l}\mathbf{R}-22 \\
\mathbf{R}-21\end{array}$ & $\begin{array}{l}\text { Chert, calcareous } \\
\text { Chert, calcareous }\end{array}$ \\
\hline
\end{tabular}

RAH-255-47

RAH-256-47

RAH-257-47

RAH $-258-47$

RAH -259-47

RAH $-260-47$

RAH $-261-47$
2.5
2.2
9.8
9.6
9.0
9.3
12.5

\begin{tabular}{|l|l|l|l|}
0.6 & -- & -- & -- \\
0.6 & -- & -- & -- \\
0.4 & -- & -- & -- \\
0.6 & -- & -- & -- \\
0.5 & -- & - & - \\
0.4 & -- & -- & -- \\
0.3 & -- & -- & --
\end{tabular}

--
--
--
--
--
--
--

35.4
45.9
64.4
75.2
71.1
76.6
68.6

2.5

4. 7

14.5

24. 1

33.1

42.4
8.15

11.51

13.54

18.30

20.10

23. 94

25.50

28.88

31.22

34.16

41.21

55. 71 


\begin{tabular}{|c|c|}
\hline $\begin{array}{l}R-20 \\
R-19 \\
R-18 \\
R-17 \\
R-16\end{array}$ & $\begin{array}{l}\text { Chert, calcareous } \\
\text { Chert, calcareous } \\
\text { Limestone, cherty } \\
\text { Limestone, cherty } \\
\text { Chert, calcareous }\end{array}$ \\
\hline $\begin{array}{l}R-15 \\
R-14 \\
R-13 \\
R-12 \\
R-11\end{array}$ & $\begin{array}{l}\text { Limestone, argillaceous } \\
\text { Chert and limestone } \\
\text { Chert, calcareous } \\
\text { Chert and limestone } \\
\text { Limestone and chert }\end{array}$ \\
\hline R- 10 & $\begin{array}{l}\text { Limestone and chert; fos. col. } \\
\text { no. } 47-\mathrm{HW}-182\end{array}$ \\
\hline $\begin{array}{ll}R- & 9 \\
R- & 8 \\
R- & 7 \\
R- & 6\end{array}$ & $\begin{array}{l}\text { Chert, calcareous } \\
\text { Limestone and chert } \\
\text { Chert and limestone } \\
\text { Limestone and chert }\end{array}$ \\
\hline $\begin{array}{ll}R- & 5 \\
R- & 4 \\
R- & 3 \\
R- & 2 \\
R- & 1\end{array}$ & $\begin{array}{l}\text { Chert, calcareous } \\
\text { Chert } \\
\text { Limestone, cherty } \\
\text { Limestone, argillaceous } \\
\text { Chert, calcareous }\end{array}$ \\
\hline
\end{tabular}

\begin{tabular}{|c|c|c|c|c|}
\hline $\begin{array}{l}\text { RAH-262-47 } \\
\text { RAH-263-47 } \\
\text { RAH-264-47 } \\
\text { RAH-265-47 } \\
\text { RAH-266-47 }\end{array}$ & $\begin{array}{r}7.0 \\
12.8 \\
4.0 \\
6.0 \\
8.9\end{array}$ & $\begin{array}{l}0.5 \\
0.8 \\
0.5 \\
0.5 \\
0.4\end{array}$ & $\begin{array}{l}-- \\
-- \\
-- \\
--\end{array}$ & $\begin{array}{l}-- \\
-- \\
-- \\
--\end{array}$ \\
\hline $\begin{array}{l}\text { RAH-267-47 } \\
\text { RAH-268-47 } \\
\text { RAH-269-47 } \\
\text { RAH-270-47 } \\
\text { RAH-271-47 }\end{array}$ & $\begin{array}{l}5.2 \\
4.4 \\
4.5 \\
3.5 \\
3.8\end{array}$ & $\begin{array}{l}0.5 \\
0.6 \\
0.3 \\
0.4 \\
0.3\end{array}$ & $\begin{array}{l}-- \\
-- \\
-- \\
-- \\
--\end{array}$ & $\begin{array}{l}-- \\
-- \\
-- \\
-- \\
--\end{array}$ \\
\hline $\begin{array}{l}\text { RAH-272-47 } \\
\text { RAH-273-47 } \\
\text { RAH- } 274-47 \\
\text { RAH-275-47 } \\
\text { RAG- } 51-47\end{array}$ & $\begin{array}{l}4.3 \\
3.0 \\
2.9 \\
5.0 \\
3.65\end{array}$ & $\begin{array}{l}0.4 \\
0.5 \\
0.4 \\
0.3 \\
0.4\end{array}$ & $\begin{array}{l}-- \\
-- \\
-- \\
-- \\
--\end{array}$ & $\begin{array}{l}-- \\
-- \\
-- \\
--\end{array}$ \\
\hline $\begin{array}{l}\text { RAG- } 64-47 \\
\text { RAG- } 63-47 \\
\text { RAG- } 62-47 \\
\text { FCA- } 209-47 \\
\text { FCA- } 210-47\end{array}$ & $\begin{array}{l}0.6 \\
0.35 \\
0.4 \\
1.0 \\
0.3\end{array}$ & $\begin{array}{l}0.4 \\
0.8 \\
0.5 \\
0.8 \\
0.9\end{array}$ & $\begin{array}{l}-- \\
-- \\
-- \\
-\end{array}$ & $\begin{array}{l}-- \\
-- \\
-- \\
-- \\
--\end{array}$ \\
\hline
\end{tabular}

\begin{tabular}{|c|c}
-- & - \\
-- & - \\
-- & - \\
-- & - \\
-- & -- \\
-- & - \\
-- & - \\
-- & -- \\
-- & - \\
& \\
-- & -- \\
-- & -- \\
-- & -- \\
-- & -- \\
-- & -- \\
-- & -- \\
-- & -- \\
-- & - \\
-- & - \\
- & -
\end{tabular}

\begin{tabular}{l|l} 
& \\
-- & 74.7 \\
-- & 76.8 \\
-- & 42.1 \\
-- & 47.4 \\
& 70.0 \\
-- & 33.5 \\
-- & 67.0 \\
-- & 53.5 \\
-- & 48.6 \\
-- & 46.8 \\
& \\
-- & 33.4 \\
-- & 73.4 \\
-- & 50.6 \\
-- & 53.7 \\
-- & 36.8 \\
& \\
-- & 69.2 \\
-- & 70.5 \\
-- & 38.6 \\
-- & 21.2 \\
-- & 76.1
\end{tabular}

61.9
74.7
78.7
84.7
93.6
98.8
103.2
107.7
111.2
115.0

119.3
122.3
125.2
130.2
133.85
134.45
134.80
135.20
136.20
136.50

Phosphatic shale member of Phosphoria formation-lot nos. 1207 and 1236

\begin{tabular}{|l|l|l|l|l|l|l|l|} 
& & & & & & & \\
FCA-211-47 & 0.9 & 21.2 & -- & -- & -- & -- & 32.4 \\
FCA-212-47 & 1.6 & 1.2 & -- & -- & -- & -- & 68.7 \\
FCA-213-47 & 1.1 & 2.5 & -- & -- & -- & -- & 64.6 \\
FCA-214-47 & 0.7 & 6.3 & -- & -- & -- & -- & 57.5 \\
FCA-215-47 & 0.8 & 3.7 & -- & -- & -- & -- & 65.9 \\
FCA-216-47 & 2.8 & 0.7 & -- & -- & -- & -- & 79.5 \\
FCA-217-47 & 0.8 & 1.3 & -- & -- & -- & -- & 75.8 \\
FCA-208-47 & 0.6 & 0.5 & -- & -- & -- & -- & 67.7 \\
FCA-207-47 & 0.6 & 4.8 & -- & -- & -- & -- & 58.8 \\
FCA-206-47 & 1.2 & 2.4 & -- & -- & -- & -- & 66.7 \\
FCA-205-47 & 1.8 & 3.7 & -- & -- & -- & -- & 14.9 \\
FCA-204-47 & 0.3 & 16.6 & -- & -- & -- & -- & 37.7 \\
FCA-203-47 & 1.5 & 5.1 & -- & -- & -- & -- & 62.4 \\
FCA-202-47 & 0.6 & 7.0 & -- & -- & -- & -- & 59.1 \\
FCA-201-47 & 1.0 & 0.7 & -- & -- & -- & - & 72.0 \\
FCA-188-47 & 0.4 & 5.7 & -- & -- & -- & -- & 62.5 \\
FCA-187-47 & 0.6 & 0.6 & -- & -- & -- & -- & 70.0 \\
\hline
\end{tabular}

136.50

\footnotetext{
${ }^{1}$ Fossil collection made by H. Wedow, Paleontology and Stratigraphy Branch, U. S. Geological Survey.
} 


\begin{tabular}{|c|c|c|c|c|c|c|c|c|c|c|c|}
\hline \multirow[b]{2}{*}{$\begin{array}{c}\text { Bed } \\
\text { no. }\end{array}$} & \multirow[b]{2}{*}{ Rock description } & \multirow[b]{2}{*}{$\begin{array}{c}\text { Sample } \\
\text { no. }\end{array}$} & \multirow{2}{*}{$\begin{array}{l}\text { Thickness } \\
\text { (feet) }\end{array}$} & \multicolumn{6}{|c|}{ Chemical analyses (percent) } & \multirow{2}{*}{$\begin{array}{c}\text { Cumulative } \\
\text { thickness } \\
\text { (feet) }\end{array}$} & \multirow{2}{*}{$\begin{array}{l}\text { Thickness } x \\
\text { percent } \mathrm{P}_{2} \mathrm{O}_{5} \\
\text { (cumulative) }\end{array}$} \\
\hline & & & & $\mathrm{P}_{2} \mathrm{O}_{5}$ & $\mathrm{Al}_{2} \mathrm{O}_{3}$ & $\mathrm{Fe}_{2} \mathrm{O}_{3}$ & $F$ & $\begin{array}{l}\text { Loss on } \\
\text { ignition }\end{array}$ & $\begin{array}{c}\text { Acid } \\
\text { insoluble }\end{array}$ & & \\
\hline $\begin{array}{l}P-151 \\
P-150 \\
P-149 \\
P-148 \\
P-147\end{array}$ & $\begin{array}{l}\text { Limestone, argillaceous; fos. } \\
\text { col. no. 47-HW-70 } \\
\text { Mudstone, calcareous } \\
\text { Phosphate rock } \\
\text { Phosphate rock } \\
\text { Phosphate rock }\end{array}$ & $\begin{array}{l}\text { FCA-200-47 } \\
\text { FCA-199-47 } \\
\text { FCA-198-47 } \\
\text { FCA-197-47 } \\
\text { FCA-196-47 }\end{array}$ & $\begin{array}{l}1.4 \\
0.65 \\
0.8 \\
0.75 \\
0.5\end{array}$ & $\begin{array}{r}0.7 \\
3.4 \\
31.3 \\
33.1 \\
30.9\end{array}$ & $\begin{array}{l}-- \\
-- \\
0.49 \\
0.8 \\
1.1\end{array}$ & $\begin{array}{l}-- \\
-\overline{-} \\
0.43 \\
0.34 \\
04.3\end{array}$ & $\begin{array}{l}- \\
-- \\
3.30 \\
3.54 \\
3.26\end{array}$ & $\begin{array}{l}-- \\
9.42 \\
6.48 \\
7.82\end{array}$ & $\begin{array}{r}45.6 \\
64.2 \\
3.7 \\
5.7 \\
7.9\end{array}$ & $\begin{array}{l}18.7 \\
19.35 \\
20.15 \\
20.90 \\
21.40\end{array}$ & $\begin{array}{r}67.99 \\
70.20 \\
95.24 \\
120.04 \\
135.49\end{array}$ \\
\hline $\begin{array}{l}P-146 \\
P-145 \\
P-144 \\
P-143 \\
P-142\end{array}$ & $\begin{array}{l}\text { Limestone, phosphatic } \\
\text { Phosphate rock } \\
\text { Phosphate rock, argillaceous } \\
\text { Mudstone, calcareous } \\
\text { Phosphate rock, argillaceous }\end{array}$ & $\begin{array}{l}\text { FCA }-195-47 \\
\text { FCA } 194-47 \\
\text { FCA-193-47 } \\
\text { FCA } 192-47 \\
\text { FCA } 191-47\end{array}$ & $\begin{array}{l}0.5 \\
1.2 \\
0.18 \\
0.55 \\
0.35\end{array}$ & $\begin{array}{r}12.1 \\
33.3 \\
22.9 \\
2.4 \\
18.9\end{array}$ & $\begin{array}{l}0.3 \\
0.3 \\
-- \\
--\end{array}$ & $\begin{array}{c}0.08 \\
0.25 \\
-- \\
--\end{array}$ & $\begin{array}{l}-- \\
-- \\
-- \\
--\end{array}$ & $\begin{array}{c}29.16 \\
6.38 \\
-- \\
-- \\
--\end{array}$ & $\begin{array}{r}3.8 \\
4.8 \\
29.3 \\
47.1 \\
30.9\end{array}$ & $\begin{array}{l}21.90 \\
23.10 \\
23.28 \\
23.83 \\
24.18\end{array}$ & $\begin{array}{l}141.54 \\
181.50 \\
185.62 \\
186.94 \\
193.55\end{array}$ \\
\hline$P-141$ & $\begin{array}{l}\text { Limestone, argillaceous; fos. } \\
\text { col. no. } 47-\mathrm{HW}-181\end{array}$ & FCA-190-47 & 0.8 & 2.6 & -- & $\therefore$ & -- & -- & 35.8 & 24.98 & 195.63 \\
\hline $\begin{array}{l}P-140 \\
P-139\end{array}$ & $\begin{array}{l}\text { Mudstone, phosphatic } \\
\text { Limestone, argslaceous; }\end{array}$ & FCA $-189-47$ & 0.4 & 8.7 & -- & -- & -- & $=-$ & 55.9 & 25.38 & 199.61 \\
\hline $\begin{array}{l}P-138 \\
P-137\end{array}$ & $\begin{array}{l}\text { fos. col. no. 47-HW-180 } \\
\text { Phosphate rock } \\
\text { Mudstone, calcareous }\end{array}$ & $\begin{array}{l}\text { FCA-1 165-47 } \\
\text { FCA-164-47 } \\
\text { FCA-163-47 }\end{array}$ & $\begin{array}{l}1.1 \\
0.8 \\
0.65\end{array}$ & $\begin{array}{r}1.1 \\
27.2 \\
5.7\end{array}$ & $\begin{array}{l}--. \\
2.3 \\
6.1\end{array}$ & $\begin{array}{l}-- \\
1.18 \\
2.28\end{array}$ & $\begin{array}{l}-- \\
--\end{array}$ & $\begin{array}{r}-\overline{-5} \\
17.84\end{array}$ & $\begin{array}{l}35.2 \\
16.3 \\
42.8\end{array}$ & $\begin{array}{l}26.48 \\
27.28 \\
27.93\end{array}$ & $\begin{array}{l}200.82 \\
222.58 \\
226.28\end{array}$ \\
\hline $\begin{array}{l}P-136 \\
P-135 \\
P-134 \\
P-133 \\
P-132\end{array}$ & $\begin{array}{l}\text { Phosphate rock } \\
\text { Limestone } \\
\text { Phosphate rock } \\
\text { Phosphate rock, argillaceous } \\
\text { Phosphate rock }\end{array}$ & $\begin{array}{l}\text { FCA-162-47 } \\
\text { FCA-161-47 } \\
\text { FCA-160-47 } \\
\text { FCA-159-47 } \\
\text { FCA-1 } 13-47\end{array}$ & $\begin{array}{l}0.55 \\
0.65 \\
0.4 \\
0.3 \\
0.55\end{array}$ & $\begin{array}{r}34.2 \\
4.9 \\
35.7 \\
26.8 \\
33.8\end{array}$ & $\begin{array}{l}0.57 \\
1.4 \\
0.7 \\
3.7 \\
1.1\end{array}$ & $\begin{array}{l}0.39 \\
0.43 \\
0.41 \\
1.50 \\
0.86\end{array}$ & $\begin{array}{l}-- \\
-- \\
-- \\
--\end{array}$ & $\begin{array}{r}5.60 \\
32.96 \\
4.10 \\
4.34 \\
4.54\end{array}$ & $\begin{array}{r}4.7 \\
12.0 \\
3.9 \\
21.9 \\
6.7\end{array}$ & $\begin{array}{l}28.48 \\
29.13 \\
29.53 \\
29.83 \\
30.38\end{array}$ & $\begin{array}{l}245.08 \\
248.26 \\
262.54 \\
270.58 \\
291.96\end{array}$ \\
\hline $\begin{array}{l}P-131 \\
P-130 \\
P-129 \\
P-128 \\
P-127\end{array}$ & $\begin{array}{l}\text { Phosphate rock } \\
\text { Phosphate rock } \\
\text { Phosphate rock, argillaceous } \\
\text { Phosphate rock } \\
\text { Phosphate rock, calcareous, } \\
\text { argillaceous }\end{array}$ & $\begin{array}{l}\text { FCA-112-47 } \\
\text { FCA-147-47 } \\
\text { FCA-146-47 } \\
\text { FCA-145-47 } \\
\text { FCA }-49-47\end{array}$ & $\begin{array}{l}1.0 \\
1.0 \\
0.95 \\
0.28 \\
0.6\end{array}$ & $\begin{array}{l}36.4 \\
33.8 \\
17.4 \\
27.8 \\
15.0\end{array}$ & $\begin{array}{l}0.4 \\
0.8 \\
7.0 \\
2.4 \\
6.0\end{array}$ & $\begin{array}{l}0.30 \\
0.58 \\
2.3 \\
1.12 \\
1.7\end{array}$ & $\begin{array}{l}-- \\
-- \\
-- \\
1.41\end{array}$ & $\begin{array}{r}3.38 \\
4.84 \\
6.44 \\
9.44 \\
15.22\end{array}$ & $\begin{array}{r}3.3 \\
5.8 \\
40.3 \\
13.2 \\
29.0\end{array}$ & $\begin{array}{l}31.38 \\
32.38 \\
33.33 \\
33.61 \\
34.21\end{array}$ & $\begin{array}{l}328.36 \\
362.16 \\
378.81 \\
386.61 \\
395.61\end{array}$ \\
\hline $\begin{array}{l}P-126 \\
P-125 \\
P-124 \\
P-123 \\
P-122\end{array}$ & $\begin{array}{l}\text { Phosphate rock } \\
\text { Phosphate rock } \\
\text { Phosphate rock } \\
\text { Phosphate rock } \\
\text { Phosphate rock }\end{array}$ & $\begin{array}{l}\text { FCA- } 50-47 \\
\text { FCA- } 51-47 \\
\text { FCA- } 52-47 \\
\text { FCA- } 53-47 \\
\text { FCA- } 54-47\end{array}$ & $\begin{array}{l}0.4 \\
0.75 \\
0.5 \\
0.5 \\
0.4\end{array}$ & $\begin{array}{l}33.0 \\
28.0 \\
36.2 \\
36.2 \\
34.4\end{array}$ & $\begin{array}{l}1.8 \\
1.0 \\
0.9 \\
0.8 \\
1.4\end{array}$ & $\begin{array}{l}1.1 \\
0.55 \\
0.56 \\
0.48 \\
0.66\end{array}$ & $\begin{array}{l}-- \\
-- \\
-- \\
--\end{array}$ & $\begin{array}{l}5.40 \\
6.32 \\
4.96 \\
4.72 \\
5.92\end{array}$ & $\begin{array}{l}8.3 \\
8.6 \\
1.8 \\
1.9 \\
4.6\end{array}$ & $\begin{array}{l}34.61 \\
35.36 \\
35.86 \\
36.36 \\
36.76\end{array}$ & $\begin{array}{l}396.93 \\
417.93 \\
436.03 \\
454.13 \\
467.89\end{array}$ \\
\hline $\begin{array}{l}P-121 \\
P-120 \\
P-119 \\
P-118 \\
P-117\end{array}$ & $\begin{array}{l}\text { Phosphate rock } \\
\text { Phosphate rock } \\
\text { Phosphate rock } \\
\text { Phosphate rock } \\
\text { Phosphate rock }\end{array}$ & $\begin{array}{l}\text { FCA- } 64-47 \\
\text { FCA- } 65-47 \\
\text { FCA- } 66-47 \\
\text { FCA- } 67-47 \\
\text { FCA- } 68-47\end{array}$ & $\begin{array}{l}0.67 \\
0.35 \\
0.4 \\
0.6 \\
0.4\end{array}$ & $\begin{array}{l}35.2 \\
32.1 \\
25.9 \\
26.0 \\
28.6\end{array}$ & $\begin{array}{l}0.8 \\
1.8 \\
3.3 \\
2.2 \\
2.1\end{array}$ & $\begin{array}{l}0.34 \\
0.65 \\
1.2 \\
0.86 \\
0.84\end{array}$ & $\begin{array}{c}-- \\
-- \\
2.67 \\
--\end{array}$ & $\begin{array}{r}6.06 \\
7.44 \\
15.64 \\
11.04 \\
12.48\end{array}$ & $\begin{array}{r}3.4 \\
7.9 \\
14.9 \\
14.2 \\
10.2\end{array}$ & $\begin{array}{l}37.43 \\
37.78 \\
38.18 \\
38.78 \\
39.18\end{array}$ & $\begin{array}{l}489.39 \\
500.64 \\
511.00 \\
526.60 \\
538.04\end{array}$ \\
\hline $\begin{array}{l}P-116 \\
P-115 \\
P-114\end{array}$ & $\begin{array}{l}\text { Phosphate rock } \\
\text { Phosphate rock } \\
\text { Phosphate rock, argillaceous }\end{array}$ & $\begin{array}{l}\text { FCA- 69-47 } \\
\text { FCA-148-47 } \\
\text { FCA-149-47 }\end{array}$ & $\begin{array}{l}0.4 \\
0.8 \\
0.4\end{array}$ & $\begin{array}{l}32.8 \\
28.0 \\
24.1\end{array}$ & $\begin{array}{l}1.0 \\
2.3 \\
3.9\end{array}$ & $\begin{array}{l}0.55 \\
1.2 \\
1.3\end{array}$ & $\begin{array}{l}-- \\
--\end{array}$ & $\begin{array}{r}9.72 \\
12.34 \\
11.60\end{array}$ & $\begin{array}{r}4.9 \\
13.7 \\
22.3\end{array}$ & $\begin{array}{l}39.58 \\
40.38 \\
40.78\end{array}$ & $\begin{array}{l}551.16 \\
573.56 \\
583.20\end{array}$ \\
\hline
\end{tabular}




\begin{tabular}{|c|c|}
\hline$P-113$ & $\begin{array}{l}\text { Phosphate rock, calcareous, } \\
\text { argillaceous }\end{array}$ \\
\hline $\begin{array}{l}P-112 \\
P-111 \\
P-110 \\
P-109\end{array}$ & $\begin{array}{l}\text { Phosphate rock } \\
\text { Phosphate rock, argillaceous } \\
\text { Mudstone } \\
\text { Mudstone, phosphatic }\end{array}$ \\
\hline $\begin{array}{l}P-108 \\
P-107 \\
P-106 \\
P-105 \\
P-104\end{array}$ & $\begin{array}{l}\text { Mudstone, phosphatic } \\
\text { Mudstone } \\
\text { Mudstone } \\
\text { Mudstone } \\
\text { Phosphate rock; fos. col. no. } \\
\text { 47-HW-179 }\end{array}$ \\
\hline$P-103$ & $\begin{array}{l}\text { Mudstone, phosphatic, } \\
\text { calcareous }\end{array}$ \\
\hline$P-102$ & $\begin{array}{l}\text { Mudstone, phosphatic, } \\
\text { calcareous }\end{array}$ \\
\hline $\begin{array}{l}P-101 \\
P-100 \\
P-99\end{array}$ & $\begin{array}{l}\text { Mudstone, phosphatic } \\
\text { Phosphate rock, argillaceous } \\
\text { Mudstone }\end{array}$ \\
\hline $\begin{array}{l}P-98 \\
P=97\end{array}$ & $\begin{array}{l}\text { Mudstone, phosphatic } \\
\text { Mudstone; fos. col. no. } \\
47-\mathrm{HW}-178\end{array}$ \\
\hline P - & $\begin{array}{l}\text { Phosphate rock, argillaceous; } \\
\text { fos, col. no. } 47-\mathrm{HW}-177\end{array}$ \\
\hline P - & $\begin{array}{l}\text { Mudstone, phosphatic, } \\
\text { calcareous }\end{array}$ \\
\hline$P-94$ & $\begin{array}{l}\text { Mudstone, phosphatic, } \\
\text { calcareous }\end{array}$ \\
\hline $\begin{array}{l}P-93 \\
P=92 \\
P-91\end{array}$ & $\begin{array}{l}\text { Phosphate rock, calcareous } \\
\text { Phosphate rock, argillaceous } \\
\text { Phosphate rock and phosphatic } \\
\text { mudstone }\end{array}$ \\
\hline $\begin{array}{l}\text { P- } 90 \\
P-89\end{array}$ & $\begin{array}{l}\text { Mudstone, phosphatic } \\
\text { Phosphate rock, argillaceous }\end{array}$ \\
\hline $\begin{array}{l}P-88 \\
P-87 \\
P-86 \\
P-85 \\
P-84\end{array}$ & $\begin{array}{l}\text { Phosphate rock } \\
\text { Mudstone } \\
\text { Phosphate rock } \\
\text { Limestone, argillaceous } \\
\text { Phosphate rock }\end{array}$ \\
\hline & $\begin{array}{l}\text { Limestone concretion in } \\
\text { bed P-84 }\end{array}$ \\
\hline $\begin{array}{l}\text { P- } 83 \\
\text { P- } 82 \\
\text { P- } 81 \\
\text { P- } 80\end{array}$ & $\begin{array}{l}\text { Phosphate rock, argillaceous } \\
\text { Phosphate rock, argillaceous } \\
\text { Mudstone, calcareous } \\
\text { Phosphate rock }\end{array}$ \\
\hline
\end{tabular}

\begin{tabular}{|c|c|c|c|c|}
\hline $\begin{array}{l}\text { FCA-150-47 } \\
\text { FCA-151-47 } \\
\text { RAG- } 25-47 \\
\text { RAG- } 26-47 \\
\text { RAG- } 27-47\end{array}$ & $\begin{array}{l}0.35 \\
0.8 \\
2.6 \\
1.0 \\
0.56\end{array}$ & $\begin{array}{l}21.1 \\
27.4 \\
20.1 \\
1.9 \\
13.6\end{array}$ & $\begin{array}{l}5.4 \\
2.3 \\
5.1 \\
-- \\
--\end{array}$ & $\begin{array}{l}1.9 \\
0.78 \\
1.8 \\
-- \\
--\end{array}$ \\
\hline $\begin{array}{l}\text { RAG- } 28-47 \\
\text { RAG- } 29-47 \\
\text { RAG- } 30-47 \\
\text { RAG- } 31-47\end{array}$ & $\begin{array}{l}1.55 \\
0.87 \\
0.92 \\
0.88\end{array}$ & $\begin{array}{r}13.7 \\
2.8 \\
3.0 \\
2.2\end{array}$ & $\begin{array}{l}-- \\
-- \\
--\end{array}$ & $\begin{array}{l}-- \\
-- \\
--\end{array}$ \\
\hline RAG- $32-47$ & 0.55 & 29.4 & -- & -- \\
\hline RAG- $33-47$ & 0.87 & 14.2 & -- & -- \\
\hline $\begin{array}{l}\text { RAG- } 34-47 \\
\text { RAG- } 35-47 \\
\text { RAG- } 36-47 \\
\text { RAG- } 37-47\end{array}$ & $\begin{array}{l}0.55 \\
1.45 \\
0.42 \\
1.1\end{array}$ & $\begin{array}{r}11.4 \\
9.7 \\
18.5 \\
7.6\end{array}$ & $\begin{array}{l}-- \\
-- \\
--\end{array}$ & $\begin{array}{l}-- \\
-- \\
--\end{array}$ \\
\hline RAG- $38-47$ & 0.37 & 11.6 & -- & -- \\
\hline RAG- $39-47$ & 0.66 & 7.5 & -- & -- \\
\hline RAG- $40-47$ & 1.9 & 17.4 & -- & -- \\
\hline RAG- $41-47$ & 1.75 & 15.5 & -- & -- \\
\hline RAG- $42-47$ & 0.91 & 12.1 & -- & -- \\
\hline $\begin{array}{l}\text { RAG- } 43-47 \\
\text { RAG- } 44-47\end{array}$ & $\begin{array}{l}0.97 \\
0.58\end{array}$ & $\begin{array}{l}18.1 \\
16.4\end{array}$ & 8.2 & $2 . \overline{6}$ \\
\hline $\begin{array}{l}\text { RAG- } 45-47 \\
\text { RAG- } 46-47 \\
\text { RAG- } 47-47\end{array}$ & $\begin{array}{l}0.96 \\
0.48 \\
0.55\end{array}$ & $\begin{array}{r}31.6 \\
8.4 \\
23.7\end{array}$ & $\begin{array}{r}1.7 \\
11.6 \\
5.4\end{array}$ & $\begin{array}{l}0.68 \\
3.6 \\
1.8\end{array}$ \\
\hline $\begin{array}{l}\text { RAG- } 48-47 \\
\text { RAG- } 49-47 \\
\text { RAG- } 50-47 \\
\text { RAG- } 52-47 \\
\text { RAG- } 53-47\end{array}$ & $\begin{array}{l}1.75 \\
0.82 \\
0.55 \\
0.31 \\
2.53\end{array}$ & $\begin{array}{r}31.6 \\
7.0 \\
28.0 \\
6.8 \\
24.9\end{array}$ & $\begin{array}{r}1.8 \\
11.3 \\
3.0 \\
-- \\
--\end{array}$ & $\begin{array}{l}0.74 \\
3.6 \\
1.1 \\
-- \\
--\end{array}$ \\
\hline $\begin{array}{l}\text { RAG- } 54-47 \\
\text { RAG- } 55-47 \\
\text { RAG- } 56-47 \\
\text { RAG- } 57-47 \\
\text { RAG- } 58-47\end{array}$ & $\begin{array}{c}(0.0-1.2) \\
0.51 \\
1.0 \\
0.64 \\
2.2\end{array}$ & $\begin{array}{r}2.4 \\
20.5 \\
23.4 \\
3.5 \\
27.3\end{array}$ & $\begin{array}{l}-- \\
-- \\
-- \\
--\end{array}$ & $\begin{array}{l}-- \\
-- \\
-- \\
--\end{array}$ \\
\hline
\end{tabular}

\begin{tabular}{c}
15.00 \\
13.62 \\
15.16 \\
-- \\
- \\
-- \\
-- \\
-- \\
-- \\
-- \\
-- \\
-- \\
-- \\
-- \\
-- \\
-- \\
-- \\
-- \\
10.14 \\
8.80 \\
9.12 \\
10.56 \\
9.20 \\
9.20 \\
-- \\
-- \\
-- \\
-- \\
-- \\
-- \\
\hline-
\end{tabular}

41.13

41.93

44.53

45.53

46. 09

47. 64

48.51

49.43

50.31

50.86

51.73

52.28

53. 73

54.15

55.25

55.62

56.28

58.18

59.93

60.84

61.81

62.39

63.35

63.83

64.38

66.13

66.95

67.50

70.34

39.7
18.7

11.9

29.4

23.1

39.3
14.2

71.85

72.49

$\begin{array}{r}74.69 \\ \hline\end{array}$
590.60

612.52

664.78

666.68

674.28

695.48

697.92

700.68

702.62

718.82

731.20

737.58

751.68

759.43

767.79

772.08

777.03

810.09

837. 19

848. 19

865. 74

875.25

895.65

899.69

912.74

967.99

973.73

989.13

991.24

$1,054.24$

$70.85^{\circ}$ 


\begin{tabular}{|c|c|c|c|c|c|c|c|c|c|c|c|}
\hline \multirow[b]{2}{*}{$\begin{array}{c}\text { Bed } \\
\text { no. }\end{array}$} & \multirow[b]{2}{*}{ Rock description } & \multirow[b]{2}{*}{$\begin{array}{l}\text { Sample } \\
\text { no. }\end{array}$} & \multirow[b]{2}{*}{$\begin{array}{l}\text { Thickness } \\
\text { (feet) }\end{array}$} & \multicolumn{6}{|c|}{ Chemical analyses (percent) } & \multirow{2}{*}{$\begin{array}{c}\text { Cumulative } \\
\text { thickness } \\
\text { (feet) }\end{array}$} & \multirow{2}{*}{$\begin{array}{l}\text { Thickness } \mathrm{x} \\
\text { percent } \mathrm{P}_{2} \mathrm{O}_{5} \\
\text { (cumulative) }\end{array}$} \\
\hline & & & & $\mathrm{P}_{2} \mathrm{O}_{5}$ & $\mathrm{Al}_{2} \mathrm{O}_{3}$ & $\mathrm{Fe}_{2} \mathrm{O}_{3}$ & $F$ & $\begin{array}{l}\text { Loss on } \\
\text { ignition }\end{array}$ & $\begin{array}{c}\text { Acid } \\
\text { insoluble }\end{array}$ & & \\
\hline P- 79 & Limestone, argillaceous & RAG- $59-47$ & 1.55 & 4.8 & -- & -- & 0.51 & - & 20.5 & 76.24 & $1,158.49$ \\
\hline$P-78$ & $\begin{array}{l}\text { Phosphate rock, argillaceous, } \\
\text { calcareous }\end{array}$ & RAG- $60-47$ & 2.23 & 17.5 & -- & -- & -- & -- & 33.0 & 78.47 & $1,197.49$ \\
\hline$P-77$ & Mudstone, phosphatic & RAG- $61-47$ & 1.4 & 10.6 & -- & -- & -- & - & 56.6 & 79.87 & $1,212.33$ \\
\hline P- 76 & $\begin{array}{l}\text { Mudstone, phosphatic, } \\
\text { calcareous }\end{array}$ & RAG- $65-47$ & 1.6 & 14.0 & -- & -- & -- & -- & 39.3 & 81.47 & $1,234.73$ \\
\hline$P-75$ & $\begin{array}{l}\text { Mudstone, calcareous, } \\
\text { phosphatic }\end{array}$ & RAG- $66-47$ & 3.55 & 11.5 & -- & -- & $-\infty$ & -- & 37.8 & 85.02 & $1,275.63$ \\
\hline P- 74 & Limestone & RAG- $67-47$ & 1.17 & 1.5 & -- & - & -- & -- & 4.8 & 86.19 & $1,277.38$ \\
\hline$P=73$ & $\begin{array}{l}\text { Limestone, phosphatic, } \\
\text { argillaceous }\end{array}$ & RMC $-22-47$ & 0.7 & 13.4 & -- & $-\infty$ & -- & -- & 30.1 & 86.89 & $1,286.76$ \\
\hline$P-72$ & Phosphate rock, calcareous & RMC- $21-47$ & 1.7 & 19.3 & 4.1 & 1.3 & - & 18.92 & 15.2 & 88.59 & $1,319.57$ \\
\hline$P-71$ & Phosphate rock, calcareous & RMC $-20-47$ & 0.5 & 22.4 & 2.8 & 1.25 & - & 17.42 & 13.0 & 89.09 & $1,330.77$ \\
\hline$P-70$ & Phosphate rock, calcareous & RMC $-19-47$ & 1.1 & 22.7 & 4.1 & 1.5 & -- & 14.86 & 16.7 & 90.19 & $1,355.74$ \\
\hline & $\begin{array}{l}\text { Phosphate rock, argillaceous, } \\
\text { calcareous }\end{array}$ & RMC- $18-47$ & 0.3 & 22.2 & 4.3 & 1.83 & -- & 13.44 & 22.0 & 90.49 & $1,362.40$ \\
\hline$P-68$ & Phosphate rock & RAG- $76-47$ & 1.25 & 25.5 & 3.5 & 1.2 & - & 14.40 & 13.8 & 91.74 & $1,394.30$ \\
\hline $\begin{array}{l}P-67 \\
P-66 \\
P-65\end{array}$ & $\begin{array}{l}\text { Phosphate rock } \\
\text { Phosphate rock } \\
\text { Phosphate rock, calcareous }\end{array}$ & $\begin{array}{l}\text { RAG- } 75-47 \\
\text { RAG- } 74-47 \\
\text { RAG- } 73-47\end{array}$ & $\begin{array}{l}1.95 \\
1.45 \\
0.33\end{array}$ & $\begin{array}{l}32.2 \\
31.9 \\
19.1\end{array}$ & $\begin{array}{l}0.9 \\
1.0 \\
2.4\end{array}$ & $\begin{array}{l}0.36 \\
0.37 \\
1.07\end{array}$ & $\begin{array}{l}-- \\
--\end{array}$ & $\begin{array}{r}9.18 \\
10.44 \\
20.90\end{array}$ & $\begin{array}{r}3.5 \\
3.8 \\
12.5\end{array}$ & $\begin{array}{l}93.69 \\
95.14 \\
95.47\end{array}$ & $\begin{array}{l}1,457.10 \\
1,503.35 \\
1,509.71\end{array}$ \\
\hline P- 64 & Limestone & RAG- $72-47$ & 0.85 & 2.9 & 0.3 & 0.06 & - & 42.38 & 1.4 & 96.32 & $1,512.18$ \\
\hline- & $\begin{array}{l}\text { Limestone concretion in bed } \\
P-63 \text { and lower part of } \\
\text { bed } P-64\end{array}$ & RAG $-71-47$ & $(0.0-3.0)$ & 0.3 & 1.3 & 0.41 & -- & 41.98 & 8.6 & -- & - \\
\hline & $\begin{array}{l}\text { Phosphate rock, calcareous; } \\
\text { fos. col. no. } 47-\mathrm{HW}-176\end{array}$ & RAG- $70-47$ & 2.66 & 22.0 & 4.4 & 1.4 & -- & 16.40 & 16.7 & 98.98 & $1,570.68$ \\
\hline$P-62$ & $\begin{array}{l}\text { Mudstone, calcareous, } \\
\text { phosphatic }\end{array}$ & RAG- $69-47$ & 0.33 & 8.6 & -- & -- & - & -- & $4 \zeta .8$ & 99.31 & $1,573.52$ \\
\hline$P-61$ & Limestone & RAG- $68-47$ & 1.35 & 0.9 & -- & -- & -- & -- & 15.7 & 100.66 & $1,574.74$ \\
\hline$P-60$ & Limestone & RMC - 16-47 & 0.6 & 0.5 & - & -- & - & - & 14.2 & 101.26 & $1,575.04$ \\
\hline$P-59$ & Limestone & RMC - 15-47 & 0.9 & 0.7 & -- & -- & - & -- & 8.6 & 102.16 & $1,575.67$ \\
\hline$P-58$ & Limestone & RMC - 14-47 & 1.6 & 0.6 & -- & -- & -- & - & 9.3 & 103.76 & $1,576.63$ \\
\hline $\begin{array}{l}P-57 \\
P-56\end{array}$ & $\begin{array}{l}\text { Limestone, argillaceous } \\
\text { Limestone }\end{array}$ & $\begin{array}{l}\text { RMC }-13-47 \\
\text { RMC- } 12-47\end{array}$ & $\begin{array}{l}0.4 \\
0.6\end{array}$ & $\begin{array}{l}0.5 \\
0.4\end{array}$ & -- & -- & -- & $-\infty$ & $\begin{array}{l}44.2 \\
13.9\end{array}$ & $\begin{array}{l}104.16 \\
104.76\end{array}$ & $\begin{array}{l}1,576.83 \\
1,577.07\end{array}$ \\
\hline$P-55$ & Limestone & RMC - 11-47 & 1.8 & 0.4 & -- & -- & - & -- & 14.0 & 106.56 & $1,577.79$ \\
\hline $\mathrm{P}-54$ & Limestone, argillaceous & FCA-228-47 & 1.1 & 0.9 & -- & -- & - & -- & 27.4 & 107.66 & $1,578.78$ \\
\hline$P-53$ & Mudstone, calcareous & FCA $-227-47$ & 0.75 & 2.0 & -- & - & - & -- & 62.6 & 108.41 & $1,580.28$ \\
\hline & $\begin{array}{l}\text { Limestone concretion in } \\
\text { bed } P-53\end{array}$ & FCA $-226-47$ & $(0.0-1.2)$ & 0.2 & - & -- & - & - & 14.9 & -- & -- \\
\hline$P-52$ & Mudstone & FCA $-225-47$ & 0.9 & 2.7 & - & -- & & -- & 66.0 & 109.31 & $1,582.71$ \\
\hline $\begin{array}{l}P-51 \\
P-50\end{array}$ & $\begin{array}{l}\text { Mudstone } \\
\text { Mudstone }\end{array}$ & $\begin{array}{l}\text { FCA-224-47 } \\
\text { FCA-223-47 }\end{array}$ & $\begin{array}{l}1.0 \\
0.9\end{array}$ & $\begin{array}{l}4.0 \\
8.6\end{array}$ & -- & -- & -- & -- & $\begin{array}{l}62.2 \\
50.0\end{array}$ & $\begin{array}{l}110.31 \\
111.21\end{array}$ & $\begin{array}{l}1,586.71 \\
1,594.45\end{array}$ \\
\hline
\end{tabular}




\begin{tabular}{|c|c|}
\hline -- & $\begin{array}{l}\text { Limestone concretion in } \\
\text { bed } P-49\end{array}$ \\
\hline$P-49$ & $\begin{array}{l}\text { Mudstone, calcareous, } \\
\text { phosphatic }\end{array}$ \\
\hline-- & $\begin{array}{l}\text { Limestone concretion in } \\
\text { bed } P-48\end{array}$ \\
\hline$P-48$ & $\begin{array}{l}\text { Mudstone, phosphatic, } \\
\text { calcareous }\end{array}$ \\
\hline$P-47$ & $\begin{array}{l}\text { Limestone; fos. col. no. } \\
47-\mathrm{HW}-71\end{array}$ \\
\hline$P-46$ & $\begin{array}{l}\text { Phosphate rock, calcareous, } \\
\text { argillaceous }\end{array}$ \\
\hline - & $\begin{array}{l}\text { Limestone concretion in } \\
\text { bed } P-46\end{array}$ \\
\hline$P-45$ & $\begin{array}{l}\text { Limestone, argillaceous, } \\
\text { phosphatic }\end{array}$ \\
\hline$P-44$ & $\begin{array}{l}\text { Mudstone, calcareous, } \\
\text { phosphatic }\end{array}$ \\
\hline$P-43$ & $\begin{array}{l}\text { Phosphate rock, calcareous, } \\
\text { argillaceous }\end{array}$ \\
\hline$P-42$ & $\begin{array}{l}\text { Limestone, phosphatic, } \\
\text { argillaceous }\end{array}$ \\
\hline$P-41$ & $\begin{array}{l}\text { Phospbate rock and calcareous } \\
\text { mudstone }\end{array}$ \\
\hline$P-40$ & Limestone \\
\hline$P-39$ & Mudstone, phosphatic \\
\hline$P-38$ & Mudstone, phosphatic \\
\hline$P-37$ & Mudstone, phosphatic \\
\hline$P=36$ & Mudstone, phosphatic \\
\hline P- 35 & $\begin{array}{l}\text { Limestone, phosphatic, } \\
\text { argillaceous }\end{array}$ \\
\hline-- & $\begin{array}{l}\text { Limestone concretion in } \\
\text { bed } P-34\end{array}$ \\
\hline P- 34 & $\begin{array}{l}\text { Limestone, phosphatic, } \\
\text { argillaceous }\end{array}$ \\
\hline P- 33 & $\begin{array}{l}\text { Limestone, phosphatic, } \\
\text { argillaceous }\end{array}$ \\
\hline$P-32$ & $\begin{array}{l}\text { Limestone, phosphatic, } \\
\text { argillaceous }\end{array}$ \\
\hline P- 31 & $\begin{array}{l}\text { Mudstone, phosphatic, } \\
\text { calcareous }\end{array}$ \\
\hline$P-$ & Phosphate rock, calcareous \\
\hline P- & Phosphate rock, calcareous \\
\hline$P-28$ & Limestone, phosphatic \\
\hline$P-27$ & $\begin{array}{l}\text { Mudstone, calcareous, } \\
\text { phosphatic }\end{array}$ \\
\hline$P-26$ & Phosphate rock \\
\hline
\end{tabular}

\begin{tabular}{|c|c|c|c|c|c|c|c|c|c|}
\hline FCA $-222-47$ & $(0.0-0.35)$ & 0.9 & -- & -- & -- & -- & 4.0 & -- & -- \\
\hline FCA $-221-47$ & 1.2 & 7.8 & -- & - & -- & -- & 38.7 & 112.41 & $1,603.81$ \\
\hline FCA $-220-47$ & $(0.0-0.45)$ & 0.8 & -- & -- & -- & -- & 2.6 & -- & -- \\
\hline FCA-219-47 & 1.4 & 8.8 & -- & $-\rightarrow$ & -- & -- & 51.3 & 113.81 & $1,616.13$ \\
\hline FCA $-218-47$ & 1.4 & 2.4 & -- & -- & -- & -- & 11.4 & 115.21 & $1,619.09$ \\
\hline FCA $-158-47$ & 1.3 & 15.6 & -- & -- & -- & -- & 25.7 & 116.51 & $1,639.37$ \\
\hline FCA-157-47 & $(0.0-1.0)$ & 2.3 & -- & -- & -- & -- & 8.7 & -- & -- \\
\hline FCA-156-47 & 0.9 & 9.6 & -- & -- & -- & -- & 32.5 & 117.41 & $1,648.06$ \\
\hline FCA-155-47 & 1.4 & 9.7 & -- & -- & -- & -- & 38.9 & 118.81 & $1,661.64$ \\
\hline FCA $-154-47$ & 0.8 & 15.1 & -- & -- & -- & -- & 29.1 & 119.61 & $1,673.72$ \\
\hline FCA-153-47 & 0.5 & 12.6 & -- & -- & -- & -- & 23.2 & 120.11 & $1,680,02$ \\
\hline $\begin{array}{l}\text { FCA-152-47 } \\
\text { FCA- 63-47 } \\
\text { FCA- 62-47 } \\
\text { FCA- } 61-47\end{array}$ & $\begin{array}{l}0.7 \\
0.95 \\
0.85 \\
0.6\end{array}$ & $\begin{array}{l}15.1 \\
1.3 \\
10.1 \\
11.0\end{array}$ & $\begin{array}{l}-- \\
-- \\
--\end{array}$ & $\begin{array}{l}=- \\
=- \\
--\end{array}$ & $\begin{array}{l}-- \\
-- \\
--\end{array}$ & $\begin{array}{l}-- \\
-- \\
--\end{array}$ & $\begin{array}{r}29.7 \\
3.2 \\
45.5 \\
39.5\end{array}$ & $\begin{array}{l}120.81 \\
121.76 \\
122.61 \\
123.21\end{array}$ & $\begin{array}{l}1,690.59 \\
1,691.83 \\
1,700.43 \\
1,707.03\end{array}$ \\
\hline $\begin{array}{l}\text { FCA- } 60-47 \\
\text { FCA- } 59-47\end{array}$ & $\begin{array}{l}0.45 \\
0.75\end{array}$ & $\begin{array}{l}10.5 \\
12.2\end{array}$ & -- & $=$ & $=$ & $=$ & $\begin{array}{l}44.6 \\
32.0\end{array}$ & $\begin{array}{l}123.66 \\
124.41\end{array}$ & $\begin{array}{l}1,711.73 \\
1,720.88\end{array}$ \\
\hline FCA $-58-47$ & 0.6 & 13.2 & -- & -- & -- & -- & 27.3 & 125.01 & $1,728.80$ \\
\hline FCA- 57-47 & $(0.0-0.8)$ & 1.4 & -- & -- & -- & -- & 2.7 & -- & -- \\
\hline FCA $-56-47$ & 0.9 & 11.0 & -- & - & -- & -- & 25.2 & 125.91 & $1,738.70$ \\
\hline FCA $-55-47$ & 1.0 & 13.3 & -- & -- & -- & -- & 28.1 & 126.91 & $1,752.00$ \\
\hline FCA-186-47 & 0.35 & 12.9 & -- & -- & $-\infty$ & -- & 30.3 & 127.26 & $1,756.51$ \\
\hline $\begin{array}{l}\text { FCA-185-47 } \\
\text { FCA-184-47 } \\
\text { FCA }-183-47\end{array}$ & $\begin{array}{l}0.65 \\
1.0 \\
0.8\end{array}$ & $\begin{array}{l}11.9 \\
17.4 \\
17.3\end{array}$ & $\begin{array}{l}-- \\
--\end{array}$ & $\begin{array}{l}-- \\
--\end{array}$ & $\begin{array}{l}-- \\
-- \\
--\end{array}$ & $\begin{array}{l}-- \\
-- \\
--\end{array}$ & $\begin{array}{l}27.9 \\
16.1 \\
11.7\end{array}$ & $\begin{array}{l}127.91 \\
128.91 \\
129.71\end{array}$ & $\begin{array}{l}1,764.25 \\
1,781.65 \\
1,795.49\end{array}$ \\
\hline FCA-182-47 & 0.95 & 16.3 & -- & -- & -- & -- & 13.3 & 130.66 & $1,810.99$ \\
\hline $\begin{array}{l}\text { FCA-181-47 } \\
\text { FCA } 180-47\end{array}$ & $\begin{array}{l}0.65 \\
0.6\end{array}$ & $\begin{array}{l}11.0 \\
24.2\end{array}$ & $=$ & -- & -- & $\overline{--}$ & $\begin{array}{l}32.7 \\
17.4\end{array}$ & $\begin{array}{l}131.31 \\
131.91\end{array}$ & $\begin{array}{l}1,818.14 \\
1,832.66\end{array}$ \\
\hline
\end{tabular}




\begin{tabular}{|c|c|c|c|c|c|c|c|c|c|c|c|}
\hline \multirow[b]{2}{*}{$\begin{array}{l}\text { Bed } \\
\text { no. }\end{array}$} & \multirow[b]{2}{*}{ Rock description } & \multirow{2}{*}{$\begin{array}{l}\text { Sample } \\
\text { no. }\end{array}$} & \multirow{2}{*}{$\begin{array}{l}\text { Thickness } \\
\text { (feet) }\end{array}$} & \multicolumn{6}{|c|}{ Chemical analyses (percent) } & \multirow{2}{*}{$\begin{array}{c}\text { Cumulative } \\
\text { thickness } \\
\text { (feet) }\end{array}$} & \multirow{2}{*}{$\begin{array}{l}\text { Thickness } \mathbf{x} \\
\text { percent } \mathbf{P}_{2} \mathbf{O} \\
\text { (cumulative) }\end{array}$} \\
\hline & & & & $\mathrm{P}_{2} \mathrm{O}_{5}$ & $\mathrm{Al}_{2} \mathrm{O}_{3}$ & $\mathrm{Fe}_{2} \mathrm{O}_{3}$ & $\mathbf{F}$ & $\begin{array}{l}\text { Loss on } \\
\text { ignition }\end{array}$ & $\begin{array}{l}\text { Acid } \\
\text { insoluble }\end{array}$ & & \\
\hline $\begin{array}{l}P-25 \\
P-24 \\
P-23 \\
P=22 \\
P-21\end{array}$ & $\begin{array}{l}\text { Limestone } \\
\text { Phosphate rock, argillaceous } \\
\text { Limestone, argillaceous } \\
\text { Mudstone, phosphatic } \\
\text { Mudstone, calcareous }\end{array}$ & $\begin{array}{l}\text { FCA-179-47 } \\
\text { FCA-178-47 } \\
\text { FCA-177-47 } \\
\text { FCA-1 } 72-47 \\
\text { FCA-171-47 }\end{array}$ & $\begin{array}{l}0.35 \\
0.45 \\
1.7 \\
2.6 \\
0.8\end{array}$ & $\begin{array}{r}3.4 \\
14.7 \\
3.8 \\
9.6 \\
6.8\end{array}$ & $\begin{array}{l}-- \\
-- \\
-- \\
-\infty\end{array}$ & $\begin{array}{l}-- \\
-- \\
-- \\
--\end{array}$ & $\begin{array}{l}-- \\
-- \\
\overline{--} \\
0.63\end{array}$ & $\begin{array}{l}-- \\
-- \\
-- \\
--\end{array}$ & $\begin{array}{l}10.2 \\
30.9 \\
17.5 \\
49.1 \\
58.5\end{array}$ & $\begin{array}{l}132.26 \\
132.71 \\
134.41 \\
137.01 \\
137.81\end{array}$ & $\begin{array}{l}1,833.85 \\
1,840.46 \\
1,846.92 \\
1,871.88 \\
1,877.32\end{array}$ \\
\hline \multirow[t]{2}{*}{ P- 20} & Mudstone, phosphatic & FCA-170-47 & 1.0 & 8. 7 & -- & -- & -- & -- & 46.5 & 138.81 & $1,886.02$ \\
\hline & $\begin{array}{l}\text { At the north trench (lot no. 1207) } \\
\text { The strata at the south trenches } \\
\text { between the trenches was based } \\
\text { Lot no. } 1236\end{array}$ & $\begin{array}{l}\text { e strata at the } \\
\text { t no. } 1236 \text { ) we } \\
\text { a nodular zon }\end{array}$ & $\begin{array}{l}\text { ase of the } \\
\text { measure }\end{array}$ & hospl & $\begin{array}{l}c \text { sh } \\
\text { pled }\end{array}$ & are & au & $\begin{array}{l}\text { tact } \\
\text { orma }\end{array}$ & $\begin{array}{l}\text { the und } \\
\text { on this }\end{array}$ & $\begin{array}{l}\text { W Wells fo } \\
\text { al. Corr }\end{array}$ & on. \\
\hline $\begin{array}{l}P-19 \\
P-18 \\
P-17 \\
P-16 \\
--\end{array}$ & $\begin{array}{l}\text { Limestone, argillaceous } \\
\text { Phosphate rock and mudstone } \\
\text { Phosphate rock and mudstone } \\
\text { Limestone, argillaceous } \\
\text { Limestone lens in bed P-15 }\end{array}$ & $\begin{array}{l}3053-\text { RPS } \\
3052-\text { RPS } \\
\text { 3051-RPS } \\
2859-\text { RPS } \\
\end{array}$ & $\begin{array}{l}2.7 \\
3.2 \\
1.1 \\
0.75 \\
(0.0-1.2)\end{array}$ & $\begin{array}{r}2.8 \\
23.6 \\
24.3 \\
7.6 \\
--\end{array}$ & $\begin{array}{l}-- \\
3.1 \\
3.7 \\
2.9 \\
-.\end{array}$ & $\begin{array}{l}1 .- \\
1.20 \\
1.35 \\
1.04 \\
--\end{array}$ & $\begin{array}{l}-- \\
-- \\
-- \\
--\end{array}$ & $\begin{array}{r}10.18 \\
8.30 \\
25.42 \\
--\end{array}$ & $\begin{array}{l}24.7 \\
26.0 \\
25.7 \\
26.7 \\
--\end{array}$ & $\begin{array}{l}141.51 \\
144.71 \\
145.81 \\
146.56\end{array}$ & $\begin{array}{l}1,893.58 \\
1,969.10 \\
1,995.83 \\
2,001.53 \\
\ldots-\end{array}$ \\
\hline $\begin{array}{l}P-15 \\
P-14 \\
P-13\end{array}$ & $\begin{array}{l}\text { Phosphate rock and mudstone } \\
\text { Phosphate rock } \\
\text { Phosphate rock argillaceous, }\end{array}$ & $\begin{array}{l}2858-\text { RPS } \\
2857-\text { RPS }\end{array}$ & $\begin{array}{l}3.6 \\
2.2\end{array}$ & $\begin{array}{l}20.8 \\
27.7\end{array}$ & $\begin{array}{l}4.1 \\
2.3\end{array}$ & $\begin{array}{l}1.46 \\
0.95\end{array}$ & -- & $\begin{array}{l}9.08 \\
9.12\end{array}$ & $\begin{array}{l}32.4 \\
16.6\end{array}$ & $\begin{array}{l}150.16 \\
152.36\end{array}$ & $\begin{array}{l}2,076.41 \\
2,137.35\end{array}$ \\
\hline $\begin{array}{l}P=13 \\
P=12 \\
P=11\end{array}$ & $\begin{array}{l}\text { Phosphate rock, argillaceous, } \\
\text { calcareous } \\
\text { Phosphate rock, argillaceous } \\
\text { Limestone }\end{array}$ & $\begin{array}{l}2856-\text { RPS } \\
2855-\text { RPS } \\
2854-\text { RPS }\end{array}$ & $\begin{array}{l}2.4 \\
2.1 \\
1.7\end{array}$ & $\begin{array}{r}17.2 \\
24.2 \\
2.4\end{array}$ & $\begin{array}{l}3.4 \\
3.5 \\
1.8\end{array}$ & $\begin{array}{l}1.26 \\
1.20 \\
0.41\end{array}$ & $\begin{array}{l}-- \\
--\end{array}$ & $\begin{array}{r}14.20 \\
8.68 \\
37.22\end{array}$ & $\begin{array}{l}29.5 \\
24.9 \\
11.4\end{array}$ & $\begin{array}{l}154.76 \\
156.86 \\
158.65\end{array}$ & $\begin{array}{l}2,178.63 \\
2,249.45 \\
2,233.53\end{array}$ \\
\hline P- 10 & $\begin{array}{l}\text { Phosphate rock, argillaceous, } \\
\text { calcareous }\end{array}$ & 2853-RPS & 1.3 & 20.0 & 3.2 & 1.03 & -- & 10.94 & 27.4 & 159.86 & $2,259.53$ \\
\hline & $\begin{array}{l}\text { Phosphate rock, argillaceous, } \\
\text { calcareous }\end{array}$ & 2852-RPS & 0.8 & 18.8 & -- & -- & - & -- & 27.4 & 160.66 & $2,274.57$ \\
\hline P- 8 & $\begin{array}{l}\text { Phosphate rock, calcareous, } \\
\text { argillaceous } \\
\text { imestone lens, argillaceous in }\end{array}$ & 2851-RPS & 0.7 & 17.3 & -- & -- & -- & - & 28.1 & 161.36 & $2,286.68$ \\
\hline$P=7$ & $\begin{array}{l}\text { Limestone lens, argillaceous in } \\
\text { bed P- } 7 \\
\text { Limestone }\end{array}$ & $\begin{array}{l}2850-\text { RPS } \\
1106-\text { RAG }\end{array}$ & $\begin{array}{c}(0.0-1.5) \\
1.8\end{array}$ & $\overline{5.1}$ & -- & -- & -- & -- & $\overline{8.4}$ & 163.16 & $2,29 \overline{5.86}$ \\
\hline $\begin{array}{l}\text { P- } 6 \\
P-\quad 5 \\
P-\quad 4 \\
P-\quad 3 \\
P-\quad 2\end{array}$ & $\begin{array}{l}\text { Phosphate rock } \\
\text { Phosphate rock } \\
\text { Mudstone, calcareous } \\
\text { Limestone, argillaceous } \\
\text { Mudstone, calcareous }\end{array}$ & $\begin{array}{l}1105-\mathrm{RAG} \\
1104-\mathrm{RAG} \\
1103-\mathrm{RAG} \\
1102-\mathrm{RAG} \\
1101-\mathrm{RAG}\end{array}$ & $\begin{array}{l}3.4 \\
2.4 \\
0.6 \\
2.6 \\
1.2\end{array}$ & $\begin{array}{r}31.4 \\
32.6 \\
2.7 \\
4.2 \\
3.6\end{array}$ & $\begin{array}{l}1.1 \\
0.58 \\
-- \\
-- \\
--\end{array}$ & $\begin{array}{c}0.51 \\
0.48 \\
-- \\
-- \\
--\end{array}$ & $\begin{array}{l}3.41 \\
3.57 \\
-- \\
-- \\
--\end{array}$ & $\begin{array}{l}8.54 \\
7.70 \\
-- \\
-- \\
--\end{array}$ & $\begin{array}{r}5.6 \\
2.6 \\
52.8 \\
33.5 \\
40.9\end{array}$ & $\begin{array}{l}166.56 \\
168.96 \\
169.56 \\
172.16 \\
173.36\end{array}$ & $\begin{array}{l}2,402.62 \\
2,480.86 \\
2,482.48 \\
2,493.40 \\
2,497.72\end{array}$ \\
\hline P- 1 & $\begin{array}{l}\text { Phosphate rock } \\
\text { Lot no. } 1207\end{array}$ & 1100-RAG & 0.4 & 33.6 & -- & -- & 3.57 & -- & 6.1 & 173.76 & $2,511.16$ \\
\hline$P-12$ & $\begin{array}{l}\text { Limestone, argillaceous; fos. col. } \\
\text { no. 47-HW-64 }\end{array}$ & FCA-169-47 & $(2.2)$ & 1.2 & -- & $-\infty$ & -- & -- & 22.0 & -- & -- \\
\hline
\end{tabular}




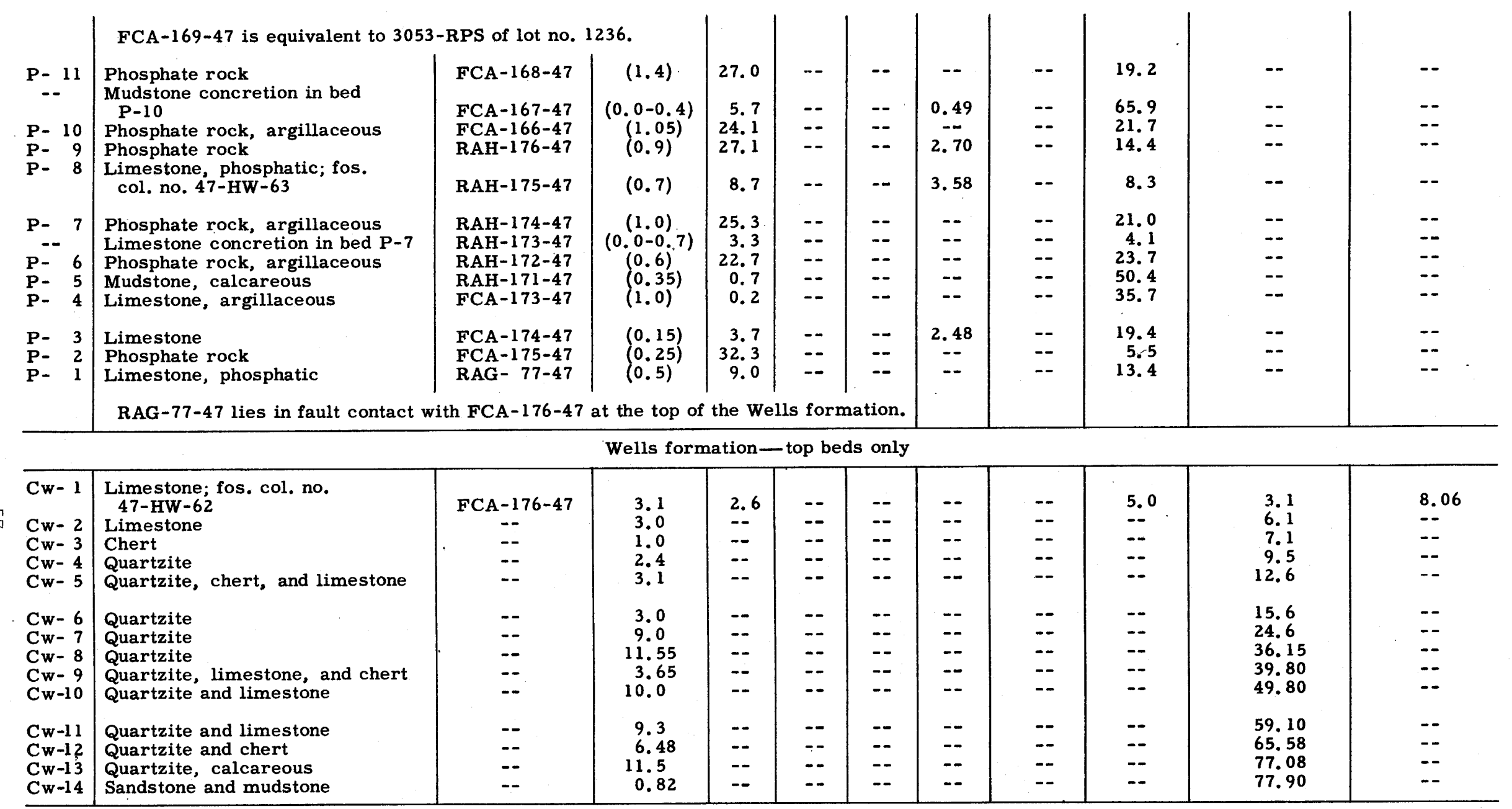


SPECTROGRAPHIC ANALYSES-MONTPELIER CANYON, IDAHO. LOT NO. 1207.

Semi-quantitative analyses of two samples of phosphatic shale member of Phosphoria formation, Montpelier Canyon, Idaho (see immediately preceding pages for location of section, thickness and description of strata, and chemical analyses of samples) made by $\mathrm{U}$. S. Bureau of Mines Laboratory, Albany, Oregon. In addition to the elements listed in the table below, Sb, $\mathrm{As}, \mathrm{Be}, \mathrm{Bi}, \mathrm{Cd}, \mathrm{Co}, \mathrm{Cb}$, $\mathrm{Ga}, \mathrm{Au}_{2} \mathrm{Li}, \mathrm{Hg}, \mathrm{Pt}, \mathrm{Ta}, \mathrm{Sn}, \mathrm{W}$, and $\mathrm{Zn}$ were looked for but were not detected.

Explanation of symbols
$A=$ more than 10 percent
$E=0.01-0.1$ percent
$B=5-10$ percent
$\mathrm{C}=1-5$ percent
$\mathrm{D}=0.1-1$ percent
$F=0.001-0.01$ percent
$G=$ less than 0.001 percent
$\mathrm{ND}=$ not detected

\begin{tabular}{|c|c|c|c|c|c|c|c|c|c|c|c|c|c|c|c|c|c|c|c|c|}
\hline $\begin{array}{r}\text { Bed } \\
\text { no. }\end{array}$ & Sample no. & Al & $\mathbf{B a}$ & B & $\mathrm{Ca}$ & $\mathrm{Cr}$ & $\mathrm{Cu}$ & $\mathrm{Fe}$ & $\mathrm{Pb}$ & $\mathbf{M g}$ & Mn & Mo & $\mathrm{Ni}$ & $\mathrm{Si}$ & Ag & $\mathrm{Na}$ & $\mathrm{Sr}$ & $\mathrm{Ti}$ & V & $\mathrm{Zr}$ \\
\hline $\begin{array}{l}P-132 \\
P-131\end{array}$ & $\begin{array}{l}\text { FCA-1 } 13-47 \\
\text { FCA-112-4 }\end{array}$ & $\begin{array}{l}\mathrm{D} \\
\mathrm{D}\end{array}$ & $\begin{array}{l}\mathbf{E} \\
\mathbf{E}\end{array}$ & $\begin{array}{l}\mathbf{F} \\
\mathbf{F}\end{array}$ & $\begin{array}{l}\mathrm{A} \\
\mathrm{A}\end{array}$ & $\begin{array}{l}\mathbf{E} \\
\mathbf{E}\end{array}$ & $\begin{array}{l}\mathbf{G} \\
\mathbf{G}\end{array}$ & $\begin{array}{l}\mathrm{D} \\
\mathrm{D}\end{array}$ & $\underset{\mathbf{N D}}{\mathrm{E}}$ & $\begin{array}{l}\mathrm{D} \\
\mathrm{D}\end{array}$ & $\begin{array}{l}\mathbf{F} \\
\mathbf{F}\end{array}$ & $\begin{array}{c}F \\
\text { ND }\end{array}$ & $\begin{array}{l}\mathbf{F} \\
\mathbf{F}\end{array}$ & $\begin{array}{l}\mathrm{C} \\
\mathrm{C}\end{array}$ & $\begin{array}{l}\mathbf{G} \\
\mathbf{G}\end{array}$ & $\begin{array}{l}\mathbf{E} \\
\mathbf{E}\end{array}$ & $\begin{array}{l}\mathbf{E} \\
\mathbf{E}\end{array}$ & $\begin{array}{l}\mathbf{E} \\
\mathbf{E}\end{array}$ & $\begin{array}{l}\mathrm{D} \\
\mathrm{D}\end{array}$ & $\begin{array}{l}F \\
F\end{array}$ \\
\hline
\end{tabular}




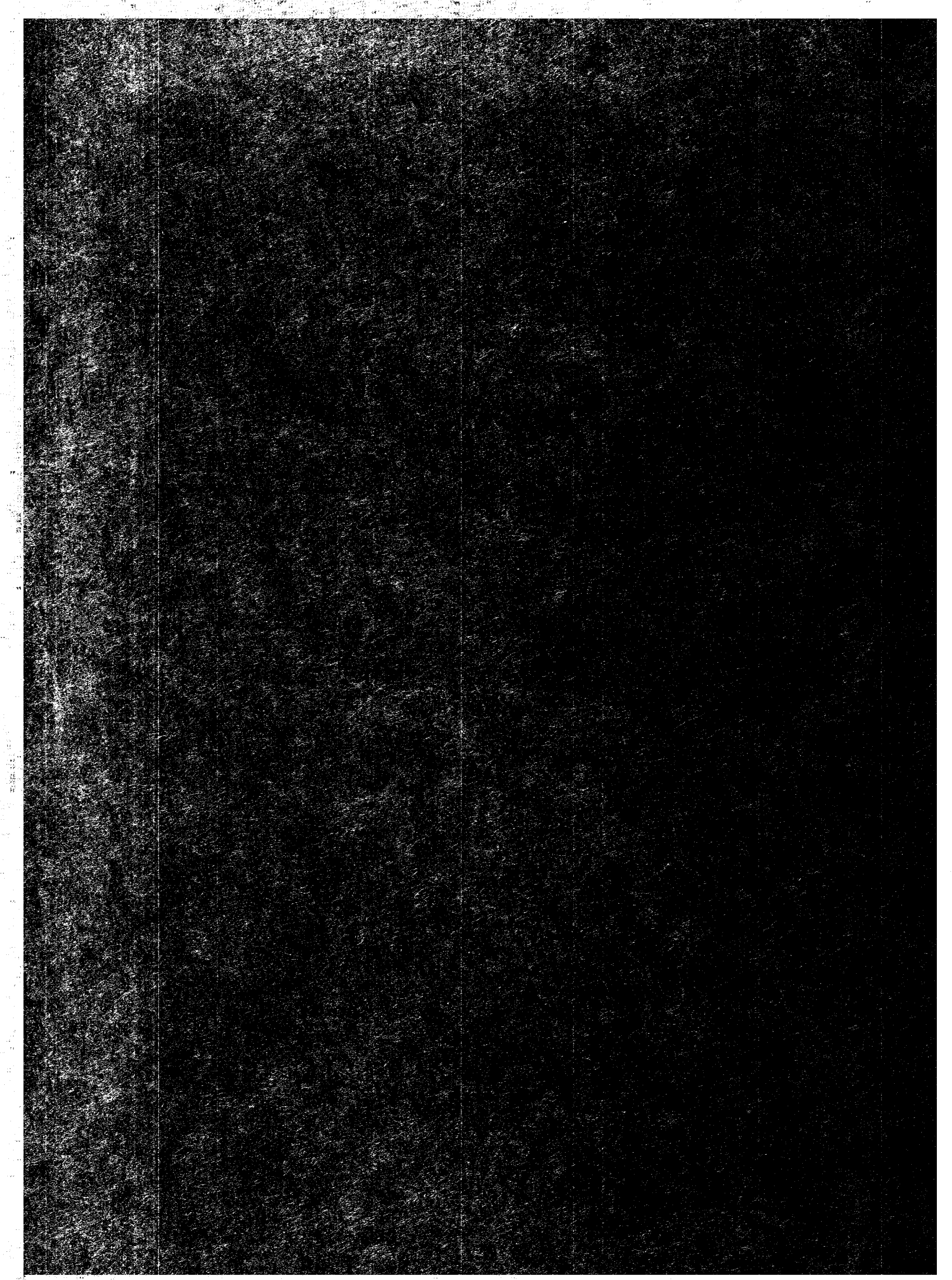

